University of Louisville

ThinkIR: The University of Louisville's Institutional Repository

Electronic Theses and Dissertations

$1-2018$

\title{
Development of a capacitive photocurrent scanning microscope with carrier depletion super-resolution.
}

\author{
Austin Levi Carver \\ University of Louisville
}

Follow this and additional works at: https://ir.library.louisville.edu/etd

Part of the Other Electrical and Computer Engineering Commons

\section{Recommended Citation}

Carver, Austin Levi, "Development of a capacitive photocurrent scanning microscope with carrier depletion super-resolution." (2018). Electronic Theses and Dissertations. Paper 3430.

https://doi.org/10.18297/etd/3430

This Doctoral Dissertation is brought to you for free and open access by ThinkIR: The University of Louisville's Institutional Repository. It has been accepted for inclusion in Electronic Theses and Dissertations by an authorized administrator of ThinkIR: The University of Louisville's Institutional Repository. This title appears here courtesy of the author, who has retained all other copyrights. For more information, please contact thinkir@louisville.edu. 
DEVELOPMENT OF A CAPACITIVE PHOTOCURRENT SCANNING MICROSCOPE WITH CARRIER DEPLETION SUPER-RESOLUTION

By

\author{
Austin Levi Carver \\ B.S., University of Louisville, 2012 \\ M.S., University of Louisville, 2013
}

\begin{abstract}
A Dissertation
Submitted to the Faculty of the

J.B. Speed School of Engineering at the University of Louisville in Partial Fulfillment of the Requirements for the Degree of
\end{abstract}

\author{
Doctor of Philosophy \\ in Electrical Engineering \\ Department of Electrical and Computer Engineering \\ University of Louisville \\ Louisville, Kentucky
}

May 2018 


\section{Copyright 2018 by Austin Levi Carver}

All rights reserved 

DEVELOPMENT OF A CAPACITIVE PHOTOCURRENT SCANNING MICROSCOPE WITH CARRIER DEPLETION SUPER-RESOLUTION

By

\author{
Austin Levi Carver \\ B.S., University of Louisville, 2012 \\ M.S., University of Louisville, 2013
}

A Dissertation Approved on

December 14, 2017

by the following Dissertation Committee:

Dr. Bruce W. Alphenaar

Dr. Sergio B. Mendes

Dr. Kevin M. Walsh

Dr. Cindy K. Harnett 


\section{ACKNOWLEDGMENTS}

This work is dedicated to my wife, Elizabeth, and my daughter, Eliana Mae, for their never-ending support and love. I must also thank my parents, Dr. D. Richard Carver, Jr. and Stephanie Carver, and my sister, Jessica Carver for their ever-present support and encouraging me to always ask questions. Finally, I am thankful for the support and encouragement of my grandparents, Richard and Dianna Carver.

I also want to express sincere thanks to my advisor, Dr. Bruce W. Alphenaar. Dr. Alphenaar taught me not only how to ask questions, but also what questions are the most important to ask. Without him I would not have come to develop a fuller understanding of both research and scientific inquiry. He was always there to answer my questions and guide my investigations. A special thanks to my co-advisor Dr. Sergio B. Mendes for opening up this $\mathrm{PhD}$ opportunity, as well as guiding me in my understanding of optics. I would also like to thank the other member of my committee, Dr. Cindy Harnett and Dr. Kevin Walsh for their guidance and advice along the way.

I would also like to thank Dr. Tommy Roussell, Dr. Hemant Shah, Dr. Rodrigo S. Wiederkehr, Jafar Ghithan, Sowmya Kolli, Kasun Fernando, Dr. Ji-Tzuoh Lin, and Pranoy Deb Shuvra for their help, discussions, and support throughout this journey. 


\begin{abstract}
DEVELOPMENT OF A CAPACITIVE PHOTOCURRENT SCANNING MICROSCOPE WITH CARRIER DEPLETION SUPER-RESOLUTION
\end{abstract}

\author{
Austin Levi Carver
}

December 14, 2017

This dissertation discusses the development and refinement of a new twodimensional imaging technique, funded in part through a NSF MRI equipment development grant. Capacitive-Photocurrent (CPC) spectroscopy allows for the probing of samples without the requirement of free-carrier collection. The CPC technique allows for the studying of various states within a material. With this electronic measurement technique, we developed a scanning technique, scanning-CPC, that provides twodimensional material property images without requiring environments that must be highvacuum, humidity-controlled, or temperature-controlled. This new technique also provides two-dimensional, electronic mapping without damaging samples. With this successful result, we then modified an existing resolution improving technique, Stimulated Emission Depletion (STED), to create a similar technique to improve the scanning-CPC resolution. The result was a new scanning technique, CPC-STED. With the CPC-STED technique we could achieve super-resolution of electronic response based images while maintaining the environmental flexibility of scanning-CPC. 
This dissertation is divided into six chapters, covering motivations and background, early work using the CPC technique, the development of the scanning-CPC system, testing and showcase of the scanning-CPC technique, testing and showcase of the CPC-STED technique, and finally, discussion of the results and proposals for future work. Chapter One discusses the motivations for this dissertation as well as the background of multiple one-dimensional and two-dimensional techniques. Chapter Two reviews the systems used for CPC spectroscopy, the previous work done in with the CPC technique, and the CPC work completed with my direct involvement. Chapter Three details the specifics of the scanning-CPC and CPC-STED system's optical layout, sample holder design, and the custom LabView design for the scanning system. Chapter Four details the results of the scanning-CPC system, focusing on the physical mechanisms leading to successful scanning-CPC images. Chapter Five details the results of the CPCSTED system. Chapter Five also discusses the specific outcomes of how the depletion technique functions in the context of electronic imaging, rather than fluorescence imaging. Finally, Chapter Six concludes the results and provides recommendations for future work. 
TABLE OF CONTENTS

PAGE

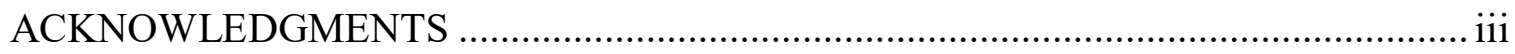

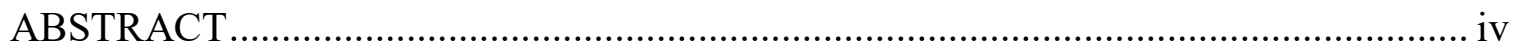

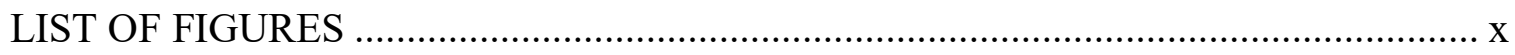

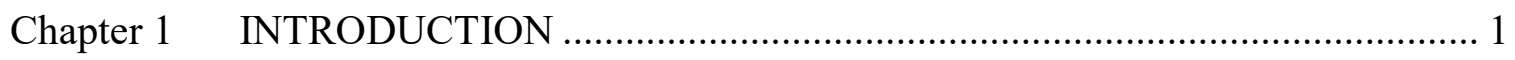

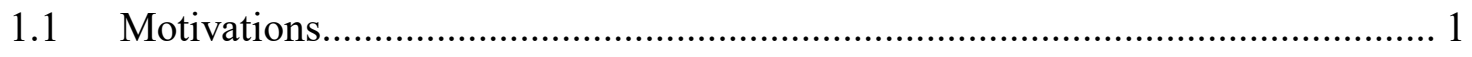

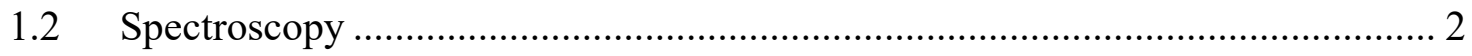

1.2.1 Absorbance spectroscopy .................................................................. 3

1.2.2 Photocurrent spectroscopy ................................................................. 5

1.2.3 Capacitive Photocurrent (CPC) spectroscopy .............................................. 6

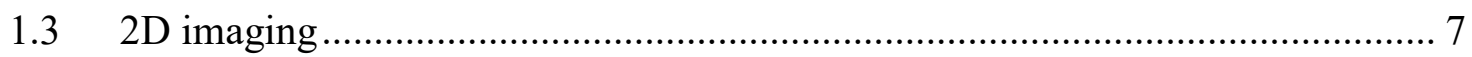

1.3.1 Atomic Force Microscopy (AFM) …………..................................... 8

1.3.2 Scanning Electron Microscope (SEM) ………....................................... 9

1.3.3 Electron Beam-Induced Current (EBIC) …………................................... 10

1.4 Sub-diffraction limited techniques .............................................................. 11

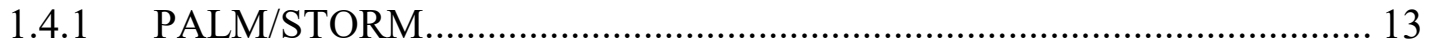

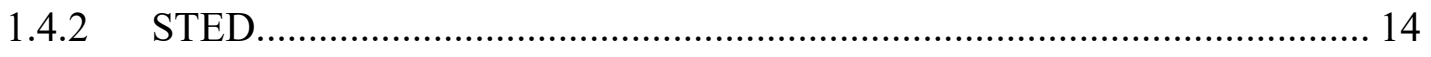


1.5 Combining CPC with STED. 18

\section{Chapter 2 FIXED-POINT CAPACITIVE PHOTOCURRENT TECHNIQUES AND}

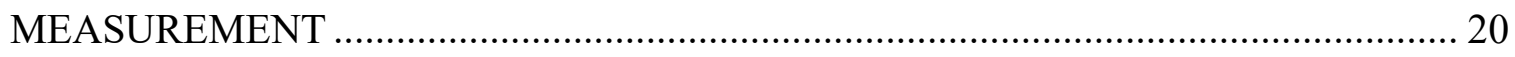

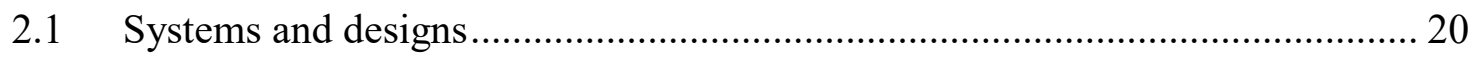

$2.1 .1 \quad$ OPA

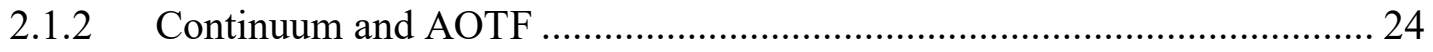

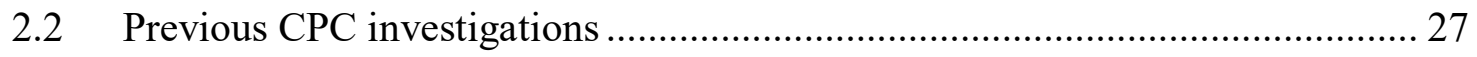

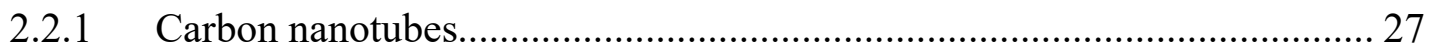

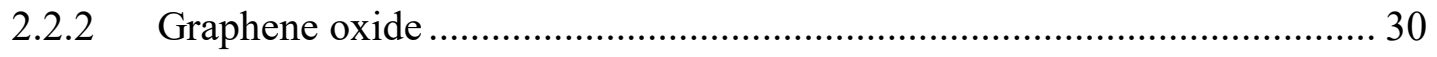

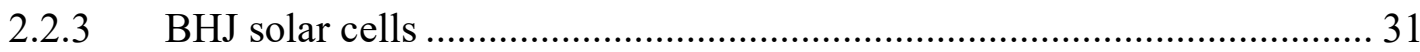

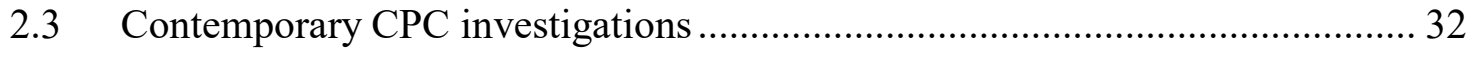

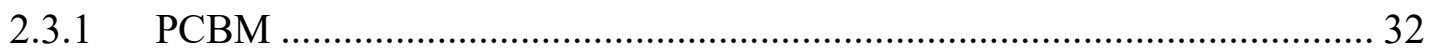

Chapter 3 DEVELOPMENT OF THE SCANNING-CPC TECHNIQUE................... 40

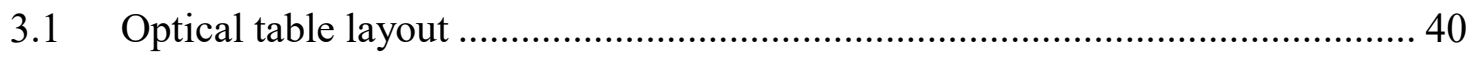

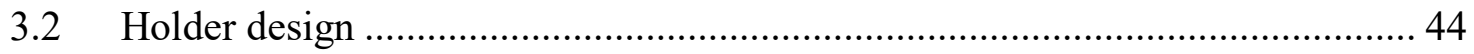

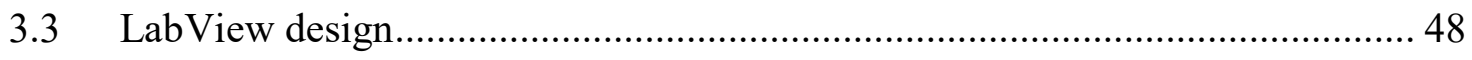

Chapter 4 RESULTS OF THE SCANNING-CPC SYSTEM ...................................... 56

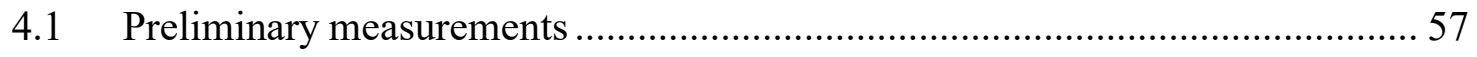

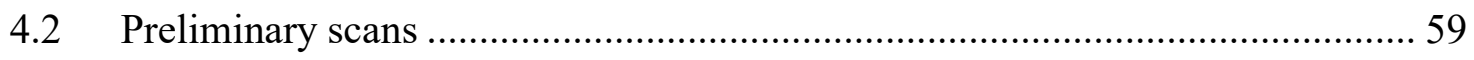

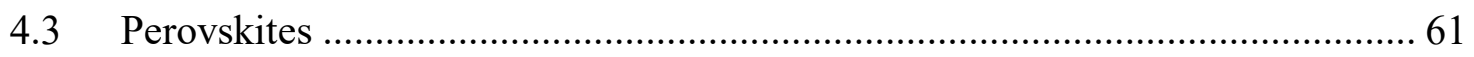


4.3.1 Preliminary work 62

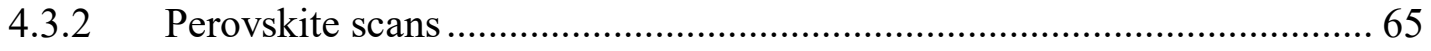

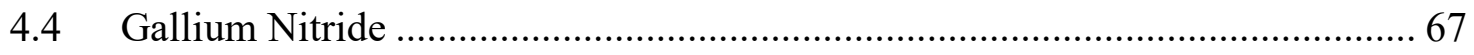

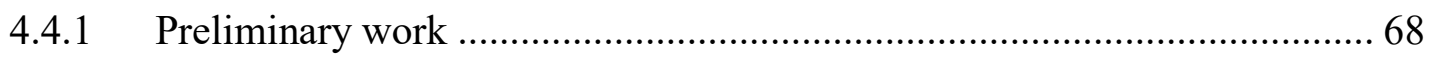

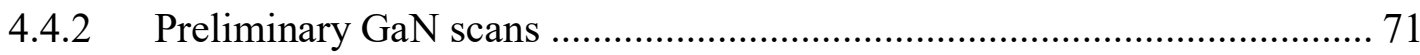

4.4.3 Testing electric field effects ............................................................ 74

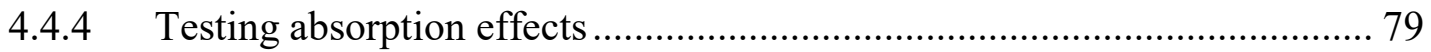

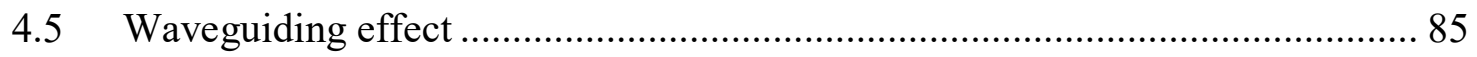

Chapter 5 CPC-STED SCANNING RESULTS .................................................. 91

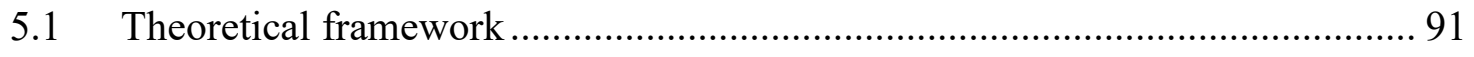

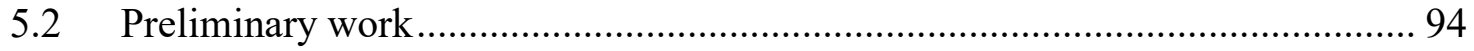

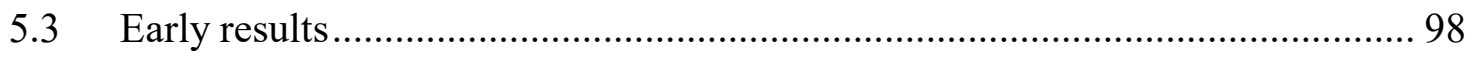

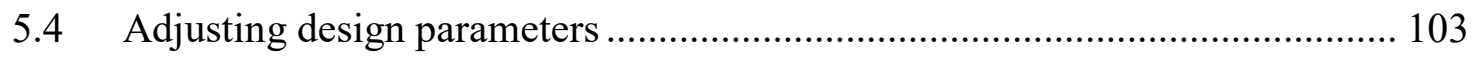

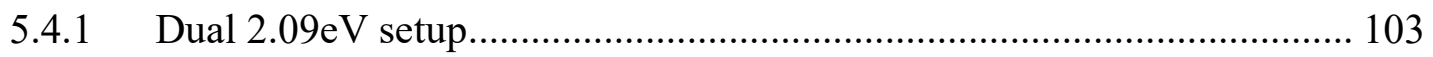

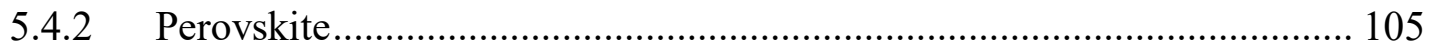

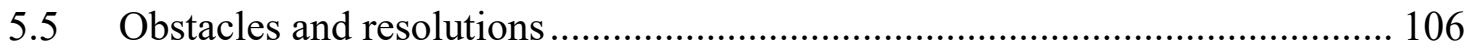

5.6 Restoring and improving resolution ................................................... 108

Chapter 6 CONCLUSION AND FURTHER WORK......................................... 114

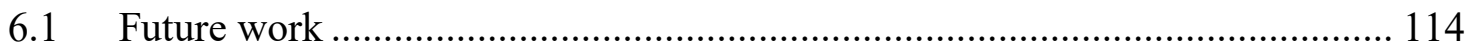

6.1.1 Hardware/Software improvements ................................................... 114 


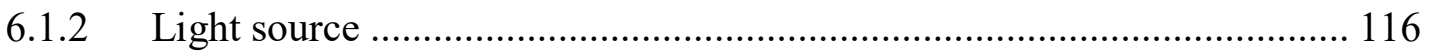

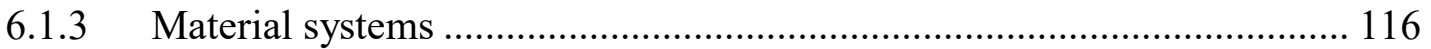

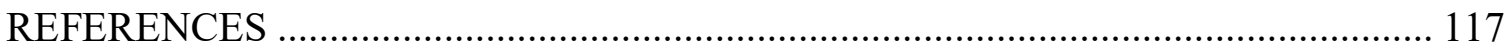

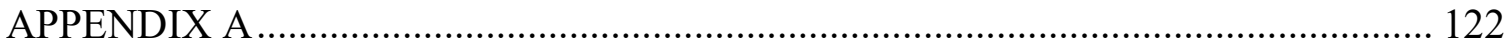

Capacitive Photocurrent - Stimulated Emission Depletion (CPC-STED) Standard

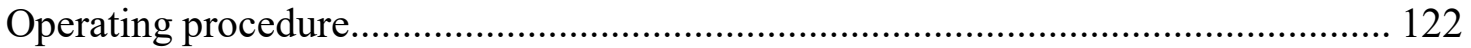

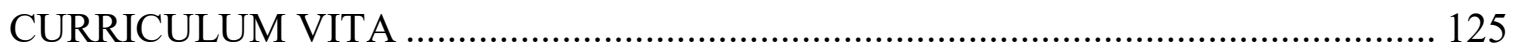




\section{LIST OF FIGURES}

FIGURE

PAGE

Figure 1.1: Various methods of photoluminescence (a) direct relaxation with photon emission (b) relaxation with one non-radiative step (c) relaxation with two non-radiative

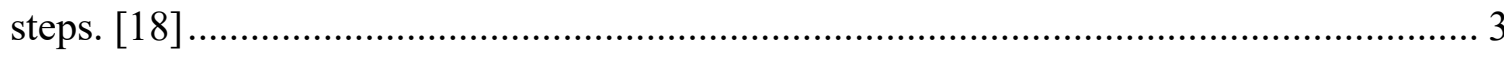

Figure 1.2: High-performance custom front-face excitation spectrofluorometer [12] ....... 4

Figure 1.3: Simple diagram of a photocurrent measurement setup. [9]........................... 5

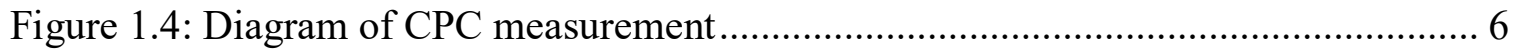

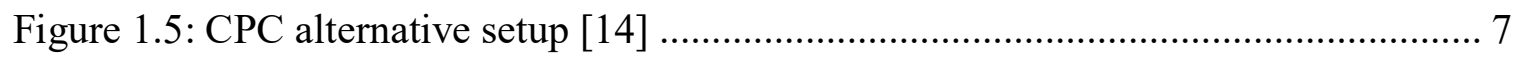

Figure 1.6: Simple layout of an Atomic Force Microscope [13] ................................... 8

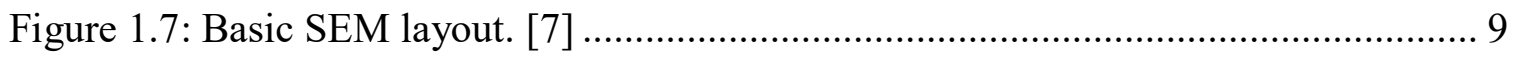

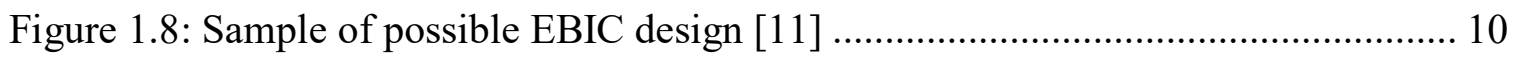

Figure 1.9: SEM image of the InSb-InAs NW device (a) and its EBIC image at $100 \mathrm{~K}$ (b).

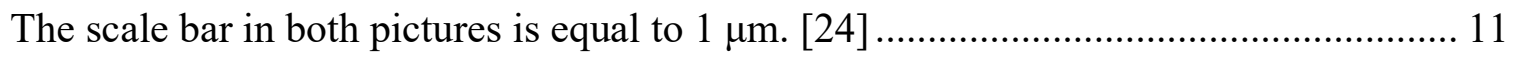

Figure 1.10: Comparison of resulting image for Rayleigh versus Abbe limited systems.

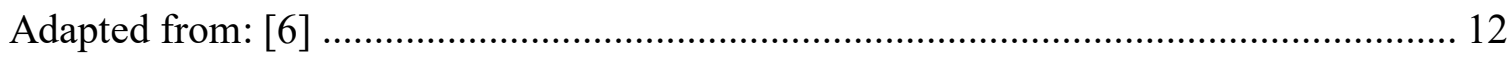

Figure 1.11: PALM based technique where: two frequencies are illuminating the sample for readout (a) and activation (b) and then fluoresce (c) are localized (d), bleached (e) and repeated (f) until a sufficient number of molecules have been detected to build a

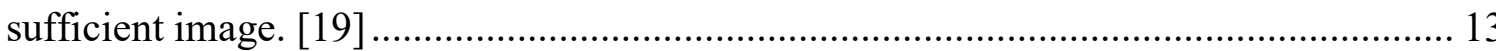


Figure 1.12: (a) Combination of depletion and excitation beams to enhance spatial resolution, (b) bright and dark states in normal microscopy and STED microscopy. ...... 15

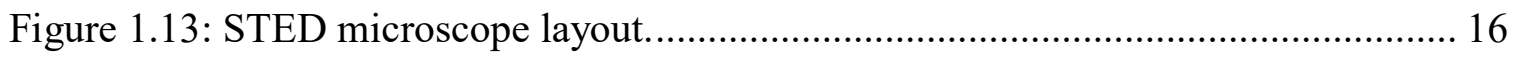

Figure 1.14: The scanning capacitive photocurrent technique used for STED electrical imaging. The voltage on a floating probe with (a) no illumination and (b) with illumination exciting charge from optically absorbing defect states. (c) Measurement setup including sample holder for STED electrical imaging (d) Photo showing objective and

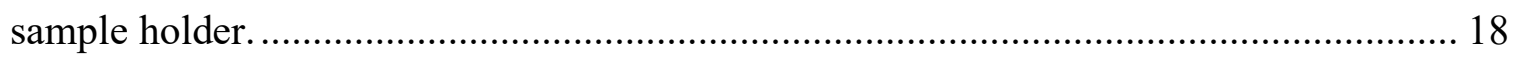

Figure 2.1: Hurricane system external and internal layout. [5] .................................. 20

Figure 2.2: Stretching and compressing to prevent damage to optics with high power final

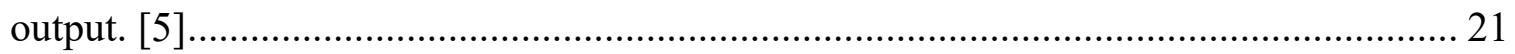

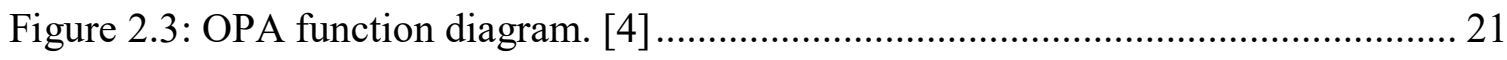

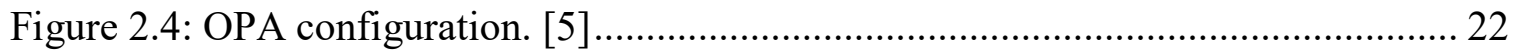

Figure 2.5: Configuration of OPA for wavelength extension. [4] ............................... 23

Figure 2.6: Configuration for FHG of the signal. [4] ............................................. 24

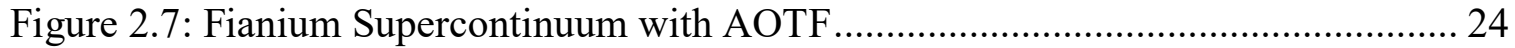

Figure 2.8: Functional diagram of a supercontinuum fiber light source [3] .................. 25

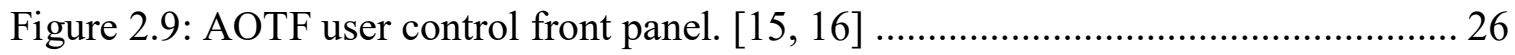

Figure 2.10: Single wall nanotube CPC investigation. [21] ...................................... 27

Figure 2.11: Comparison of SWNT CPC to absorbance. [21] ................................... 28

Figure 2.12: CPC spectra of three graphene-oxide samples with peaks at $0.7 \mathrm{eV}, 1.6 \mathrm{eV}$,

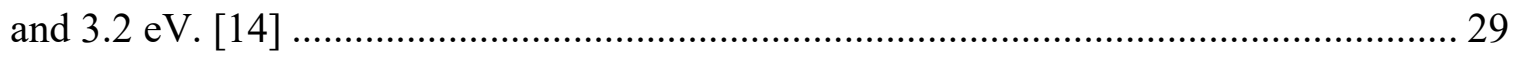

Figure 2.13: XPS of graphene-oxide (GO) and reduced graphene-oxide (RGO) [14] .... 30 
Figure 2.14: Absorbance and luminescence for P3HT (a) and MDMO0PPV (b) and the

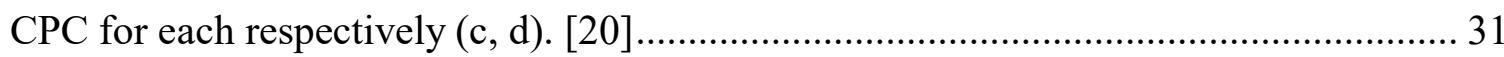

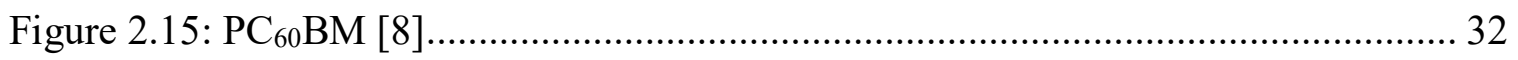

Figure 2.16: Collecting CPC signal of PCBM (a), with the effect of biasing (b) [2] ....... 33

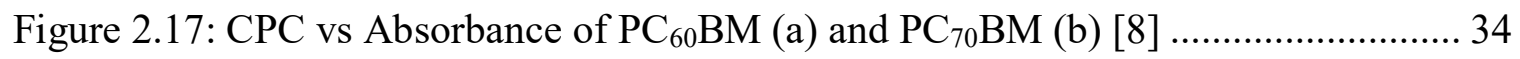

Figure 2.18: Effect of including insulating LiF layer. (a) Spectrum, (b) effect of increasing $\mathrm{LiF}$ thickness on $\mathrm{CPC}$ signal at $0.7 \mathrm{eV}$ (c) same at $1.5 \mathrm{eV}$ [2] ..................... 35

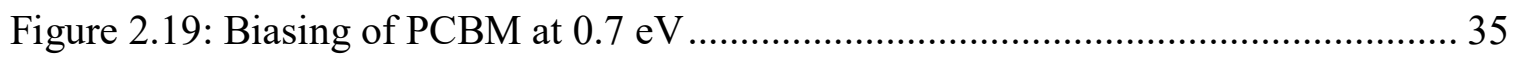

Figure 2.20: Subgap bias dependence with Fowler-Nordheim fit ............................... 36

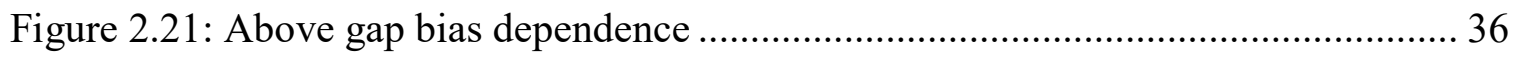

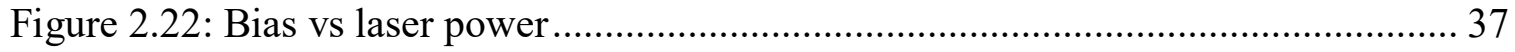

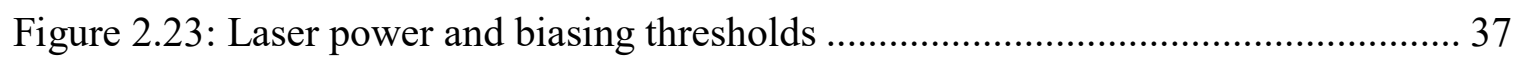

Figure 2.24: Band diagram for ITO/PCBM charge-transfer process............................ 38

Figure 2.25: Calculated absorbance spectra for polymeric fullerene chains. The solid red line shows the ky polarized absorption and the dashed black line shows the kx polarized absorption. (A) Neutral C60 chain. (B) C60 chain with one added electron. (C) C60 chain with two added electrons. (D) Neutral C70 chain. (E) C70 chain with one added electron.

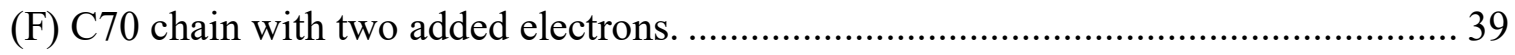

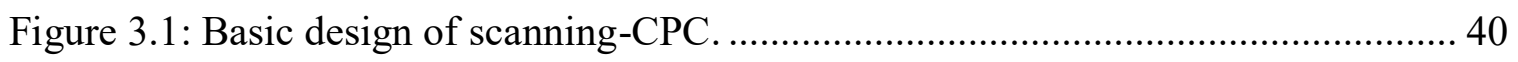

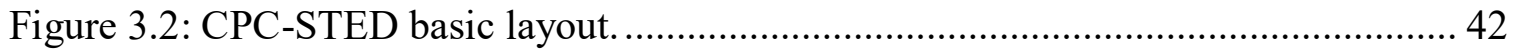

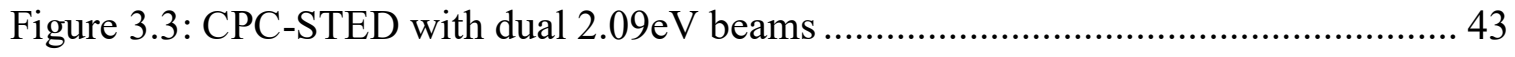

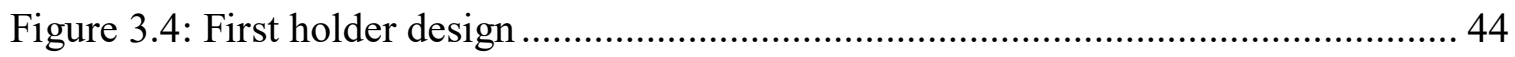

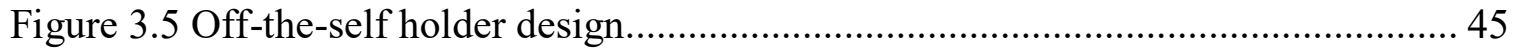




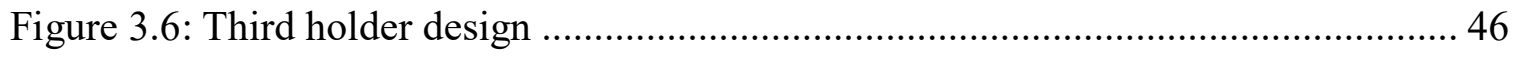

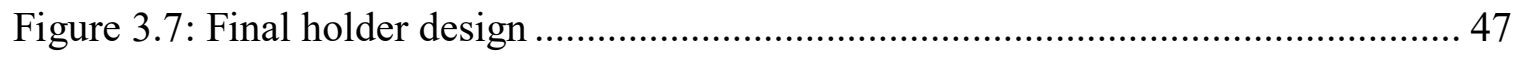

Figure 3.8: LabView front panel............................................................................. 49

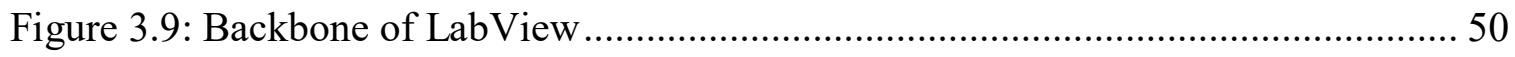

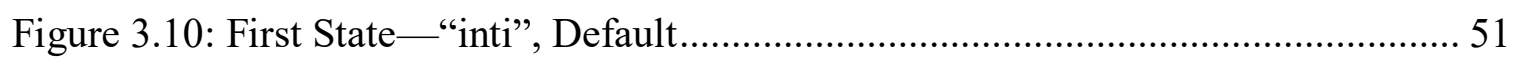

Figure 3.11: Second State—"waiting for begin" ....................................................... 52

Figure 3.12: Third state_-begin_x" ..................................................................... 52

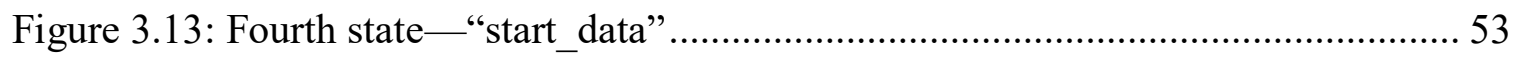

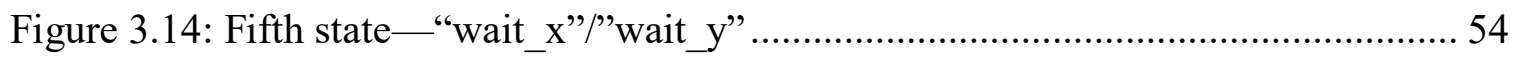

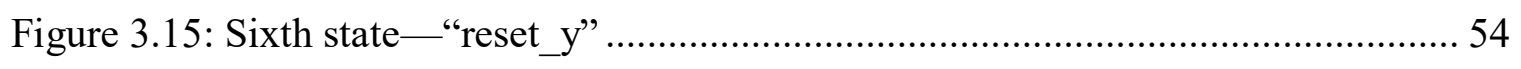

Figure 3.16: Seventh state—“end" .......................................................................... 55

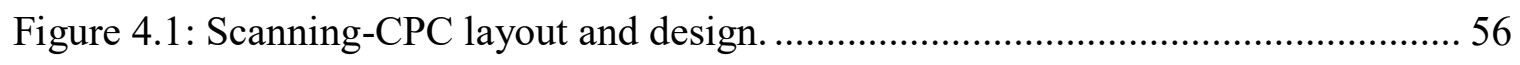

Figure 4.2: CPC signal as a function of chopper frequency. ........................................ 57

Figure 4.3:CPC dependence on $2.54 \mathrm{eV}$ excitation beam power. ................................... 57

Figure 4.4: Comparisons of nominal (software reported) laser power versus the sample surface measured power for the $2.54 \mathrm{eV}$ beam and $2.09 \mathrm{eV}$ beam (inset). ...................... 58

Figure 4.5: CPC image of a PCBM clump with a $2.54 \mathrm{eV}$ light source. .......................... 59

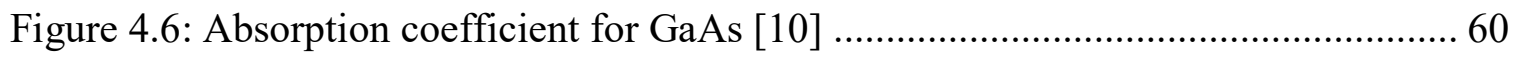

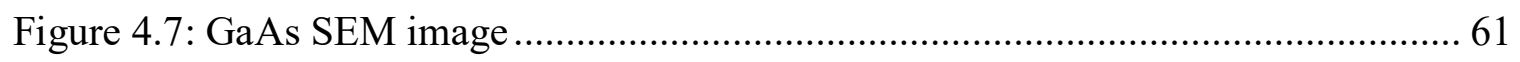

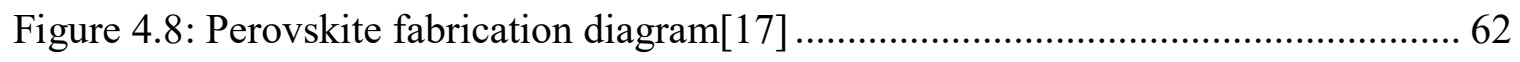

Figure 4.9: Optical image of perovskite deposited films. [17] ........................................ 62

Figure 4.10: External quantum efficiency and integrated short circuit current density of

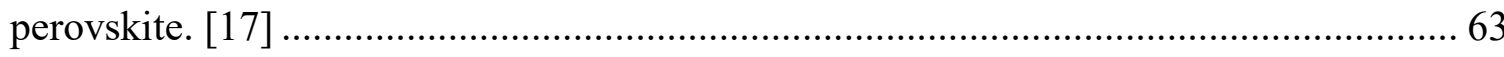




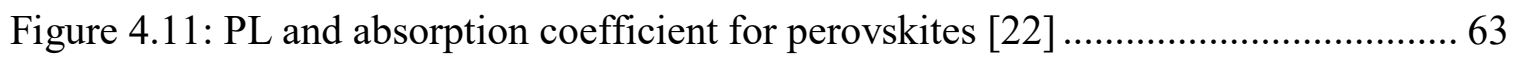

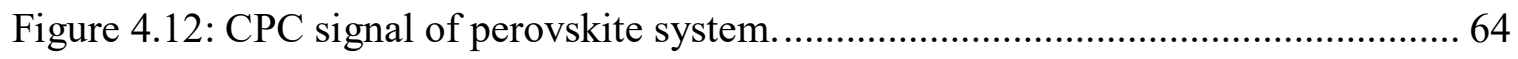

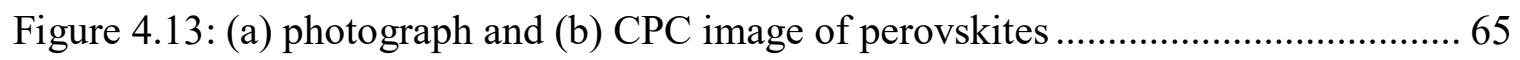

Figure 4.14: Burnt large perovskite formation after CPC scan. ................................... 66

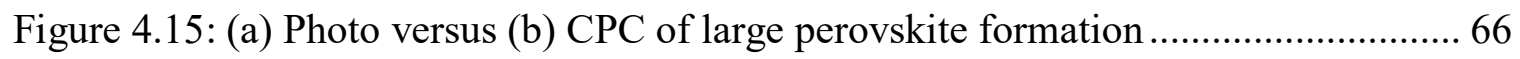

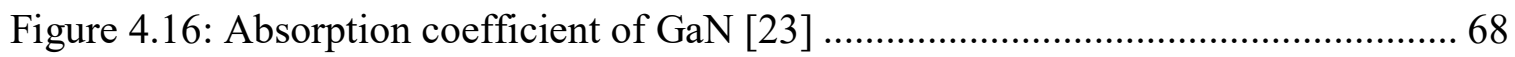

Figure 4.17: Comparison of GaN photoluminescence and CPC spectrum..................... 68

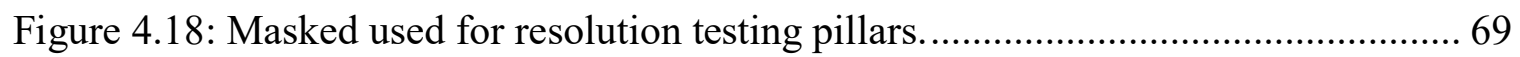

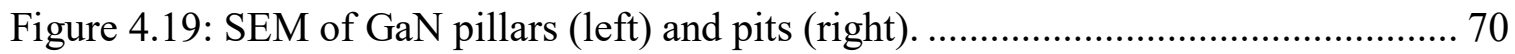

Figure 4.20: SEM (a) and CPC (b) image of shallow GaN pits and pillars SEM (b), CPC

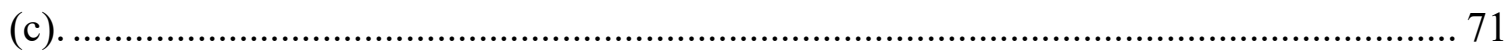

Figure 4.21: Short etch time (shallow) versus long etch time (deep) GaN pits............... 72

Figure 4.22: SEM (a), 2D CPC (b), and 3D CPC of a deep GaN pit............................. 72

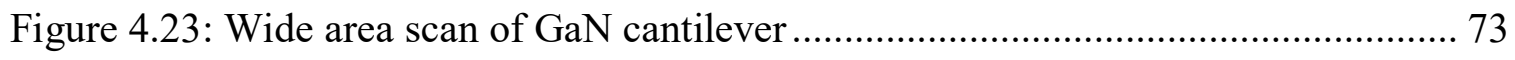

Figure 4.24: Line scan of CPC signal from edge of contact to 350um away from contact.

Figure 4.25: GaN 10um pillar piezoelectric electric field ..................................... 75

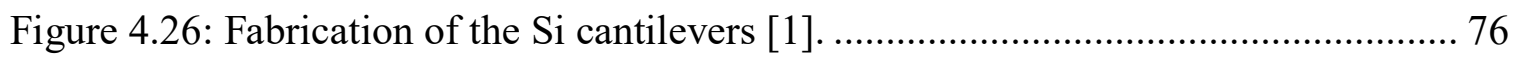

Figure 4.27: Top down photo of Si (a) wide view and (b) close up. [1]........................ 76

Figure 4.28: Effect of stress on CPC signal in a silicon cantilever.............................. 77

Figure 4.29: Diagram of bias testing GaN samples. ............................................... 78

Figure 4.30: Effects of external bias on CPC signal of GaN pillars ............................ 78

Figure 4.31: IV curves of diodes made from Sample 1 and Sample 2. ............................. 80 
Figure 4.32: CPC of pillar edge between different defect densities 80

Figure 4.33: Etched time comparisons, tall versus short pillars. 81

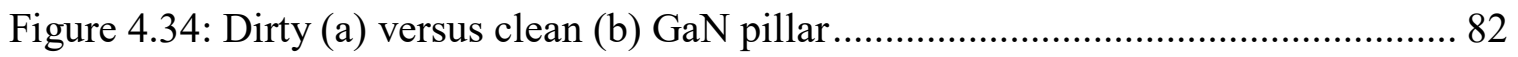

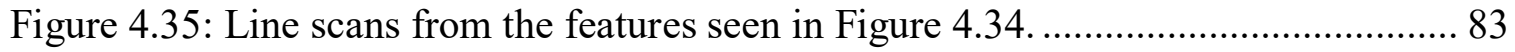

Figure 4.36: Unpassivated (a) versus passivated (b) GaN pillars ................................ 84

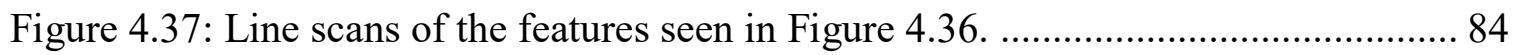

Figure 4.38: Top (a) and bottom (b) contact location on Silicon Cantilevers ................. 86

Figure 4.39: Photo of circular contact where images were taken (a) inside (b) outside, and

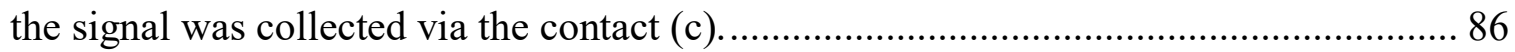

Figure 4.40: Pillar asymmetry seen in GaN pillars depends on position with respect to the

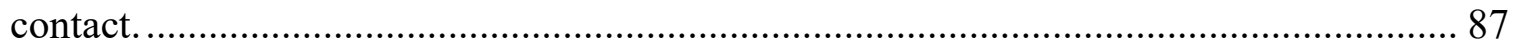

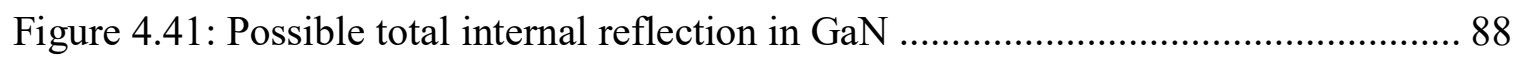

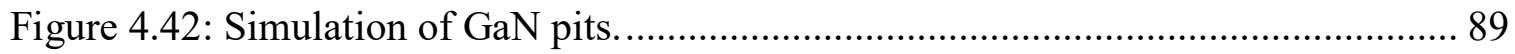

Figure 5.1: STED microscopy beam layout. (a) excitation beam only, (b) toroidal depletion beam, (c) overlapping of gaussian depletion and gaussian excitation beam, and (d) collinear toroidal depletion beam and gaussian excitation beam. ........................... 91

Figure 5.2: Diagram showing the importance of $\mathrm{I}_{\mathrm{s}}$ and depletion intensity. ................... 93

Figure 5.3: Theoretical limits of CPC-STED effective spot size, using a NA=0.80

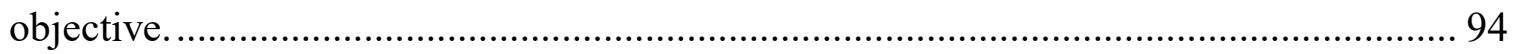

Figure 5.4: Fit of depletion intensity with CPC signal. ........................................... 94

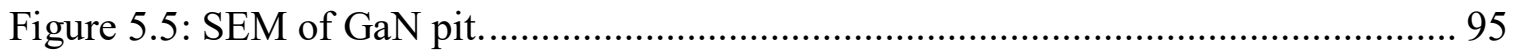

Figure 5.6: Bleach test of GaN pits. (a) bleached image, (b) excitation beam only image,

(c) CPC-STED image, and (d) line scans form each image. .................................... 96 
Figure 5.7: Comparing contact location with CPC-STED functionality. Outside the contact with (a) excitation only and (b) CPC-STED, inside the contact with (c) excitation only and (d) CPC-STED, and (e) line scan comparisons. 97

Figure 5.8: GaN pits with excitation only (a) and CPC-STED (b) with a line scan (c) across both. 98

Figure 5.9: More GaN pits with excitation only (a) and CPC-STED (b) with a line scan (c) across both. 98

Figure 5.10: (a) Minimum depletion power (b) Maximum depletion power image of pillars in $\mathrm{GaN}$ 98

Figure 5.11: Lines Scans of the GaN pillar features in Figure 5.10 99

Figure 5.12: A comparison of (a) excitation only, (b) low excitation power CPC-STED, (c) high power excitation CPC-STED, and (d) line scans of each image. 100 Figure 5.13: (a) Photo of pillars fabricated in a GaN wafer, with a dotted line along the line scan path and (b) line scans for excitation only in red and CPC-STED in blue..... 101 Figure 5.14: RayOptics simulation of the (a) $2.54 \mathrm{eV}$ excitation and (b) $2.09 \mathrm{eV}$ depletion beam path through the wall of a GaN pit. 102

Figure 5.15: Bleach test of dual $2.09 \mathrm{eV}$ beams with (a) $2.09 \mathrm{eV}$ excitation only (b) dual bleaching $2.09 \mathrm{eV}$, and (c) line scans through the center of the images. 103 Figure 5.16: (a) $2.09 \mathrm{eV}$ excitation only, (b) dual $2.09 \mathrm{eV}$ CPC-STED, and (c) line scans across the GaN pillars 104

Figure 5.17: (a) $2.54 \mathrm{eV}$ excitation only and (b) CPC-STED of perovskites. 105

Figure 5.18 :Line scan across the images in Figure 5.17 106 
Figure 5.19: Photographs from the camera on the microscope showing proper alignment.

Figure 5.20: Bleached, excitation only, and CPC-STED image of GaN pits with new stage and software. 108

Figure 5.21: Scanning-CPC (a) and CPC-STED (b) image of resolution testing GaN

pillars

Figure 5.22: GaN pillars taken via (a) scanning-CPC and (b) CPC-STED, with line scans

(c) of the separations. 110

Figure 5.23: (a) scanning-CPC image, (b) CPC-STED image, and (c) line scans across both with the excitation only line trace in blue and the CPC-STED line trace in red.... 111 Figure 5.24: Line scans of the GaN pit seen in Figure 5.23 with varying values for R. 112 Figure 6.1: Effect of numerical aperture on theoretical minimum spot size with a 2.09 $\mathrm{eV} / 2.54 \mathrm{eV}$ system, using a $\mathrm{R}=3.67$ value. 115 


\section{CHAPTER 1 INTRODUCTION}

\subsection{Motivations}

Traditional microscopy seeks to improve resolution, but has been traditionally limited by the diffraction qualities of the optics used. In 2014, the Nobel Prize in Chemistry was awarded to Stefan Hell for the development of a super resolution microscope via the Stimulated Emission Depletion (STED) technique. This award was for Dr. Hell's work in improving resolution beyond the limits of an optic's diffraction capabilities by using a novel system design. Since that point much work worldwide has been devoted to achieving similar imaging results at the super resolution level because super resolution can help in probing smaller and smaller structures, $>5 \mathrm{~nm}$, providing information on a variety of systems. This dissertation investigated a new way of employing the STED technique to create images based on the electronic response of a sample.

The Alphenaar group at the University of Louisville has been developing and using the Capacitive Photocurrent (CPC) technique for probing electronic behavior. This technique has been used for material property analysis, but this dissertation will discuss how the $\mathrm{CPC}$ technique was developed into a two-dimensional scanning technique. Once the scanning-CPC technique was implemented, we sought to employ the physics underlining the STED technique, allowing for us to accomplish super resolution in electronic process imaging, creating the CPC-STED technique. 
The remainder of Chapter 1 discusses various one-dimensional and twodimensional techniques. Chapter 1 also discusses other super resolution techniques and how they apply to our new CPC-STED technique, finally discussing a brief overview of the CPC-STED technique. Chapter 2 is a review of the various CPC systems as well as discussion on the body of work done using the CPC technique, both done by others and myself. Chapter 3 discusses the design of the optical layout, hardware components, and software relating to the scanning-CPC and CPC-STED development. Chapter 4 discusses the results of the scanning-CPC technique. Chapter 5 discusses the results of the CPCSTED technique. Finally, Chapter 6 discusses the conclusions of this work as well as possible avenues for future work.

\subsection{Spectroscopy}

This section will discuss various techniques of fixed-point spectroscopy. A fixedpoint analysis measures the response of a sample at a single point and generates a onedimensional data set. The following spectroscopy techniques discussed measure the optical or electrical response of a sample when exposed to electromagnetic radiation, typically a monochromatic light source. When incident light excites electrons from lower energy states to higher energy states, this electron relaxation creates additional current, light, or heat. This result means that a range of wavelengths can be used to provide information on the electron structure of a sample. The technique we developed, CPCSTED, combines various elements of a variety of different spectroscopy techniques. The techniques described here are fluorescence spectroscopy, photocurrent spectroscopy, and finally the technique our group has developed, Capacitive Photocurrent Spectroscopy (CPC). 


\subsubsection{Absorbance spectroscopy}

Fluorescence spectroscopy probes the electronic structure of a sample by exciting electrons from a lower to higher state and analyzing the photons emitted as the electron returns to its lower state. In Figure 1.1 we have incident light exciting an electron to a higher state, this electron then (a) returns directly to the lower state, emitting a photon, (b) decays non-radiatively and then returns to the lower state and emits a photon, and (c)

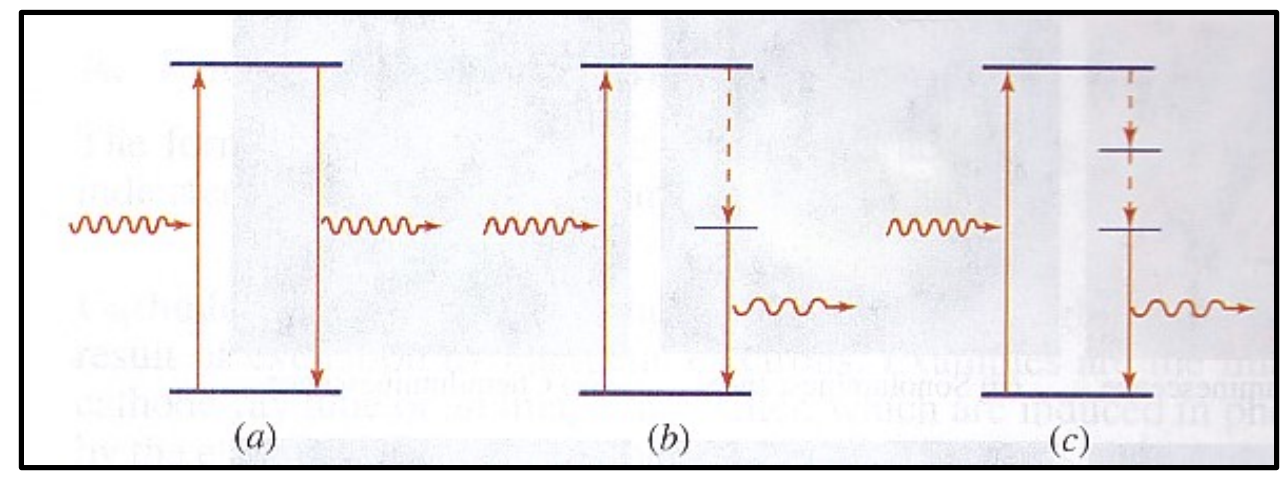

Figure 1.1: Various methods of photoluminescence (a) direct relaxation with photon emission (b) relaxation with one non-radiative step (c) relaxation with two non-radiative steps. [18]

undergoes multiple nonradiative processes before returning to its lower state and emitting a photon. This process occurs rapidly with a typical fluorescence lifetime of $\sim 10 \mathrm{~ns}$ [25]. With excitation induced as follows, where $\mathrm{S}_{0}$ is the ground state, $h v$ is the light's energy, $\mathrm{S}_{1}$ is excited state:

$$
S_{0}+h v_{\text {excitation }} \rightarrow S_{1}
$$

and fluorescent emission:

$$
S_{1} \rightarrow S_{0}+h v_{\text {emission }}+\text { vibrational relaxation(heat) }
$$

This identity provides information on the electronic states available as well as transitions that are typical of a specific molecule or material. An emitted photon has a specific wavelength that depends on both the material excited and the excitation wavelengths 
used. With an excitation wavelength providing a specific energy, E, and wavelength, $\lambda$,

$$
E=h v=h c / \lambda
$$

then with a material of a given ground state energy, $E_{g}$, will emit a particular wavelength that depends on the energy of the excited state, $\mathrm{E}_{\mathrm{e}}$, with

$$
E_{e}-E_{g}=h v
$$

we have an emitted wavelength, $\lambda_{\mathrm{e}}$ given by,

$$
\lambda_{e}=h c /\left(E_{e}-E_{g}\right)
$$

Figure 1.2 is a schematic for a excitation spectrofluorometer. This system uses a white light source that is focused to maximize power. A monochromator selects certain wavelengths from the chopped white light. The chopped light is split so that a reference can be collected. The light is then focused onto the sample in the front facing flow cell as shown the diagram. The light the sample emits is focused by a concave mirror, filtered, and then collected by another monochromator and photomultiplier tube (PMT). While

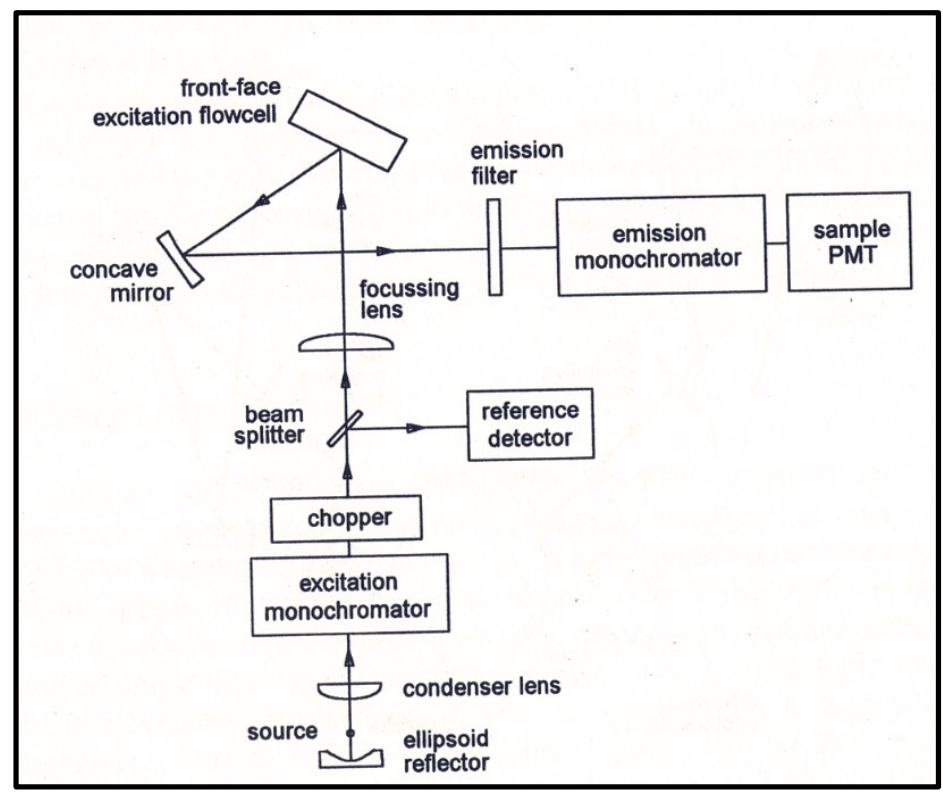

Figure 1.2: High-performance custom front-face excitation spectrofluorometer [12] 
there are other styles of fluorescence spectroscopy systems available, they all follow the same basic principle.

\subsubsection{Photocurrent spectroscopy}

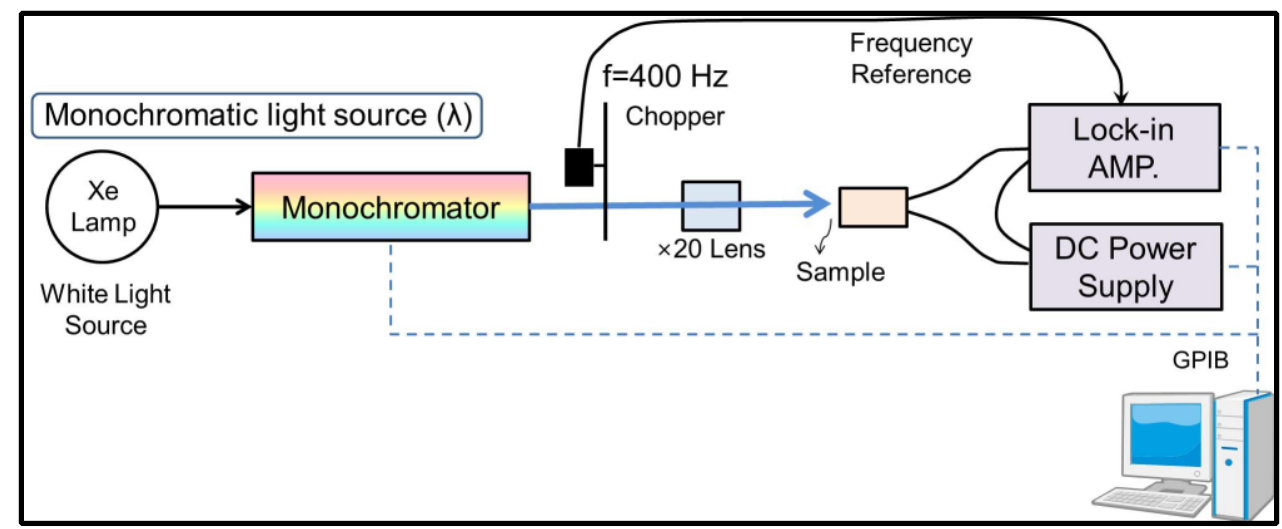

Figure 1.3: Simple diagram of a photocurrent measurement setup. [9]

Photocurrent spectroscopy probes the electronic structure of materials by measuring the current generated after the material is exposed to electromagnetic radiation. Free carriers generated by the light move in response to local fields, generating current [26]. As seen in Figure 1.3, the requirements for a photocurrent measurement system are relatively simple, a white light source, a monochromator, a beam modulator, and focusing components. The chopped monochromatic light is focused onto the sample where a lock-in improves signal integrity and a DC power supply helps in biasing the sample to improve signal strength. The photocurrent measurement system shown in Figure 1.3 was used to evaluate blue LEDs [9].

Scanning samples across an array of energies can determine material properties. This technique is frequently used in research involving solar cells because it helps predict solar cell function and efficiency. The photocurrent can be generalized as follows,

$$
I_{p h}=\eta P_{o p t} \frac{q}{h v}
$$


where $I_{p h}$ is the photocurrent, $P_{\text {opt }}$ is the optical power of the incident beam, and $\eta$ is the internal quantum efficiency (ratio of number electron-hole pairs contributing to the photocurrent to the number of photons absorbed by the sample) [9]. Where the quantum efficiency is a material property leaving only the photon flux, the number of photons per second per meter-squared, as the adjustable parameter, meaning either the light source's intensity can be increased.

This technique does require free carriers, and the carriers must be able to be collected by the contacts. This requirement means it is not suitable for some types of samples. The next sections will discuss how the CPC technique overcomes the free carrier requirements.

\subsubsection{Capacitive Photocurrent (CPC) spectroscopy}

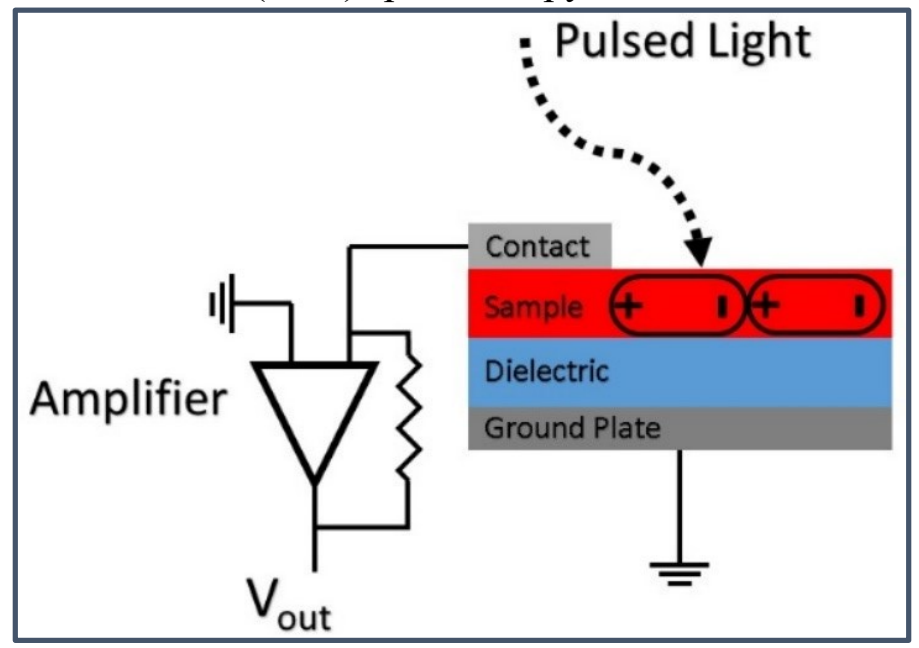

Figure 1.4: Diagram of CPC measurement

Capacitive-photocurrent (CPC) spectroscopy is the measurement of the AC signal of the current generated from the electron-hole separation generated in a sample. This signal generation is done by setting up the sample in a similar way to a metal-insulatorsemiconductor (MIS) photo detector, where the semiconductor is replaced with the 
sample as shown in Figure 1.4. With a modulated light source, a displacement current is generated across the capacitor formed with the sample and a ground plate. The CPC technique is a measurement of the samples absorption as well as the electron-hole separation generated in the sample. CPC also allows for non-destructive spectroscopy and simple sample preparation. Often, it only requires a simple silver paste contact on the sample surface and mounting to the dielectric/insulator. In Figure 1.5, we have the layout used for testing graphene oxide. Since this is an AC measurement, it allows for using a lock-in to increase signal fidelity, reducing the signal to noise ratio

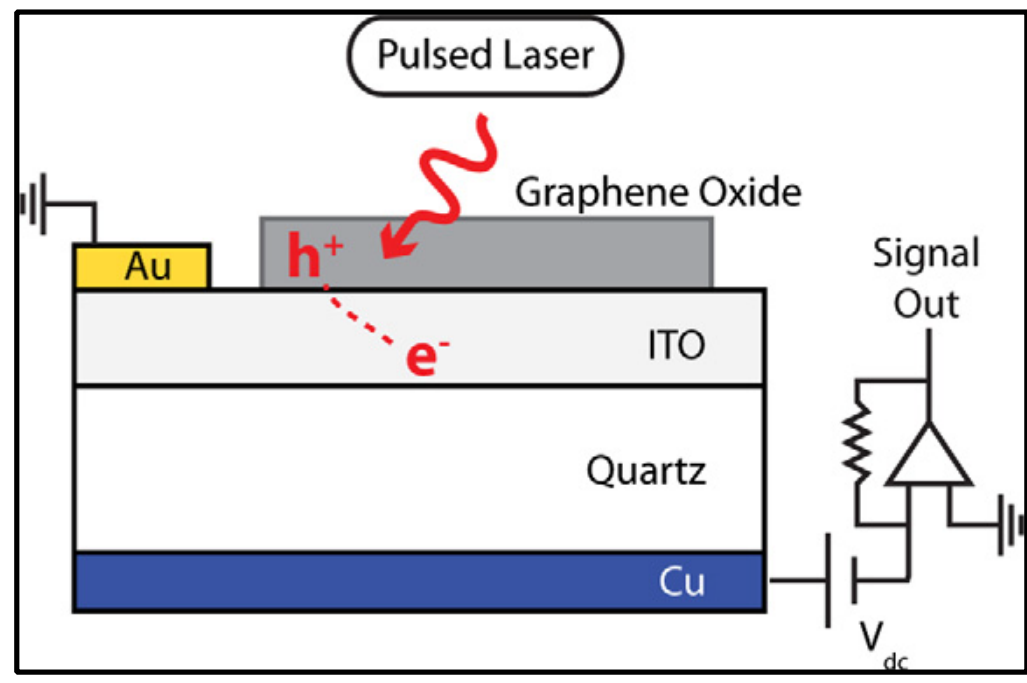

Figure 1.5: CPC alternative setup [14]

\subsection{D imaging}

Two-dimensional images can be created by using the fixed-point framework and moving either the sample or the interrogation beam and then collecting the intensity of the sample's response to generate an image of the sample with respect to its response. This 2D survey will also cover a topological focused technique, atomic force microscopy (AFM), as well as scanning electron microscopy (SEM), and electron beam-induced current (EBIC) imaging. Further discussion of fluorescence based two-dimensional 
imaging will be discussed in Section 1.4 with a survey of sub-diffraction techniques, including STED.

\subsubsection{Atomic Force Microscopy (AFM)}

Atomic force microscopy is an imaging technique that provides topological profiles of a sample's surface. The AFM uses a pointed cantilever that is moved across that samples surface with electrical, magnetic, or physical interactions. The surface topology causes the cantilever to deflect, moving a laser that is reflected off the back side of the cantilever. This lever has a tip, $t$, in Figure 1.6 that is drawn across the sample, $S$. With a laser, $L$, targeting the reflective back of a cantilever where we measure the deflection of the cantilever by correlating the reflected beam signal in the photodetectors, $P D_{1}$ and $P D_{2}$, this collected signal generates an image corresponding to the surface profile of the sample. AFM has trouble when the surface profile of a sample with sharp angles since they can impede the motion of the tip of the cantilever due to the tip's angle.

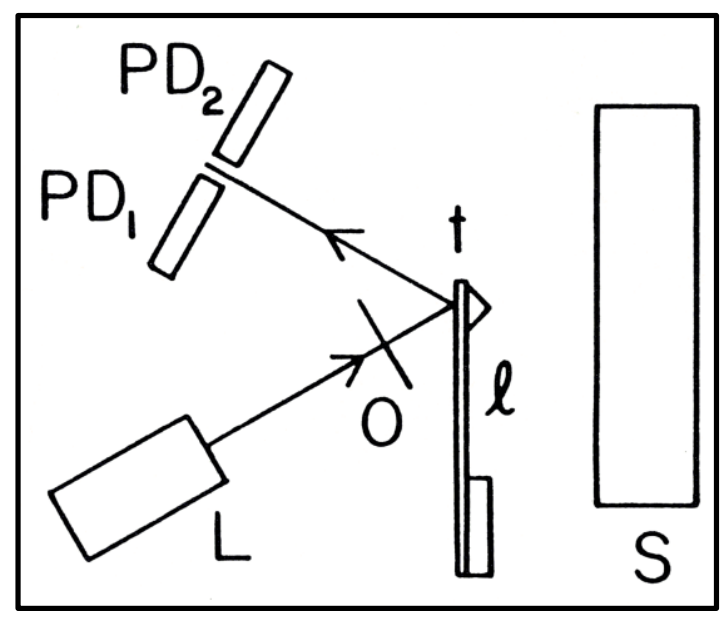

Figure 1.6: Simple layout of an Atomic Force Microscope $\lceil 137$

AFM measurements were used in our work creating a two-dimensional profile of the surface topology of materials later characterized using the scanning-CPC system. 
With accurate representations of a sample's physical topography, we can correlate these physical topographical profiles to the electrical images generated by the CPC signal.

\subsubsection{Scanning Electron Microscope (SEM)}

Scanning electron microscopy measure the interactions between a high-energy electron beam and a sample. Figure 1.7 shows a basic SEM design, with an electron gun having its beam focused onto a sample and the resulting reflected beams being collected. The SEM collects either the reflected electrons or the generated secondary electrons, this signal provides information about the sample's morphology, composition, and structure. The collected data is displayed as a $2 \mathrm{D}$ image where the z-axis is the intensity of the signal. This technique allows for images to have down to 1-2 $\mathrm{nm}$ resolution. This technique allows for collection of multiple resulting signals: secondary electrons, backscattered electrons, diffracted backscattered electrons, and photons. The system also requires a $\mathrm{HV}$ or UHV environment.

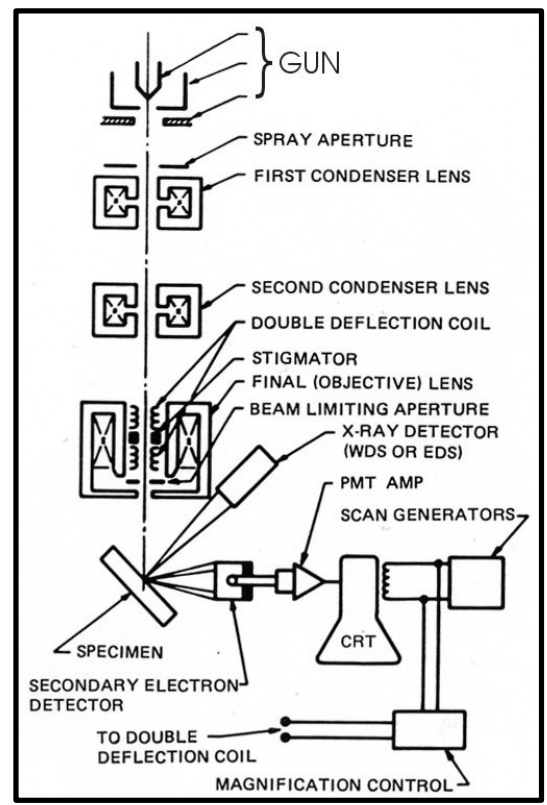

Figure 1.7: Basic SEM layout. [7] 
With the advantages this technique offers, we used it to examine the topological profiles of our semiconductor samples. This comparison allows for us to better correlate the images provided by the SEM with the images provided by the scanning-CPC and the CPC-STED. Some of the details provided by the SEM technique are used in Chapters 4 and 5 .

\subsubsection{Electron Beam-Induced Current (EBIC)}

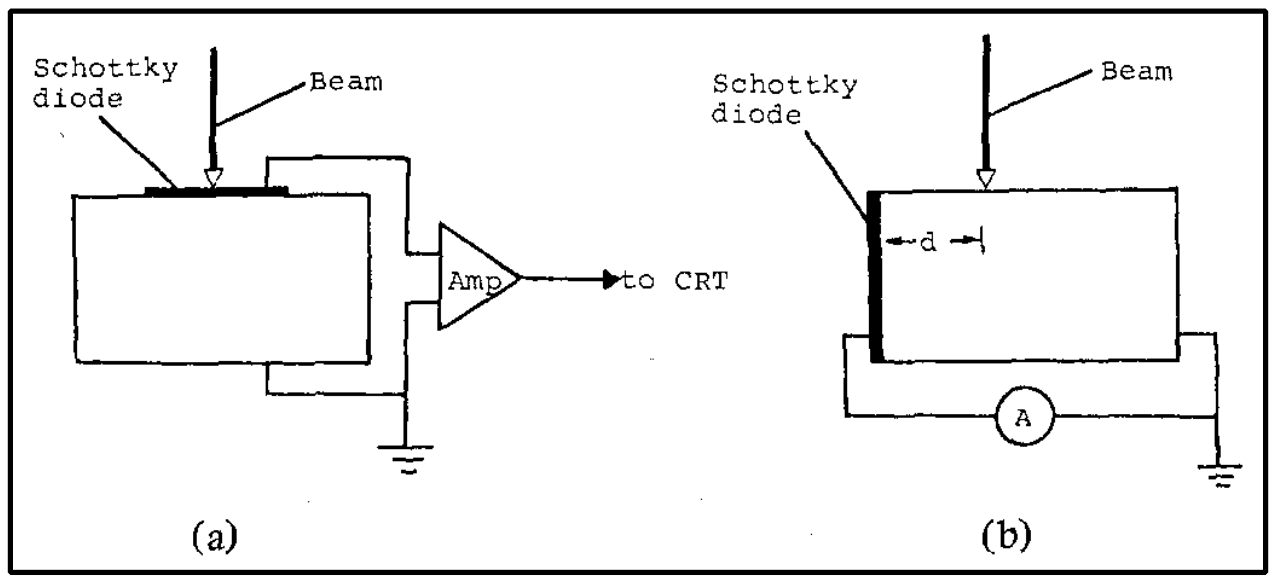

Figure 1.8: Sample of possible EBIC design [11]

Electron beam induced-current applies an electron beam to a sample and the current created is measured. The sample or beam can be moved to generate a twodimensional image that can be used to describe the material's properties. EBIC is done in a modified scanning electron microscope (SEM). This technique is for characterizations, such as locating $\mathrm{p}-\mathrm{n}$ junctions, detecting defects, or measuring minority carriers. EBIC relies on the internal electric field of a sample. The beam of electrons generates electronhole pair separation resulting in a measurable current.

This technique is valuable for understanding certain semiconductor systems as well as doing failure analysis and exploring solid-state physics. EBIC requires the 
modification of either a SEM or Scanning Transmission Electron Microscope (STEM) and a high vacuum (HV) or ultra-high vacuum (UHV) environment.

In Figure 1.9, we see a SEM image (a) inset on a scanning EBIC image (b). This scan shows how a InSb-InAs nanowire device has an unusual profile near the heterointerface because the signal switching from positive to negative. The EBIC technique is useful here since this is a measure of carriers collected, unlike CPC measurements that do not require carrier collection. This figure also shows how EBIC has an advantage by collecting the secondary electrons of a scan to build a topological image

(a) as well as an electronic response image (b).

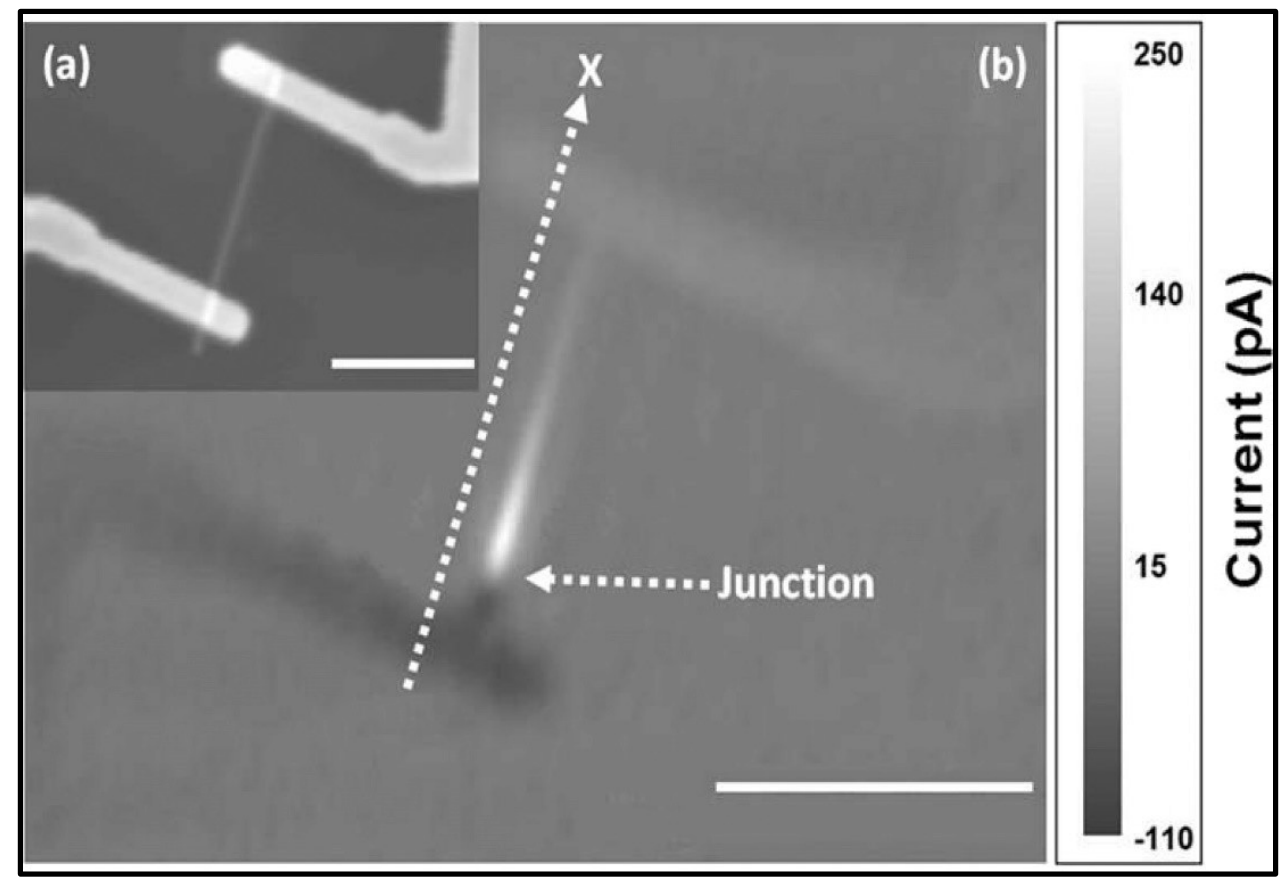

Figure 1.9: SEM image of the InSb-InAs NW device (a) and its EBIC image at $100 \mathrm{~K}$ (b). The scale bar in both pictures is equal to $1 \mu \mathrm{m}$. [24]

$1.4 \quad$ Sub-diffraction limited techniques

Traditional imaging techniques are limited by the minimum diameter of the light on the sample's surface. In traditional fluorescence microscopy, the maximum resolution is 
$\sim 200-300 \mathrm{~nm}$ [27]. This limit ( $d$ ) is controlled by an objective's numerical aperture (NA) and the wavelength of the light $(\lambda)$ given by the Abbe diffraction limit,

$$
d=\frac{\lambda}{2 N A}
$$

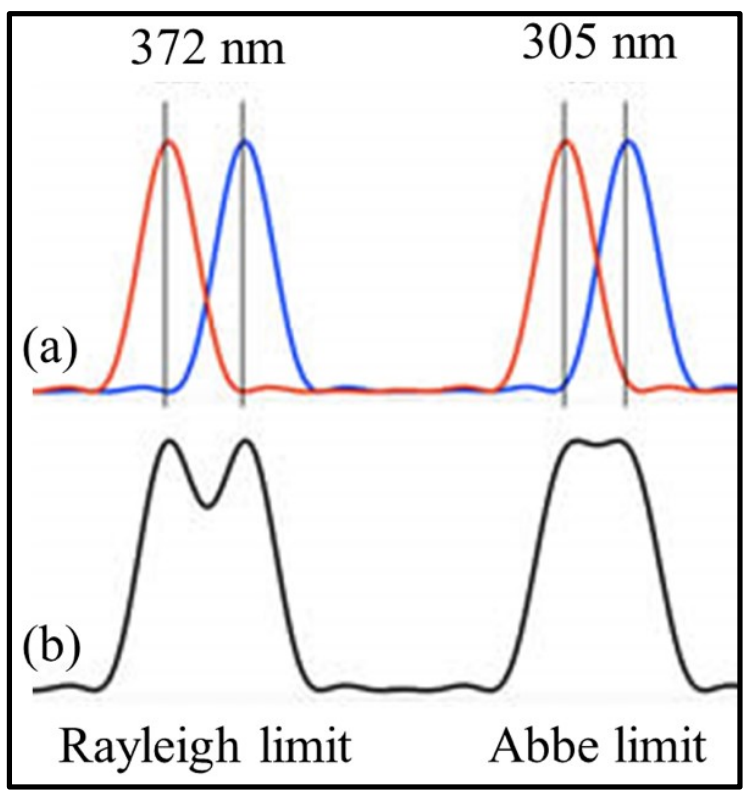

Figure 1.10: Comparison of resulting image for Rayleigh versus Abbe limited systems. Adapted from: [6]

This limitation means that we cannot resolve any separations or features below the diffraction limit $(d)$. We can see in Figure 1.10 how this limit (Abbe limit) cannot differentiate between two separate features (a) as seen in the resulting image in (b) when using a $2.54 \mathrm{eV}(488 \mathrm{~nm})$ beam through a $0.80 \mathrm{NA}$ objective. We must look to the Rayleigh criterion and Abbe's limit to determine that. The Abbe's limit is commonly referred to as the diffraction limit, the minimum spot size we can achieve, this minimum spot size is $305 \mathrm{~mm}$ with our objective and a $2.54 \mathrm{eV}$ excitation beam. The Rayleigh criterion is a systems minimum resolvable detail, given by

$$
d=\frac{0.61 * \lambda}{N A}=\frac{0.61 * 488 \mathrm{~nm}}{0.80}=372 \mathrm{~nm}
$$


therefore we can use this to examine features below these values to determine if super resolution is achieved, where the Rayleigh criterion is a more stringent definition of minimum resolvability.

This section briefly discusses various fluorescence optical techniques that break the diffraction limit. This resolution improvement can be accomplished via either deterministic or stochastic methods. Deterministic techniques, like STED, are precisely controlled with precise, expected responses. Stochastic techniques, like PALM/STORM, utilize the random nature of fluorescence in fluorophores. These techniques will be discussed in detail as well as why they can or cannot be applied to the CPC technique.

\subsubsection{PALM/STORM}

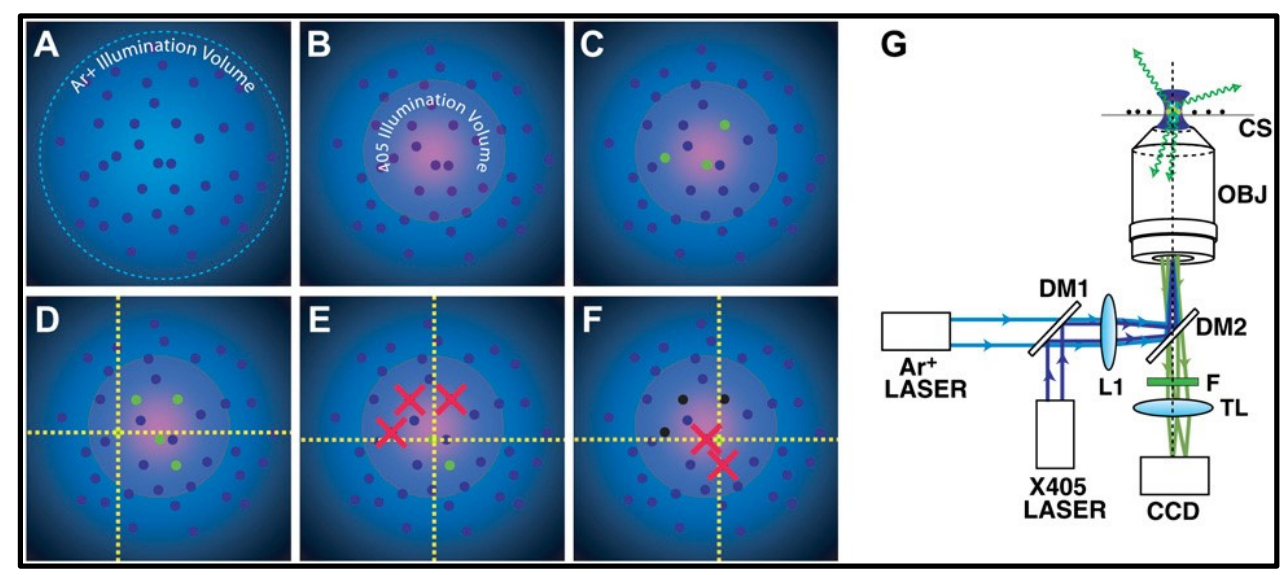

Figure 1.11: PALM based technique where: two frequencies are illuminating the sample for readout (a) and activation (b) and then fluoresce (c) are localized (d), bleached (e) and repeated (f) until a sufficient number of molecules have been detected to build a sufficient image. [19]

Photo-activated localization microscopy (PALM) and stochastic optical reconstruction microscopy (STORM) construct images by combining multiple frames where different fluorophores have emitted photon. Since a small portion of fluorophores 
have emitted in each frame by combining the frames, super-resolution images can be created. STORM utilizes the random nature of fluorescence of photo-switchable dyes by using a lower intensity beam and repeatedly exciting, identifying, recording, resetting a system to build a more complete picture. PALM also depends on the random nature of fluorescence, but uses photo-switchable protein fluorophores. In Figure 1.11, we see how the process works as well as the setup. Both techniques are widefield techniques rather than point scanning microscopy, avoiding the need of specific objectives. This sample analysis requires binding a dye or protein to the material of interest which increases the complexity of sample preparation.

For example, if you had two features that are too close to resolve, then by binding fluorophores to these structures you can expose a large area of a sample with excitation light. Then, with a fluorophore of known size, the points of single illumination can be recorded. Since the fluorophores do not fluoresce at the same time, these random emissions can be collected in successive pictures. A complete image can be built that achieves super resolution by repeating this procedure multiple times.

This technique is not suitable for applying the CPC technique. The CPC technique is much more deterministic by directly exciting the material that provides a signal. Therefore, other techniques can be compared to the CPC technique for suitability for development of an augmented scanning-CPC system

\subsubsection{STED}

Stimulated Emission Depletion (STED) microscopy uses stimulated depletion of fluorophores by a secondary, higher powered, lower energy beam to create images with 


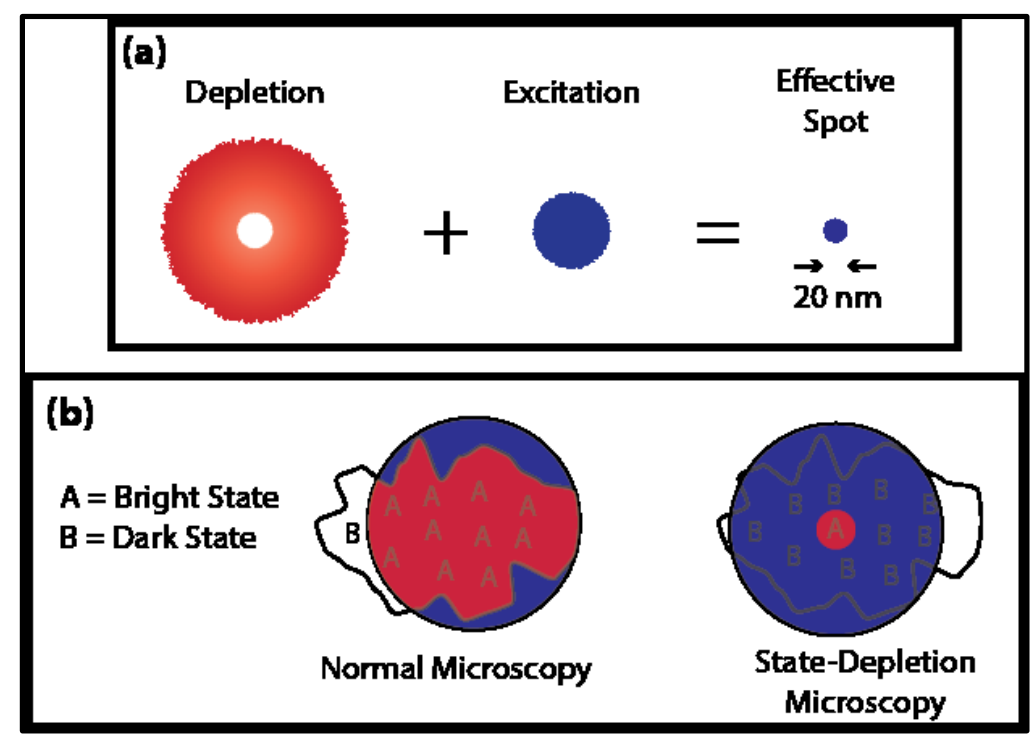

Figure 1.12: (a) Combination of depletion and excitation beams to enhance spatial resolution, (b) bright and dark states in normal microscopy and STED microscopy.

super-resolution. Figure 1.12 (a) shows the beam profiles used to accomplish this resolution improvement. With a toroidal beam stimulating depletion of fluorophores, the effective spot size is within the center of the toroidal depletion beam. This technique is deterministic since the effect of the depletion beam is tuned specifically and precisely applied, independent of random fluorescence response. This depletion is done by using a second lower energy (red shifted) beam with an energy between the excited and ground state that quickly returns the excited fluorophores to the ground state, keeping them 'dark.' This process means that the fluorescence signal can be strategically eliminated, a crucial component of STED microscopy. This result also means that the process of state depletions could be implemented in other techniques as will be discussed in Chapter 3 .

A typical system consists of using a laser as the light source for a confocal microscope. A depletion laser inhibits fluorescence emission by forcing excited fluorophores into their dark ground state, while an excitation laser excites fluorophores to 
induce spontaneous fluorescence. Overlapping a depletion and excitation beam then effectively reduces the spot size well below the diffraction limit. An effective spot sizes of $20 \mathrm{~nm}$ has been reported for living cells and $<5 \mathrm{~nm}$ for solid materials using the STED technique [27]. We have a toroidal depletion beam, creating a circular, effective excitation spot below the diffraction limit.

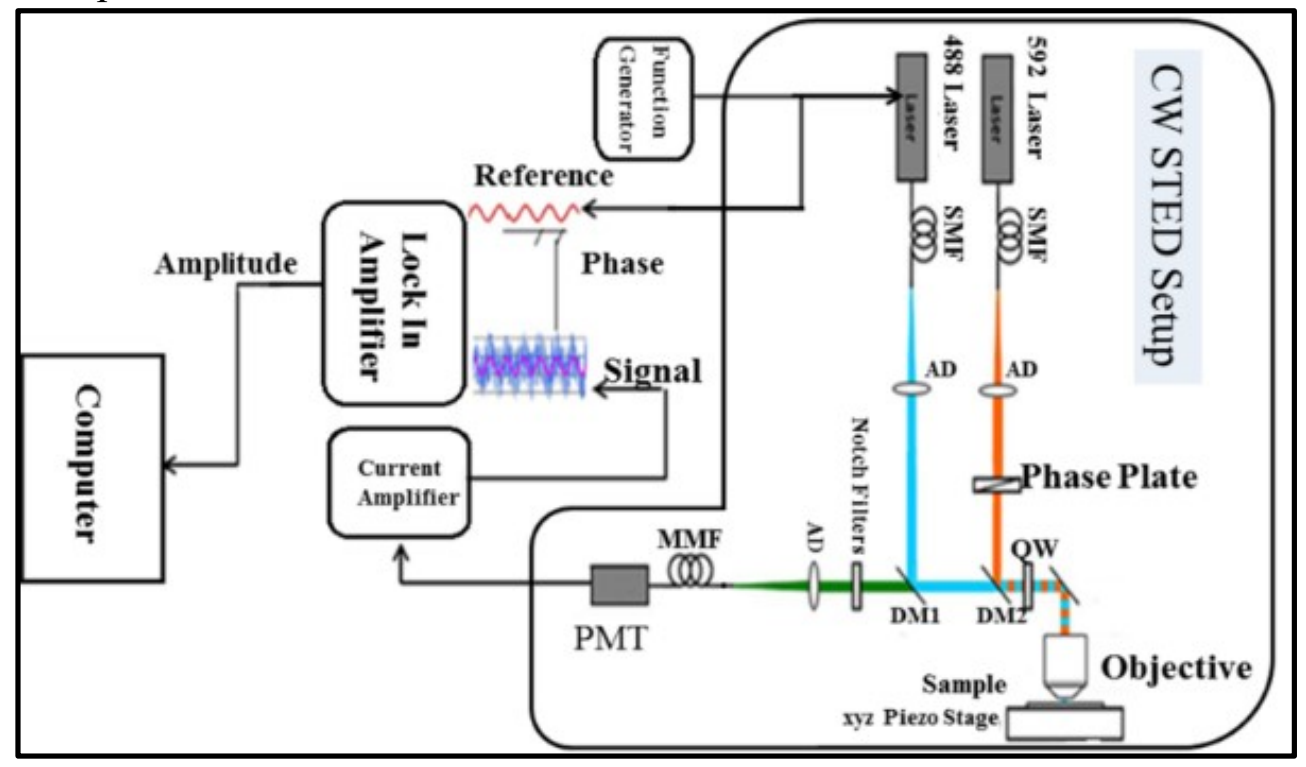

Figure 1.13: STED microscope layout.

Figure 1.13 shows the layout we used for our STED system. A $2.54 \mathrm{eV}(488 \mathrm{~nm})$ laser is the excitation beam and a $2.09 \mathrm{eV}(592 \mathrm{~nm})$ is the depletion beam. Both beams are injected into respective single-mode fibers (SMF). The output goes through collimating lenses (AD). Next, the depletion beam passes through a phase plate to create a toroidal pattern. We then overlap both beams so that they are collinear and steer them into the microscope and onto the sample. We collect the resulting fluorescence in a multimode fiber feeding into a photomultiplier (PMT) where the electric signal is amplified and sent to the computer, then processed into an image.

This technique is suitable for applying the CPC technique because it is deterministic, requiring the precise, controlled responses of a sample rather than 
depending on the random process of the sample. It is also conducive of the CPC techniques since most STED systems utilize open air scanning, rather than requiring vacuum or temperature controls. Since the $\mathrm{CPC}$ technique requires an alternating or pulsed light source for its AC measurements the depletion beam of the STED system could be maintained as a CW light source and the excitation beam could be mechanically chopped. Thereby, theoretically providing a technique for probing the electronic structure of a sample, without requiring carrier collection, and achieving super-resolution. The details of how this was implemented and the theoretical base for it will be discussed further in Chapters 3, 4, and 5. 


\subsection{Combining CPC with STED}

The CPC technique is an AC measurement which means we can take advantage of some of the physics that STED microscopy offers. By using a CW beam as the toroidal, depletion beam we can keep material in this area in a raised state, such that the

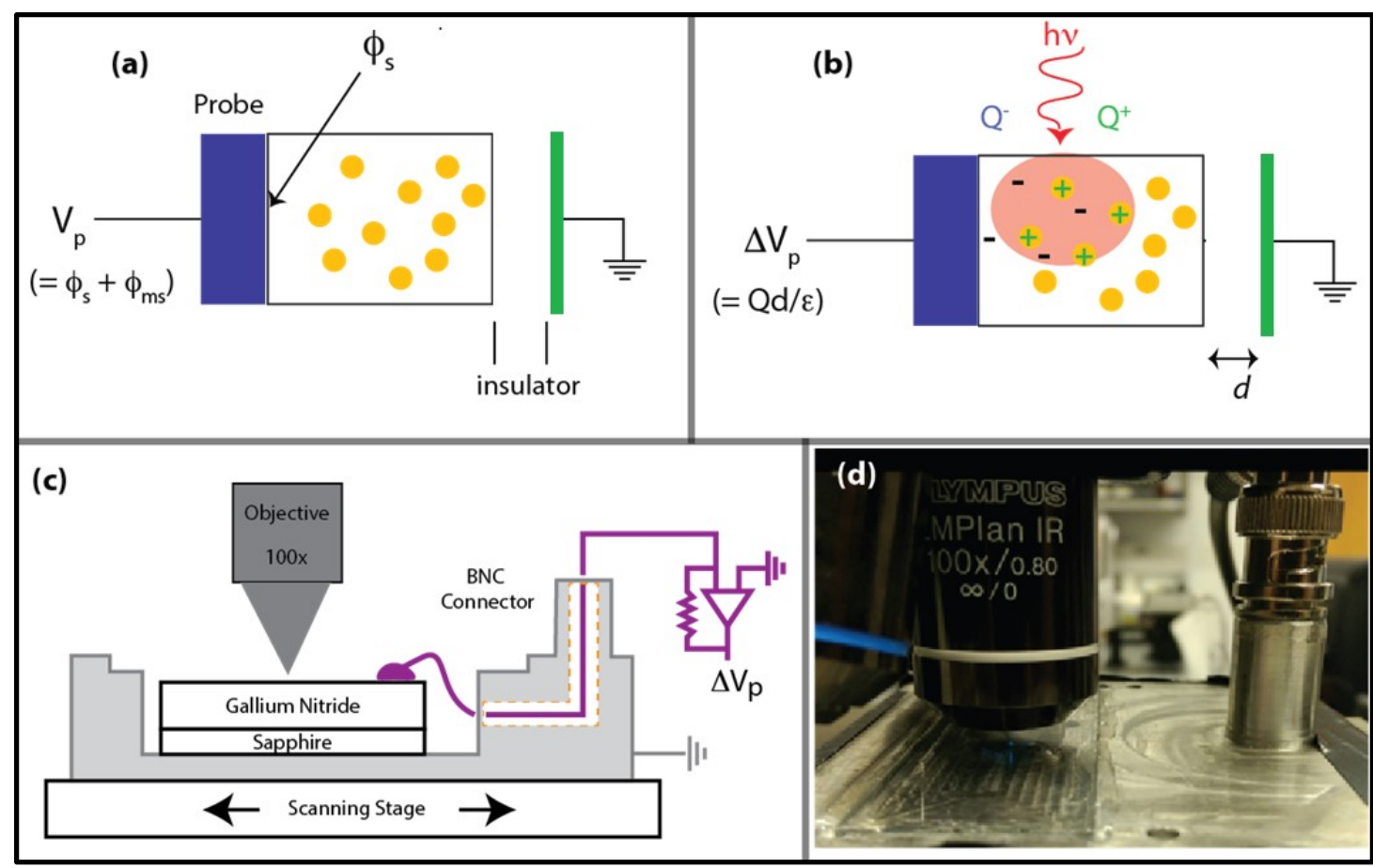

Figure 1.14: The scanning capacitive photocurrent technique used for STED electrical imaging. The voltage on a floating probe with (a) no illumination and (b) with illumination exciting charge from optically absorbing defect states. (c) Measurement set-up including sample holder for STED electrical imaging (d) Photo showing objective and sample holder.

material in the depletion beam will not generate a significant signal. This means that we can use a Gaussian excitation beam centered on the toroidal depletion beam for an effective spot size of $\sim 20 \mathrm{~nm}$, well below the diffraction limit. We can then mechanically chop the excitation beam to generate the AC signal within the $\sim 20 \mathrm{~nm}$ area. In Figure 1.14 , we see how the chopped beam creates a detectable capacitance from the induced charge separations within the material. In (a) we see that the material has an initial, base voltage $\left(V_{P}\right)$ that is determined by the work function of the sample and electrode $\left(\varphi_{\mathrm{ms}}\right)$ 
and a built-in potential due to any trapped charge in the sample $\left(\varphi_{\mathrm{s}}\right)$, such that $\mathrm{V}_{\mathrm{P}}=$ $\varphi_{\mathrm{ms}}+\varphi_{\mathrm{s}}$. With the incident light, seen in (b), we have an induced potential difference, $\Delta \mathrm{V}_{\mathrm{P}}=\mathrm{Qd} / \varepsilon$, where $Q$ is the total charge generated per area and $d$ is the distance the charges separated. With the pulsed light source, the rapid changes in potential is measured as an AC current. In Figure 1.14 (c), we have the layout of the scanning-CPC, and in (d) we have a photo of the scanning-CPC technique in action. This signal goes through a lock-in and amplifier and finally processed into an image, as is typical in optical/fluorescence based microscopy. 


\section{CHAPTER 2 FIXED-POINT CAPACITIVE PHOTOCURRENT TECHNIQUES AND MEASUREMENT}

\subsection{Systems and designs}

The core of early fixed-point CPC measurements is the Optical Parametric Amplifier (OPA), was used for much the early CPC work, and for preliminary work for the materials used in the CPC-STED system. The OPA is a tunable pulse laser that provides wavelengths with $120 \mathrm{fs}$ pulses, a $1 \mathrm{kHz}$ repetition rate, and a range from $400 \mathrm{~nm}$ to $3 \mu \mathrm{m}$. This broad wavelength range paired with a high repetition rate allows for high signal to noise ratio and broad spectrums of a sample's CPC response. In the following subsections, we will discuss the OPA and the CPC technique in greater detail.

\subsubsection{OPA}

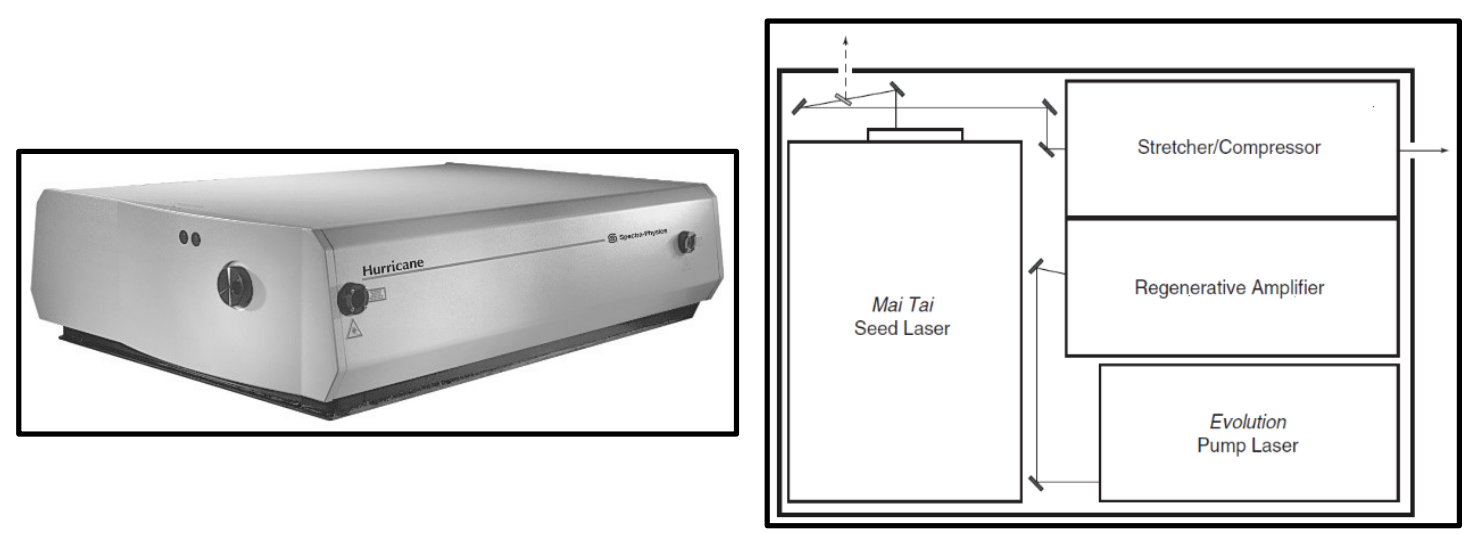

Figure 2.1: Hurricane system external and internal layout. [5]

The base of the OPA is its light source, a Spectra-Physics Hurricane laser. Since the $\mathrm{CPC}$ measurement is an $\mathrm{AC}$ measurement, the narrow pulse and rapid repetition rate match the requirements of this measurement. 
This beam profile results from the seed beam passing through a Ti:sapphire amplifier rod that is excited by the Evolution pump beam. The stretcher/compressor allows for this resulting beam to amplify with multiple passes, without exceeding the optical limits of the internal components. Figure 2.2 shows how stretching and compressing results in a final, high-powered pulse.

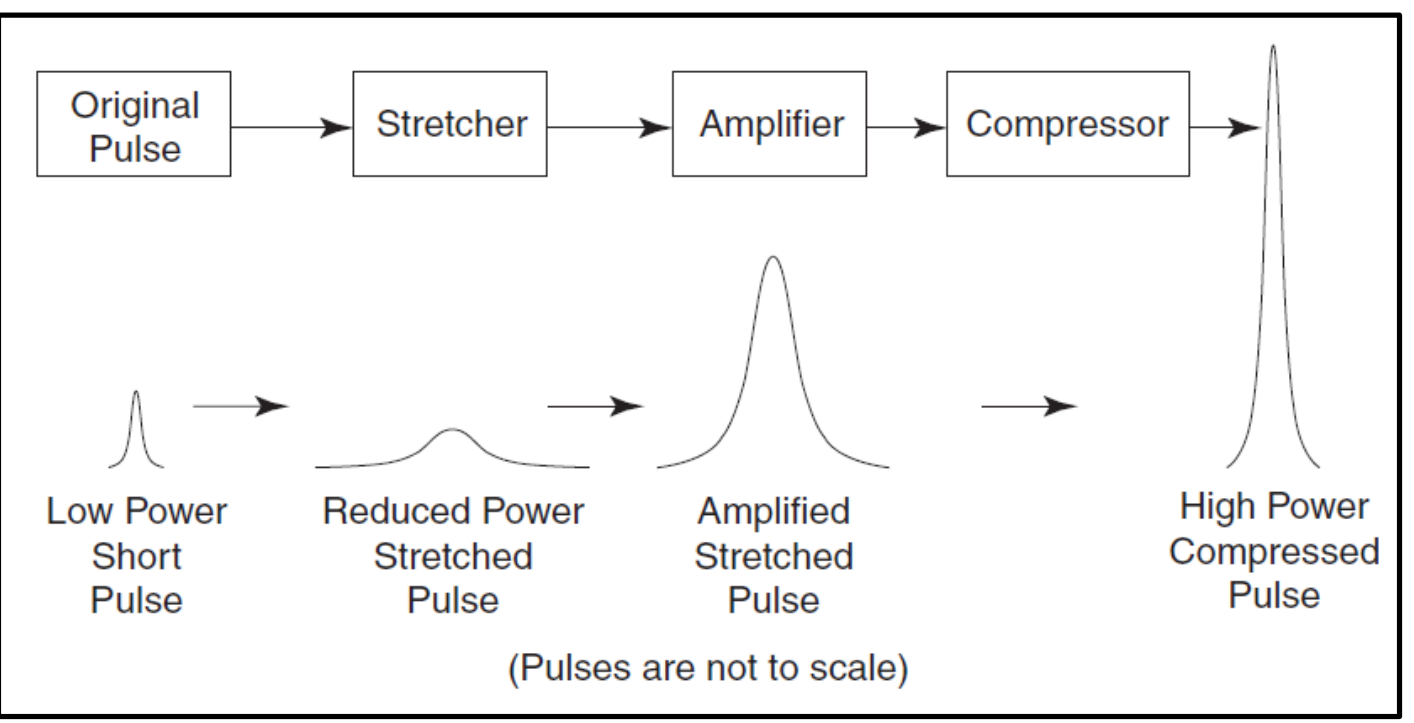

Figure 2.2: Stretching and compressing to prevent damage to optics with high power final output. [5]

\subsubsection{OPA Theory}

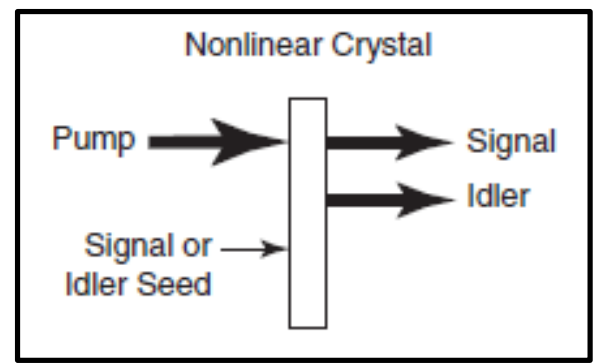

Figure 2.3: OPA function diagram. [4]

Optical parametric generation (OPA) describes the process where an input beam of light with frequency $\omega_{\mathrm{p}}$ passes through a nonlinear medium. This nonlinear medium provides output a signal frequency of $\omega_{\mathrm{s}}$ and an idler frequency of $\omega_{\mathrm{i}}$, where $\omega_{p}=\omega_{s}+$ $\omega_{i}$. In our system, the nonlinear component is a Beta Barium Borate (BBO). This 
configuration allows for second harmonic generation, sum-frequency, and differencefrequency mixings, as well as parametric generation, with a higher intensity pump beam and a lower intensity seed beam (signal or idler) to boost the signal and idler beams, as seen in Figure 2.3.

\subsubsection{OPA description and layout}

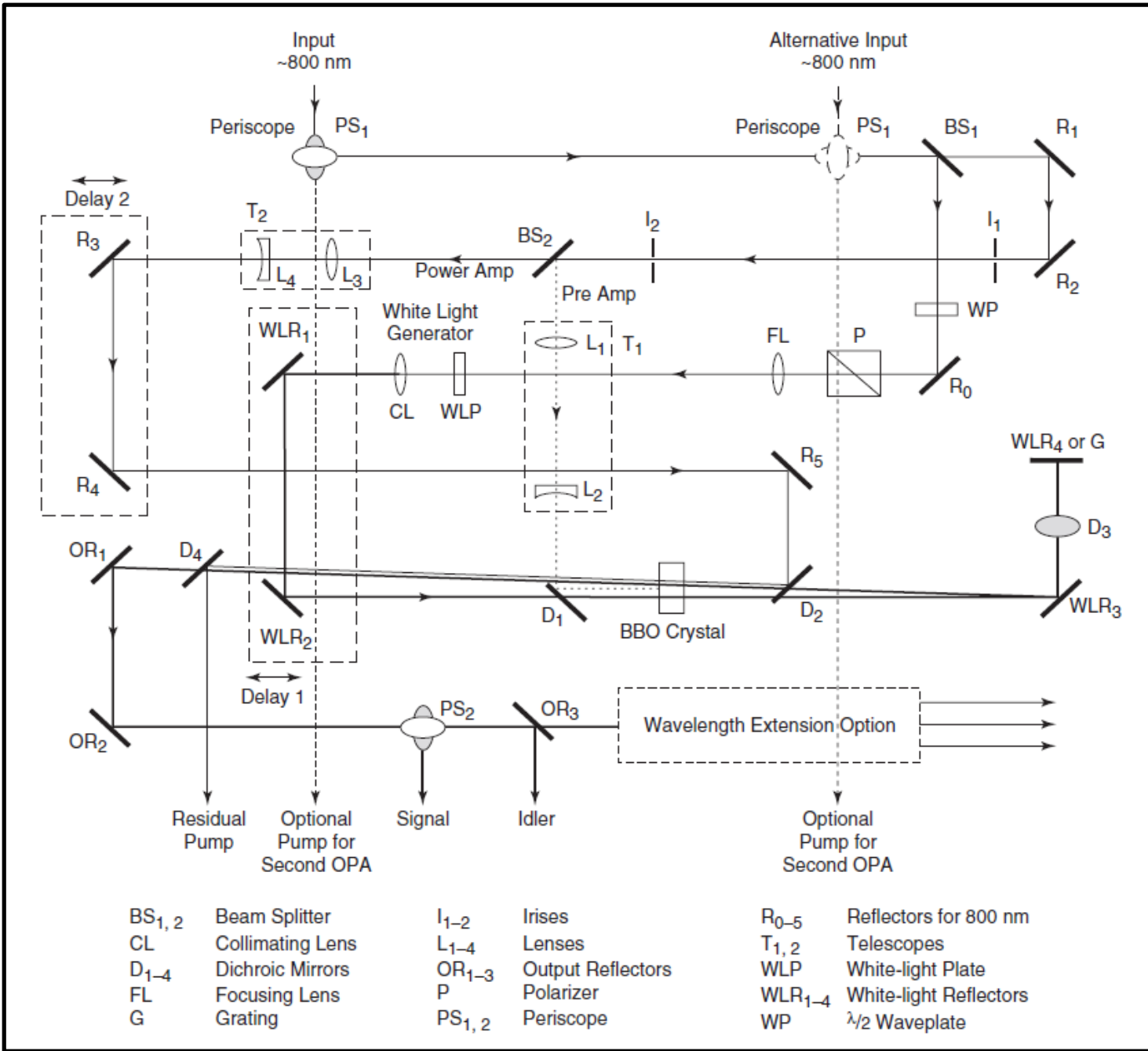

Figure 2.4: OPA configuration. [5]

To get the seed and pump beams separated at BS1 we have a 96/4 split of the beam there the $96 \%$ pumps the OPA and the remaining $<4 \%$ produces a white light continuum as the seed pulse of the OPA. A half waveplate and thin film control the 
beams power, allowing the beam to pass through the white light plate, generating the continuum. It is then re-collimated and passes through the variable delay stage Delay 1 and then sent through the BBO crystal. The amplified beam (96\% beam) splits into two parts, $\sim 15 \%$ of the beam follows the seed beam's first past through the BBO crystal as a pre-amplification beam. On the seed beams second pass through, the amplified idler beam passes through the $\mathrm{BBO}$ crystal with the remainder of the (power) amplified beam to achieve a final output. A BBO crystal controls the final output wavelength(s) by selecting its phase-matching angle, adjusting the Delay steps provides optimization of the final output beam's profile and power. We use further BBO crystals to further extend the wavelength regions, as seen in Figure 2.5 and Figure 2.6.
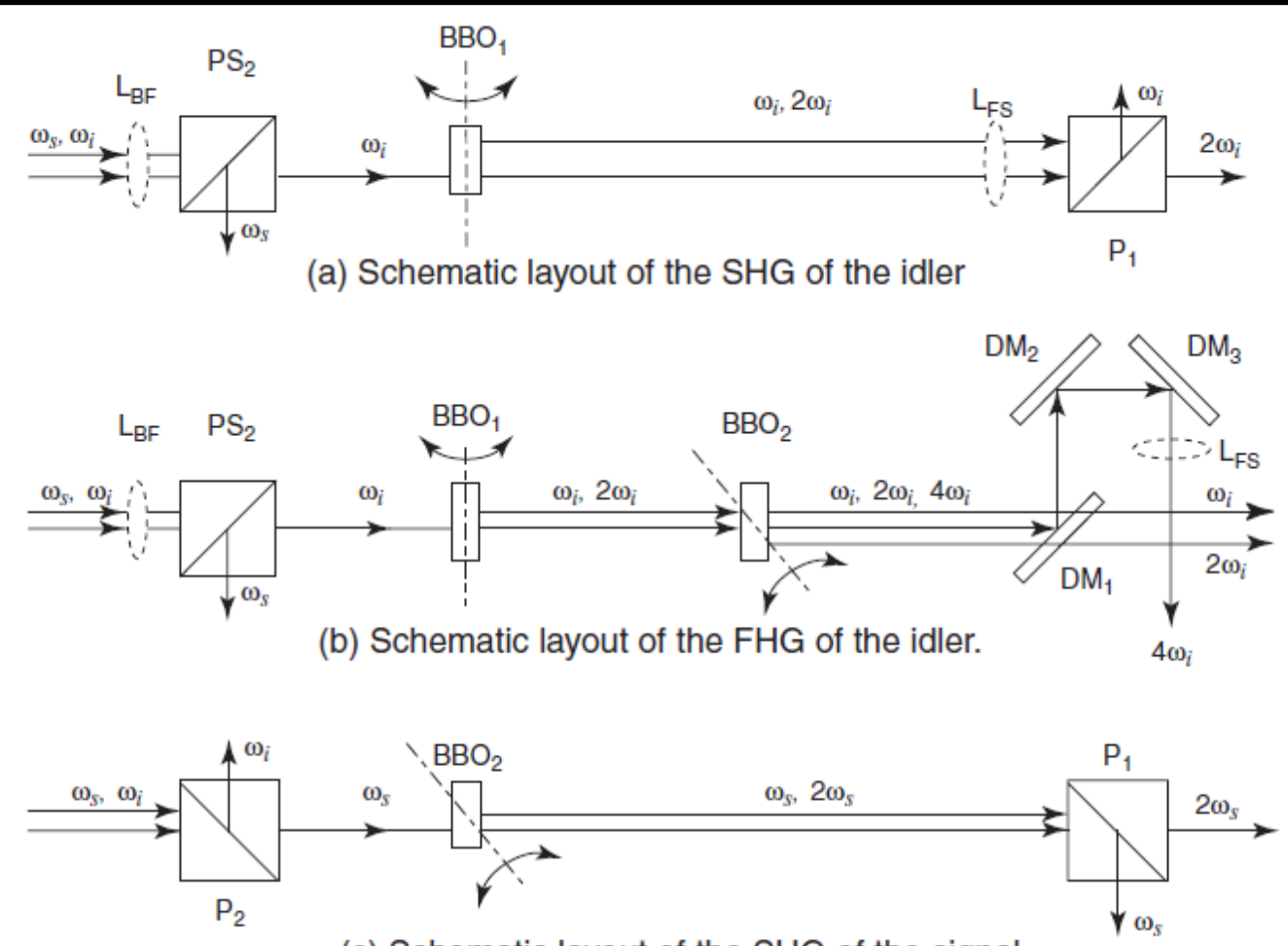

(c) Schematic layout of the SHG of the signal.

$\begin{array}{llll}\mathrm{BBO}_{1,2}: & \text { BBO Crystal } & \mathrm{L}_{\mathrm{FS}}: & \text { Fused Silica Lens } \\ \mathrm{PS}_{2}: & \text { Periscope 2 } & \mathrm{P}_{1,2}: & \text { Polarizers } \\ \mathrm{L}_{\mathrm{BF}}: & \text { BaF2 Lens } & \mathrm{KG}-3: & \text { Filter }\end{array}$

Figure 2.5: Configuration of OPA for wavelength extension. [4] 


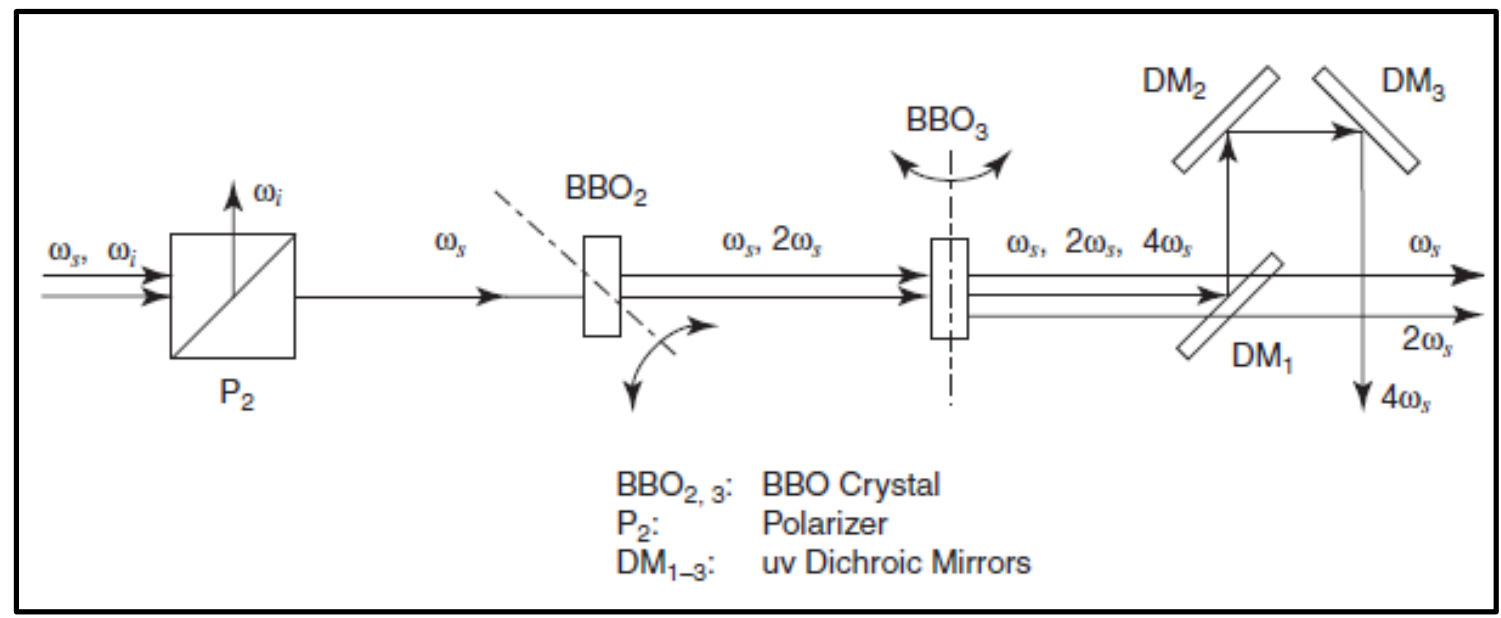

Figure 2.6: Configuration for FHG of the signal. [4]

\subsubsection{Continuum and AOTF}

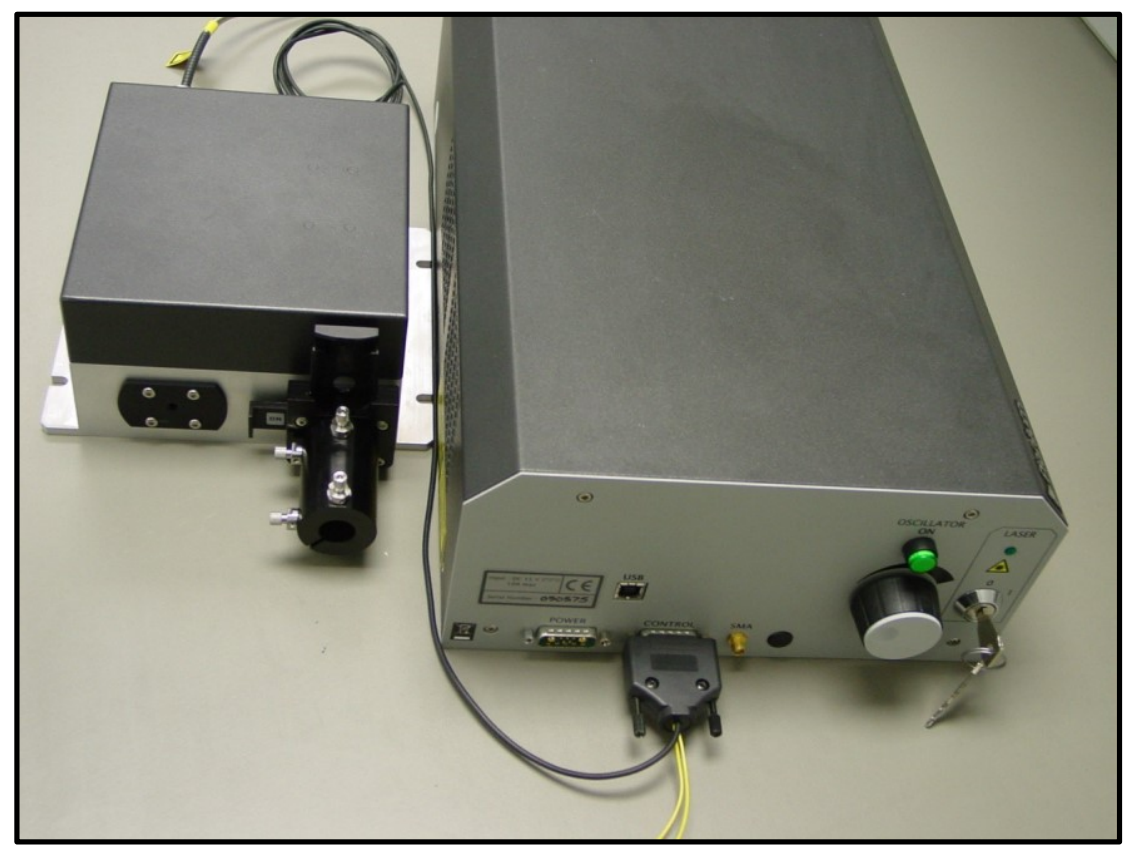

Figure 2.7: Fianium Supercontinuum with AOTF

The Fianium supercontinuum paired with AOTF (Acousto-Optic Tunable Filters) provided light from $400 \mathrm{~nm}$ to $650 \mathrm{~nm}$ in an easily controlled system. The AOTF allows for much quicker spectrum analysis when compared to the OPA system due to the ease of configurability. This system also allows for fiber mounted configuration, allowing for 
great control over beam deliver. In our later setups, this change allowed for use to change laser systems and light sources quickly when doing data collections.

\subsubsection{Continuum/AOTF Theory}

The backbone of this system is the supercontinuum. The Fianium Supercontinuum has three main components: a master source, a high-power amplifier, and a high nonlinearity supercontinuum generator. The master source is a passively mode locked fiber laser with a core-pumped Yb-doped fiber. This system has a fixed repetition rate and high pulse-to-pulse stability. A high power, multi-emitter laser diode pumps a double-clad Yb-doped fiber make the amplifying component of the system. The amplifier is the component that determines the final output power. The knob on the front is a tenturn potentiometer which adjusts the amplifier's power. Finally, the supercontinuum generator in this system is a highly-nonlinear optical fiber. With the source and amplifier generating high pulse peak power, the supercontinuum generator produces a spectrum from $450 \mathrm{~nm}$ to $1750 \mathrm{~nm}$. The output of the supercontinuum goes into the AOTF which has a visible range of $450 \mathrm{~nm}$ to $650 \mathrm{~nm}$. The AOTF functions by generating an acoustic wave through a crystal oscillated at frequencies that produce the desired wavelength from

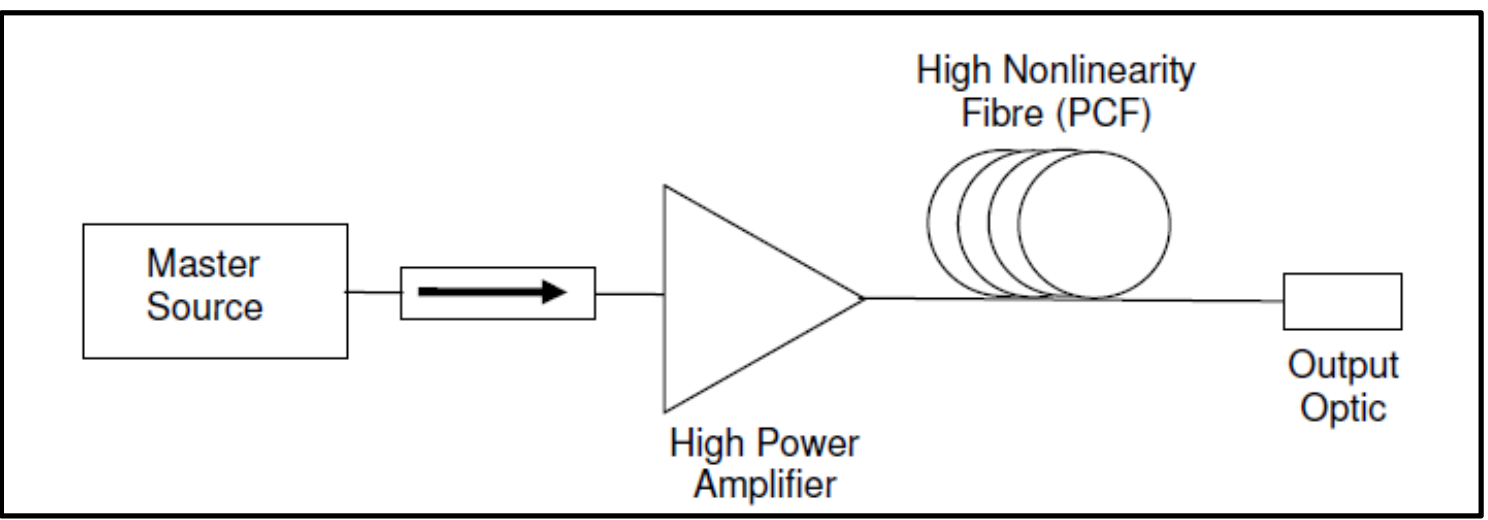

Figure 2.8: Functional diagram of a supercontinuum fiber light source [3] 
the broadband generated by the supercontinuum. This is all controlled automatically by the proprietary software.

\subsubsection{Continuum description}

The design of the Continuum system allows for easy deployment into our system. The output of the AOFT is collimated and focused into an optical fiber. The output of the fiber is placed in the preexisting fiber mount of the microscope setup. This ease of light source replacement means that we can focus the beam onto samples easily and accurately. With the fiber coupled at the output of the AOTF adjusting the wavelength is nearly trivial as shown in Figure 2.9. The software also provides the option for programing scanning parameters, setting wavelength, power, and dwell time.

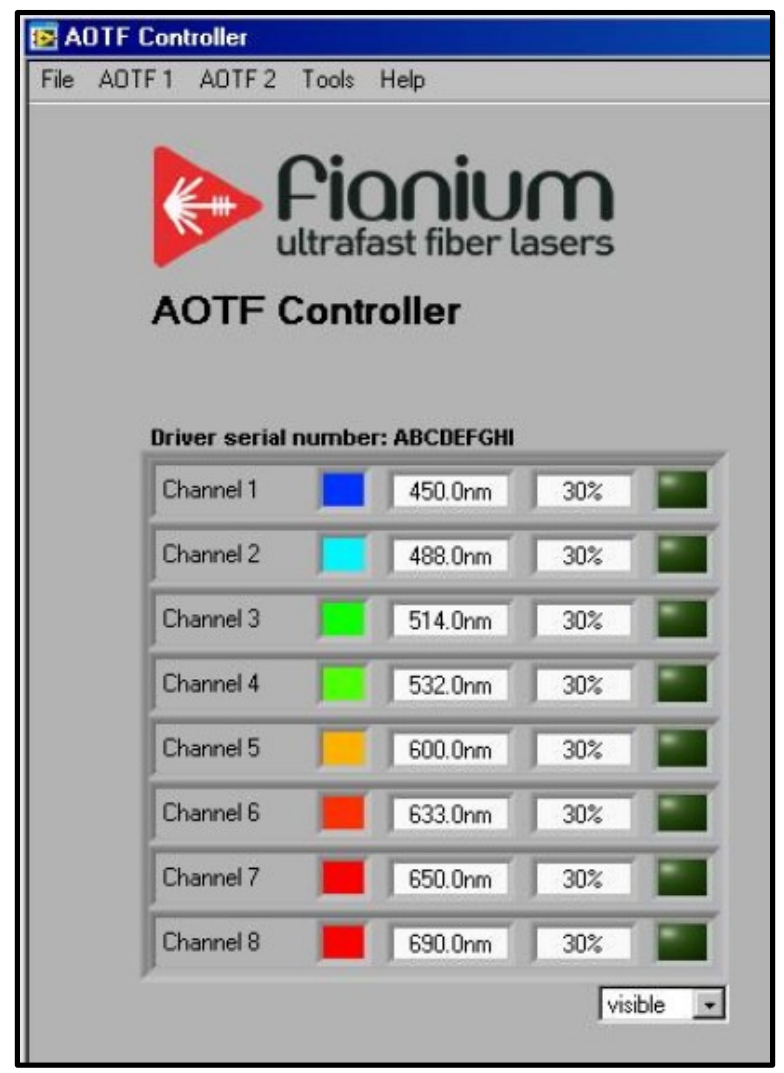

Figure 2.9: AOTF user control front panel. [15, 16] 


\subsection{Previous CPC investigations}

This section will discuss the development and uses of the CPC technique. First, the section will discuss the prior work done using this technique and the contributions provided. Next, we will discuss the work that I have been involved with personally and what contributions that work has provided.

\subsubsection{Carbon nanotubes}

Our group first used CPC to study carbon nanotubes. In 2005, our group published a pair of papers studying single and double walled nanotubes. The CPC is well suited to these materials because it provides a high signal to noise ratio as well as detecting both semiconductor and metallic transitions [21]. Figure 2.10 we have the schematic for signal detection (a) as well as a diagram showing the standard method for photocurrent measurements (b) and the CPC method (c). The CPC method lacks a dark current, allowing for detection of the metallic transitions in the nanotubes. Validating this

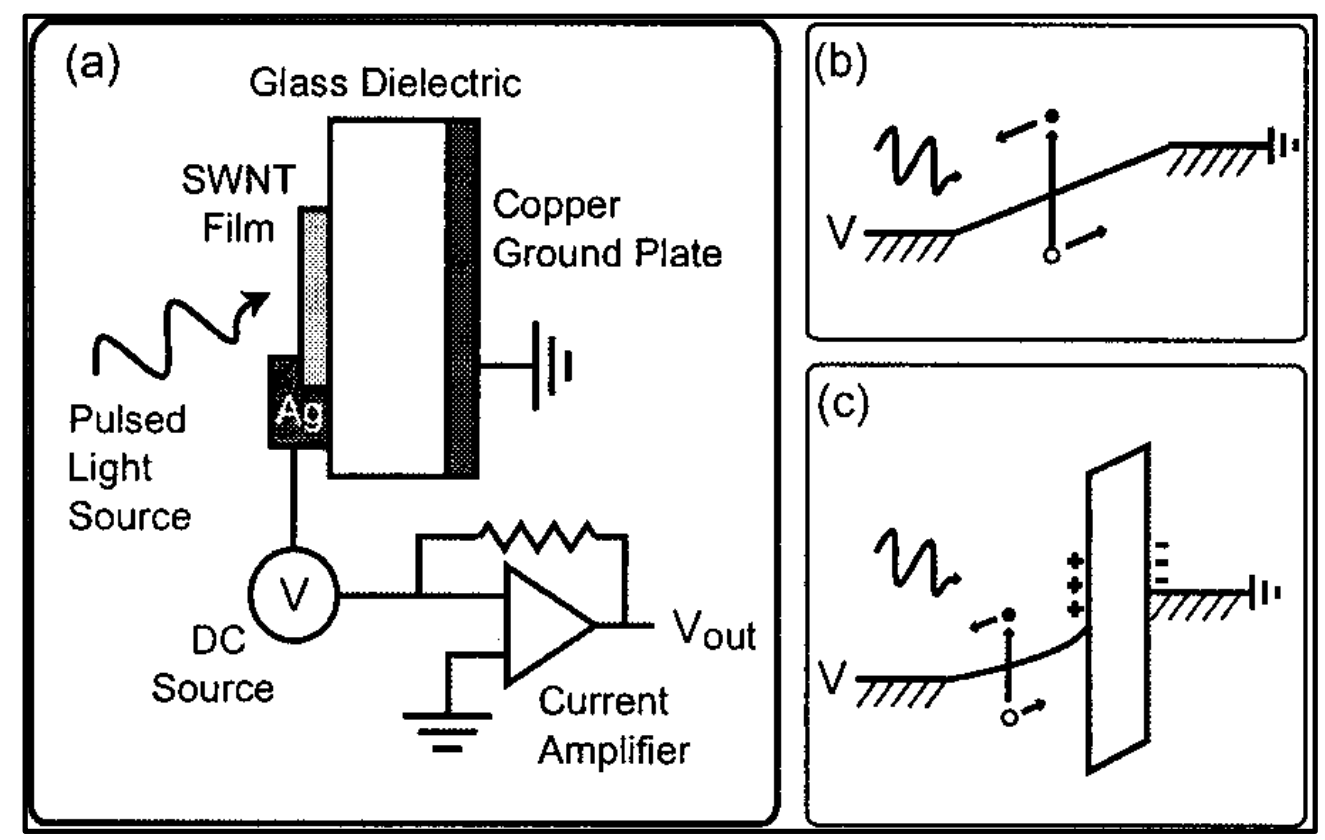

Figure 2.10: Single wall nanotube CPC investigation. [21] 
method involves comparing the CPC results (displacement current) with the nanotube's absorbance, as seen in Figure 2.11.

Using this technique with double wall carbon nanotubes (DWNT) also provides unique information. Using the CPC technique with Raman spectroscopy provides information on the how the curvature of the inner tube in a DWNT effects the distribution

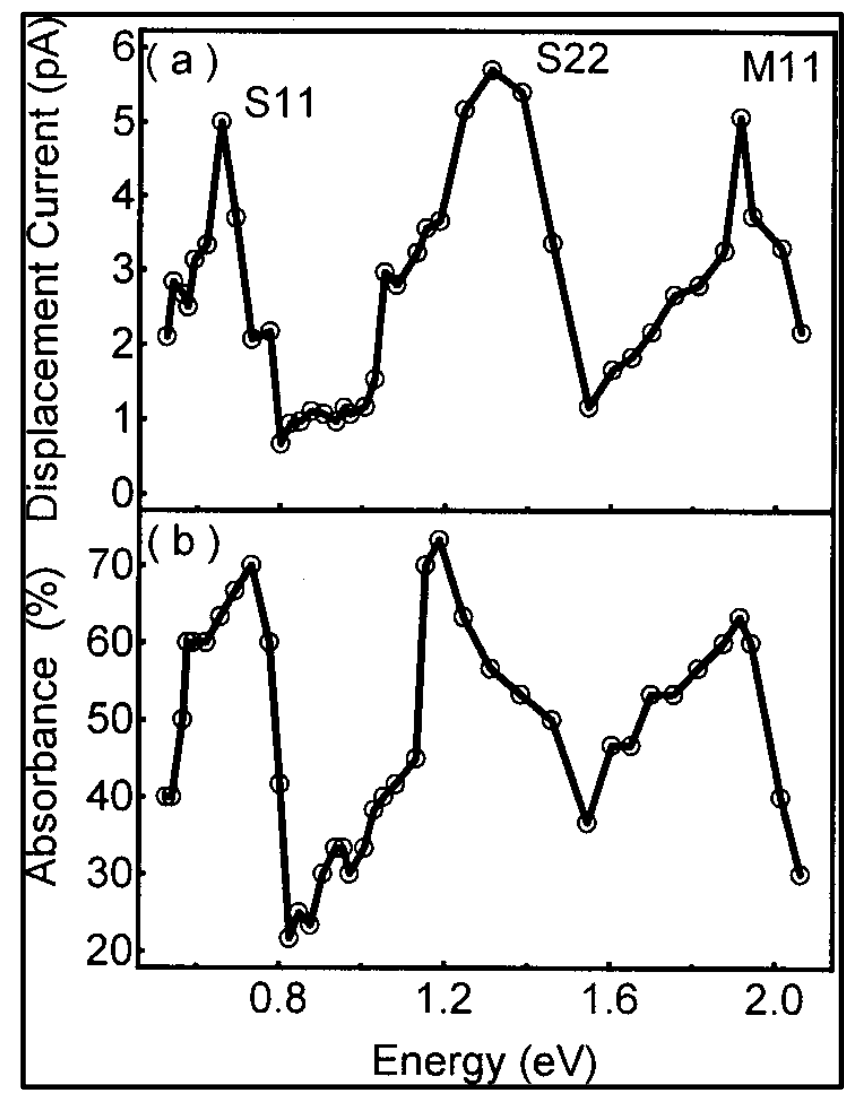

Figure 2.11: Comparison of SWNT CPC to absorbance. [21]

of the nanotubes electron states [28]. The Raman spectroscopy provides the diameter of the inner and outer shell and correlates to the observed features of the electron energy spectrum.

The CPC technique then offered the opportunity to learn more about the states within SWNT. Applying a DC bias across the SWNT sample allows for distinguishing between free-carrier and bound-excitonic transitions. This led to a paper showing the first 
evidence for excitonic states in metallic nanotubes [29]. Furthermore, the CPC technique is also useful in determining the effect of a perpendicular electric field on SWNT's excitonic and free carrier transitions. CPC provides evidence of field-induced mixing of excitonic and free carrier states in carbon nanotubes [30].

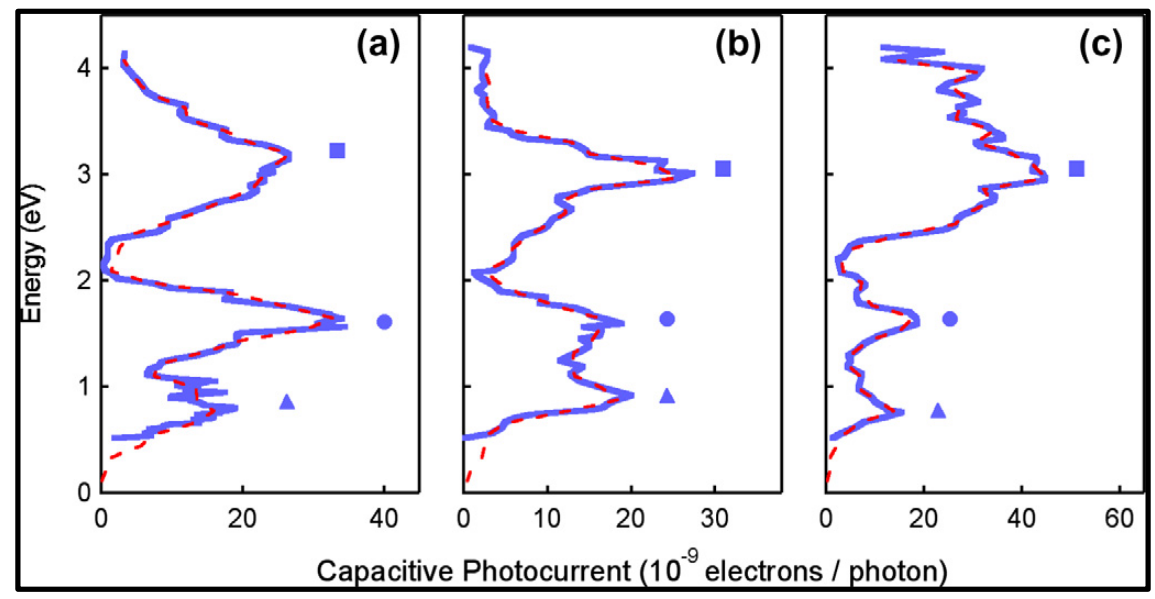

Figure 2.12: CPC spectra of three graphene-oxide samples with peaks at $0.7 \mathrm{eV}, 1.6 \mathrm{eV}$, and $3.2 \mathrm{eV}$. [14]

CPC also provides information on the effects of strain on SWNT. CPC shows that strain causes a shift in the energy of the ground state free electron transition as well as that of higher order excitonic transitions. CPC showed how it could be possible to use SWNT as high gauge factor optical strain sensors [31]. 


\subsubsection{Graphene oxide}

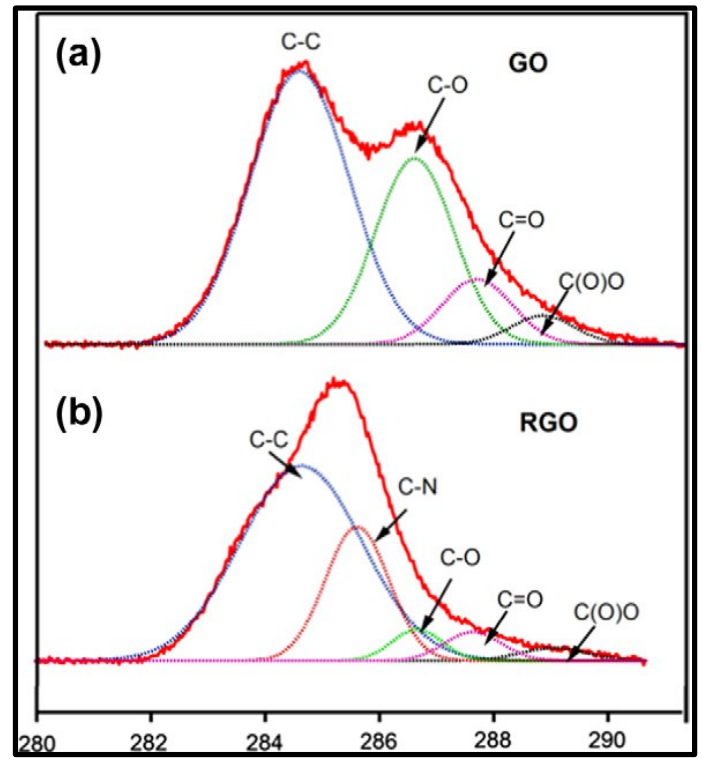

Figure 2.13: XPS of graphene-oxide (GO) and reduced graphene-oxide (RGO) [14]

CPC provides information on the electronics states of graphene-oxide and reduced graphene-oxide. With CPC, we can directly probe the density of states of the grapheneoxide. With this technique, we see three additional peaks in the density of states for the graphene-oxide, as seen in Figure 2.12. These additional peaks seen in the CPC are near the $\pi / \pi^{*}$ peaks, near the Fermi energy. This result is then corroborated by comparing the CPC peaks with an $\mathrm{x}$-ray photoelectron spectra which showing the various oxygen groups when the spectra is shown with its de-convoluted components, as seen in Figure 2.13. 


\subsubsection{BHJ solar cells}

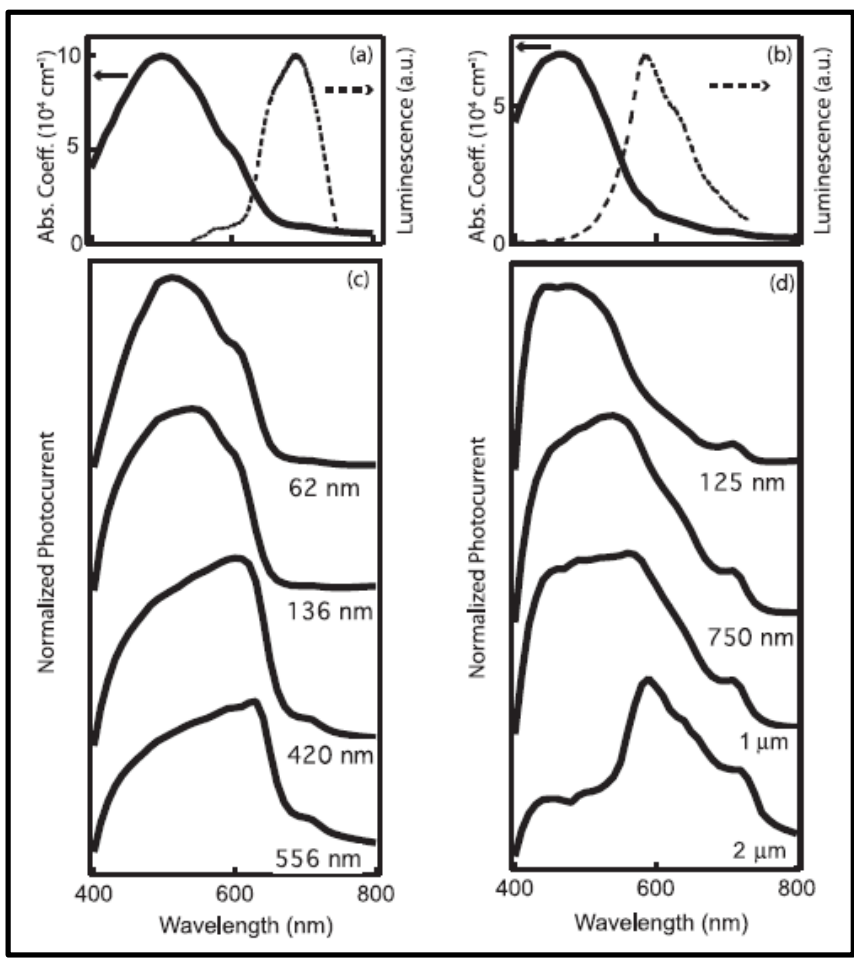

Figure 2.14: Absorbance and luminescence for P3HT (a) and $M D M O O P P V(b)$ and the CPC for each respectively $(c, d)$. [20]

With rising interest in both organic and bulk heterojunction solar cells, the CPC technique provides insights into the electronic function of $\mathrm{P} 3 \mathrm{HT}$ and $\mathrm{MDMO}-\mathrm{PPV}$ as $\mathrm{BHJ}$ components. As the layers of both $\mathrm{BHJ}$ material thicken in the active layer, the $\mathrm{CPC}$ signal shifts red, as seen in Figure 2.14. We see that for thin active layers the maximum CPC signal correlated well with the absorbance maximum for the range. Yet, with thicker active layers the maximum $\mathrm{CPC}$ shifts to longer wavelengths, suggesting that absorbance is no longer the limiting factor, but the limiting factor becomes the recombination process or electron diffusion. By correlating the absorption coefficient with the CPC peak at each active layer thickness, we have details of carrier collection lengths as well as values for the percentage of carriers lost to surface combinations [20]. 


\subsection{Contemporary CPC investigations}

This section will cover the analysis done using the CPC technique that I have done personally. They include the details of PCBM's possible polaronic states as shown with CPC. By understanding the fixed-point results of these samples, the twodimensional images are easier to interpret.

\subsubsection{PCBM}

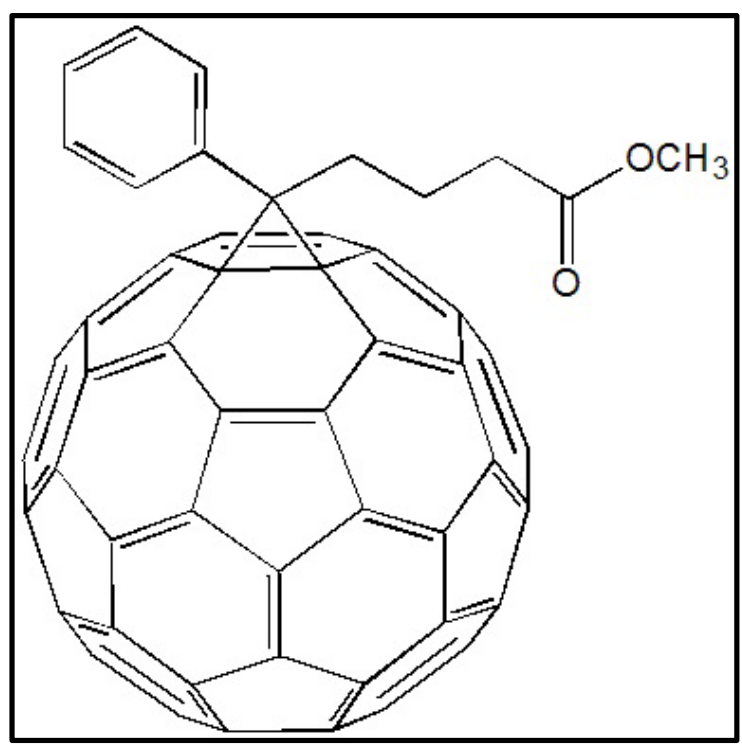

Figure 2.15: $P C_{60} B M[8]$

This section contains work utilizing the $\mathrm{CPC}$ technique with my direct involvement. This work led to two papers. One paper was an SPIE proceeding and the second was published in the Journal of Physical Chemistry C. PCBM, a fullerene derivative, is an electron acceptor in organic solar cells due to its high electron affinity and relatively strong absorbance in the visible region. 
The electron transfer distorts the fullerene lattice in the PCBM forming a polaronic state. With standard optical absorbance measurements, these polaronic states are not detectable. Identifying and understanding these states is essential to developing PCBM in solar cells since these characteristics determine the photo charge transport and collection. With CPC, we detect these polaronic state.

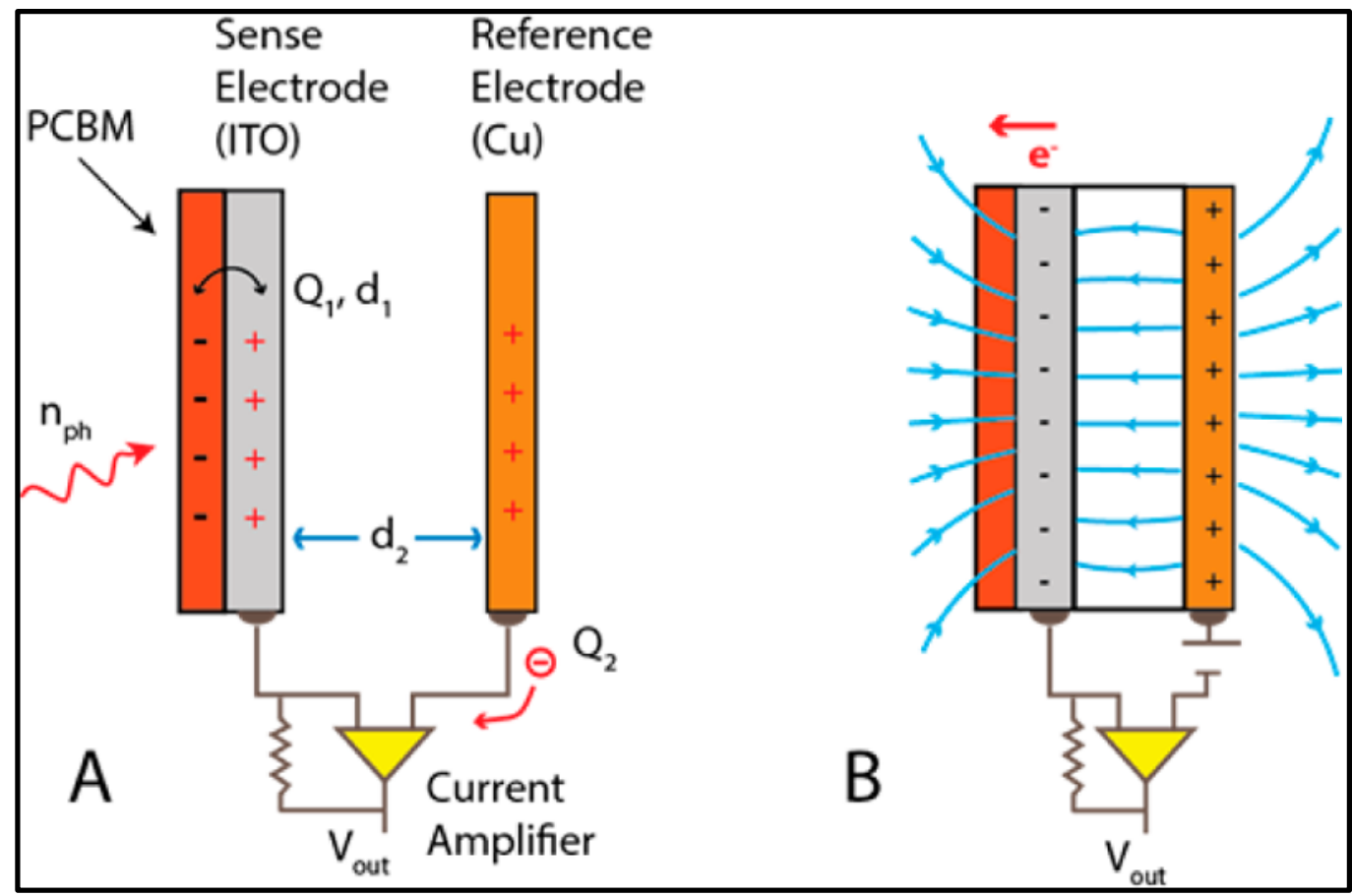

Figure 2.16: Collecting CPC signal of PCBM (a), with the effect of biasing (b) [2]

Figure 2.16 shows the diagram of CPC collection (a) as well as positive biasing (b) with respect to the copper block and ITO, enhancing the electron flow into the PCBM where a negative bias would block this flow. The PCBM is drop cast onto an ITO coated glass slide. We mount the sample onto a copper block, place inside a cryostat, and evacuate to HV/UHV conditions. Using the OPA system as the light source, we keep the laser power at a constant $5 \mathrm{~mW}$ for each wavelength used. With a photon pulse generating mobile charge of Q1, separated across a distance $\mathrm{d}$ 1, we have a potential raised across the ITO electrode given by, 


$$
V=\frac{Q_{1}}{C_{1}} \propto Q_{1} d_{1}
$$

Where $\mathrm{C}_{1}$ is the capacitance between PCBM and the ITO. To maintain equilibrium with the copper block, a small amount of charge is drawn through the current amplifier, where $\mathrm{d}_{2}$ is the distance between the ITO and the copper block.

$$
\Delta Q \approx Q \frac{d_{1}}{d_{2}}=\alpha q n_{p h} \frac{d_{1}}{d_{2}}
$$

Where $\mathrm{d}_{2}$ is a standard microscope slide thickness of $d_{2}=10^{-3} \mathrm{~m}$. With a $5 \mathrm{~mW}$ laser, we have $n_{p h} \sim 10^{16}$ photons $/ s$, and estimating a reasonable charge seperation distance of $d_{1}=10^{-9} \mathrm{~m}$, and knowing that our CPC measurements are the in pico-amp regieme, these values mean that we have a photon to charge efficiency of, $\alpha=0.1 \%$, as estimated.

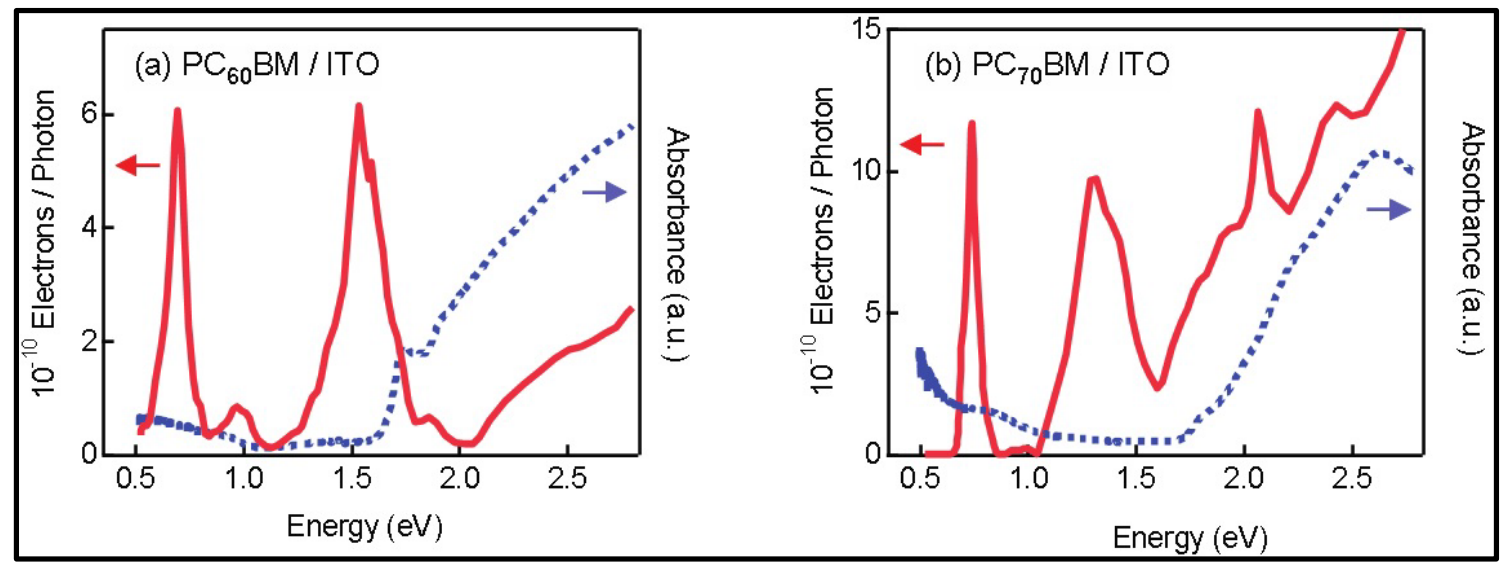

Figure 2.17: CPC vs Absorbance of $P C_{60} B M(a)$ and $P C_{70} B M(b)$ [8]

The $\mathrm{CPC}$ of $\mathrm{PC}_{60} \mathrm{BM}$ show features at $1.5 \mathrm{eV}$ and $0.7 \mathrm{eV}$ as well as an absorbance edge at $1.74 \mathrm{eV}$ which correstons to its $\mathrm{S} 1$ transitions. Similarly, the $\mathrm{PC}_{70} \mathrm{BM}$ has peaks at $1.3 \mathrm{eV}$ and $0.73 \mathrm{eV}$.

By depositing layers of $\mathrm{LiF}$ on the ITO prior to drop casting the PCBM, we create a tunnel barrier. In Figure 2.18, with increasing thicknesses of the LiF layer, we have a decrease in both the CPC values at the $1.5 \mathrm{eV}$ (b) and $0.7 \mathrm{eV}$ (c) as well as an overall 
decrease in the spectra (a). With the current decreasing exponentially, with respect to LiF thickness, we see the expected results of Fowler-Nordheim tunneling through this insulating layer. This result demonstrates that observing a CPC signal requires charge transfer.

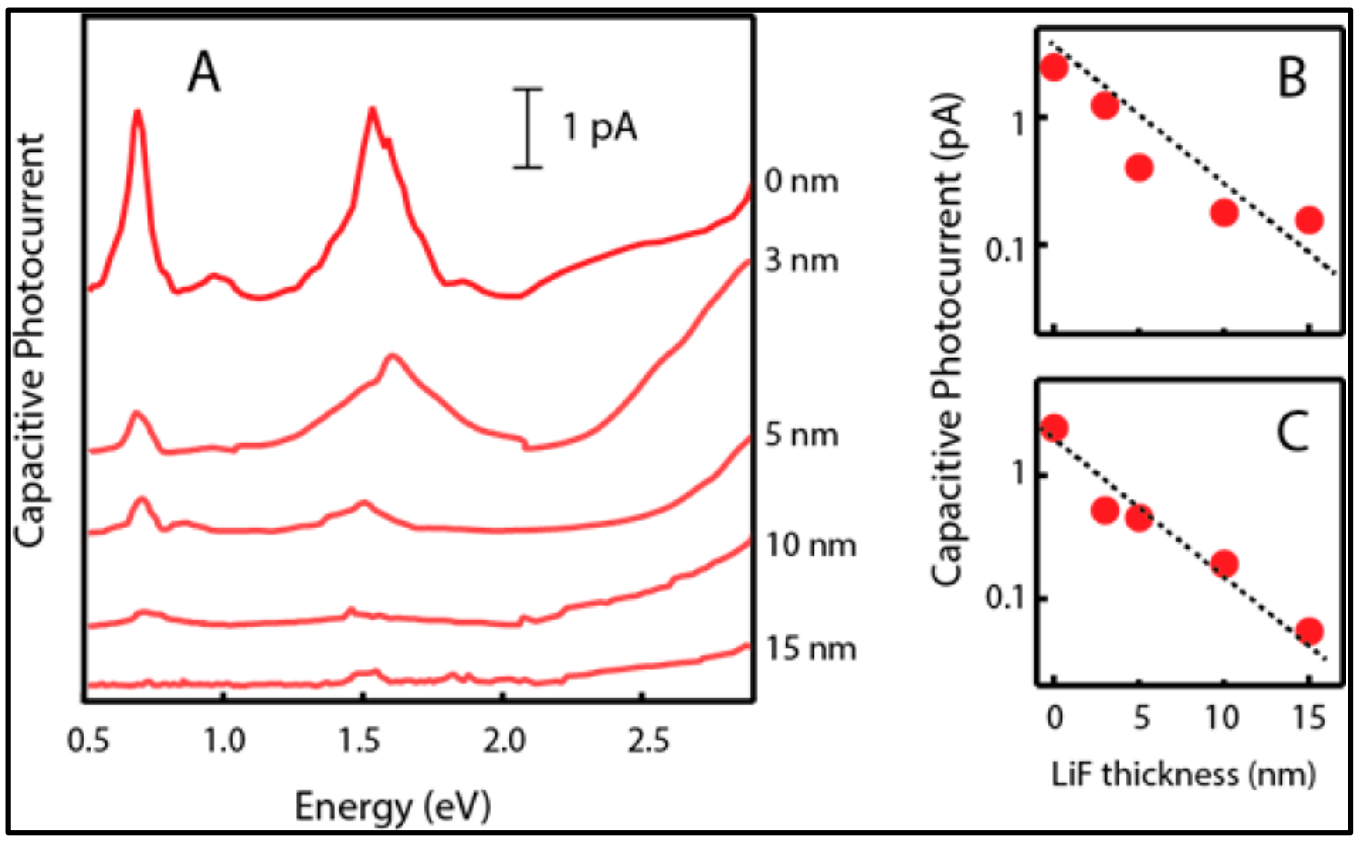

Figure 2.18: Effect of including insulating LiF layer. (a) Spectrum, (b) effect of increasing LiF thickness on CPC signal at $0.7 \mathrm{eV}$ (c) same at $1.5 \mathrm{eV} \mathrm{[2]}$

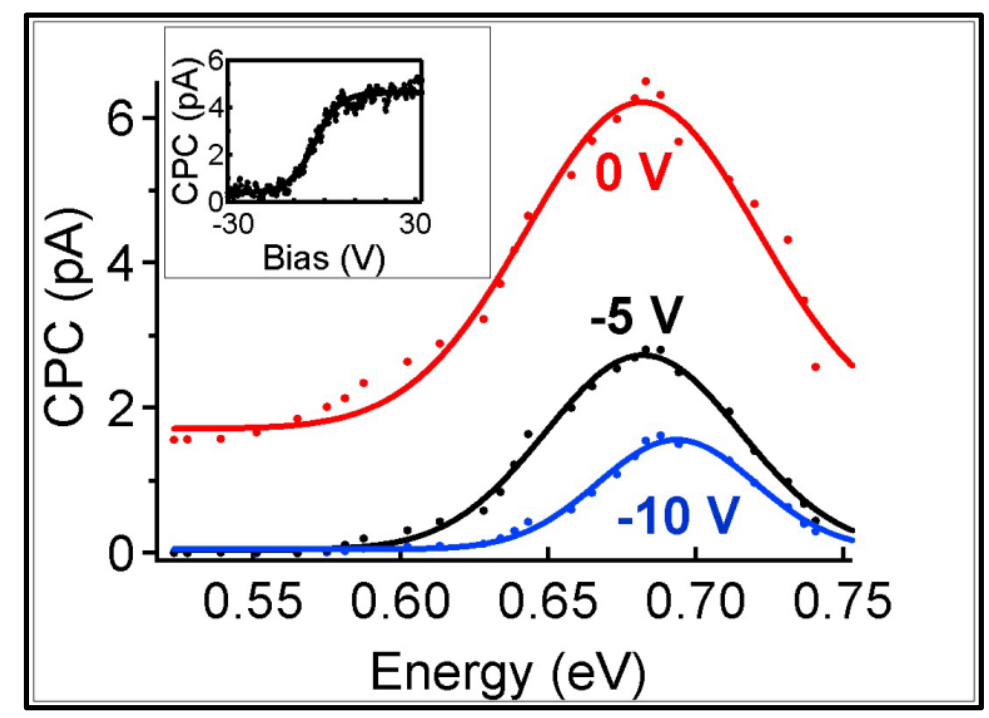

Figure 2.19: Biasing of PCBM at $0.7 \mathrm{eV}$

The next step requires determining the polarity of the charge transfer across the ITO/PCBM interface. Biasing the sample shows a dampening of the signal as the 
negative bias increases in magnitude. With the DC biasing, most of the voltage is lost to the insulator between the copper block and the ITO layer, but there is still a fringe electric field present at the ITO/PCBM interface. In Figure 2.19, we see the CPC of $\mathrm{PC}_{60} \mathrm{BM}$ at $0.7 \mathrm{eV}$ increasing negative biasing. Since increasing negative bias causes the signal to drop this indicates that the CPC signal is due to a negative charge transfer from the ITO into the $\mathrm{PC}_{60} \mathrm{BM}$. Inset in Figure 2.19 shows the CPC signal as a function of bias.

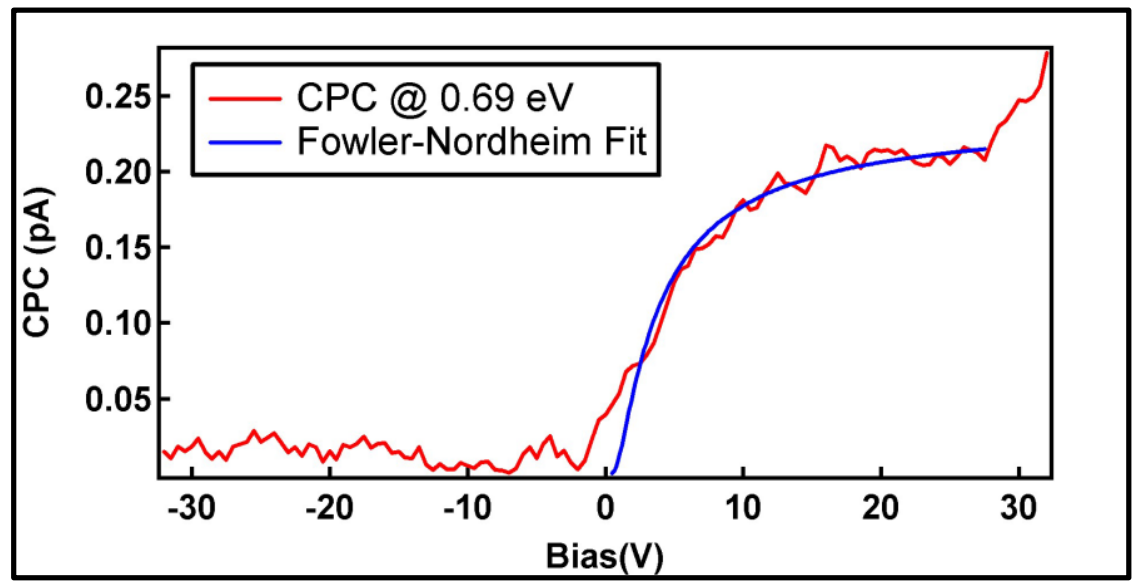

Figure 2.20: Subgap bias dependence with Fowler-Nordheim fit

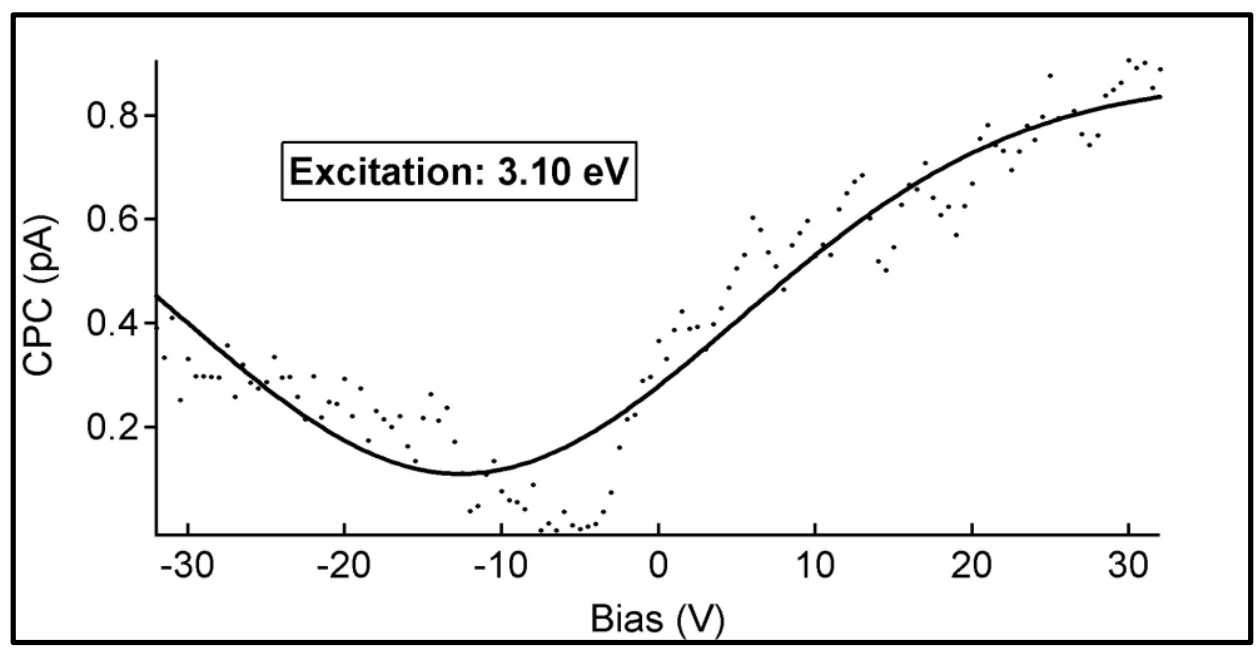

Figure 2.21: Above gap bias dependence

We see the same bias dependencies at the other below bandgap peak $(1.5 \mathrm{eV})$ or $\mathrm{PC}_{60} \mathrm{BM}$ where we have fit the Fowler-Nordheim tunneling prediction. This figure shows 
that there is a threshold bias at about $0 \mathrm{~V}$ where the signal turns on and eventually saturates with positive bias. This asymmetry of the bias dependency shows that to see the subgap peaks there must be negative charge injection in the PCBM. The positive bias saturations indicate that the number of photons limits the signal. For the above gap regime, we see a symmetrical bias dependence, as seen in Figure 2.21. This feature indicates that charge transfer is possible for both positive and negative because there are multiple neutral states above the band gap in PCBM, which do not require additional charges.

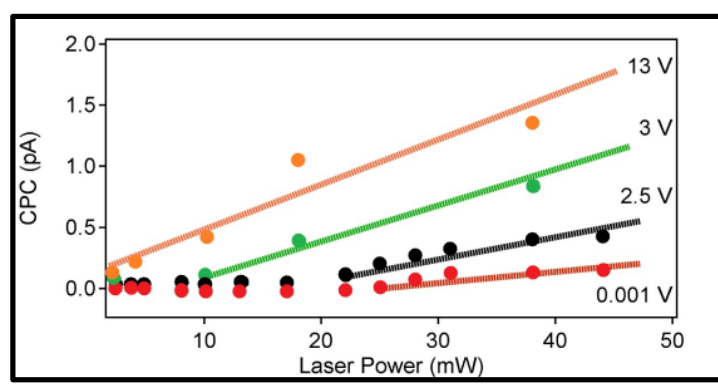

Figure 2.22: Bias vs laser power

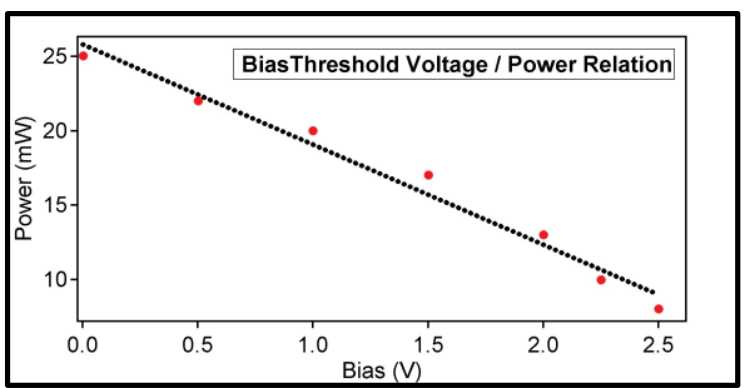

Figure 2.23: Laser power and biasing thresholds

We then evaluate the effects of the beam power with respect to the biasing, as seen in Figure 2.23. This figure also shows that there is a linear relationship between bias threshold voltage and laser power, as Figure 2.22 shows. This feature suggests that when either biasing or a laser power threshold is met, the process becomes linear implying a single-photon process resulting in a proposed model of the band diagram for the chargetransfer process, as seen in Figure 2.24. This model gives a potential barrier, $\mathrm{q} \varphi$, between the ITO and PCBM which is the energy difference between the ITO's Fermi energy and the lowest excited charged state in the PCBM. This value is equivalent to the energy required for negatively charging the PCBM. We have a potential drop across the PCBM 
bands with an electric field, $F$, at the ITO/PCBM interface. This model gives a tunneling current of,

$$
I=\alpha e^{-q \Phi / F}
$$

where, $\alpha$ is a proportionality constant; this relation provides the fit in Figure 2.20. With this good fit, it suggests that excitation at the polaronic transitions energies promotes both the charge transfer that causes the polaron to form and the optical excitation of the charge polaronic state [2]. There is also a need for thermalization as the threshold power dependence suggests. This model all matches well with the theoretical calculations of fullerene spectra using first-principle DFT for neutral, one added electron, and two added electron systems.

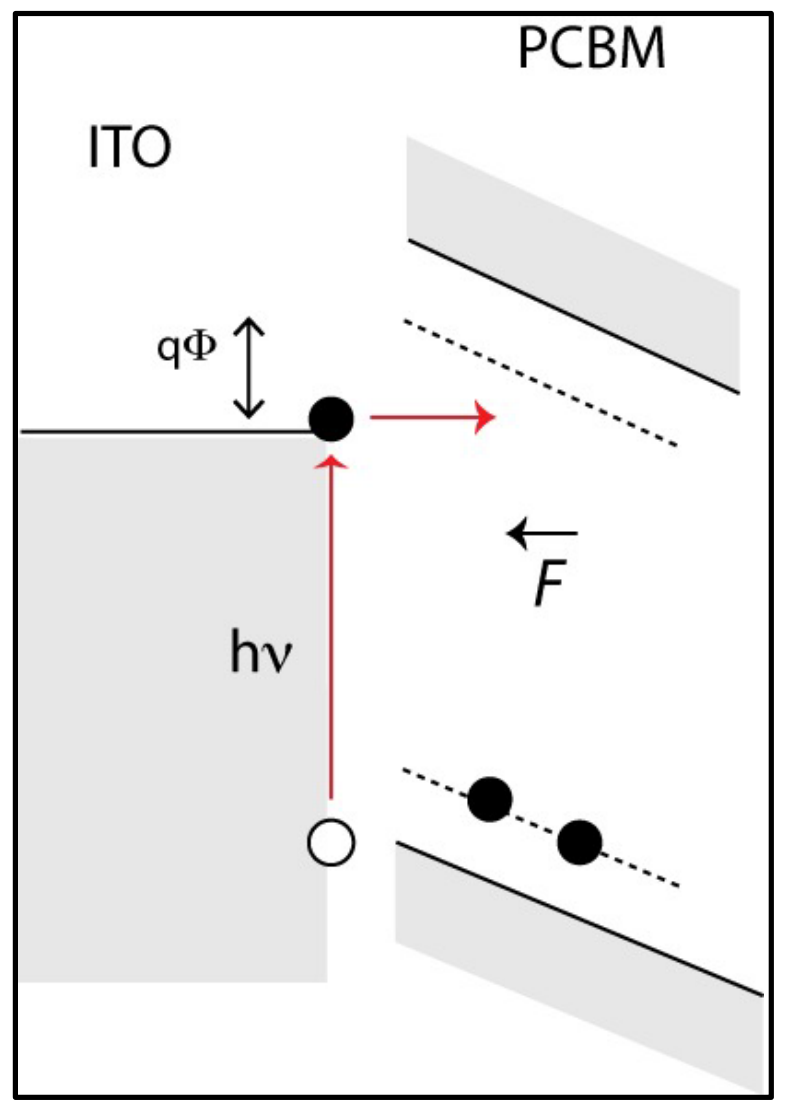

Figure 2.24: Band diagram for

ITO/PCBM charge-transfer process. 


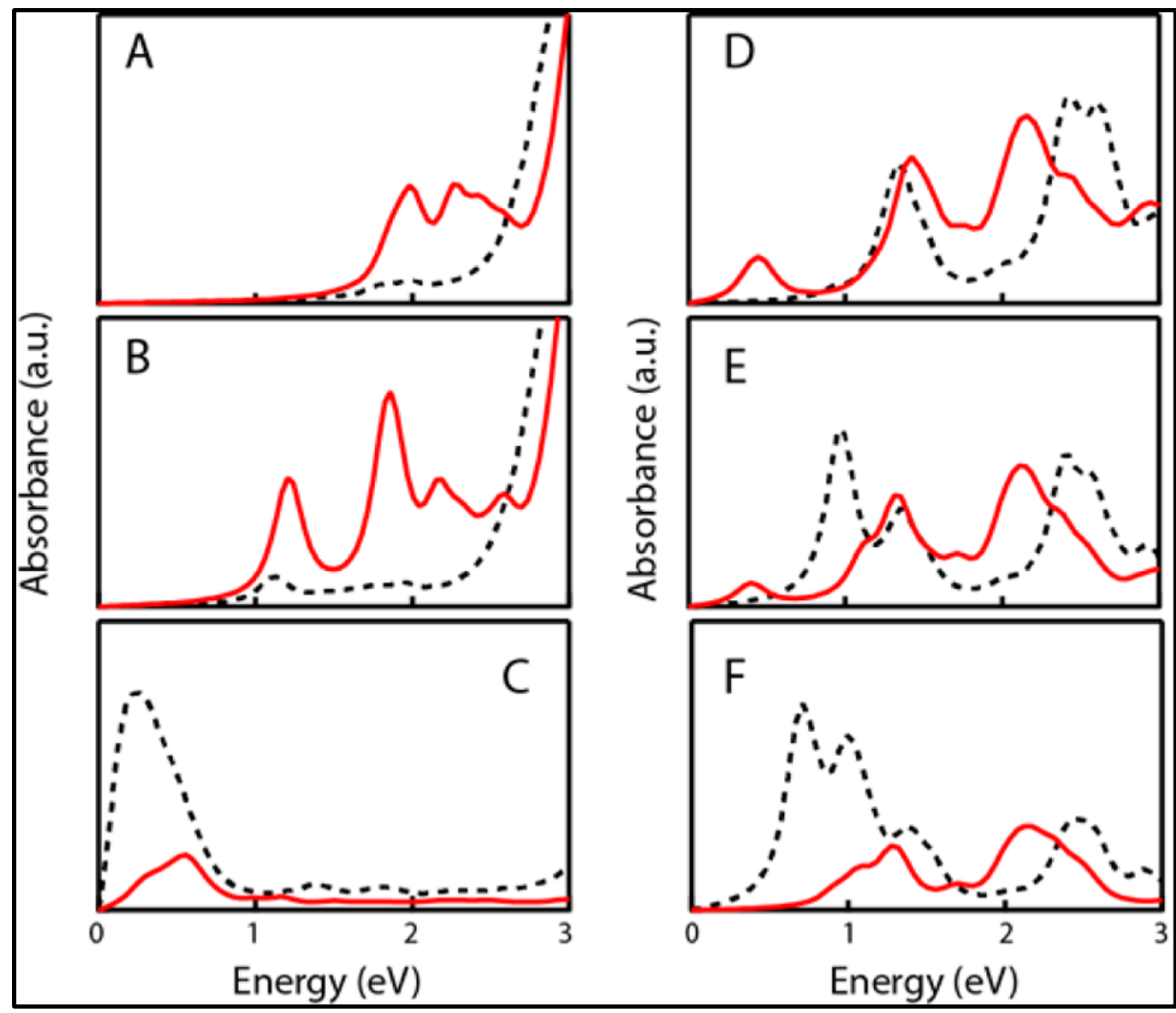

Figure 2.25: Calculated absorbance spectra for polymeric fullerene chains. The solid red line shows the ky polarized absorption and the dashed black line shows the $\mathrm{kx}$ polarized absorption. (A) Neutral C60 chain. (B) C60 chain with one added electron. (C) C60 chain with two added electrons. (D) Neutral C70 chain. (E) C70 chain with one added electron. (F) C70 chain with two added electrons. 


\section{CHAPTER 3 DEVELOPMENT OF THE SCANNING-CPC TECHNIQUE}

Since the CPC technique provides unique information and is usable in a wide variety of environments, it is well suited for a scanning system. Where fixed point CPC provides information about material properties, scanning-CPC can provide information about a material's properties in two dimensions. CPC provides information on defects, unique photo-excitation properties, as well as electrical nature. In Figure 3.1, we have the basic schematic for the scanning-CPC system.

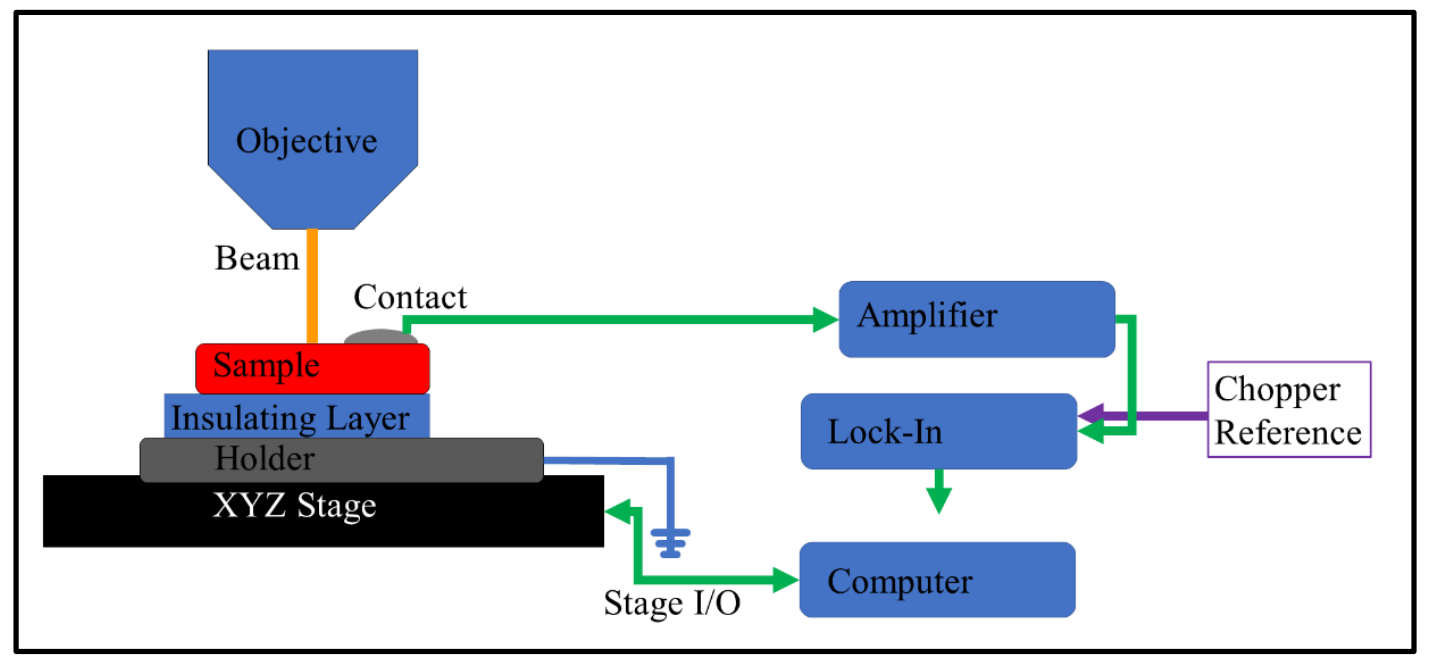

Figure 3.1: Basic design of scanning-CPC.

\subsection{Optical table layout}

With a final goal of scanning-CPC-STED, the system's layout accommodates the STED functionality as well as a single wavelength scanning functionality. The layout was designed and implemented by Dr. Sergio Mendes, Jafar Ghithan, and Dr. Rodrigo Wiederkehr. A removable phase plate enables this functionality, by moving the chopper to either the $2.54 \mathrm{eV}$ or $2.09 \mathrm{eV}$ beam path, we can scan either wavelength generating a 
CPC image. For full CPC-STED functionality, as seen in Figure 3.2, we have a $2.54 \mathrm{eV}$ beam generating an $\mathrm{AC}$ signal and a $2.09 \mathrm{eV}$ beam generating the depletion region.

The excitation beam for our system is $2.54 \mathrm{eV}$ generated by a Coherent Obis laser head with an output power range of $<5 \mathrm{~mW}$ to $\sim 60 \mathrm{~mW}$. A periscope (PS) positions the beam, coupling it to a single mode fiber (SMF) [P1-488PM-FC-5, Thorlabs], using a 40x objective with a numerical aperture of 0.65 [Newport] in the fiber launch (FM) [MAX350D, Thorlabs]. The output of the fiber is the collimated with a lens of focal length $18.4 \mathrm{~mm}(\mathrm{CL})$ [FC/APC, Thorlabs] and mechanically chopped [MC2000B, Thorlabs]. This chopping provides the AC current and the refence signal needed for the lock-in. A dichroic mirror (DM3) [ZT488rdc-UF2, Chroma] steers the chopped $2.54 \mathrm{eV}$ beam to pass through another dichroic mirror (DM4) [Z590sp-rdc, Chroma] into the microscope. The high-power depletion beam follows a similar path. An MPB Communications, Inc. laser head generates this beam with a power range of $150 \mathrm{~mW}$ to $1650 \mathrm{~mW}$. A fiber mount (FM2) couples the $2.09 \mathrm{eV}$ beam to a single mode fiber (SMF) where the output of the fiber is collimated (CL2). This collimated beam passes through a 50/50 split dichroic mirror (DM1) [WW11050-AR-A-SP, Thorlabs] where the reflected component is blocked, and then a vortex phase plate VPP-1 [RPC Photonic Inc., NY] generates the toroidal beam of the unblocked component. This toroidal beam is the core functionality of STED and CPC-STED microscopy. This toroidal $2.09 \mathrm{eV}$ beam then passes through another 50/50 split dichroic mirror (DM2). These mirrors mean that the final total power of the toroidal beam is $\sim 25 \%$ of the original power from the collimated fiber output. This $2.09 \mathrm{eV}$ beam is the steered by another dichroic mirror (DM4) into the microscope. When properly steered, at DM4 both beams will align such that they are 
collinear, this ensures that both beams will focus correctly through the objective [100x/0.80 LMPlanIR, Olympus] in the microscope [BX51M, Olympus] with their highest power and best profile, uniformly toroidal for the $2.09 \mathrm{eV}$ beam and Gaussian for the $2.54 \mathrm{eV}$ beam.

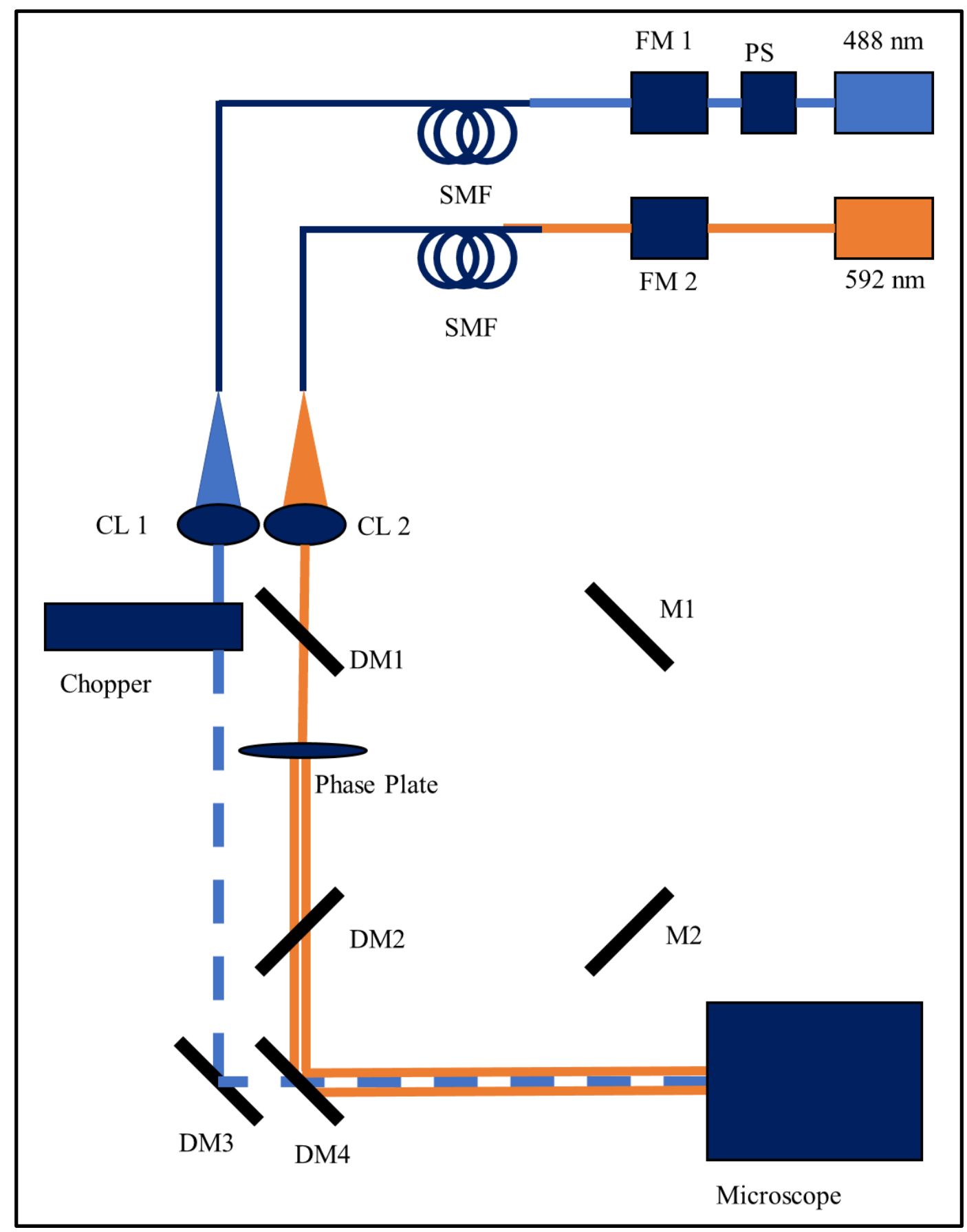

Figure 3.2: CPC-STED basic layout. 
Figure 3.3 shows an alternative layout for an alternative CPC-STED laser configuration. Since fluorescence and fluorescence depletion is not the goal of scanningCPC of CPC-STED but state depletion a dual $2.09 \mathrm{eV}$ setup is permissible. This design provides a higher power excitation beam as well as a simpler alignment. The first dichroic mirror (DM1) steers the reflected beam to a pair of high quality mirrors (M1, M2) and a chopper, providing the AC signal form the sample. A third configuration involves replacing the fiber at CL1 in Figure 3.2 with the output fiber from the Supercontinuum-AOTF system, providing a wider range of excitation wavelengths at the cost of beam power.

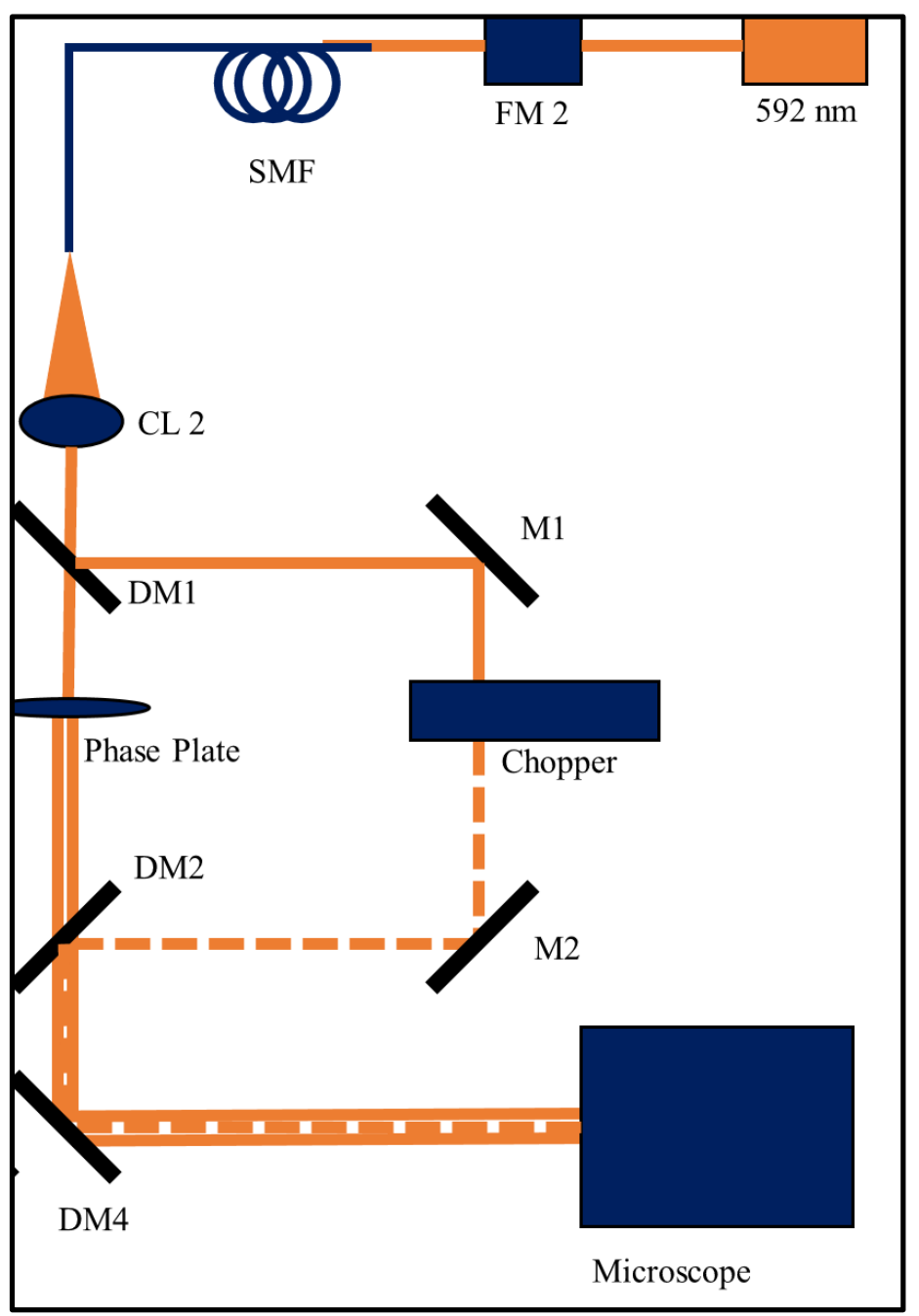

Figure 3.3: CPC-STED with dual 2.09eV beams 


\subsection{Holder design}

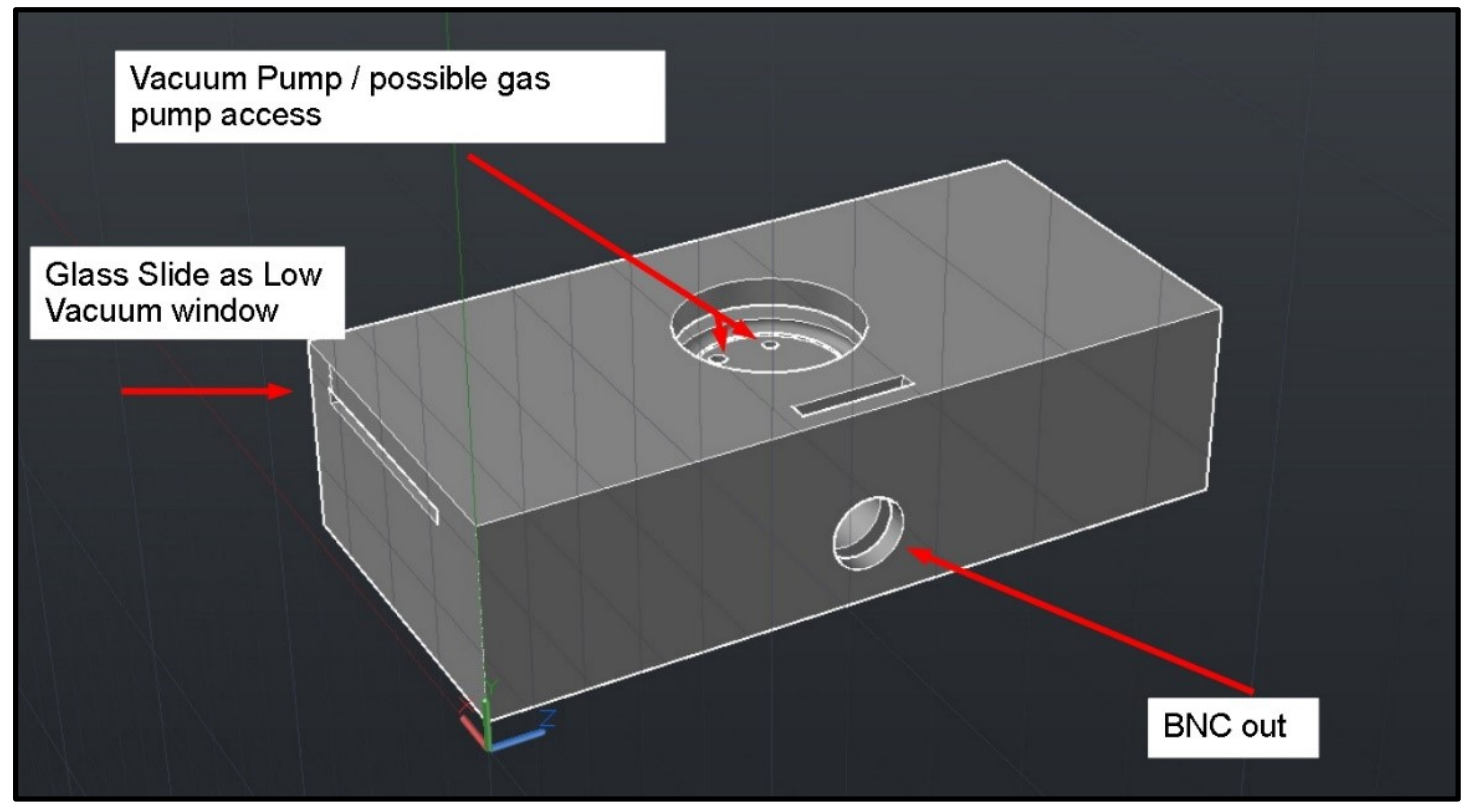

Figure 3.4: First holder design

When evaluating the requirements of the CPC technique, as well as the requirements of the stage, we developed a list of qualities the holder must have: UHV/HV capabilities, good electrical shielding, reproducible, rapid assembly, inexpensive, easy sample access, standard fittings, thin sample window $(<1 \mathrm{~mm})$. This lead to the first holder iteration, as seen in Figure 3.4. This holder used a gasket and a standard glass slide to form the low pressure/low humidity environment for the sample. The glass slide can be slid into the side, covering the sample and creating a minor vacuum environment when negative pressure is applied. There is also a slot for the BNC to screw into a nut, providing a standard fitting for the electrical signal out. This holder was made from plastic, with metal plating on the sample mount area. This design did not allow for larger sample, the plastic base did not provide enough shielding, causing highly noise data. It also was prohibitively expensive for one-at-a-time manufacturing as well as an excessive lead time. 


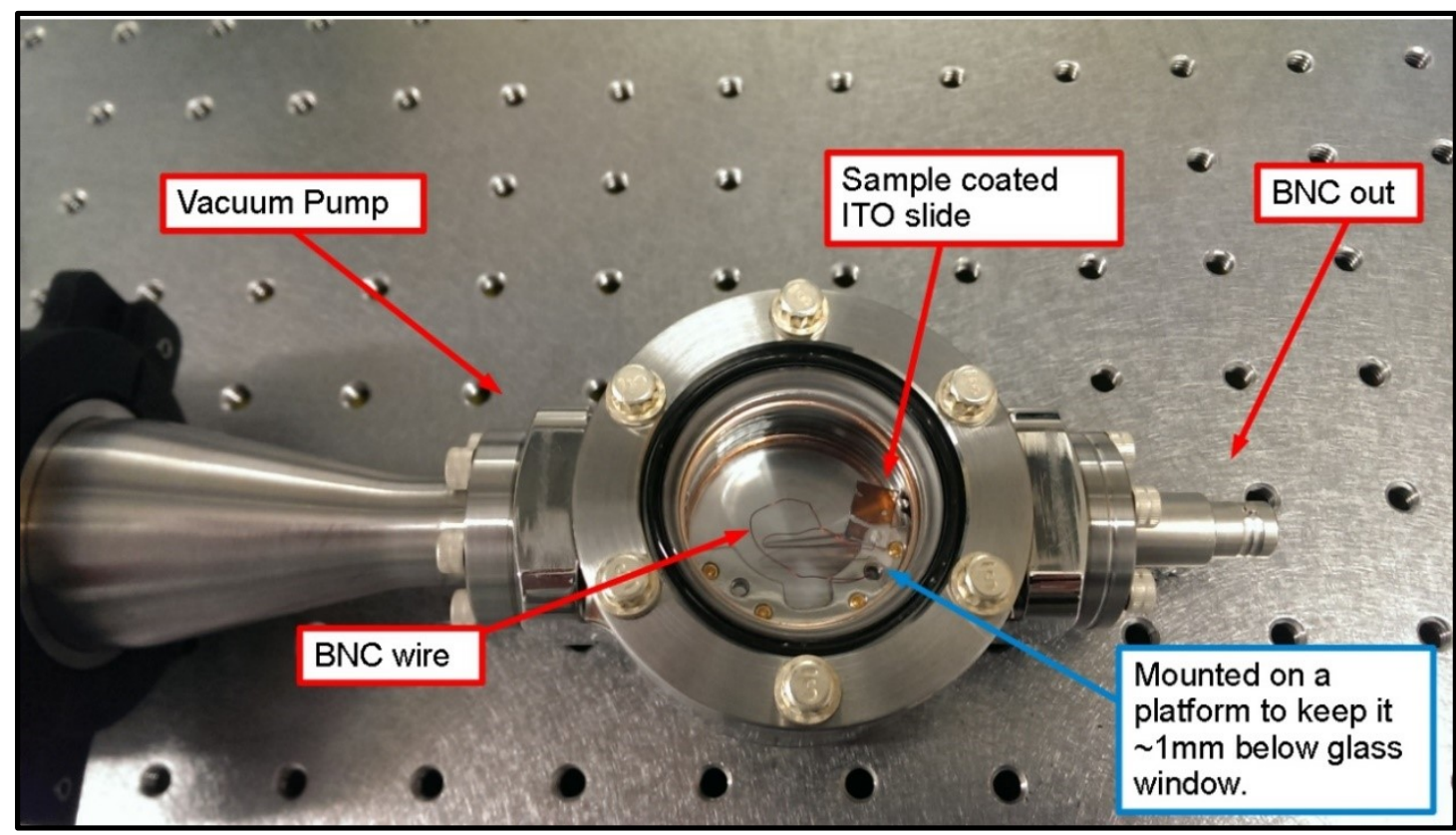

Figure 3.5 Off-the-self holder design

The second holder was designed to use only off the shelf solutions, bypassing manufacturing expenses and lead times. This design, as seen in Figure 3.5, uses standard UHV components: UHV reducer, UHV chamber, UHV window, and UHV BNC out. This design allowed for a true UHV environment for the sample. The total weight of this design did not allow the stage to function at its higher resolutions, undermining the final goal of doing sub-diffraction CPC-STED images. With a thick window and a deeper chamber, the system would only function with the system configured with long working distance objectives, once again hindering the final goal of sub-diffraction imaging. 


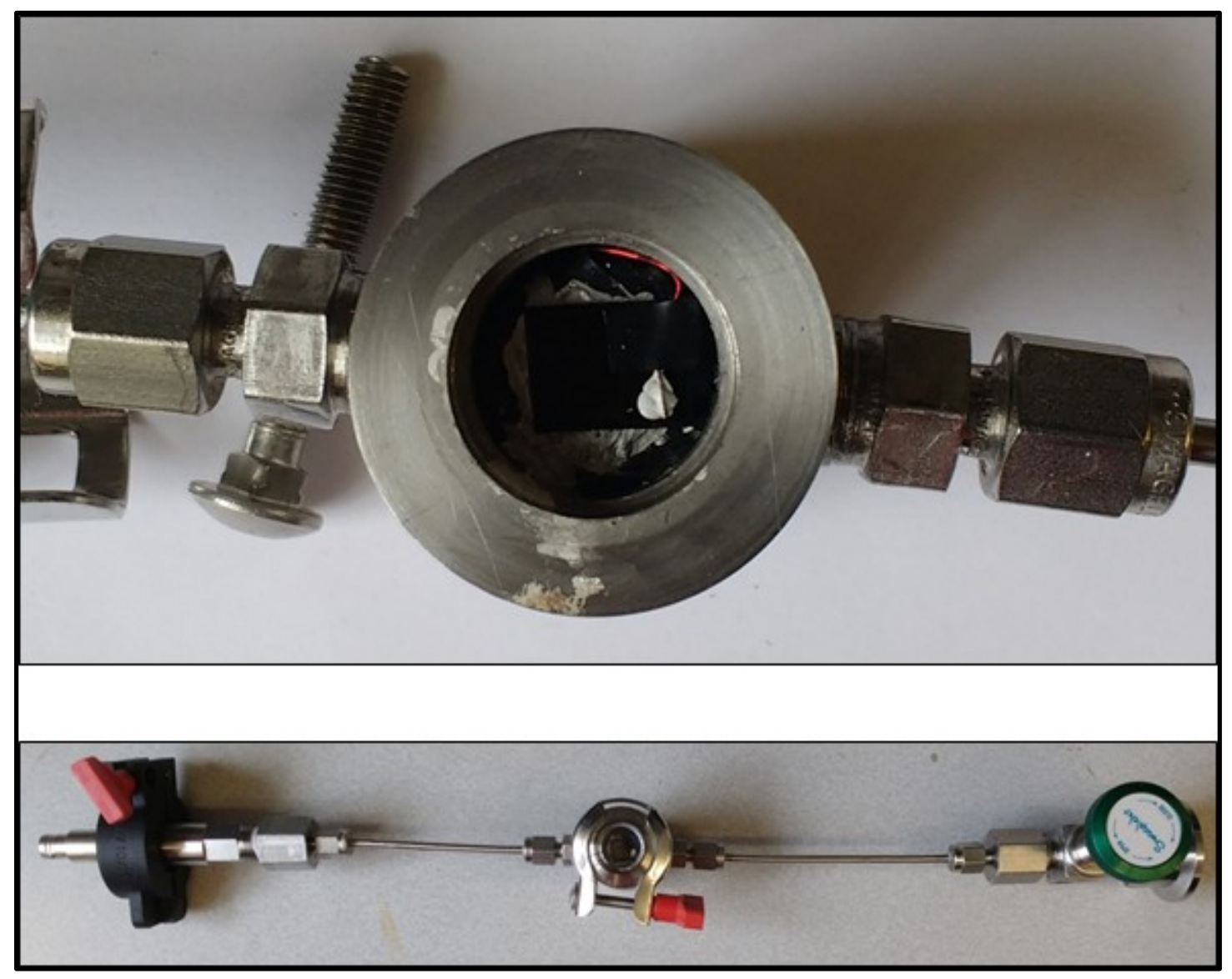

Figure 3.6: Third holder design

The third design was a balance of off the shelf and custom components. As seen

in Figure 3.6, standard UHV components are used for applying the vacuum and providing BNC out. A custom sample compartment was milled by the University of Louisville School of Ophthalmology machine shop. This holder had about a two-week lead time for the custom holder with a negligible cost. We also found an $\lambda / 4$ flat, thin window. This window allowed the use of better objectives, although the center of the window as polished flat the edges were curved slightly, causing difficulty scanning larger samples. With the long 'tails' of the holder hanging off the edges of the stage, some mechanical oscillations caused by the stage direction changes were transferred to the scans disrupting the scan quality. 


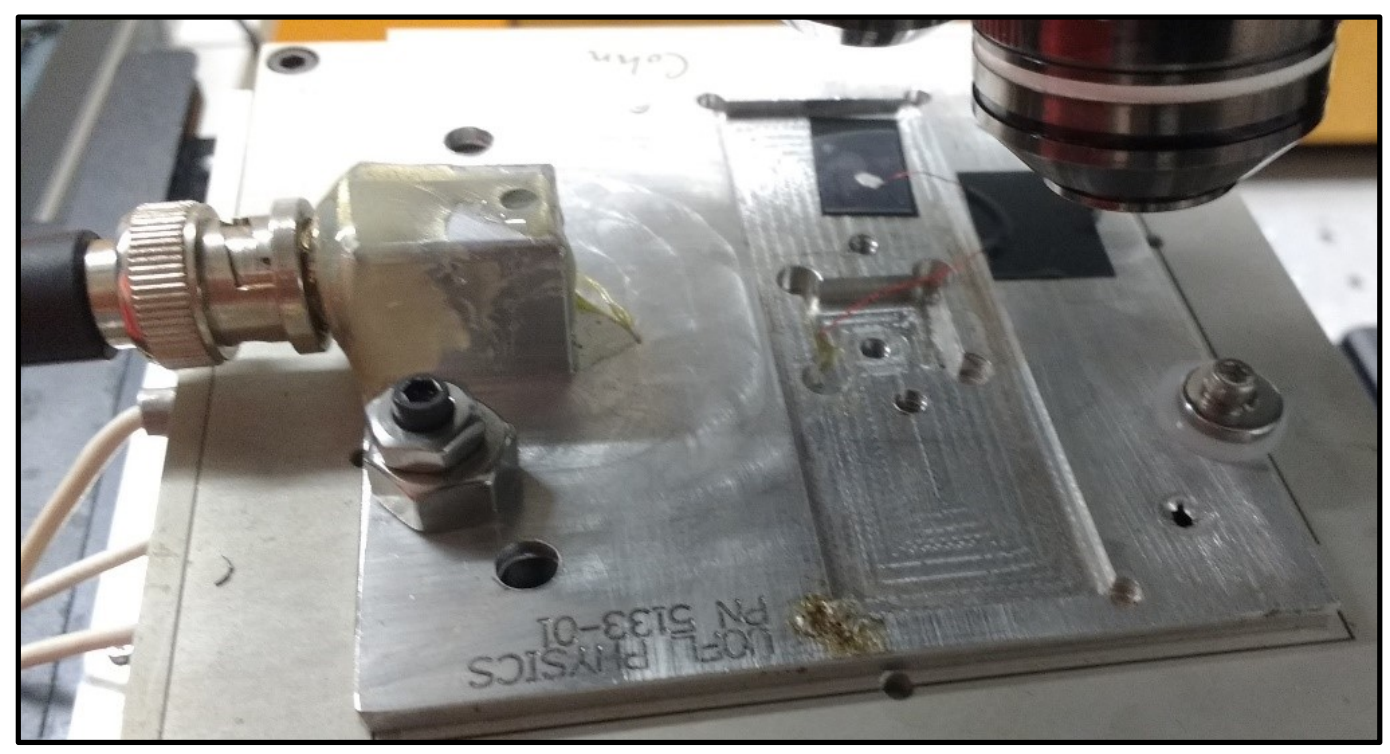

Figure 3.7: Final holder design

The final design used a completely custom design. This design was milled by Joshua Rimmer at the University of Louisville Physics Department Machine Shop. The new holder is milled from an aluminum block. It provided a connection for BNC out. While also providing room to seal a standard $1 \mathrm{~mm}$ glass slide over the sample. The glass slide can be sealed over then sample within a glove box or other similar environment. While it is not UHV, we did find it provided a low enough humidity environment for signal detection and collection. This design also mounts precisely to the stage, eliminating drift and oscillation issues. Its low profile also enables the use of the best, non-oil immersion objective we had. We eventually found that for most samples a UHV environment was not needed, thereby eliminating the need for sealing a glass slide over the sample. This development further simplified the sample preparation as we all further improved theoretical image quality by removing the air-glass-air interface. The design seen in Figure 3.7 has been iterated and adjusted for different sample requirements and minor modifications. Its onsite creation and low cost allow for this to be done easily and rapidly. 


\subsection{LabView design}

Originally, we used a piezoelectric stage with angstrom resolution [Nano-PDQ350, MadCityLabs, Inc.]. This stage had only a 45x45 um scan area. This stage also suffered from some mechanical drift issues as well as a difficulty in controlling subsequent scan areas. The MadCityLabs' stage had software and data collection software written by Dr. Thomas J. Roussel.

Currently, we are using a mechanical stage with $\sim 40 \mathrm{~nm}$ resolution $[\mathrm{H} 101 \mathrm{~A} / \mathrm{B}$, Prior Scientific]. The new stage required the development of new, custom LabView to accommodate the different communication standards used by the Prior stage. While it has a lower resolution, we do not need it since the lowest image resolution we can get with the objective and wavelength combination we are using is $\sim 70 \mathrm{~nm}$. Also, pairing the Prior Scientific stage and its proprietary software with the camera we have the scan area selection is much more accurate and intentional. With respect to the CPC-STED scans the efficiency we have improved by approximately $90 \%$ in the time needed to collect the desired data.

We created a complete, custom LabView program to hand control of the scanning stage, data collection, and image generation. This section will discuss the detail, logic, and rational for the design choices found within the program. This development is done in LabView 2009, with a Prior H101A/B stage and controller, and a National Instruments BNC 2110 breakout box the front panel contains everything that is user-controllable. The fields with red lettering above them are user editable. The scan speed in microns per second is changeable as needed, a faster scan speed is accessible for samples with faster response times, a lock-in with a smaller time constant, or other factors. Number of steps 
and scan size, in microns, set the step size. The minimum step size of the Prior H101A/B stage is $40 \mathrm{~nm}$, which is the limiting factor of the system's resolution. The loop timer, in milliseconds, sets the frequency with which the program asses the state of the system and the appropriate action to take at that time.

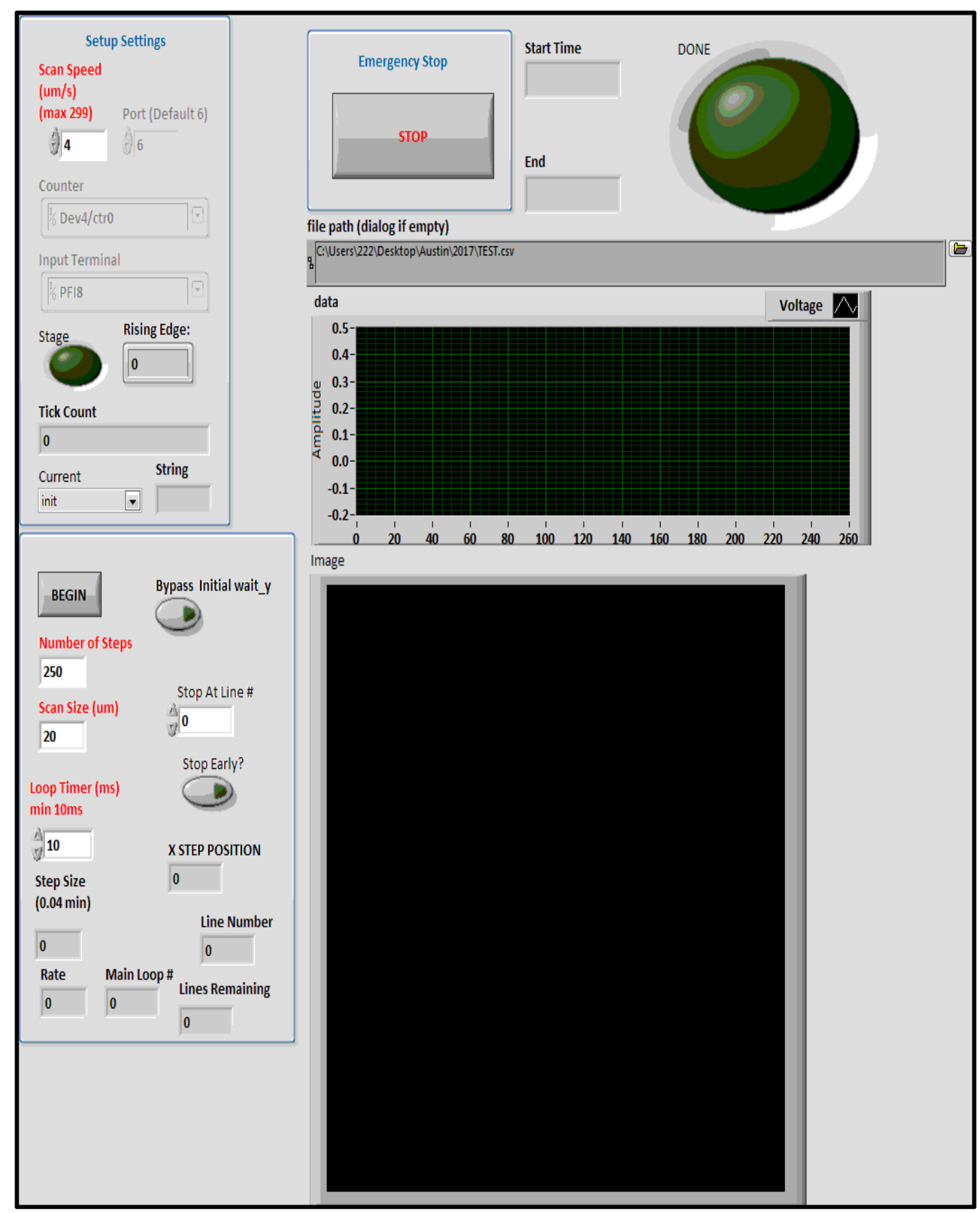

Figure 3.8: LabView front panel 


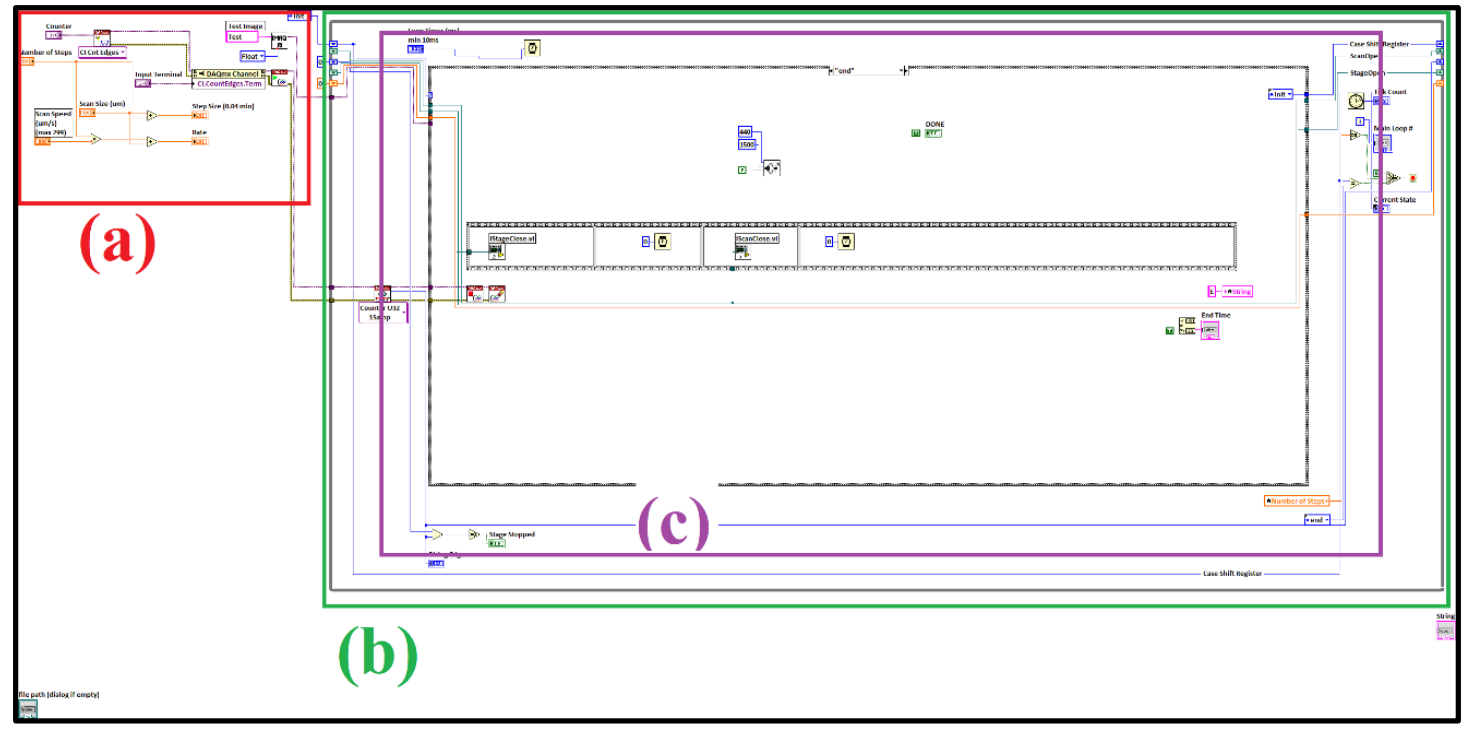

Figure 3.9: Backbone of LabView

In Figure 3.9, we have a high-level view of the LabView program. In Figure 3.9 (a) we have the user-input variable and setting. Figure 3.9 (b) is everything within the green box and everything without executes at the rate set by the user in Loop timer. Finally, Figure 3.9 (c) is a state machine. It allows for the data collection, stage control, and image building taking place appropriate intervals. What follows is a detailed explanation of the various states and the rationale behind their design. 


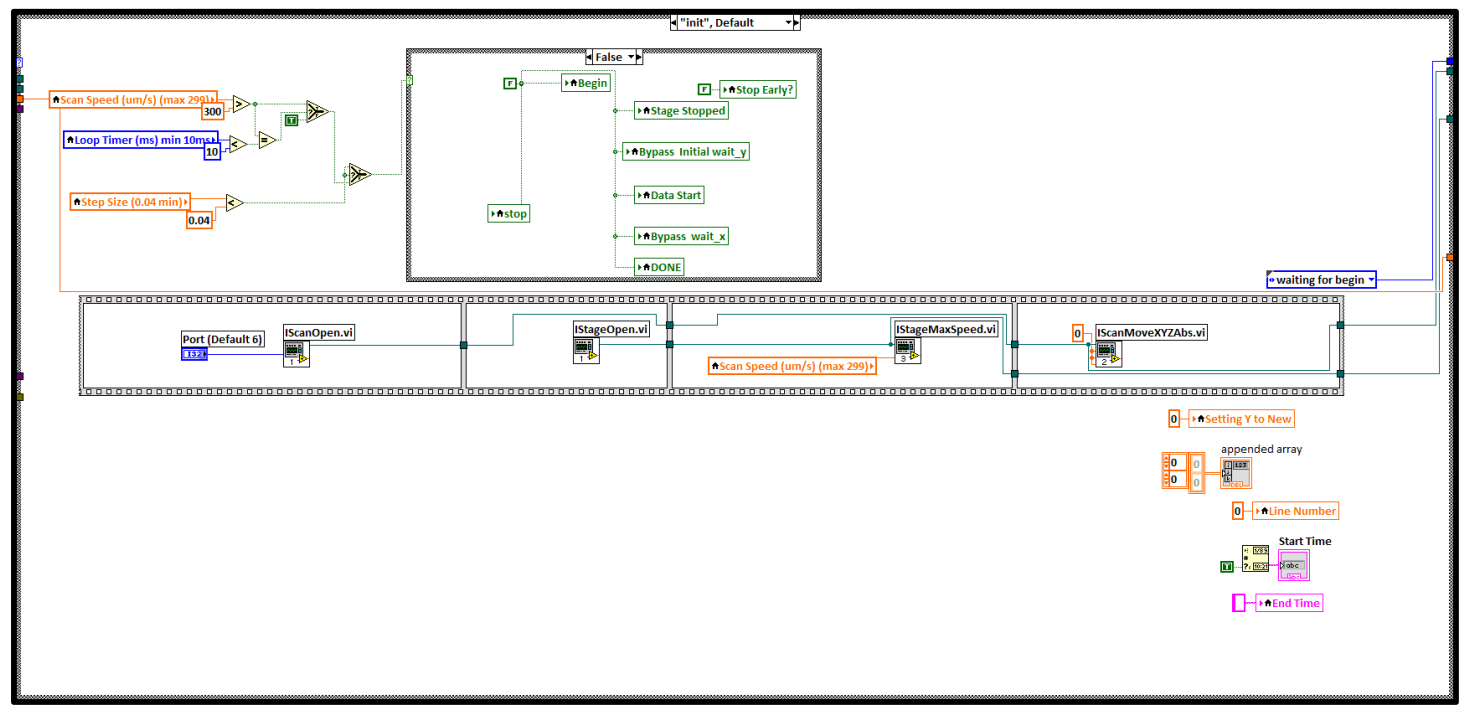

Figure 3.10: First State—“inti”, Default

The purpose of this state is to ensure the system is in a usable state. This state also imports the user settings and verifies that the setting are not values outside of the stages capabilities. Allowing invalid parameters will damage the system, this safety net/emergency shutoff system was the first to put into place to prevent system failure. This state also calls upon the call-able functions provided my Prior for this stage: IScanOpen.vi, IStageOpen.vi, IStageMaxSpeed.vi, and IScanMoveXYZAbs.vi. Where IScanOpen.vi sets the communication port and ensures the stage is ready to receive signals. Then, IStageOpen.vi prepares the stage to send position information. IStageMaxSpeed.vi sets the speed at which the stage moves during scans. Finally, IScanMoveEYZAbs.vi encodes the users scan parameters so that the stage moves in an appropriate, predictable manner. The state progresses to the "waiting for begin" state. 


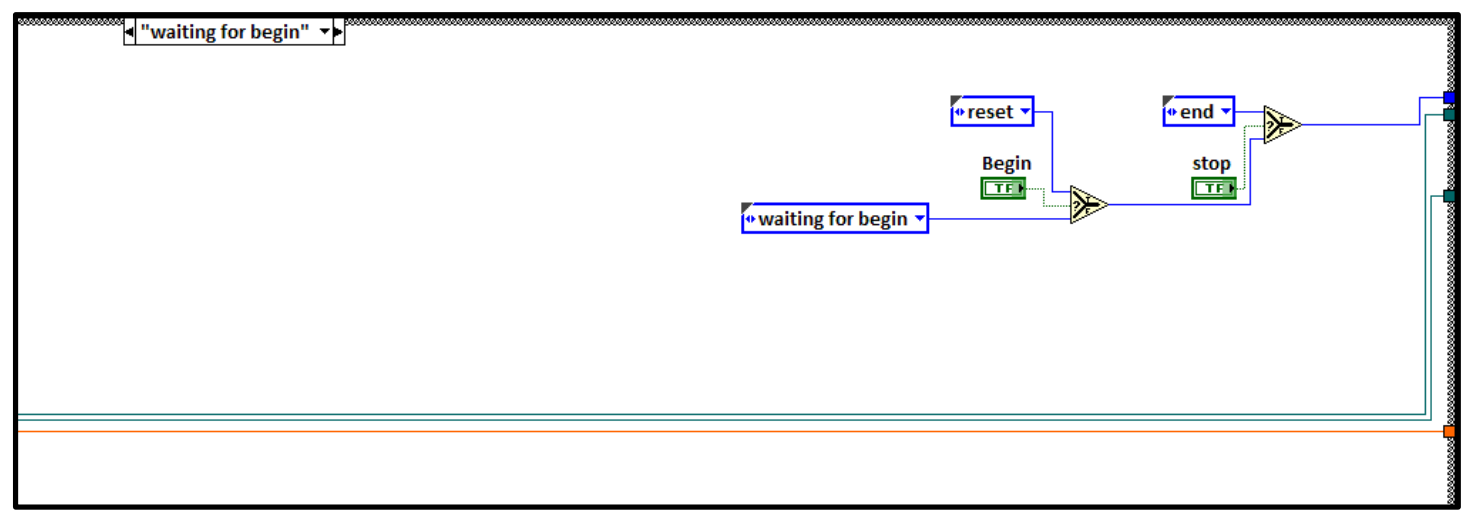

Figure 3.11: Second State-"waiting for begin"

The next state allows for the user to evaluate the system, ensuring that all parameters are appropriate. This state progress to the next state when the user presses the Begin button on the front panel.

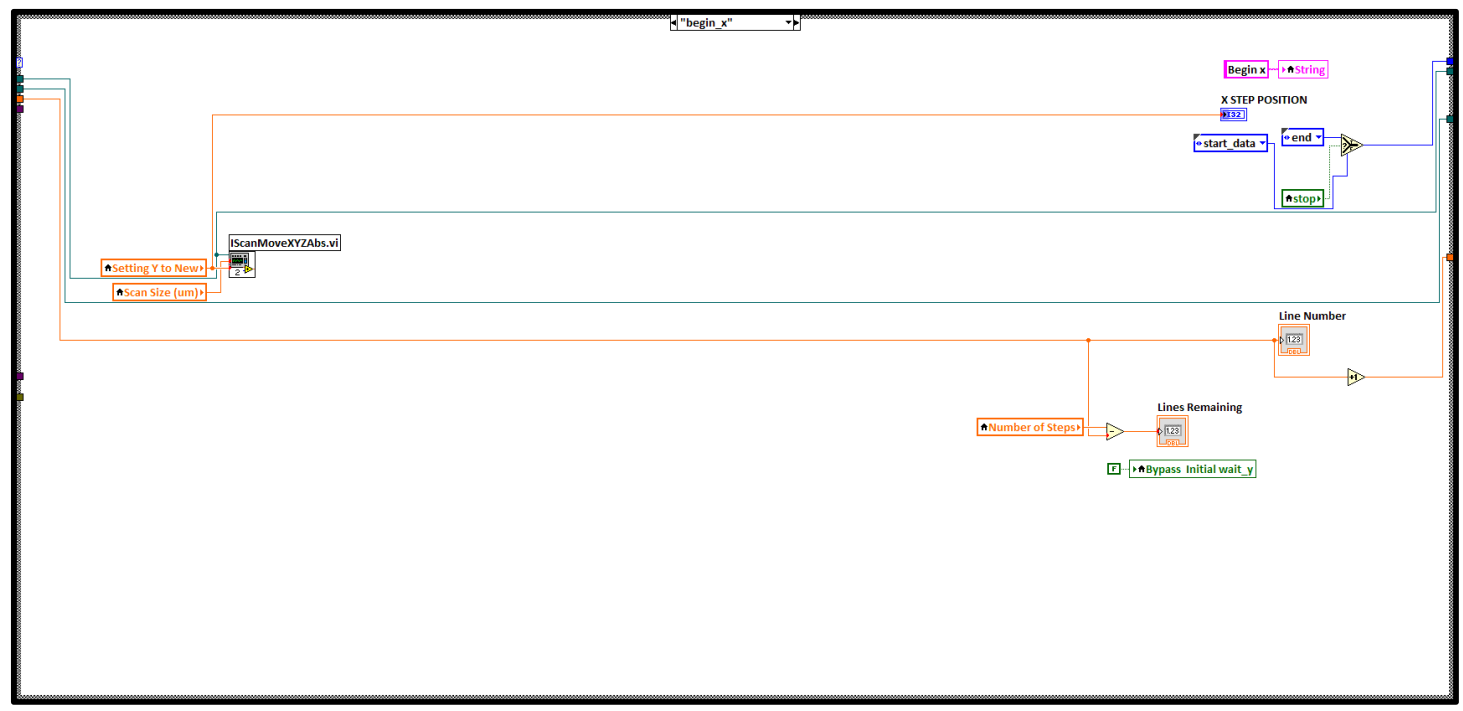

Figure 3.12: Third state_- "begin_x”"

The "begin_x" state tells the stage to begin motion in the positive $\mathrm{x}$ direction. It also sets the next state to be "start_data." 


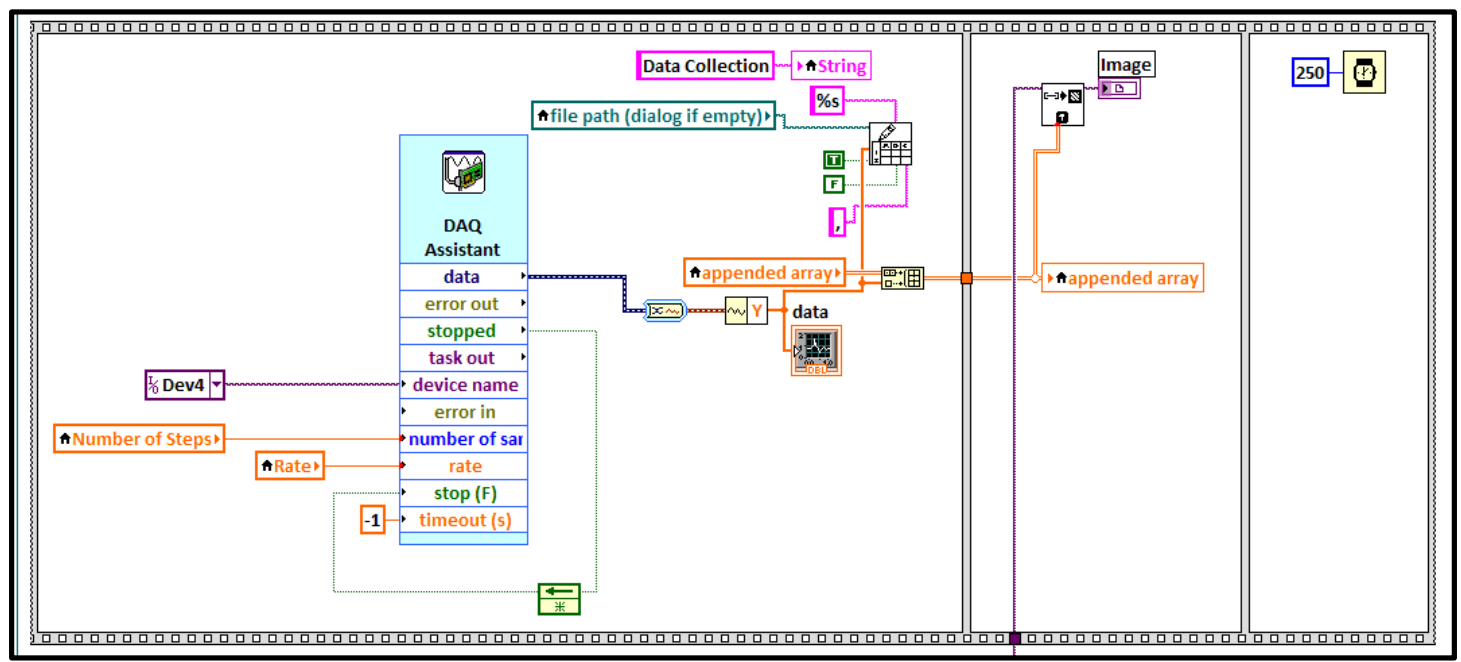

Figure 3.13: Fourth state_- "start_data”

Figure 3.13 is the core of the data collection. This data collection is a timed collection, this is because a "step-measure-step-measure" technique is poor for this stage, the mechanical drift is too noisy for that method. Yet, the stage has a good movement profile when moving at a constant velocity. Therefore, we collect data points at a rate set by

$$
\operatorname{Rate}\left(\frac{\text { points }}{s}\right)=\frac{\text { Scan speed }\left(\frac{\mu m}{S}\right) * \text { Number of steps }(\text { points) }}{S \text { can size }(\mu m)}
$$

Until we have reached the number of data points set by Number of Steps, this limiter means that the stream of data is set by the beginning the stage's motion and its initial parameters, ensuring that following scanned lines will be identical except for its $y$ positions, as is desired. The data stream is the appended to a text file in .cvs format as well as sent to LabView's internal image builder so that a user will see the image data live. Then, the system waits for $250 \mathrm{~ms}$ so that any mechanical drift of the stage will settle. Finally, system is set to wait as it returns to the $x=0$ position and the next iteration of the $y$ iteration, 


$$
y_{n}(\mu m)=n(\text { points }) * \frac{\operatorname{Scan} \text { size }(\mu m)}{\text { Number of Steps }(\text { points })}
$$

where $n$ is the next line number.
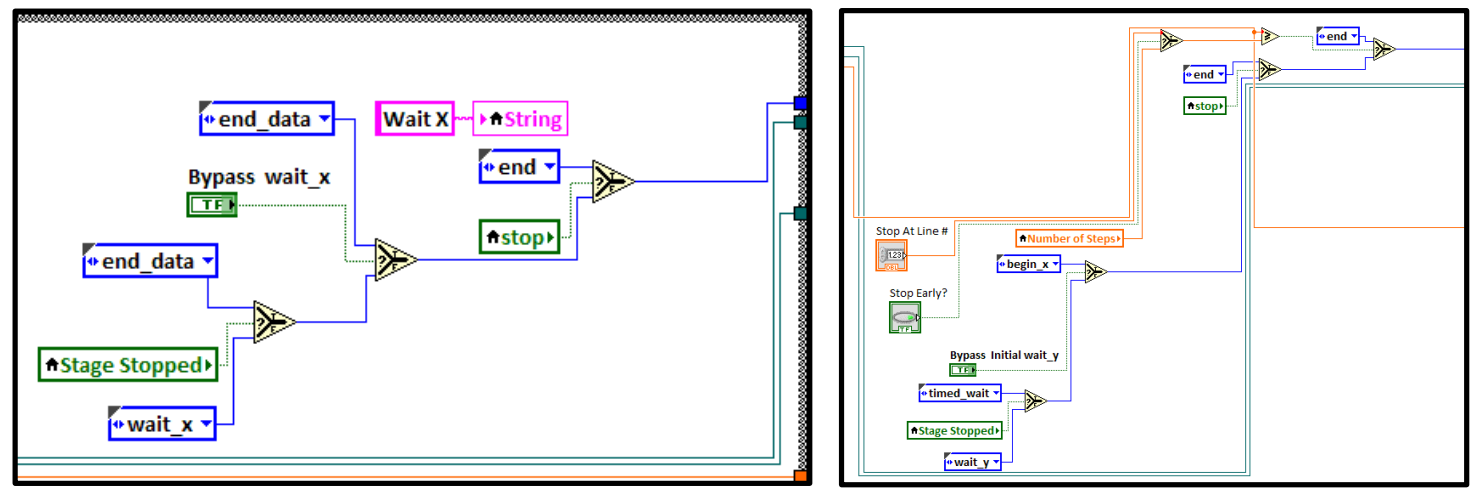

Figure 3.14: Fifth state_- "wait_x"/"wait_y"

The fifth state ensures that the state is not moving. This state also checks the Emergency Stop button as well as the Bypass "wait_x" button on the front panel. This state also ties to the "wait $y$ " state that waits for motion in the y direction to cease.

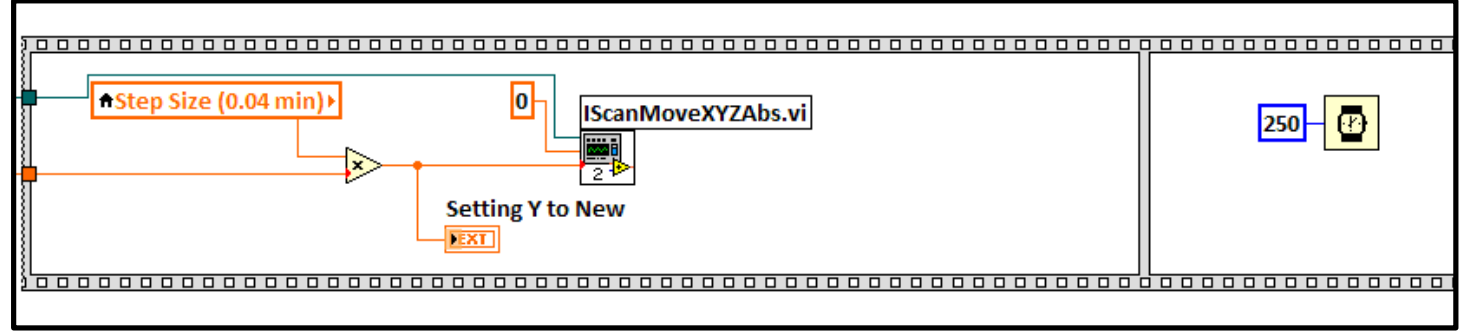

Figure 3.15: Sixth state-“reset_y”

The sixth moves the stage to $x=0$ and $y_{n}$. The system then waits again for the stage to finish moving to this new position. 


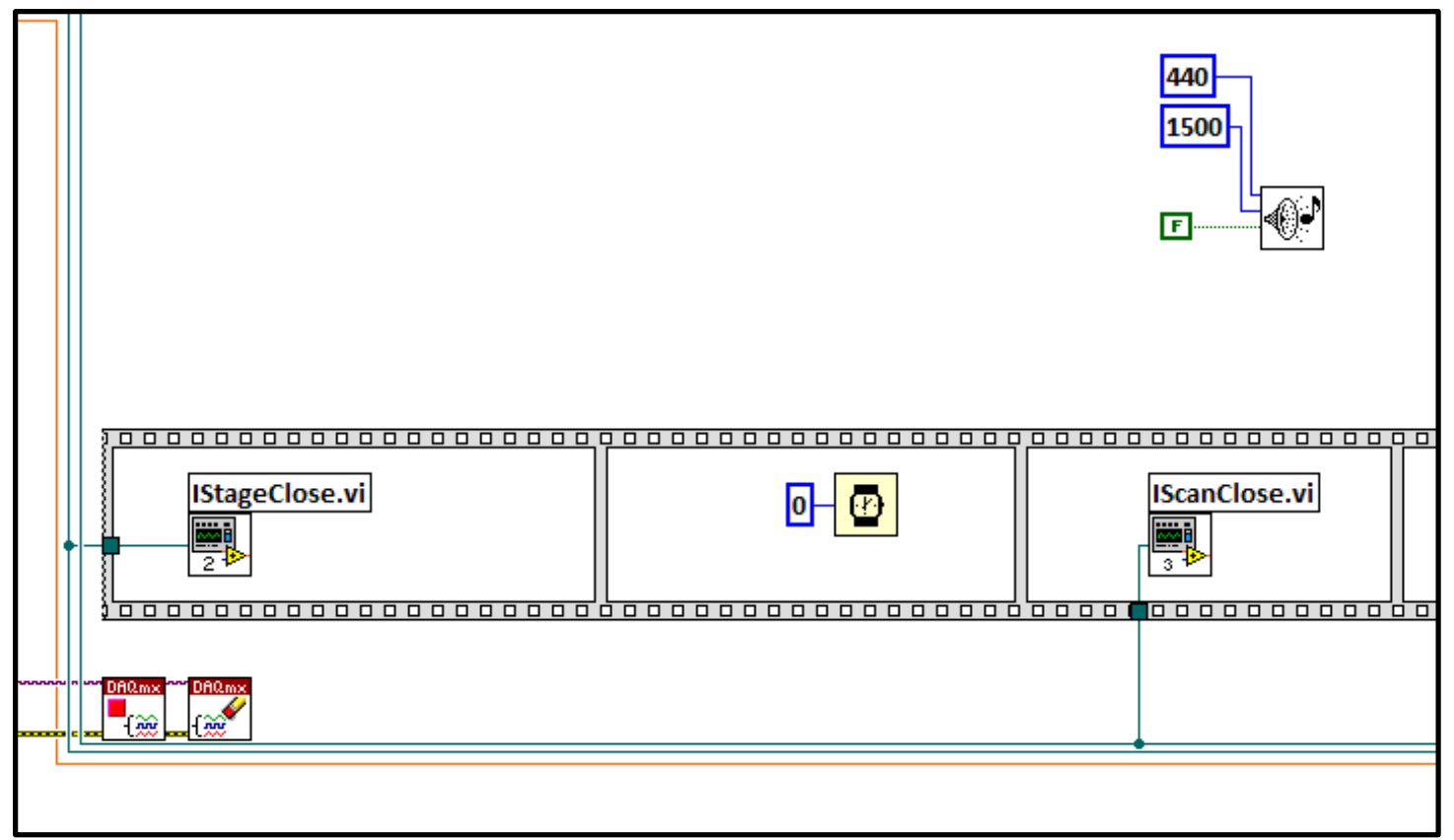

Figure 3.16: Seventh state- "end"

This state activates after the system scans its last line. It calls the appropriate, proprietary VIs to safely stop the stage from listening for new commands. It also closes the .cvs file with all the appended lines of data to prevent corruption or saving errors. Finally, it emits a tone from the computer's speakers informing users of the system's state without constant, visual monitoring. 


\section{CHAPTER 4 RESULTS OF THE SCANNING-CPC SYSTEM}

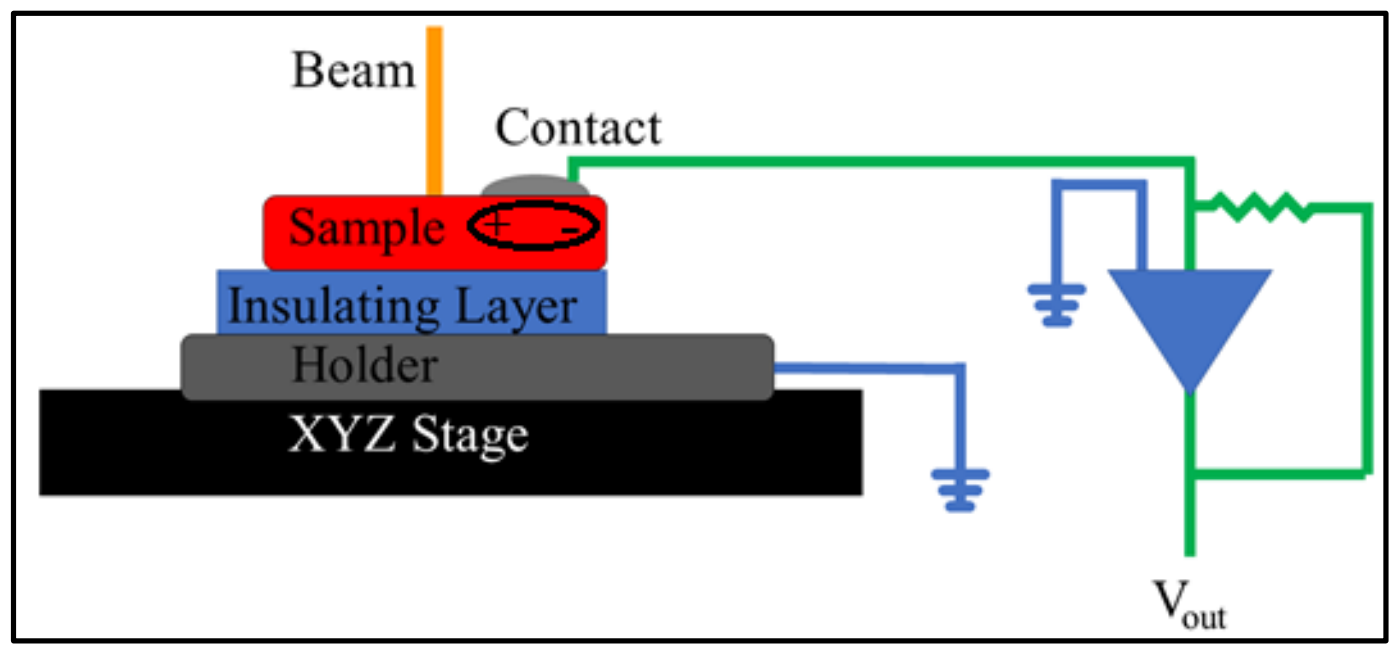

Figure 4.1: Scanning-CPC layout and design.

Capacitive-photocurrent (CPC) measures the AC electric field of a sample when a

pulsed or chopped light source is focused on the sample, generating electron-hole pairs which can then separate to create a measurable dipole field. The CPC technique detects a combination of the sample's absorbed light and the amount of charge separation within the sample, with a reasonable range in the picoamp regime seen in the calculations done in Chapter 2.3. The higher a sample's absorbance, the more electron-hole pairs are available to separate. This understanding means that a scanning-CPC technique could be developed which could provide information on a sample's defect density since an increase in defects should inhibit absorbance, how its geometries affect electron-hole pair generation and separation, a basic measure of material quality, or two-dimensional information on what and how the electric fields are made and rectified. 


\subsection{Preliminary measurements}

Since most of our previous studies were done using the OPA system with a $1 \mathrm{kHz}$ repetition rate, we needed to know how the frequency of the probing beam effects the signal we measure since we will be replacing a pulsed light source to a mechanically chopped CW light source. Using the standard scanning-CPC setup seen in Figure 4.1, we applied the $2.54 \mathrm{eV}$ beam, adjusting the chopper frequency. We see from Figure 4.2 that there is a significant dependence of the CPC signal on the probe beam's chopped frequency. We also adjusted the beam's power, maintaining a constant chopper frequency at $1 \mathrm{kHz}$. In Figure 4.3, we see that the CPC signal has a linear dependence on the power of the excitation beam. This is as expected, leading us to maximize image quality by

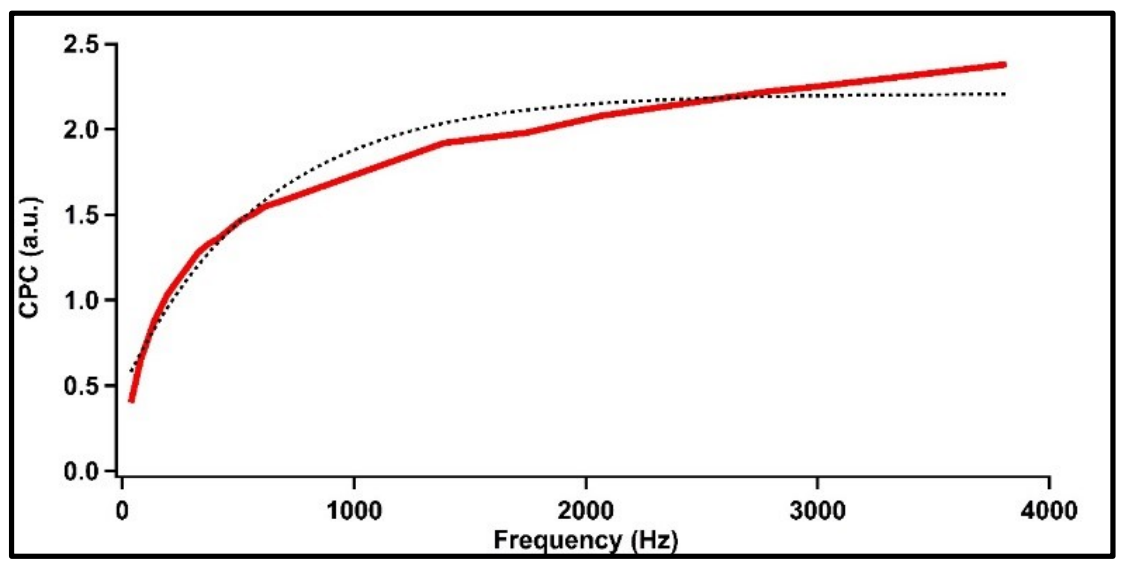

Figure 4.2: CPC signal as a function of chopper frequency.

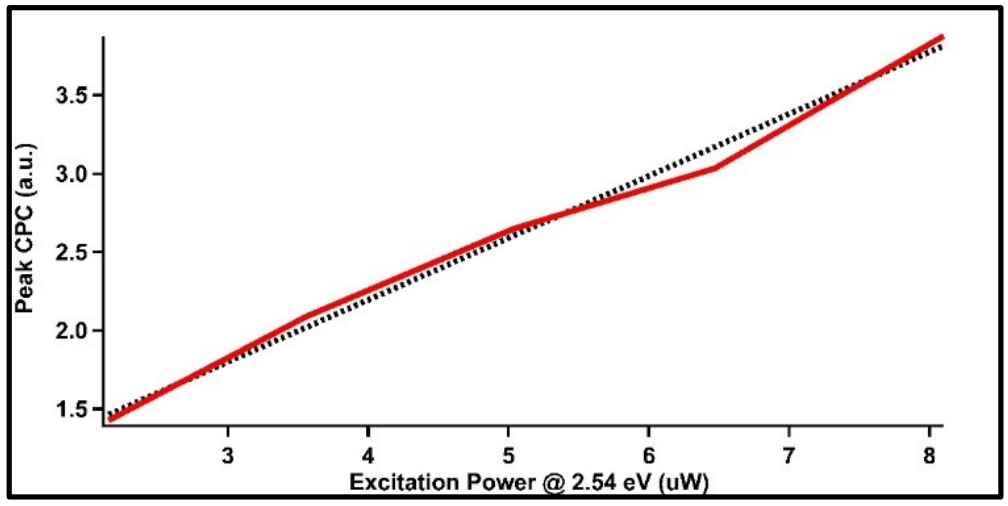

Figure 4.3:CPC dependence on 2.54eV excitation beam power. 
using higher frequencies on the chopper and high powers for the excitation beam since the higher frequencies increase the signal fidelity through the lock-in.

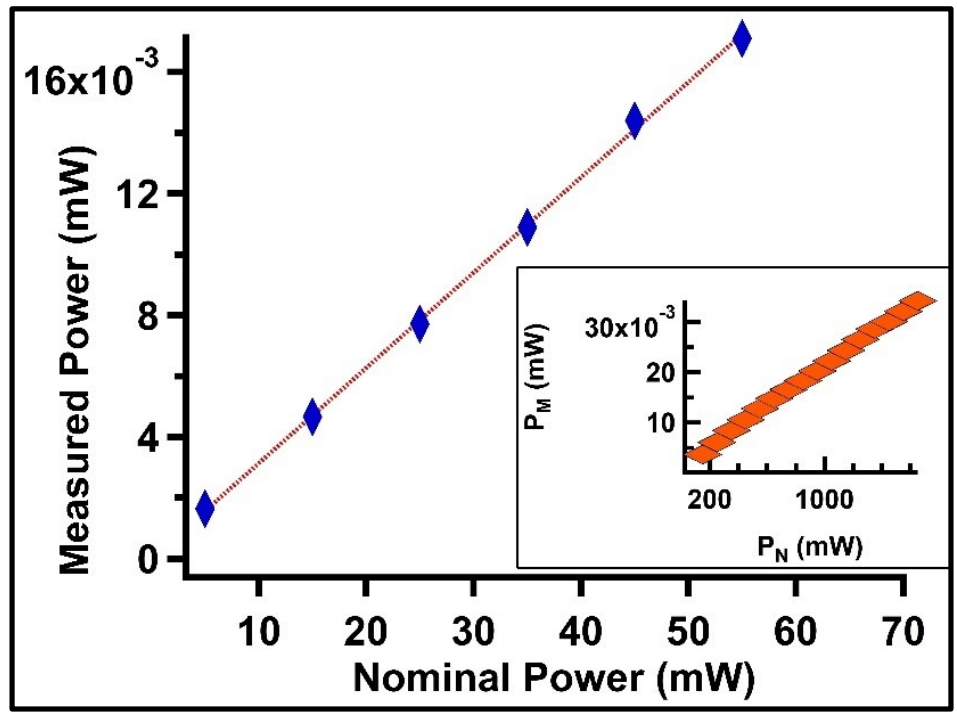

Figure 4.4: Comparisons of nominal (software reported) laser power versus the sample surface measured power for the $2.54 \mathrm{eV}$ beam and $2.09 \mathrm{eV}$ beam (inset).

Some of the most crucial components of a scanning system are the light source and the power output of the light source. Figure 4.3 shows the power of the beam, as measured by a Newport 1916-R power meter. These are the powers measured after the microscope's objective, the power that is incident on the sample's surface, rather than the output power reported by the laser's software. The real power is expected to be significantly less than the nominal, or software reported power, since there are many vectors for power low throughout the system. In Figure 4.4, we have the plots comparing the nominal power and the power measured at the sample's surface for both the $2.54 \mathrm{eV}$ and $2.09 \mathrm{eV}$ beams. They both show a linear dependence, which is good, because as long as we are referring to the ratio of the powers, they are interchangeable in our discussion. We have a fit equation for both scenarios: $P_{M(2.54 \mathrm{eV})}(\mathrm{mW})=6 * 10^{-6}+3.13 * 10^{-4} *$ 
$P_{N(2.54 \mathrm{eV})}$ and $P_{M(2.09 \mathrm{eV})}(\mathrm{mW})=1.32 * 10^{-3}+2 * 10^{-5} * P_{N(2.09 \mathrm{eV})}$, where $P_{N}$ is the nominal power for each laser and $P_{M}$ is the measured power. With these results, we were able to begin scanning with realistic expectations for the needed power, the power available, and the frequency needed to successfully scan images.

\subsection{Preliminary scans}

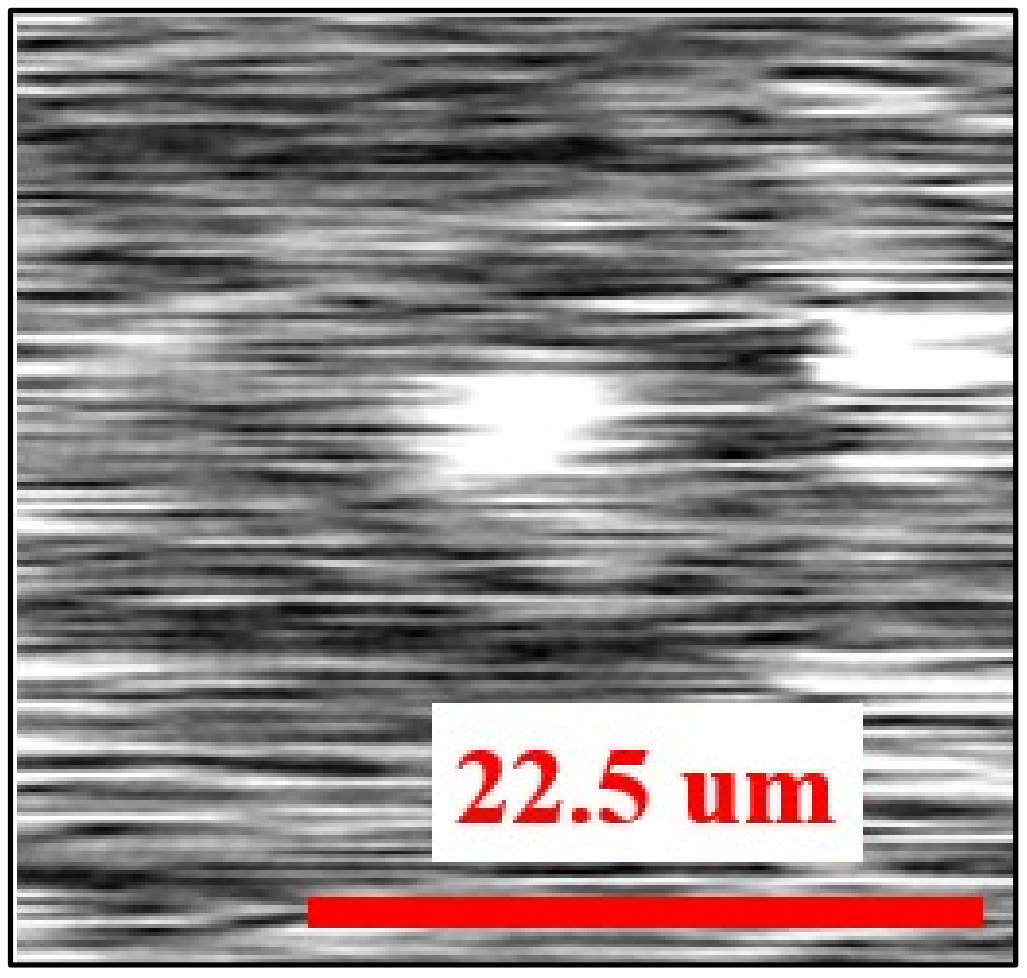

Figure 4.5: CPC image of a PCBM clump with a $2.54 \mathrm{eV}$ light source.

Upon creating a satisfactory holder (the third iteration, Figure 3.6) and with software in place, we were ready to begin testing this technique. The original software was written by Dr. Thomas Roussel to collect fluorescence data from standard STED microscopy. This software was modified to accept the CPC signal in place of the STED's photomultiplier signal. This change allowed for the creation of CPC image. The first material we tested was PCBM, which was spin coated onto an ITO coated glass slide. The PCBM was spin coat at a low RPM, 100 RPM, to ensure a thick layer and maintain 
some of the larger particle clumps. This procedure was implemented because we anticipated the first scans to have lower resolutions. By using this method, a larger, planned anomaly would be easier to find and resolve into a CPC 2D image. In Figure 4.5,

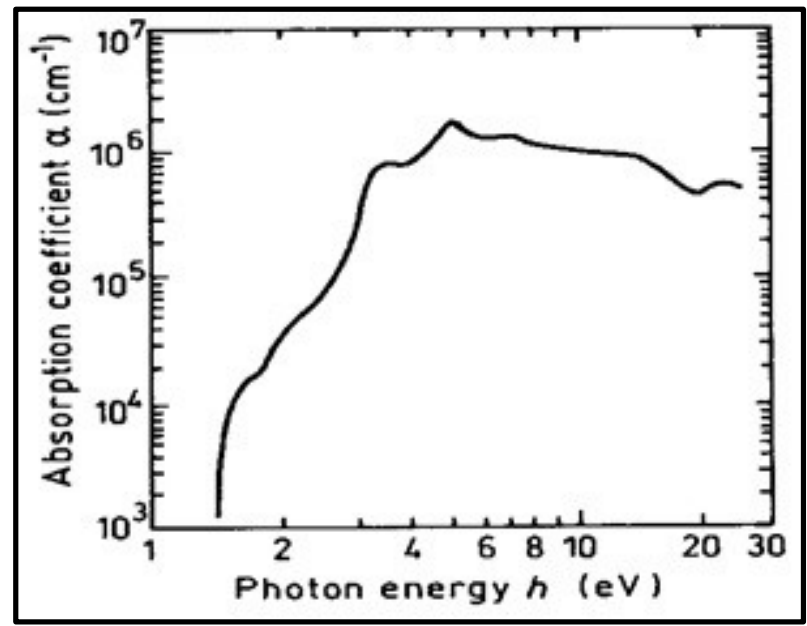

Figure 4.6: Absorption coefficient for GaAs [10]

we have the first CPC image that showed any information that was resolved above the noise. The CPC image shows a distinct high signal region in the center of the image. This scan revealed that PCBM can be resolved into a 2D image since it is a sample well known to respond to $2.09 \mathrm{eV}(592 \mathrm{~nm})$ and $2.54 \mathrm{eV}(488 \mathrm{~nm})$, as seen from our previous published work discussed in Chapter 2.3. This situation is where a thicker, poorlydispersed PCBM sample helped with early testing, providing signals in the picoamp regime.

With this promising result, we decided to test GaAs particles. We did this by suspending large ( $>15 \mathrm{um})$ diameter GaAs pieces in a solution and spin coating onto ITO coated glass slides at low RPM. Figure 4.7 shows an SEM taken of one of these GaAs crystals. Although the sample has good absorption in both $2.09 \mathrm{eV}$ and $2.54 \mathrm{eV}$ as shown in Figure 4.6, no distinguishable images could be generated. This result could be due to a few factors. The incident light would excite the top surface of the GaAs crystals which is 
$>5$ um from the ITO interface, unlike the PCBM which was significantly thinner. Also, the GaAs does not rest uniformly on the ITO surface since the GaAs has a rough surface, further limiting the ability to measure the electric field through the contact on the ITO surface.

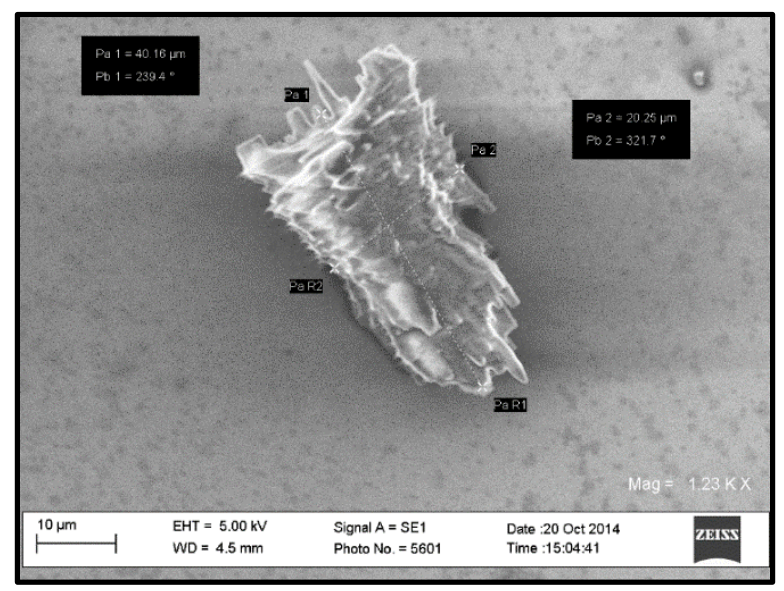

Figure 4.7: GaAs SEM image

Seeing that the PCBM provided initially promising results and the GaAs did not provide any usable images, we narrowed our focus on thin materials that could be readily dispersed on a ITO coated slide or semiconductors. The dispersed samples allow for direct material analysis, where testing with semiconductors allows for a care tuning of parameters that we are testing.

\subsection{Perovskites}

Perovskites are a class of materials derived from calcium titanium oxide. We had a group member, Kasun Fernando, study the effects of perovskite structure on efficiencies and electro-optic responses of solar cells. This availability meant that we had wellcontrolled samples and sample preparation techniques. With their fantastic optical and electrical properties, the perovskite material system is promising for applications such as solar cells, detectors, and light emitting diodes. We used a perovskite of the form $\mathrm{CH}_{3} \mathrm{NH}_{3} \mathrm{PbI}_{3}$. 


\subsubsection{Preliminary work}

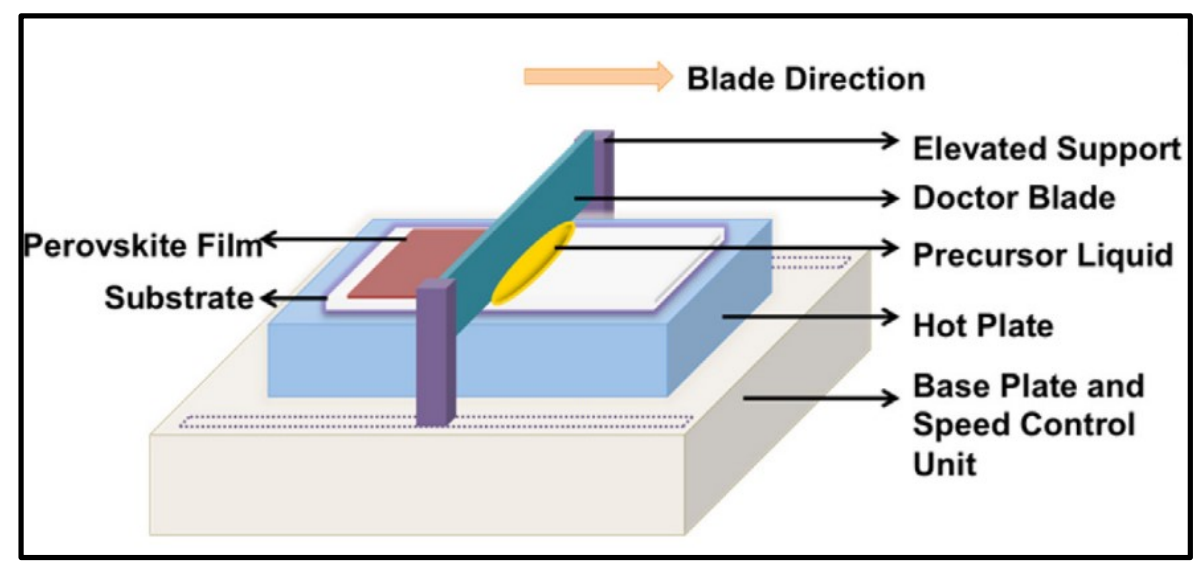

Figure 4.8: Perovskite fabrication diagram [17]

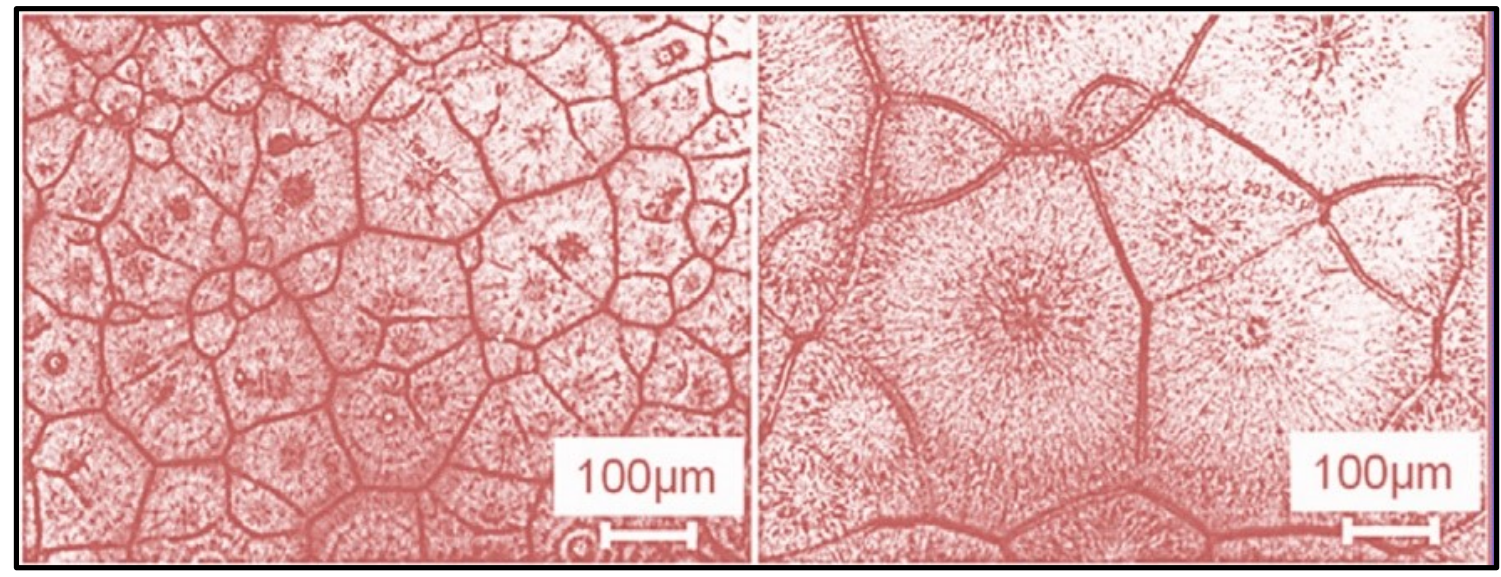

Figure 4.9: Optical image of perovskite deposited films. [17]

To create a perovskite layer for study, we used solution-processed organicinorganic hybrid perovskite cells applied through the doctor blading method [32]. The solution is formed by dissolving lead iodide $\left(\mathrm{PbI}_{2}\right)$ and methyl ammonium chloride (MACl) in dimethylformamide (DMF) at a 1:1 molar ratio. This solution is stirred at $70^{\circ} \mathrm{C}$ for at least 2 days then doctor bladed onto ITO coated glass slides. The size of the perovskites is controlled by the temperature of the substrate during the doctor blading, shown in Figure 4.8. This method provides a sample as seen in a photograph in Figure 4.9, where we have both small (left) and large (right) formations. 


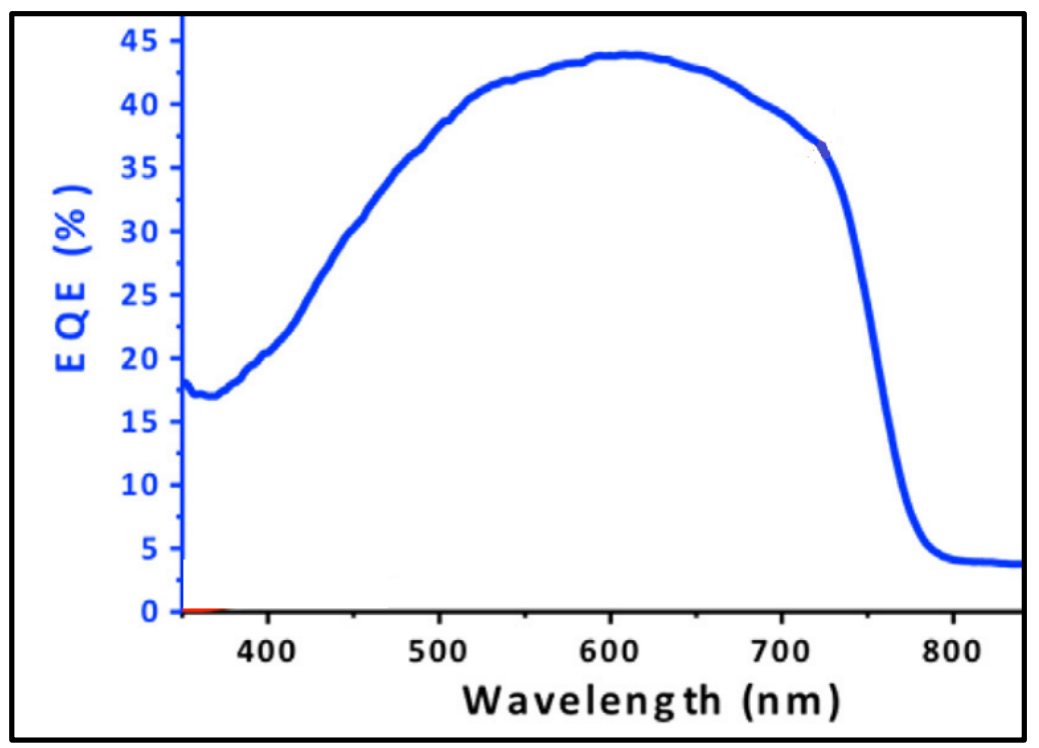

Figure 4.10: External quantum efficiency and integrated short circuit current density of perovskite. [17]

Much of the breakthrough work on perovskites was done by using the perovskite as a visible sensitizer in a dye-sensitized solar cell. Perovskites as a thin film have provided solar cell efficiencies greater than 20\% [17]. With their low cost and new discovered uses, we decided to investigate this material system with the scanning-CPC technique. We see in Figure 4.10 that this perovskite system has high EQE at both $2.54 \mathrm{eV}(488 \mathrm{~nm})$ and $2.09 \mathrm{eV}(592 \mathrm{~nm})$, the primary wavelengths in the STED setup. We also see from Figure 4.11 that the absorption coefficient, shown on the black trace, indicates that at

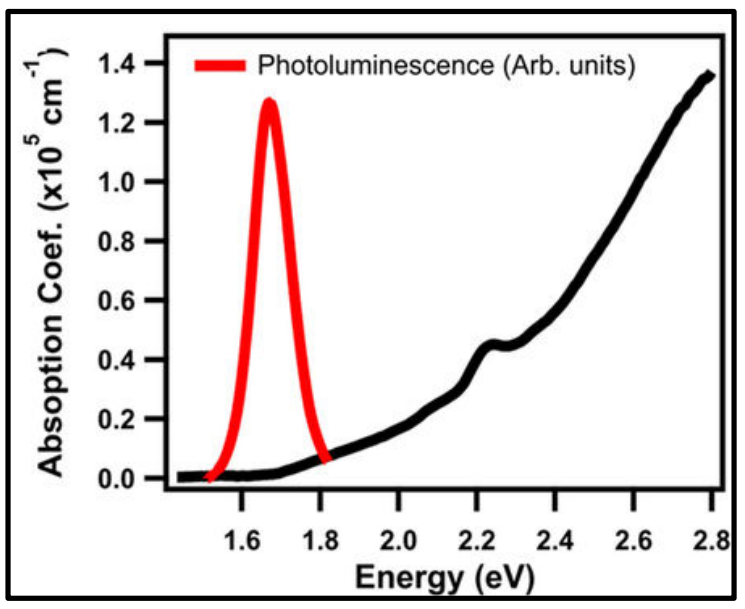

Figure 4.11: PL and absorption coefficient for perovskites [22] 
both $2.09 \mathrm{eV}$ and $2.54 \mathrm{eV}$ should provide sufficient CPC signal for either of these energies. This result lead us to do a CPC spectrum of this perovskite system, shown in Figure 4.12, this spectrum was generated using the setup previously described in Ch. 2 . The red trace shows the $\mathrm{CPC}$ response, with strong responses at $2.09 \mathrm{eV}$ and suggests there would also be a good CPC signal at $2.54 \mathrm{eV}$. With these details, we proceeded to use the CPC-STED system to generate images of the perovskites.

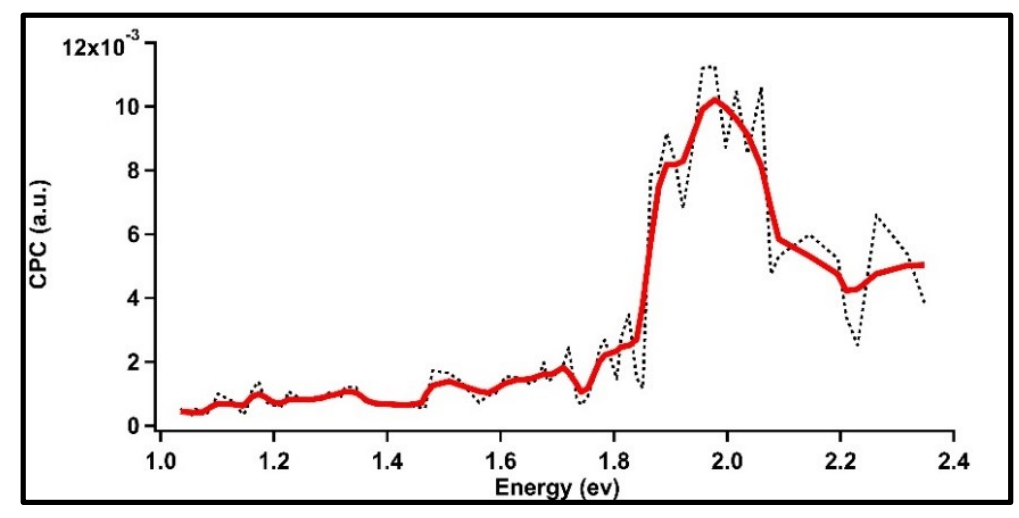

Figure 4.12: CPC signal of perovskite system. 


\subsubsection{Perovskite scans}

By using the $2.09 \mathrm{eV}$ beam without the phase plate, the chopped gaussian beam produces the 2D CPC image shown in Figure $4.13(\mathrm{~b})$. We can see how this matches a photograph taken through the confocal microscope using a white light source in Figure 4.13 (a). There are a few things to note: from the photo, the brighter regions in (a) imply low absorption, which should indicate a region that would have a low CPC signal.

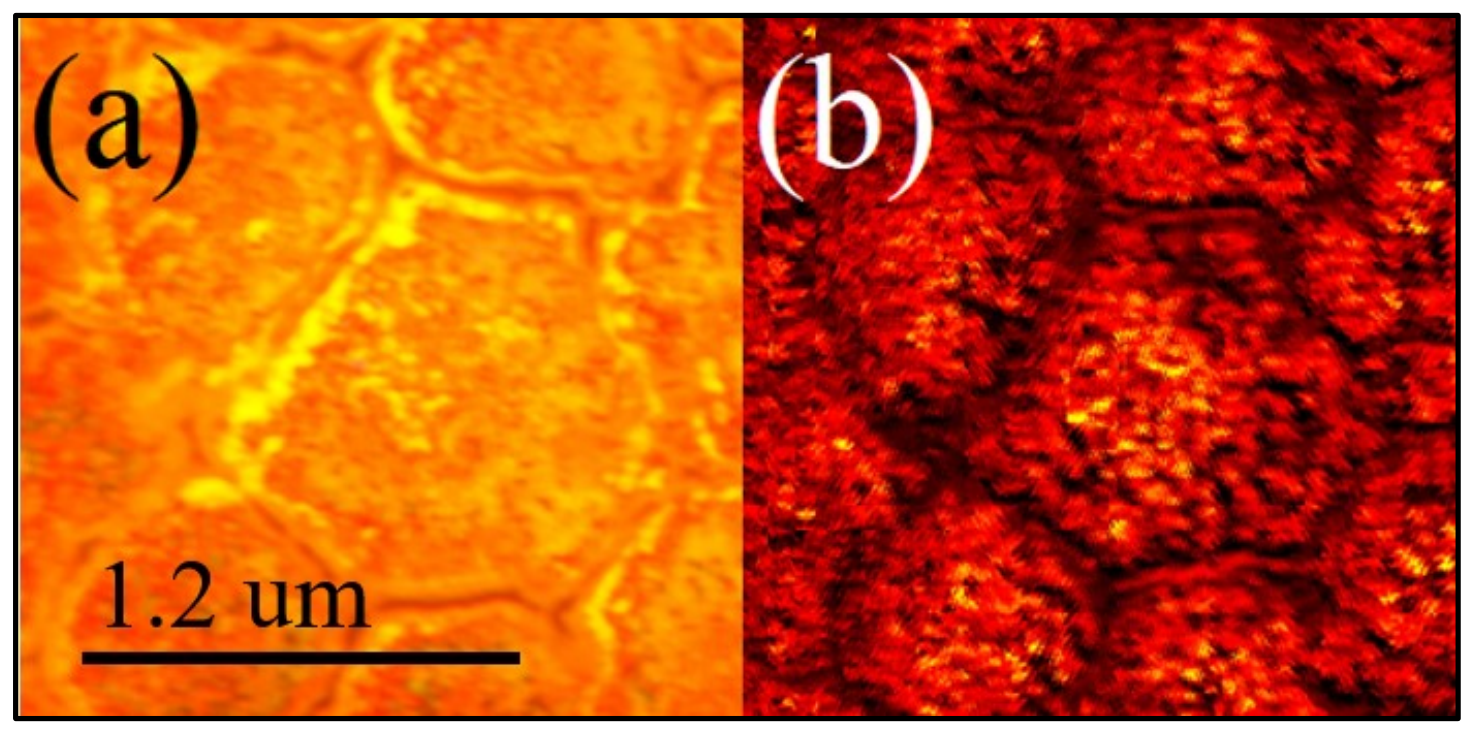

Figure 4.13: (a) photograph and (b) CPC image of perovskites

Likewise, a dark region in (a) implies higher absorption which should be a high CPC signal region. Yet comparing (a) with (b), we see regions that follow and oppose these assumptions. This early work shows that $\mathrm{CPC}$ has potential for providing novel information. Within the scans of the small perovskite formations, we see a fairly uniform CPC signal with a sharp drop-off on the domain walls. We then compared the small perovskites to large perovskite formations, as seen in Figure 4.15. Comparing the smaller perovskites in Figure 4.13 with the larger perovskites in Figure 4.15, we see a significantly higher CPC signal in the center region of each of the formations. In the 2D CPC image, we see a correlation between the darker regions of the center of Figure 4.15 


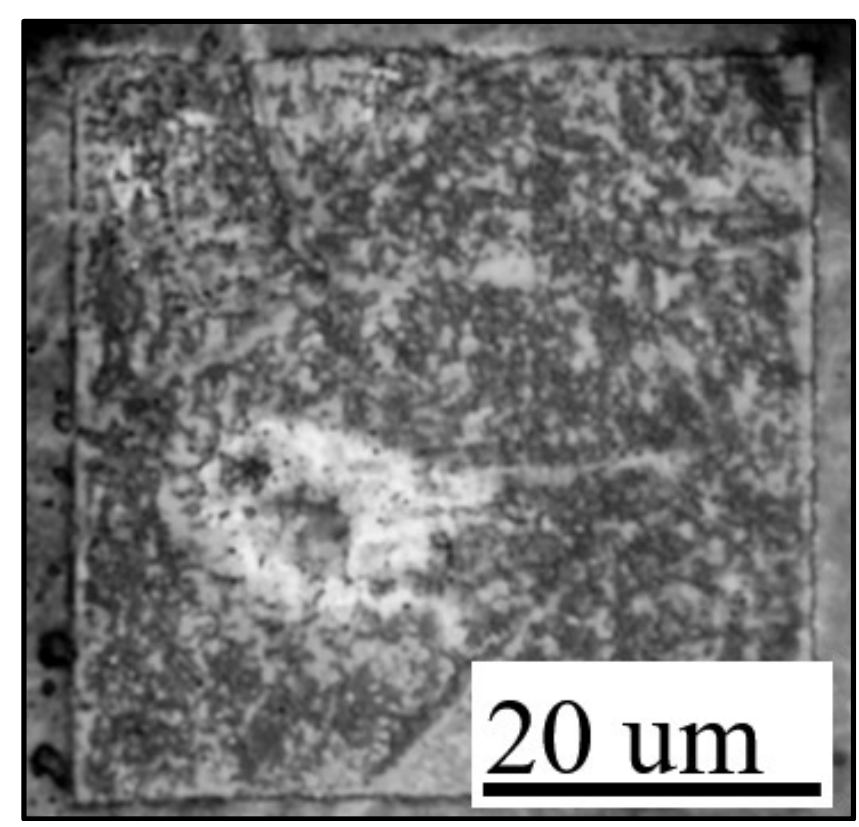

Figure 4.14: Burnt large perovskite formation after CPC scan.

(a) with the brightest region of Figure 4.15 (b). Also, the CPC shows low signal regions that do not correlate to anything seen in the photo, specifically the horizontal striations that radiate out from the center of the perovskite formation in Figure 4.15 (b).

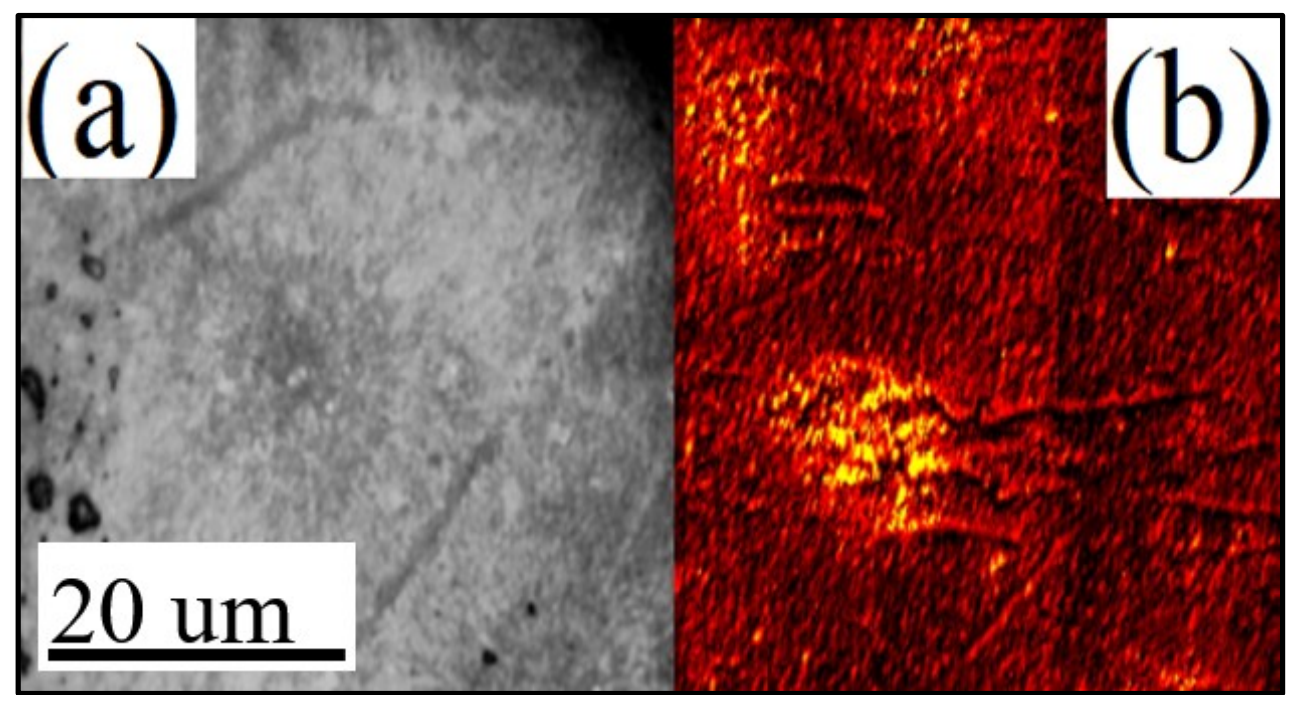

Figure 4.15: (a) Photo versus (b) CPC of large perovskite formation

These scans were done in open air to reduce any interference from a glass slide.

This configuration means that sample degradation was a consideration both from being in open air and from damage caused by the laser [33]. Figure 4.14 revealed that the CPC scans were damaging the samples, which is the same area as Figure 4.15. Figure 4.14 also 
reveals the horizontal striation that was seen in the CPC image. This burning from the scan also reveals the center of the larger perovskite formation center. This result lead us to consider samples that are not as sensitive to environmental factors.

\subsection{Gallium Nitride}

Gallium nitride $(\mathrm{GaN})$ is a semiconductor with a Wurtzite crystal structure and a direct band gap at $3.4 \mathrm{eV}$. Since we were seeking stable samples, we settled on GaN because it is well-known with a significant body of work describing its properties. The collaboration with another member of our group, Sowmya Kolli, who also studies GaN, provided me with samples and her with potentially material property data derived from the CPC results. 


\subsubsection{Preliminary work}

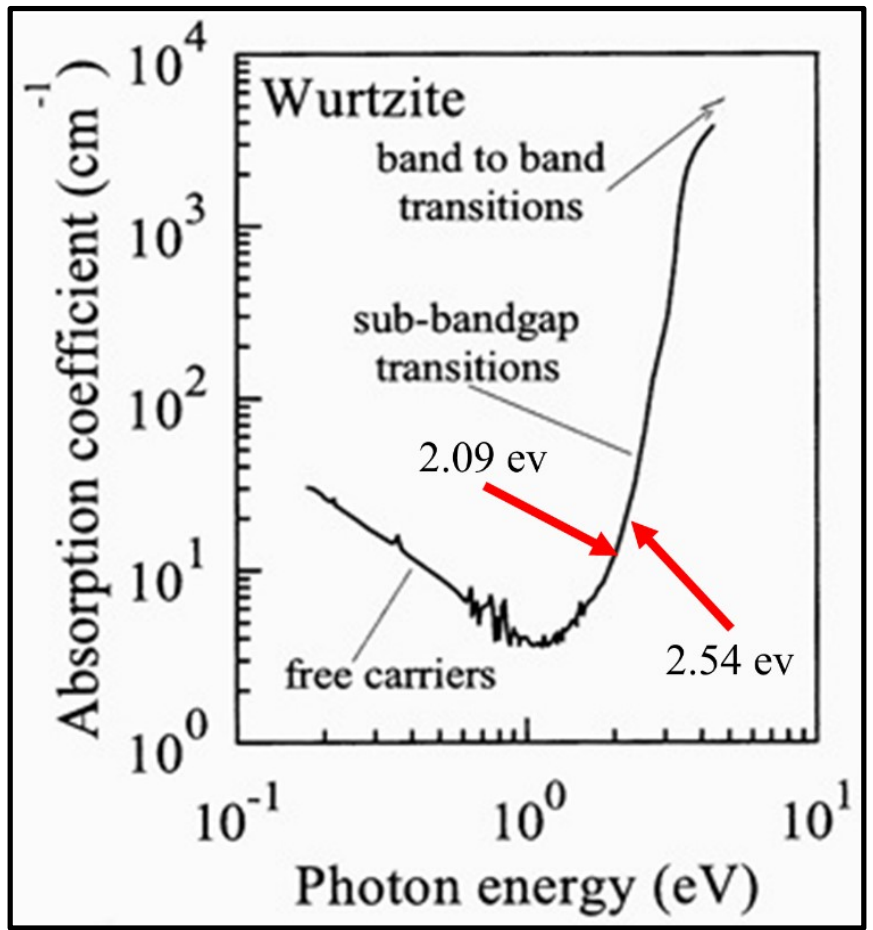

Figure 4.16: Absorption coefficient of GaN [23]

We can see from Figure 4.16 that $\mathrm{GaN}$ has absorption edge starting at $\sim 1 \mathrm{eV}$. The

figure suggests that we will not be looking at band to band transitions, but rather

transitions that could be due to defects in the GaN. Since we are using $2.09 \mathrm{eV}$ and 2.54

$\mathrm{eV}$, we are not seeing band to band transitions near the bandgap at $3.4 \mathrm{eV}$. Since $\mathrm{CPC}$

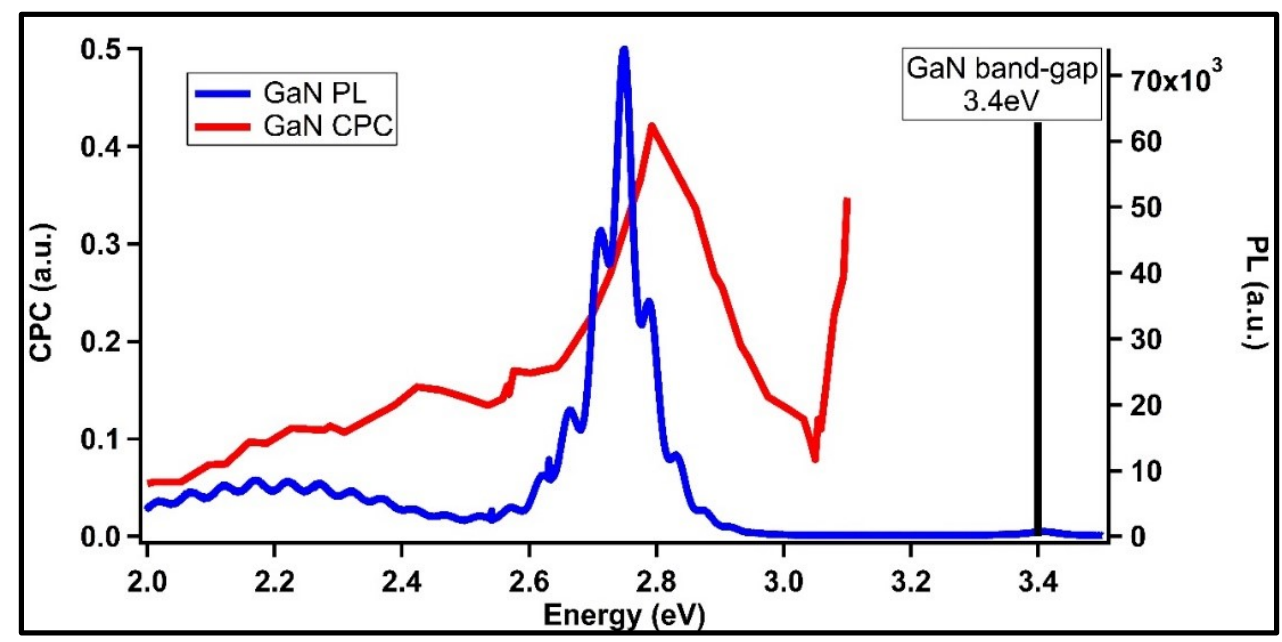

Figure 4.17: Comparison of GaN photoluminescence and CPC spectrum. 
depends on a sample's absorption and the STED system has both $2.09 \mathrm{eV}$ and $2.54 \mathrm{eV}$ beams available, either energy is within GaN's absorption. Therefore, GaN is a good option for scanning-CPC images. We then took a CPC spectrum of GaN by mounting it in the standard configuration shown in Figure 2.16, using the OPA system as the light source and maintaining the laser power at $5 \mathrm{~mW}$ for each energy with a $1 \mathrm{kHz}$ repetition rate. The red trace in Figure 4.17 is the $\mathrm{CPC}$ signal of $\mathrm{GaN}$, showing that $\mathrm{GaN}$ has a usable CPC at both $2.09 \mathrm{eV}$ and $2.54 \mathrm{eV}$. In Figure 4.17, we also have the photoluminescence (PL) data (blue trace). We see that the PL and CPC spectrums are shifted, implying that the mechanisms for each process are different. The PL data shows the traditionally seen 'yellow luminescence' at $\sim 2.0-2.5 \mathrm{eV}$ and a shifted near-band edge peak at $2.6 \mathrm{eV}-2.7 \mathrm{eV}$. The yellow luminescence is attributed to donor-acceptor pair (DAP) recombination due to the crystal surface or crystal interfaces within the substrate [34]. Yet, we see that the CPC signal does not line up to the PL data This misalignment could be because CPC depends on electron-hole separation rather than emission of sample as PL requires.

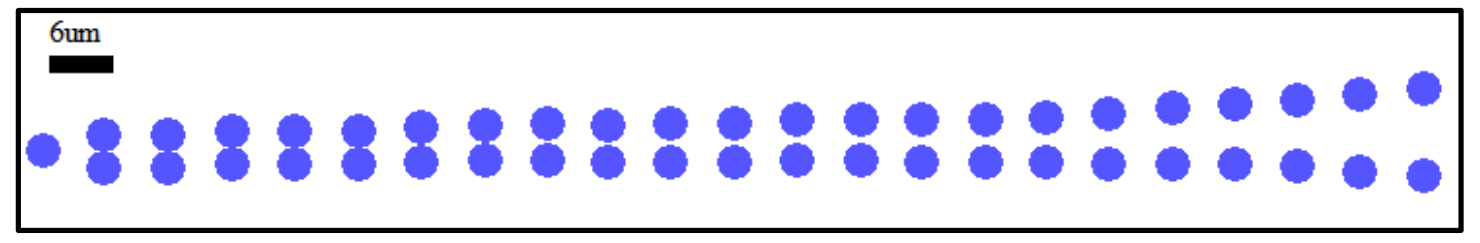

Figure 4.18: Masked used for resolution testing pillars.

Using a standard semiconductor wafer also provides the opportunity to fabricate custom geometries and features for testing the scanning-CPC limits. We designed studies on pits etched into the GaN wafers as well as a variety of pillars. For the resolution testing GaN pillars, we clean the wafers with $\mathrm{HCl}$, acetone, IPA, and rinsed in DI water. A chromium/nickel mask is applied with the pillar pattern. Then, the wafer is etched in a 
ICP (inductively coupled plasma) system at $400 \mathrm{~W}$ with the RIE (reactive-ion etch) at $60 \mathrm{~W}$ a chlorine flow rate of $14 \mathrm{sccm}$ (standard cubic centimeter per minute), and an argon flow rate of $6 \mathrm{sccm}$. The e-beam lithography was done at the University of Minnesota using the mask with the pattern shown in Figure 4.18. This fabrication process provided us with 3 um pillars varying in separation from $50 \mathrm{~nm}$ to $5 \mathrm{um}$. Sowmya Kolli, in the University of Louisville cleanroom, performed a similar procedure to produce

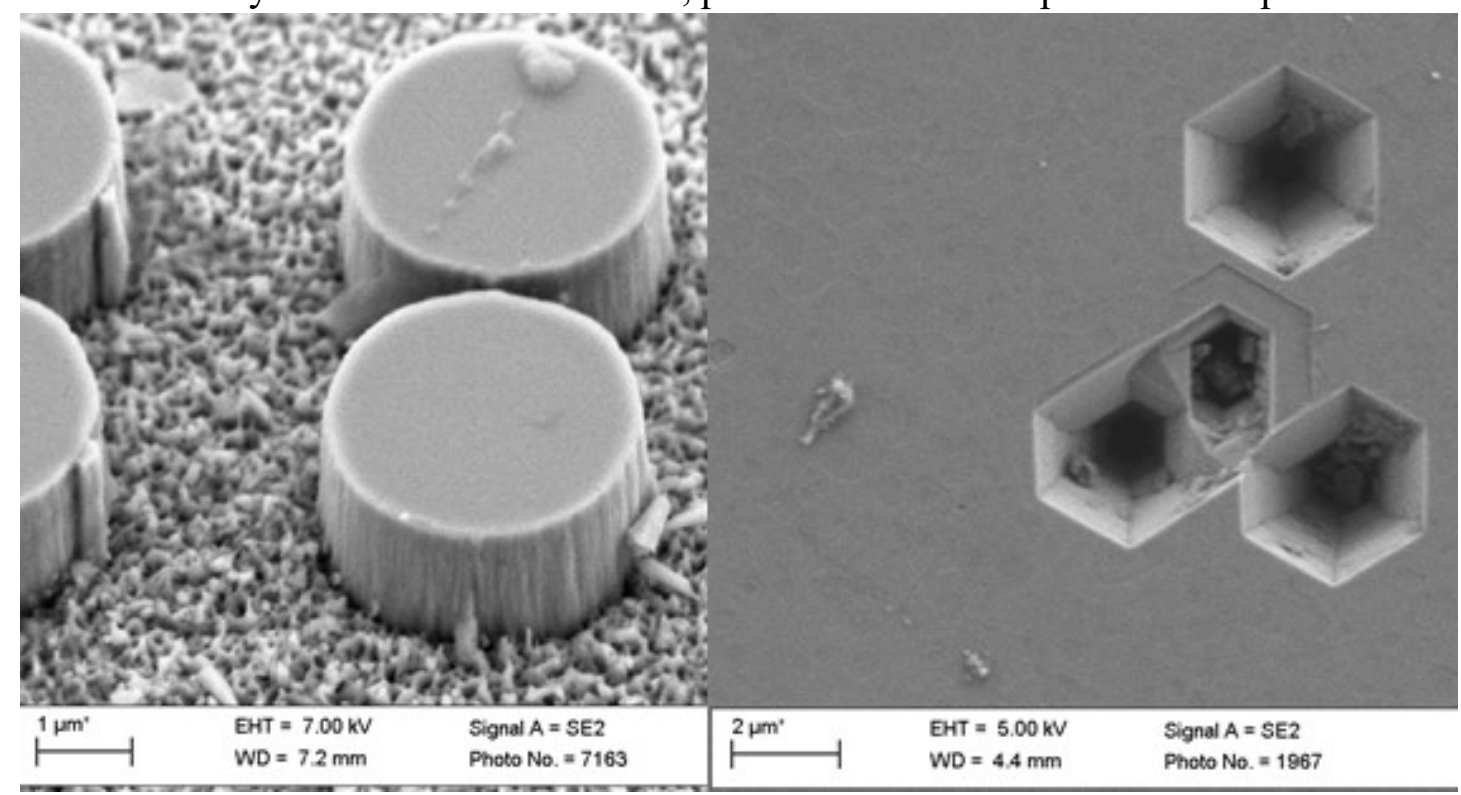

Figure 4.19: SEM of GaN pillars (left) and pits (right).

equidistant pillars as well as larger diameter pillars for preliminary testing. The pits are created with a simple timed phosphoric acid etch by submerging the wafer for up to 600 seconds. The pits were the initial focus of our scans since the fabrication procedure was quicker and simpler than that of the pillars. The pits made up a majority of our early scans, followed by the larger diameter pillars, and then finally looking at the resolution testing pillar layout. In Figure 4.19, we can see SEM images of the results of fabrications 3 um pillars and etching $\sim 4 \mathrm{um}$ pits in $\mathrm{GaN}$ wafers. 


\subsubsection{Preliminary GaN scans}
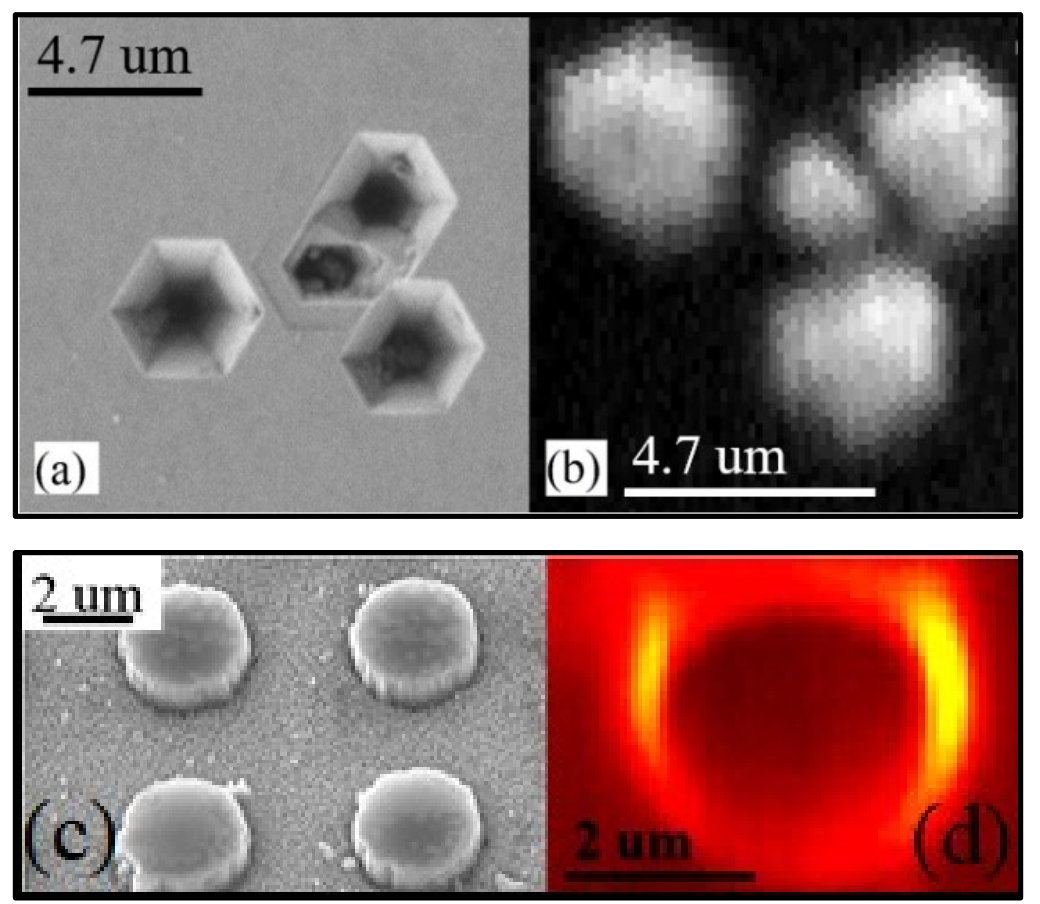

Figure 4.20: SEM (a) and CPC (b) image of shallow GaN pits and pillars SEM (b), CPC (c).

Using the scanning-CPC setup shown in Figure 4.1, we captured the CPC images

shown in Figure 4.20, where (a) shows SEM images of the pits with the CPC image shown in (b), similarly the SEM of the pillars (c) can be compared to the CPC image seen in (d). These images lead to further questions, such as, why do we see signal differences at all in the GaN fabricated samples? If the samples are from a uniform material, bulk $\mathrm{GaN}$ in this case, the signal should be uniform across the entire sample, independent of topology. Yet, we see strong signals along the circumference of the pillars and along the interior of the etched pits. These images also show asymmetries in signal distribution on the features, seen most readily in Figure 4.20 (d). Seeing a signal implies that the perimeter of the pillars and the slopes of the pits have features that either increased 
absorption or increase charge separation. Likewise, the asymmetry means that those segments have further increase absorption or charge separation.

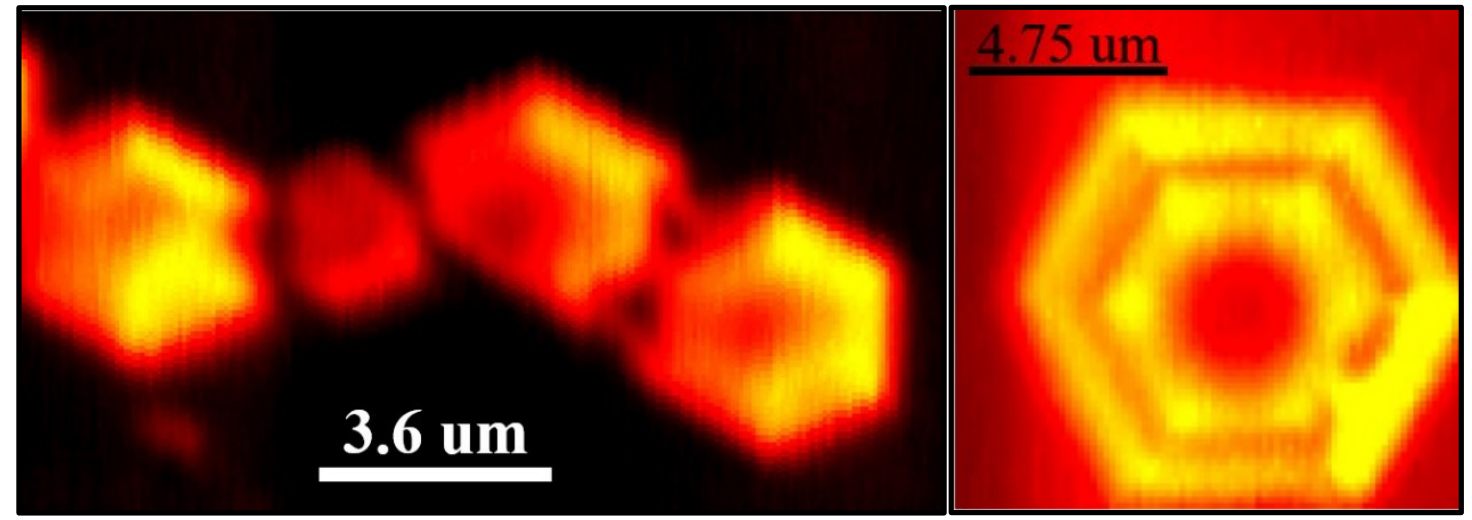

Figure 4.21: Short etch time (shallow) versus long etch time (deep) GaN pits.

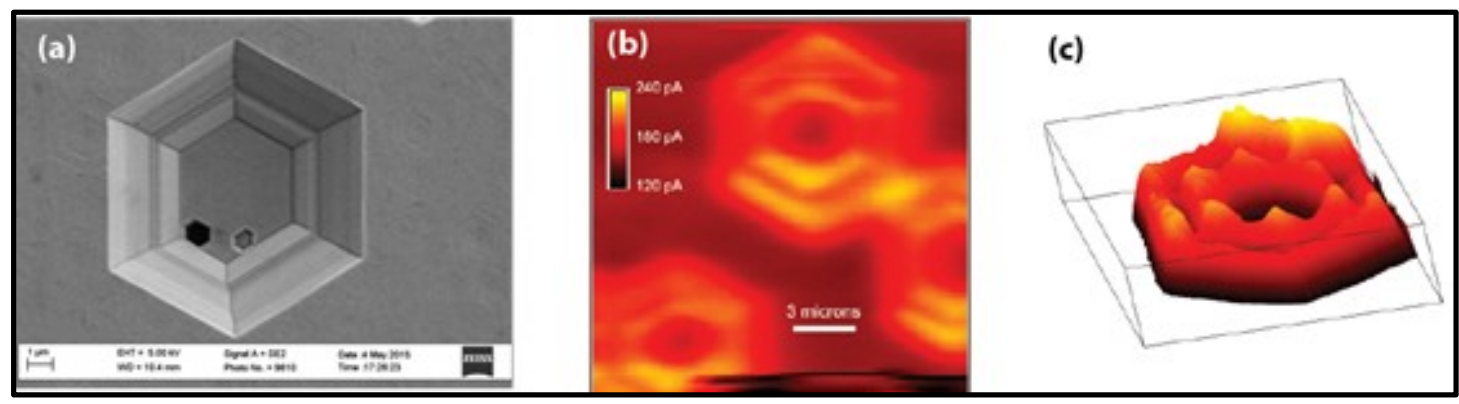

Figure 4.22: SEM (a), 2D CPC (b), and 3D CPC of a deep GaN pit.

If the CPC signal depends on these edges in some way, then we can test this dependence directly by changing the fabrication parameters. This change is most easily done with the GaN pits, since it only involves etching the wafer longer to create substantially distinctive features. Figure 4.21 also shows the difference in short (left) versus long (right) etch times for the GaN pits. We see that the longer etch time pits are almost twice as wide and feature secondary signals within their perimeter. We can also see how the shorter timed pits, like those in the SEM in Figure 4.20, do not bottom out to the sapphire substrate beneath, where the longer etch time pits are much deeper and do bottom out as shown in the SEM in Figure 4.22 (a). Figure 4.22 also corroborates the idea 
of a secondary structure in the longer etch time pits, where the SEM shows terraces before reaching the sapphire base substrate.

Since the CPC signal depends on both the absorption of photons by the sample and the degree to which the electron and holes separate to generate an electric field, what about these geometries causes the signal? It is possible that the areas of high signal are also areas of increased defect density, or that areas of higher defect density are exposed. It could also be that the etched areas have less microscopic debris, allowing for more direct absorption. It is possible that a piezoelectric effect, generated by strain in the walls of the pits or pillars induces an electric field within the sample and enhances the CPC signal. These questions led us to testing the hypotheses that absorptions and election-hole separation changes caused the areas of high CPC signal, indicating that the high signal regions are places where absorption is increased, possibly due to defect states, or that these high signal areas have increase electric field activity, possibly caused by a piezoelectric feature of the GaN.

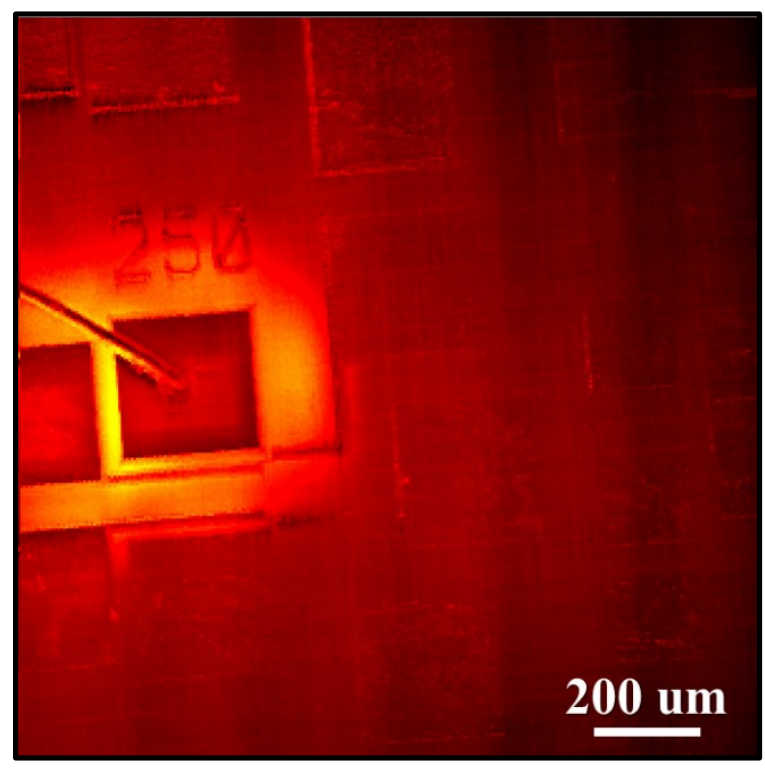

Figure 4.23: Wide area scan of GaN cantilever 


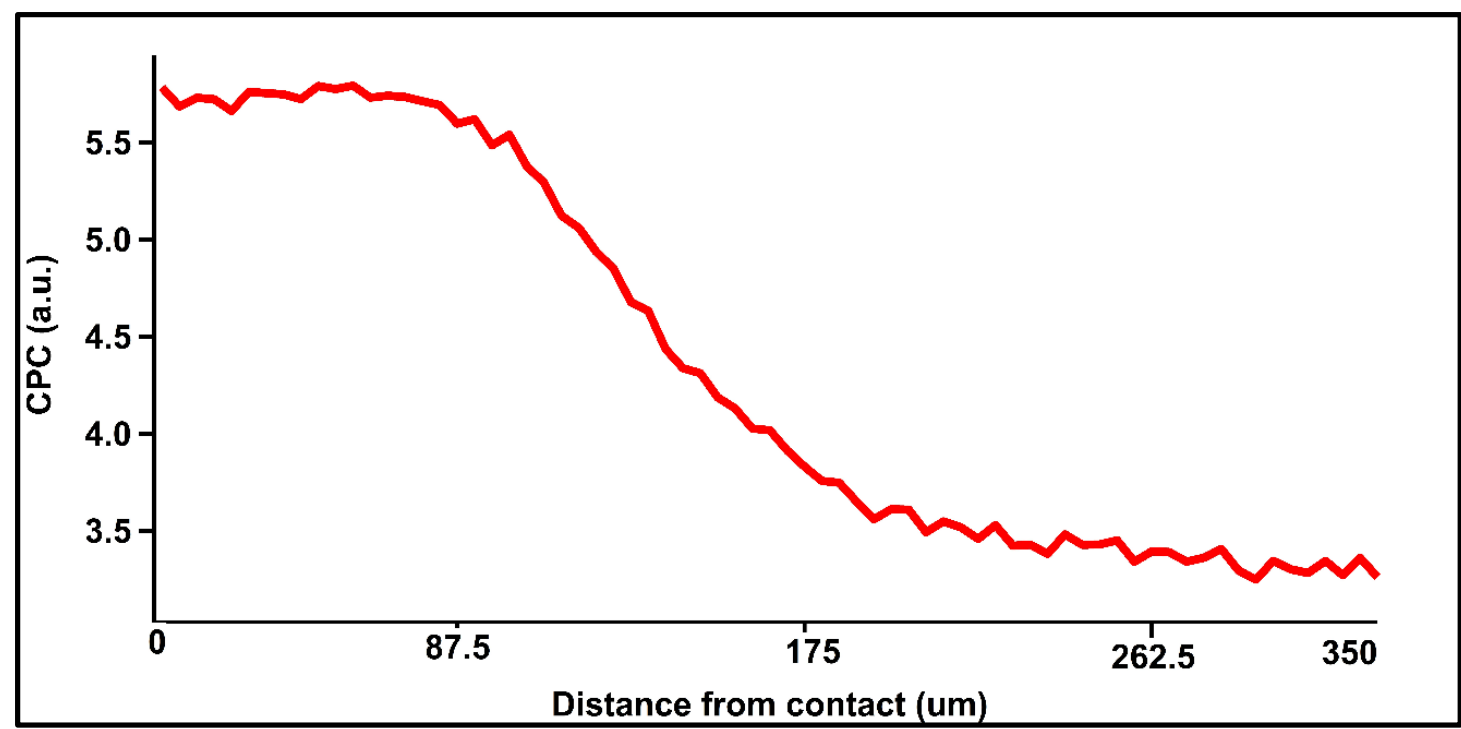

Figure 4.24: Line scan of CPC signal from edge of contact to 350um away from contact.

We must then investigate what effect the position of the contact has on the CPC signal. Figure 4.23 shows that the signal decreases with respect to the distance from the contact. A line scan from the edge of the contact to $\sim 350 \mathrm{um}$ is shown in Figure 4.24. We see that the signal is relatively consistent within 100 um of the contact with a sharp decrease after that point until the signal reaches the same range as the noise. This result means that the location, formation, and design of the contact has a direct effect on the CPC signal. The same result is also an indication of why we have some of the asymmetries seen in the GaN pillars and pits. The asymmetries could be due to a contact placement or design effect.

\subsubsection{Testing electric field effects}

As we described in the CPC discussion, the measurement is a combination of photon absorption and electron-hole separation. We must look at the effect of electric fields in $\mathrm{GaN}$ on the resulting CPC signal. We made use of simulations done by Pranoy Deb Shuvra, of the piezoelectric properties of the GaN pillars. This simulation, shown in 
Figure 4.25, was done using the finite element model (FEM) in COMSOL simulating the GaN pillar. COMSOL applies the properties of the GaN wafer to this pillar element and then generates a plot of the expected electric field in the pillar and in the surrounding area.

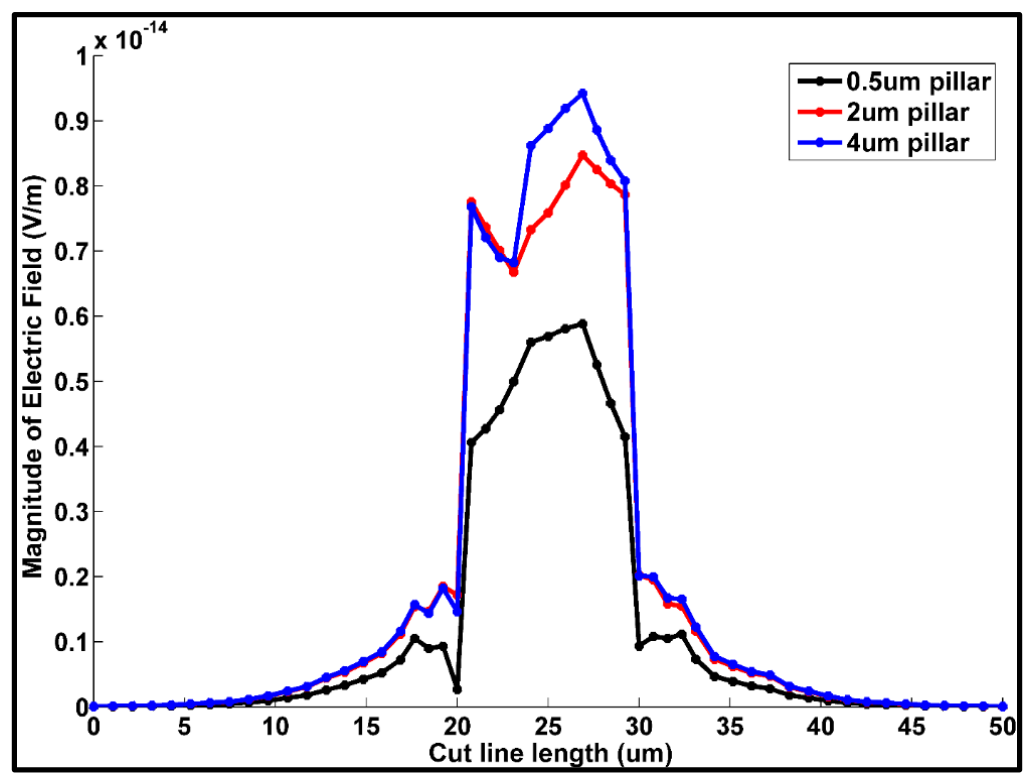

Figure 4.25: GaN 10um pillar piezoelectric electric field

Figure 4.25 shows the calculated electric field around 10 um diameter GaN pillars that are $0.5,2$, and 4 um tall. This simulation, showing the highest electric field at the tops of the pillar, did not match our initial thought that the signal seen on the edges of the GaN pillars and pits was due to a piezoelectric effect from the crystal structure within the material. This simulation means that if we were seeing a piezoelectric effect then the CPC signal should be maximized at the tops of the pillar, not only the edges of the pillars.

We also tested the piezoelectric-enhancing hypothesis experimentally in silicon samples. We had a member of our group, Pranoy Deb Shuvra, testing silicon cantilever designs. In Figure 4.26, we have a cross sections of the cantilever fabrication process: base substrate seen in (a), with an $\mathrm{Al}$ deposition (b), then etching the $\mathrm{Al}$ to form a contact 


\begin{tabular}{|c|}
\hline (100) Silicon \\
\hline Silicon \\
\hline
\end{tabular}

(a)

Pattern aluminum to form contact pads

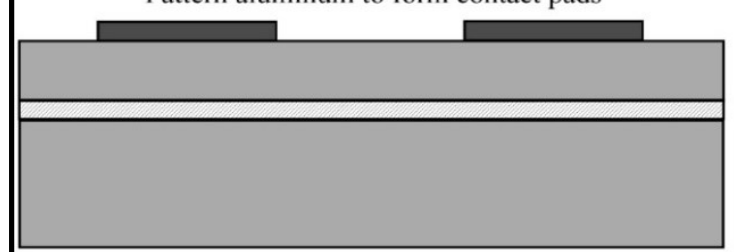

(c)

Backside DRIE to open release window

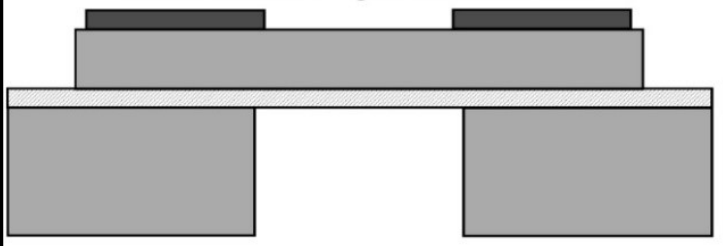

(e)

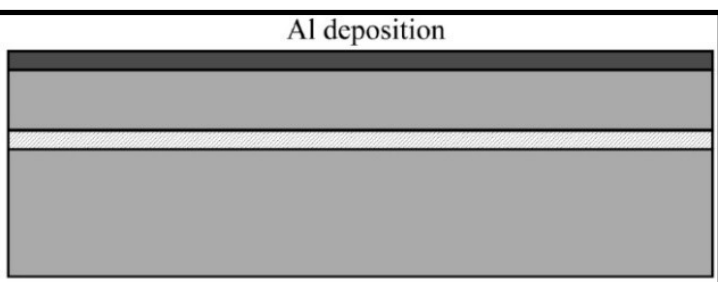

(b)

Front-side DRIE to define piezoresistor

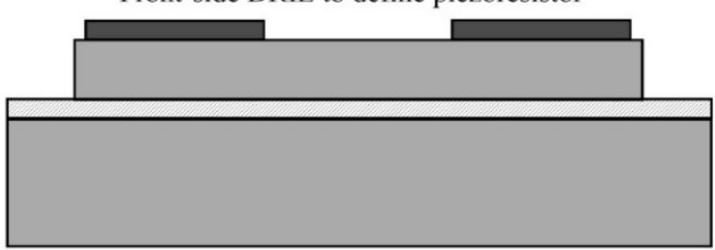

(d)

Dry etch $\mathrm{SiO}_{2}$ to release the piezoresistor

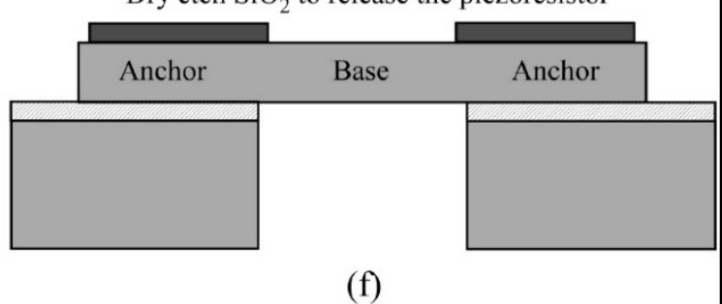

Figure 4.26: Fabrication of the Si cantilevers [1].

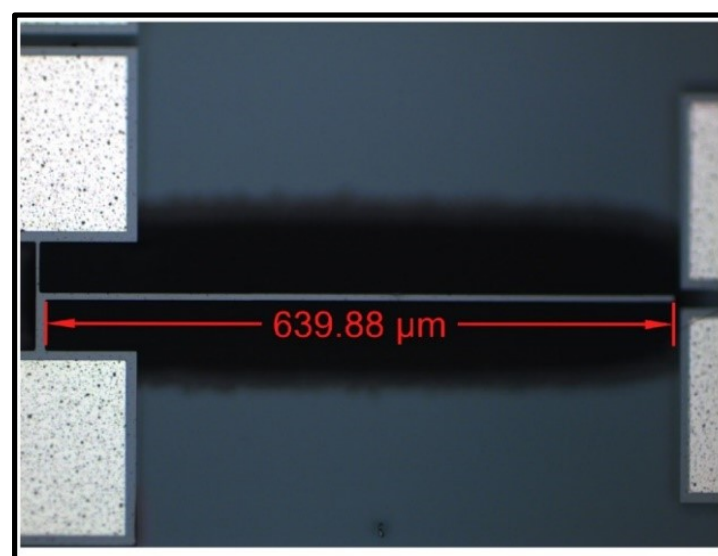

(a)

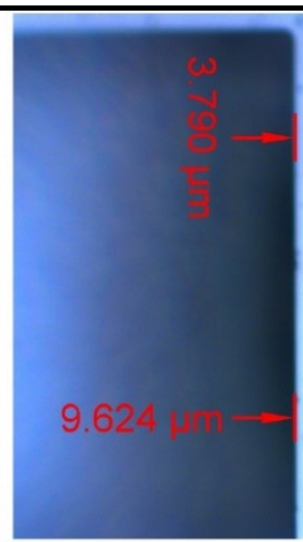

(b)

Figure 4.27: Top down photo of $\mathrm{Si}$ (a) wide view and (b) close up. [1] (c), the top silicon is the removed via a Deep Reactive-Ion Etch (DRIE) (d), the bottom of the beam is opened with another DRIE (e), and finally a dry etch removes the layer of $\mathrm{SiO}_{2}$ along the bottom of the cantilever(f). This process creates a free-floating cantilever with free floating supports to either side. This structure provides us with an easy method for testing the change in the CPC signal when an internal electric field is induced by 
straining a piezoelectric material. Figure 4.27 shows a top down view of the $\mathrm{Si}$

cantilevers when in the scanning-CPC system. We placed a micro-positioning probe on the stage to move with the sample. This probe then pushed the cantilever side to side (rather than the traditional up or down) to generate strain along the support beams of the cantilever. The probe applying the strain was electrically isolated and its holder insulated so that is did not generate any changes in the CPC signal.

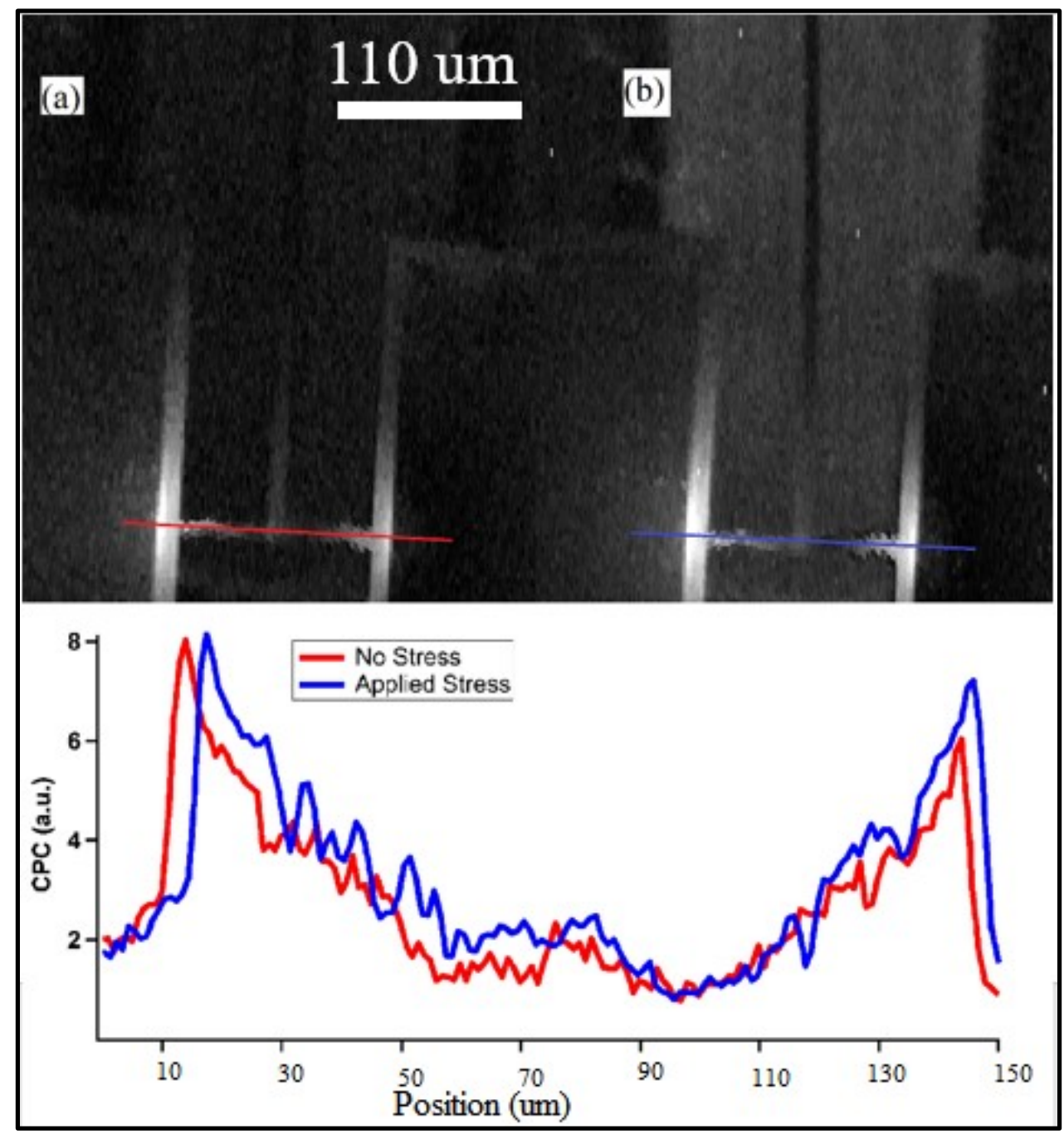

Figure 4.28: Effect of stress on CPC signal in a silicon cantilever.

We compared the unstrained cantilever, Figure 4.28 (a), with a cantilever that has been pushed left by $\sim 25 \mathrm{um}(\mathrm{b})$. Then line scans can be done across the supports of the 
cantilever, showing in Figure 4.28 (c) the applied stress (blue trace) does not create a significant difference in the $\mathrm{CPC}$ response when compared to the no stress line scan (red trace). This result is further evidence to show that the CPC signal is not a result of, nor is the signal significantly modified by, piezoelectric properties of semiconductor samples.

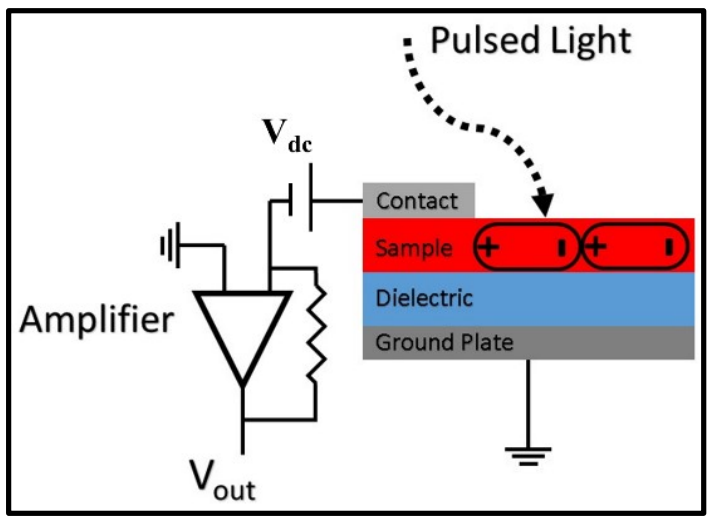

Figure 4.29: Diagram of bias testing GaN samples.

We also looked at the effects of an externally applied electric field. We applied a DC voltage to the sample by simply adding a power supply in line with the contact as shown in Figure 4.29. We then did line scans across 5um diameter pillars. We can see from Figure 4.30 the effects of applying a bias across the sample where the black trace is a line scan without any biasing, the red trace is a line scan with significant positive

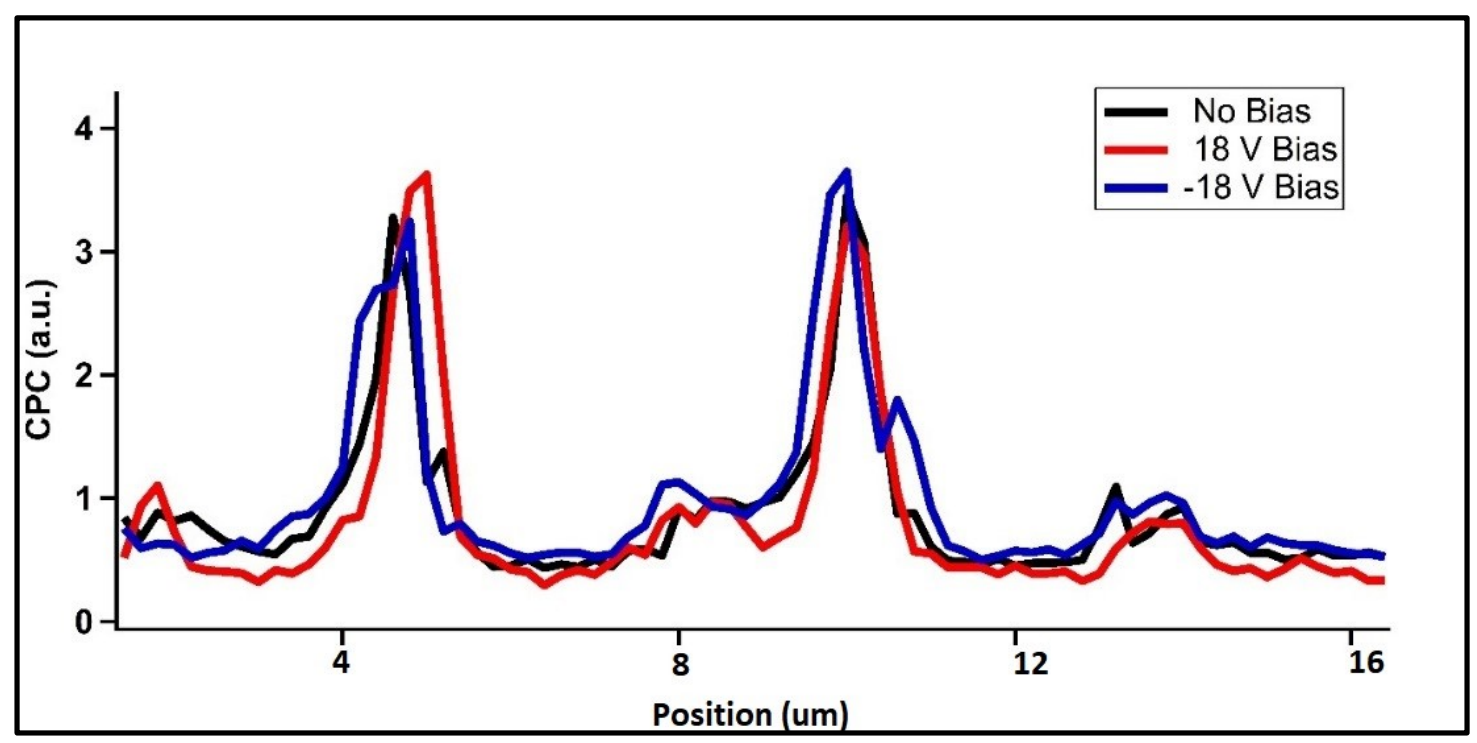

Figure 4.30: Effects of external bias on CPC signal of GaN pillars 
biasing $(18 \mathrm{~V})$, and the blue trace is a line scan with a significant negative bias $(-18 \mathrm{~V})$. We know from the work described earlier, in Chapter 2.3, that biasing should have a significant effect on the CPC signal of a sample if the CPC signal is due to similar effects like those seen in Chapter 2.3. That means that if the signal seen on the perimeters of the pillars is caused or enhanced by an undetermined electric field effect then the bias would either increase of decrease the signal strength. Yet, we do not see strong changes in the amplitudes of the CPC signal. This result further implies that the CPC signal we see is not due to electric field properties of GaN.

These results indicate that the CPC signal seen in GaN features is not a function of the electric field showing that it is not dependent on some internal electric field or easily manipulated by an external electric field. This relative independence means that we must look to other effects for the source of the CPC signal. In the next section, we will discuss how absorption changes affect the CPC signal.

\subsubsection{Testing absorption effects}

Testing the absorption hypothesis was the most straightforward by examining the material and setup properties: GaN defect density, crystal structure, surface quality, and contact arrangement, since they could all determine both the signal strength and the CPC image properties.

We fabricated pillars in GaN wafers so that we could study why the walls of the pillars gave use such a strong CPC signal. It could be that the exposed walls allow for subsurface defects to be exposed to light, increasing the CPC signal. We fabricated two sets of pillars on two different GaN wafers, with one wafer having a higher defect density 
that the other wafer. We can verify the difference in defect density by looking at the I/V curves taken from the devices fabricated by Sowmya Kolli, shown in Figure 4.31. Sample 1 (red trace) shows a much lower breakdown voltage than Sample 2 (blue trace), showing that Sample 2 does have a lower defect density [35].

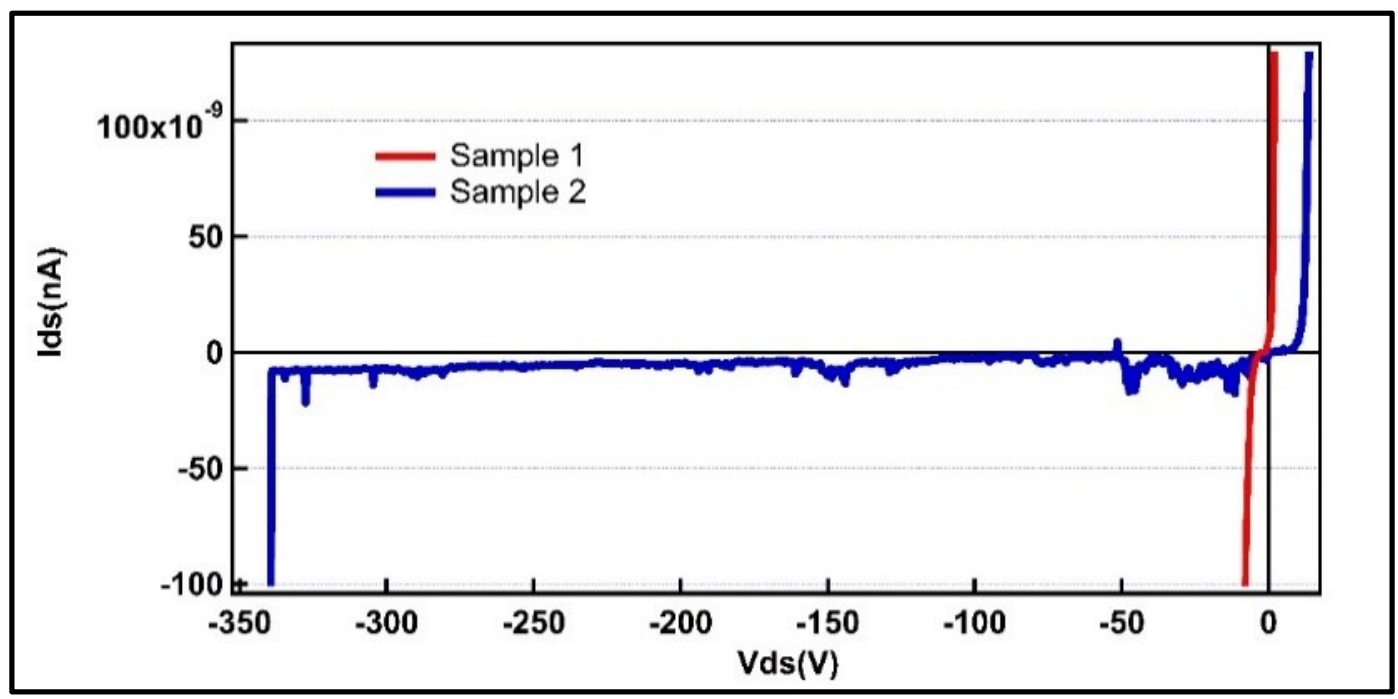

Figure 4.31: IV curves of diodes made from Sample 1 and Sample 2.

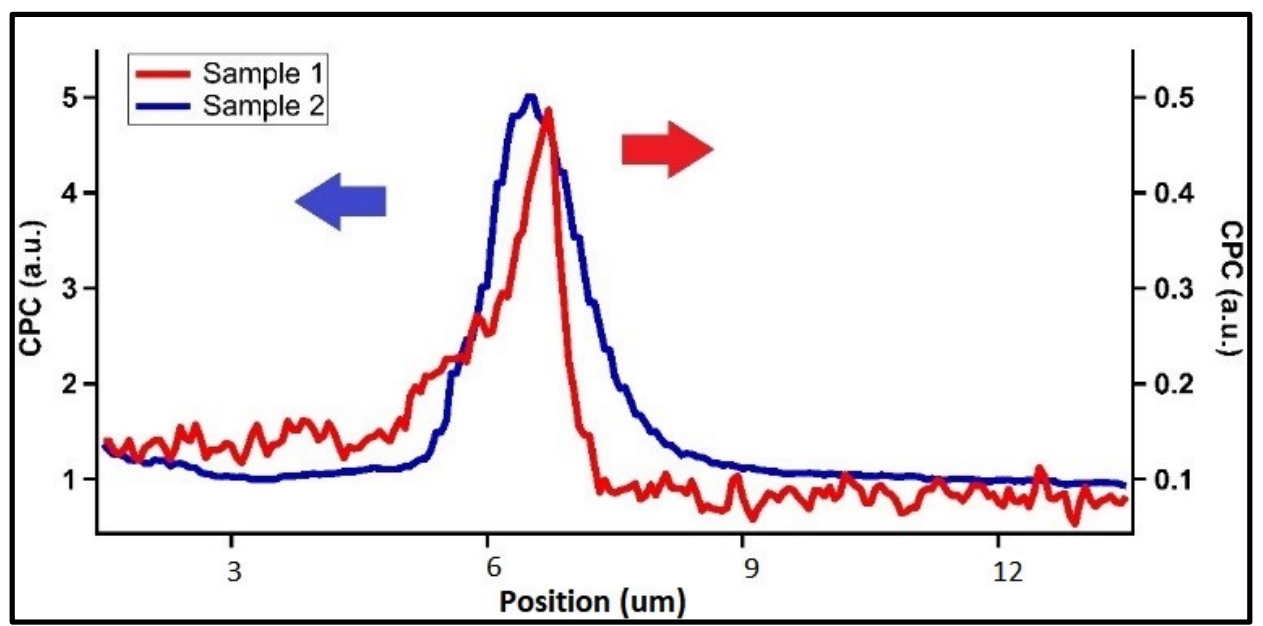

Figure 4.32: CPC of pillar edge between different defect densities

We tested if scanning-CPC measures defect densities, since a sample with higher defect densities would have more CPC signal due to more exposed defects. Figure 4.32 shows the line scans of the high defect GaN wafer, Sample 1 (red trace), and the lower defect density sample, Sample 2 (blue trace), note that the scale for Sample 1 (red trace) 
is a tenth of that of Sample 2. We see that the high defect sample has a significantly lower signal, $\sim 10 \mathrm{x}$ lower. This result implies that increase in CPC signal is not due to an increase in defects, nor does the signal depend on defects on the edges of the pillars in the manner we predicted. The relationship between defect density and CPC signal seems inversely related. With decreasing defect density, we see an increase in the CPC signal. Since the CPC signal depends in part on absorbance of the sample, this implies that Sample 2 has better absorbance qualities.

A second consideration is how the fabricated designs affect the signal. In Figure 4.33, we have a pair of pillars with different etch times. The left pillar has a 600 second etch and the right side is a 300 second etch time. The line scans show an expected result, a longer etch time creates a taller pillar, providing a larger surface for photon absorption to take place, therefore the 600 second etch time pillar has a higher CPC signal. This result also shows that the asymmetry ratio increases in the 600 second etch time, implying that the asymmetry is more than just a factor of direct, surface only absorption.

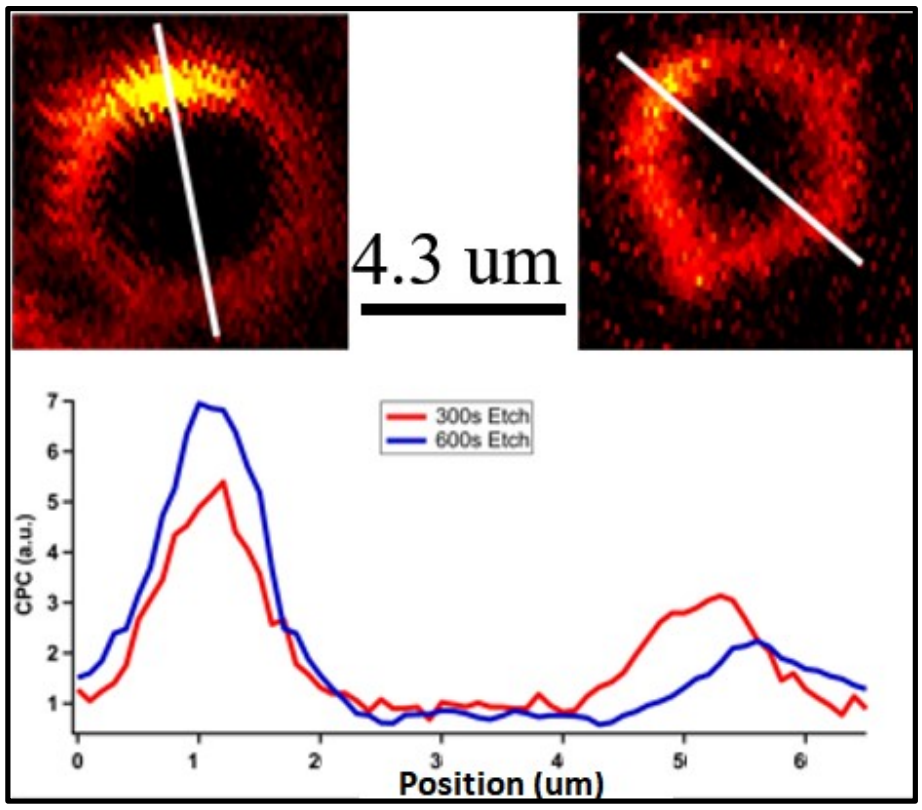

Figure 4.33: Etched time comparisons, tall versus short pillars. 
If it did rely only on surface absorption, then we should see uniform increases in the signal all around, rather than an overall increase in signal, and also an increase in the ratio of high side to low side.

The next possible parameter that determines a samples CPC response is its surface quality. In the GaN samples, this can be controlled in a variety of ways. The etching process does not leave a pristine surface. By not cleaning the surface after etching, we have a 'dirty' sample we can compare with a 'clean' sample, where the cleaning process is treating the sample with $\mathrm{HCl}: \mathrm{H}_{2} \mathrm{O}$, Acetone, IPA, then DI water. A second method of modifying the surface of the $\mathrm{GaN}$ samples is through sulfur passivation. By soaking the etched $\mathrm{GaN}$ wafers in sulfuric acid, the surface of the sample has less surface charge available due to the passivation process reducing the charge density on the surface through an oxidization process [36].

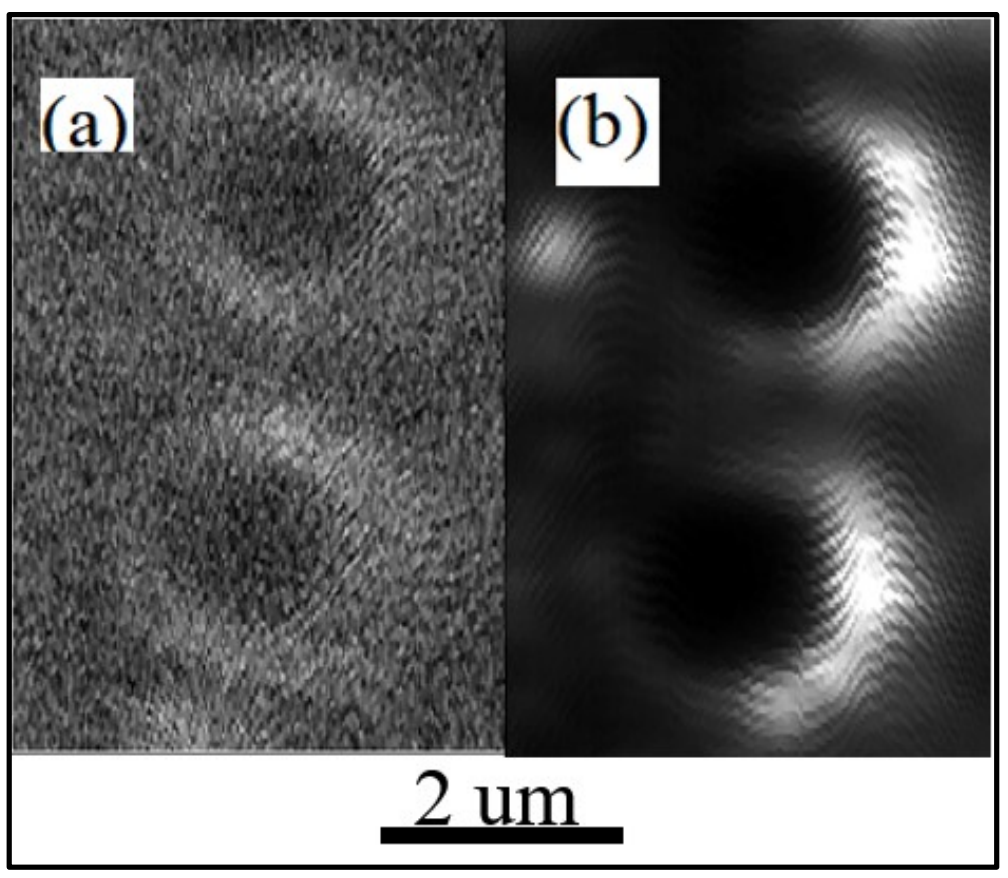

Figure 4.34: Dirty (a) versus clean (b) GaN pillar

In Figure 4.34, we have a comparison between a dirty and a clean sample. This figure shows a significant signal difference. The samples are identical except for the 


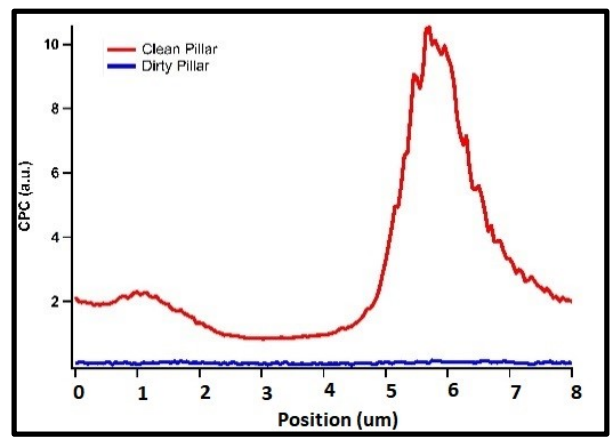

Figure 4.35: Line scans from the features seen in Figure 4.34.

cleaning procedure being applied. This increase in signal to noise ratio also results in an increase in the resolution of the image. This result shows that the surface quality is a significant factor in both signal generation and resolution in the CPC system. This result is shown in Figure 4.35 and the lines scans through the pillars shown in the Figure 4.34. With the line scans on the same y-axis scale, this direct comparison shows the strength of the change in the CPC signal. The system dirty pillar signal can look good on its own, but when compared to the clean sample's signal, we see that the effect is a dramatic improvement.

In Figure 4.36, we see the effects of passivation on a clean GaN pillar sample. Passivation improves the image quality and the signal to noise ratio. This comparison shows a significant improvement in resolution of the sample as well as signal strength. By looking again to line scans across these features, we get a better idea of the effects of passivation, as shown in Figure 4.37 where the red trace shows the CPC signal for a passivated pillar and the blue trace shows the CPC signal for an unpassivated pillar. Both pillars were cleaned prior to scanning and passivation. This comparison shows that by improving the surface of the sample, the resulting image is improved. Therefore, a higher quality surface where there are less opportunities for excited electron-hole pairs to 


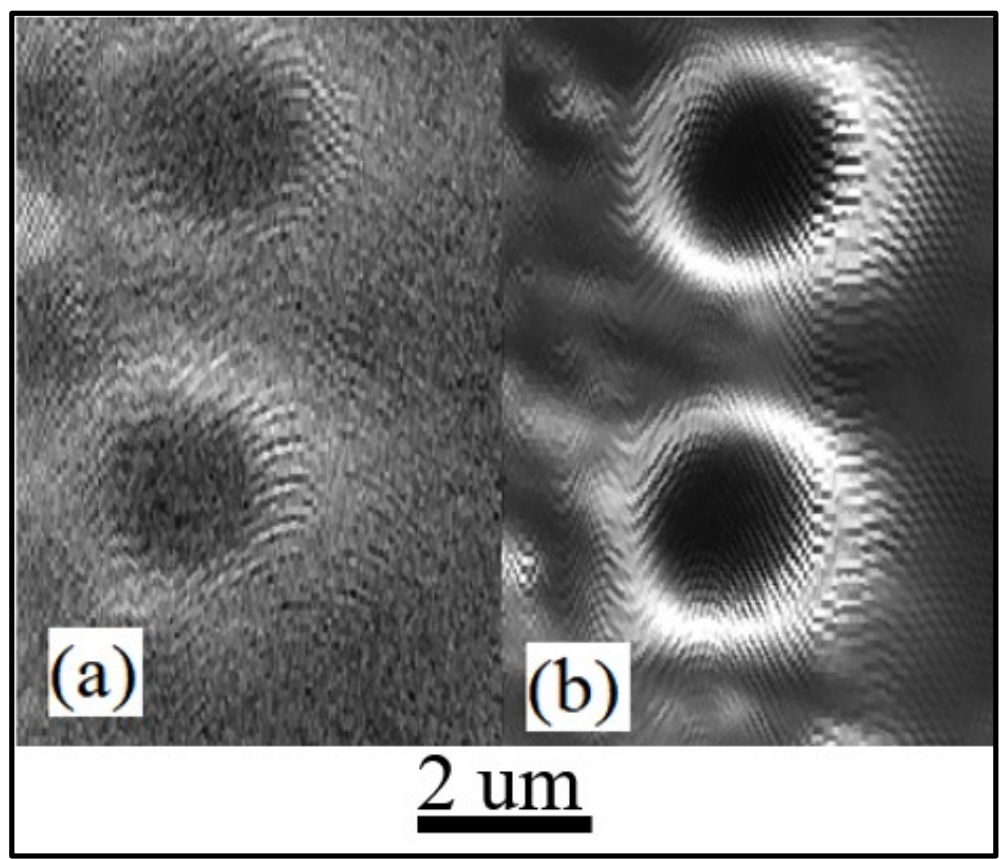

Figure 4.36: Unpassivated (a) versus passivated (b) GaN pillars

recombine will result in a higher CPC signal. This result matches with the ideal CPC

parameters: electron-hole pairs that separate some distance, generating an electric field.

Without the sulfur passivation, the electron-hole pairs either do not separate, equalize too rapidly to detect, or the light is not able to propagate further within the sample.

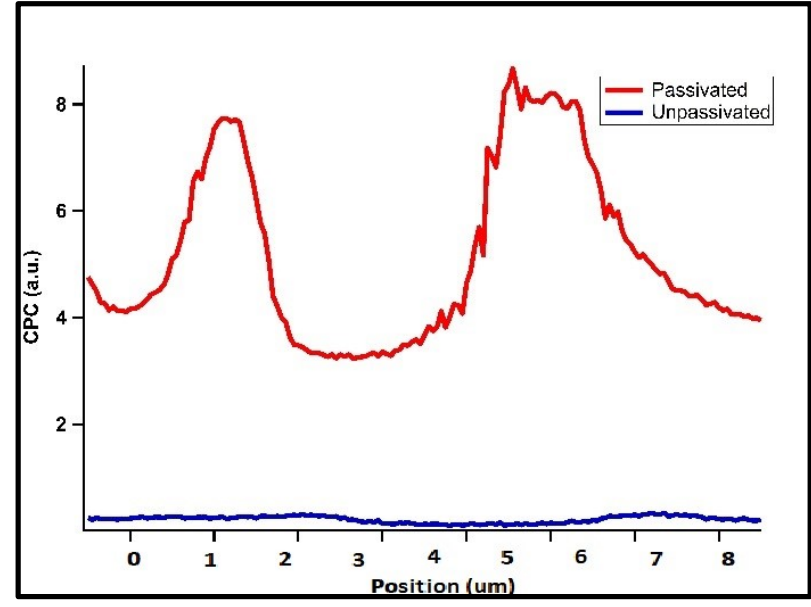

Figure 4.37: Line scans of the features seen in Figure 4.36.

We see that changes in the possible absorption parameters in the GaN dictate both signal strength and resolution. If a sample has a better surface quality, it produces a higher, clearer signal. The surface quality can be both a measure of topological 
obstructions (cleanliness) or electronics structure (passivation). This set of results means that we can tune samples to give us the best images possible with respect to enhancing absorption. We have also shown that the defects are not the primary source of the CPC signal by testing a sample with high defect density versus a low defect density sample. We have also shown that exposing a larger area of pillar wall does increase signal, but not as precise as we had expected. These results mean that there is a mechanism involved that we have not tested yet, leading to the hypothesis that the changes in the CPC signal and the asymmetry are due to a waveguiding effect within the GaN substrate. The next section will discuss the results of testing that hypothesis.

\subsection{Waveguiding effect}

A straightforward way to test if we are seeing a waveguiding effect is to look at the position of the contact with respect to the CPC signal and image profiles that result. We can test the effect of contact position by returning to the Si cantilevers since they have strongly delimited contact pad that we can precisely attach to and then changing the position of the contact used for data collection. Both contact pads are identical in the silicon cantilevers but the arms are different, one is thicker than the other. In Figure 4.38, (a) the top pad is used and in (b) the bottom contact pad is used for CPC signal collection. The most intriguing detail is that the highest signal regions on the cantilever is the side opposite the contact, which is counter intuitive since we would expect highest signal regions to be nearest to the contact. This result shows that the nearness to the contact does not exclusively influence the CPC signal leading to testing other contact configurations as well as the scanning parameters themselves. 


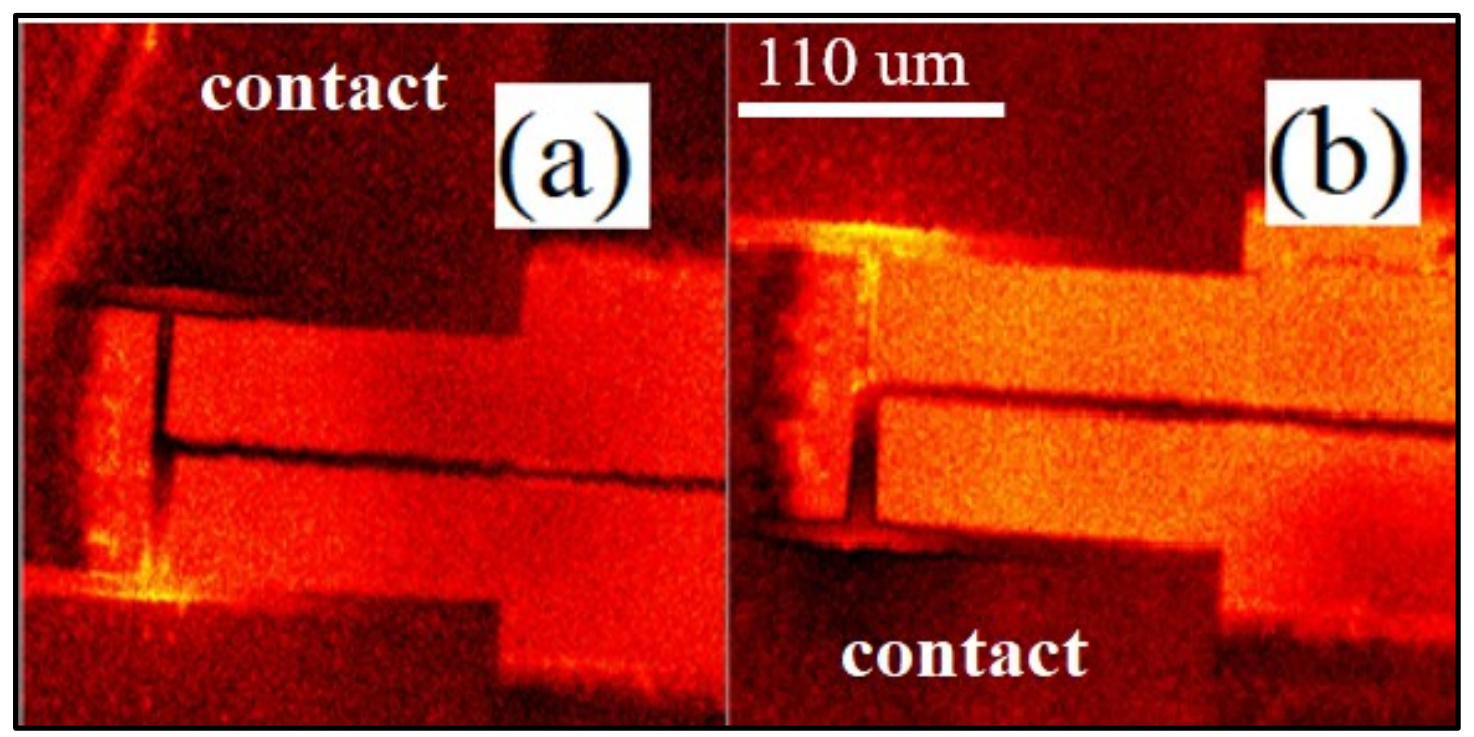

Figure 4.38: Top (a) and bottom (b) contact location on Silicon Cantilevers

We can test this on sided effect on the GaN pillars by 'painting' a circular contact around a scan area. In Figure 4.39, we have the area scanned (a) within the circular contact, (b) outside the circular contact, and (c) the wire connecting the sample to the BNC cable. The area outside the circular contact, (b), will behave in the same way as previous contacts.

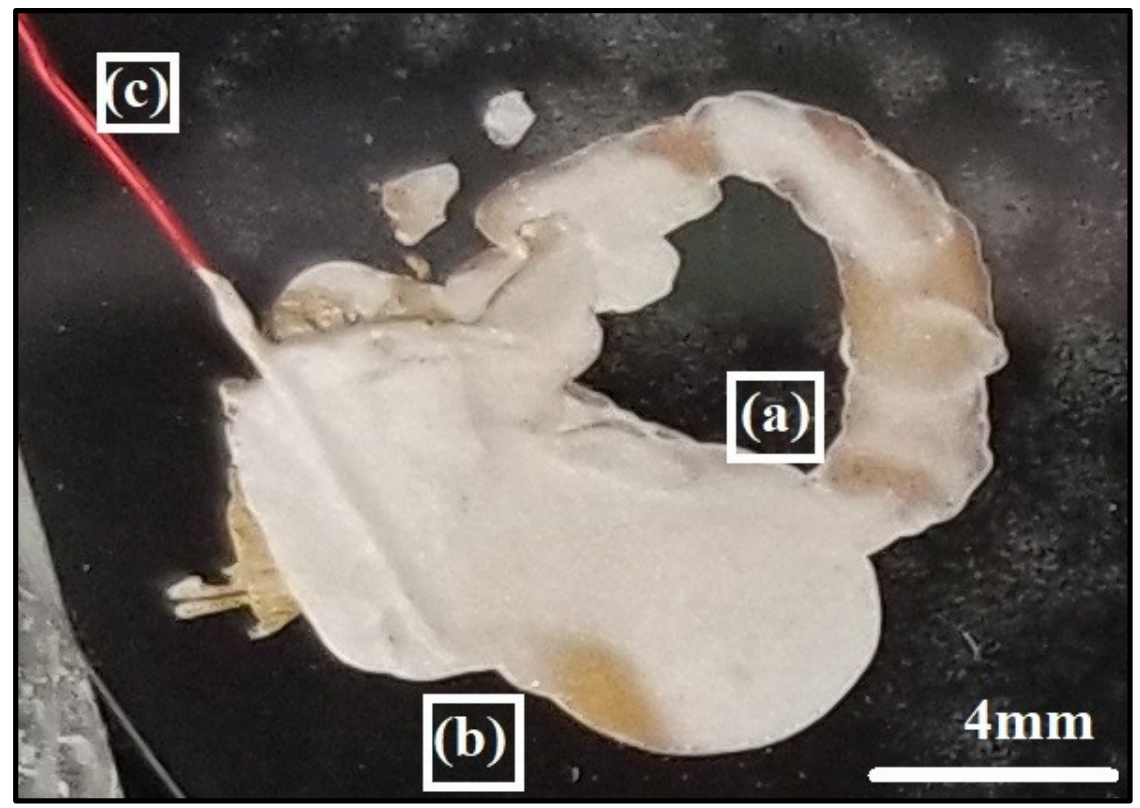

Figure 4.39: Photo of circular contact where images were taken (a) inside (b) outside, and the signal was collected via the contact (c). 
We can see this contact configuration dependence seen in the silicon cantilevers

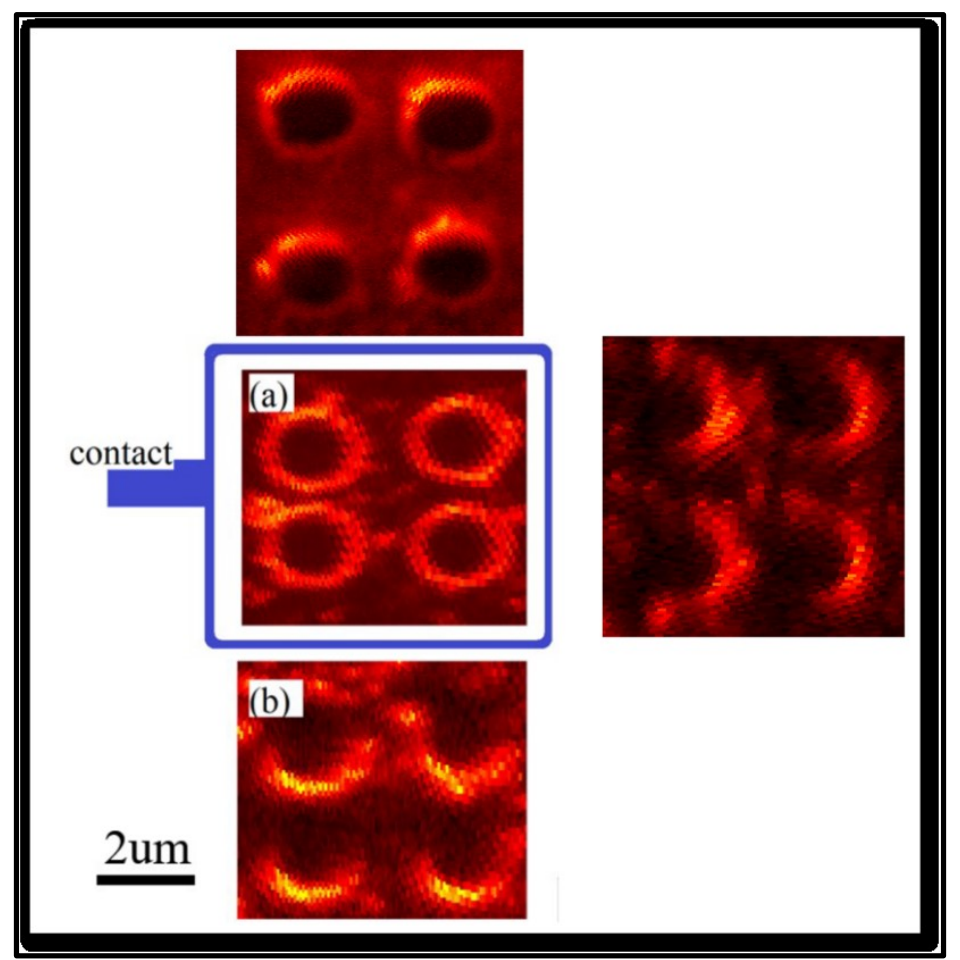

Figure 4.40: Pillar asymmetry seen in GaN pillars depends on position with respect to the contact.

in the GaN pillars too. Figure 4.40 shows two CPC scans and the conditions for those scans. Both scans maintained the same settings, both electrical and software, with only the location of the scan with respect to the contact changed. In Figure 4.40 (a), the scan was taken within a circular, silver paste contact where the blue trace represents the silver paste. In Figure 4.40 (b), the scan was taken below the contact showing that the contact directly effects the CPC signal, while in (b) we see the high signal region is opposite of the nearest contact edge, and (a) shows a more uniform signal response.

The best explanation of seeing a signal at all as well as seeing asymmetries in the $\mathrm{GaN}$ fabrications is that we are seeing a waveguiding effect. The waveguiding effect explains how the areas of highest signal are around the perimeter of the pillars of the slopes of the pits. These areas could have a longer beam path, increasing the signal due to 
increased opportunities for absorption therefore increased CPC signal. We can see the possible effect of total internal reflection within the GaN samples. In Figure 4.41, we see the beam path reflecting within $\mathrm{GaN}$, with the pit angle at $\theta=\sim 70^{\circ}$ as determined from the AFM measurements.

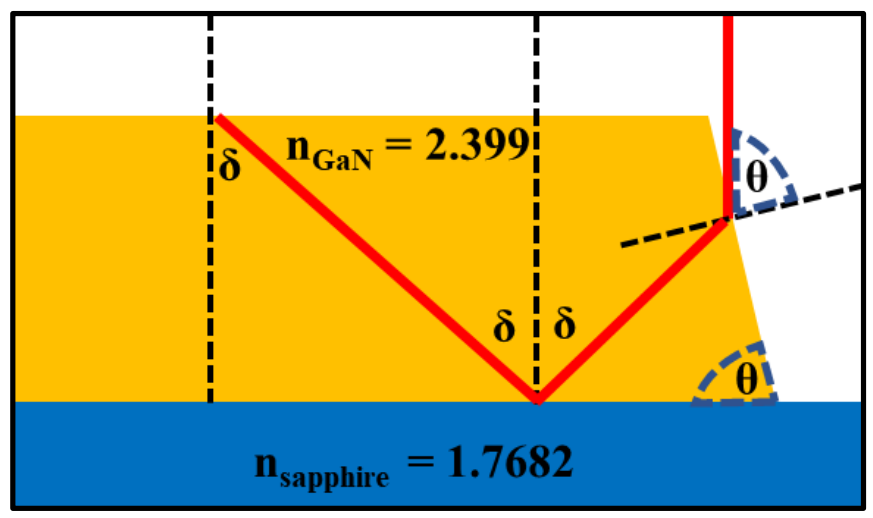

Figure 4.41: Possible total internal reflection in GaN

Figure 4.41 shows how a perpendicular beam would interact with the GaN surface. In our system, we use an Olympus UIS MPlanIR 100x /0.80 objective with a $180 \mathrm{~mm}$ focal length [37]. Knowing these parameters and the indices of refraction of GaN $(n=2.399)$ and sapphire $(n=1.7682)$ back along with that of STP air $(n=1.00029)$, we can simulate the effects of the oblique component of the beam. In Figure 4.42, we used RayOptics see the how along the walls of the pillars and pits the path length of the beam becomes much longer [16]. We see that in both the bulk of the pit and pillar areas, (a) and (c), the light propagates through the sample without increasing the beam paths. Yet, along the walls of the pits or pillars, (b) and (d), we have a significant increase in the length of the beam paths. We can even see that in the case of the pillar the light propagates significantly to the right. If the contact was there, more signal would be generated near the contact further increasing the CPC signal. With the increased path length of the light, more of the material can be excited thereby generating more electron-hole pairs and 
increasing the $\mathrm{CPC}$ signal. This signal due to a waveguiding effect, means that the $2 \mathrm{D}$ CPC image in $\mathrm{GaN}$ is not a local measurement, and it depends on where and how the light interacts with the surface.

This waveguiding hypothesis can also explain the earlier tests. With a decreased

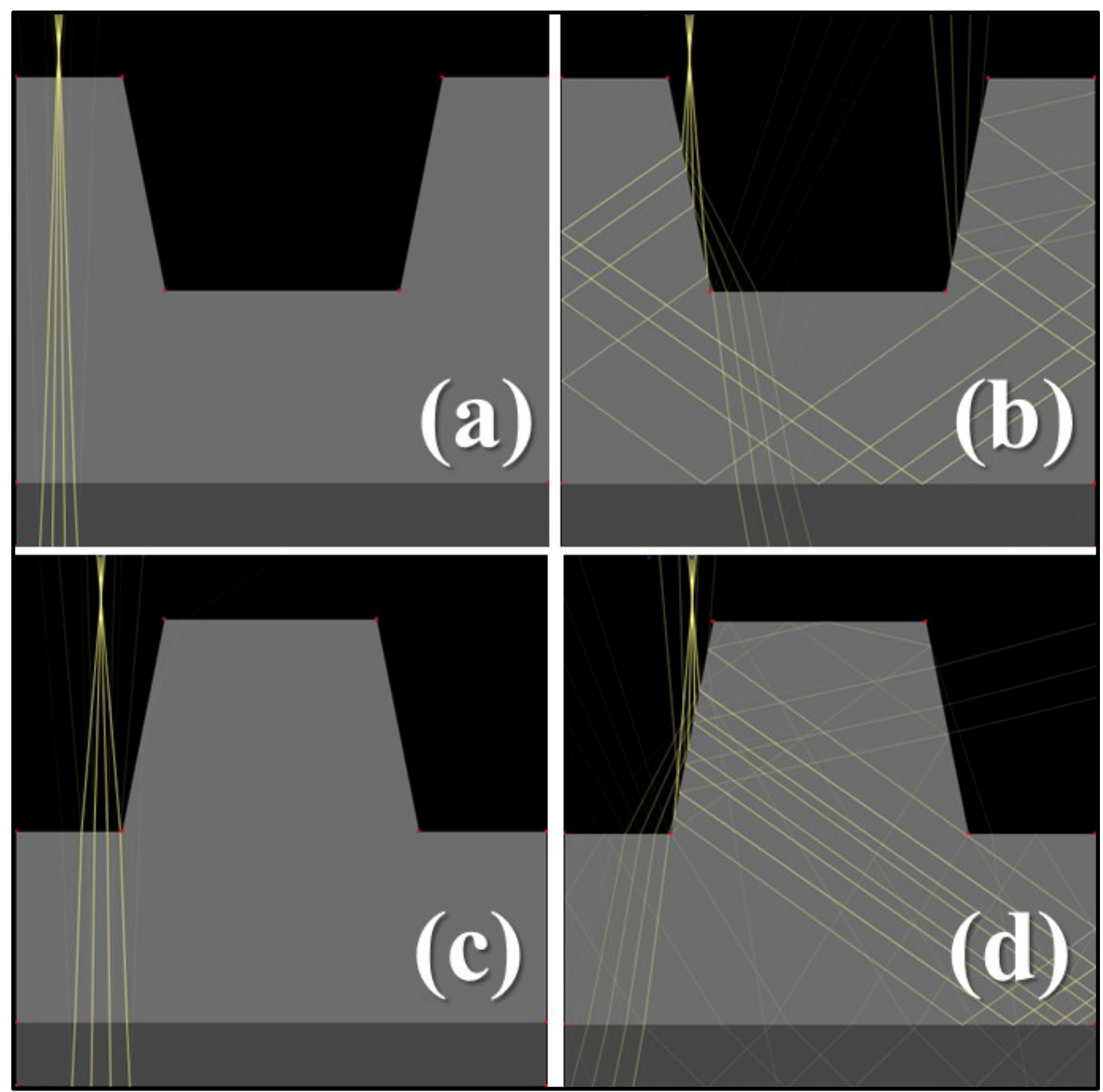

Figure 4.42: Simulation of GaN pits.

defect density, light will have a longer beam path within the sample. Higher defect densities will increase absorbance where the light hits the sample, but also will increase scattering so that less of the light propagates through the sample. The waveguiding 
hypothesis shows this result: The lower defect density samples have better signal-tonoise ratios and better resolution (as shown in Figure 4.32). Similarly, a dirty sample surface could scatter light that is refracting within the sample. Clean samples yield higher and better CPC signals.

We can also return to look at the taller (i.e. longer etch time) pillars in Figure 4.33. With the taller pillars, there is a greater surface area for the light to be incident upon, increasing the opportunities for the light to have extended beam paths. The waveguiding hypothesis also matches well with this result. We see higher signals with taller pillars.

The waveguiding hypothesis can also explain why the piezoelectric effects did not affect the CPC signal, see Figure 4.28. We also see that the induced electric field does not increase the signal either, see Figure 4.30. Therefore, only modifying the parameter of the beam paths would improve the CPC signal, and not the material's electric-field properties.

This result is important for the development of the CPC-STED system. The STED system depends on the absorption of photons, not a secondary effect like we see in the scanning-CPC results of the GaN samples. If we apply the STED technique to the scanning-CPC technique, we cannot expect the same resolution improvements, because with the depletion and excitation beam having two different energies they will refract slightly differently in the GaN. The path of the depletion beam will diverge from the path of the excitation beam, therefore we cannot expect a $100 \%$ efficiency of the CPC-STED process, as we will explore in the next chapter. 


\section{CHAPTER 5 CPC-STED SCANNING RESULTS}

With the success of the scanning-CPC results, we sought to apply the principles found in Stimulated Emission Depletion (STED) fluorescence microscopy to develop Capacitive Photocurrent Stimulated Emission Depletion (CPC-STED) microscopy. This chapter will discuss the theoretical framework for CPC-STED and then discuss the results of using this technique. Then various experimental setups and material systems will be discussed as well as the issues that arose and how they were resolved.

\subsection{Theoretical framework}

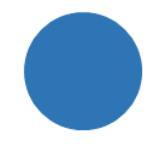

(a) $2.54 \mathrm{eV}$ Excitation Beam (Gaussian)

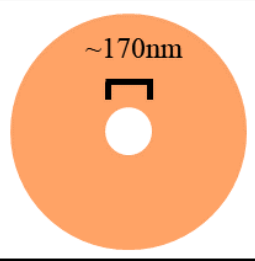

(b) $2.09 \mathrm{eV}$ Depletion Beam (Toroidal)

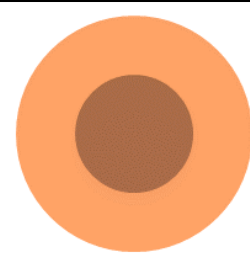

(c) 2.54 / $2.09 \mathrm{eV}$ Bleaching Overlap

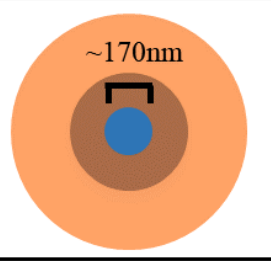

(d) 2.54 / $2.09 \mathrm{eV}$ STED Overlapping

Figure 5.1: STED microscopy beam layout. (a) excitation beam only, (b) toroidal depletion beam, (c) overlapping of gaussian depletion and gaussian excitation beam, and (d) collinear toroidal depletion beam and gaussian excitation beam.

Since fixed point CPC and scanning-CPC both detect the AC signal generated from a sample by either a pulsed or chopped light source, this process could allow for the utilization of the techniques developed in fluorescence STED microscopy. In Figure 5.1, we see how STED achieves increased resolution improvements: (a) shows the excitation only beam, (b) shows the toroidal depletion beam, (c) shows the excitation beam overlaid with a Gaussian depletion beam, and (c) shows the toroidal depletion overlaid with the 
excitation beam, the configuration used for CPC-STED. The resolution improvement is done by reducing the effective spot size below an objective's diffraction limit through the depletion of a samples signal in the portions of the excitation beam that is overlapped by the sufficiently intense portions of the toroidal depletion beam, as we will discuss.

In our case, the diffraction limit of the $2.54 \mathrm{eV}$ beam when using an objective with numerical aperture of 0.8 is given by:

$$
d=\frac{\lambda}{2 * N A}=\frac{488 \mathrm{~nm}}{2 * 0.8}=305 \mathrm{~nm}
$$

with the toroid having an effective center hole as small as $\sim 170 \mathrm{~nm}$ when using the 0.80 numerical aperture (NA), $3.5 \mathrm{~mm}$ working distance, 100x objective. This spot size can be reduced to $\sim 20 \mathrm{~nm}$ when using a higher NA objective with a narrower working distance, like the oil-emersion lens used for STED fluorescence microscopy. Since we are interested in studying surface properties, we can only use the $100 \mathrm{x} / 0.80$ objective.

We also need to look at how effective the toroid is and what effective spot size we can get from the system. Based on the work in fluorescent STED microscopy, we have a method for determining the theoretical limits to the effective spot size.

$$
d \approx \frac{\lambda}{2 N A\left(1+I / I_{S}\right)^{1 / 2}}
$$

where $d$ is the effective spot diameter, $\lambda$ is the wavelength of the depletion beam, $N A$ is the numerical aperture of the objective being used, $I$ is the intensity of the depletion beam, and $I_{s}$ is the threshold intensity needed to deplete the sample; this value is a material dependent property [38]. 
In Figure 5.2, we see how the material dependent feature, $I_{s}$, affects the effective spot size. As the depletion beam's intensity increases, the red (1x), blue (2x), and green (4x) traces, the effective spot size moves in toward the center of the gaussian excitation beam. That means with a sufficiently low $I_{S}$ or a high depletion intensity the ratio of the two, R, can be increased to create a nearly infinitely small theoretical effective spot size.

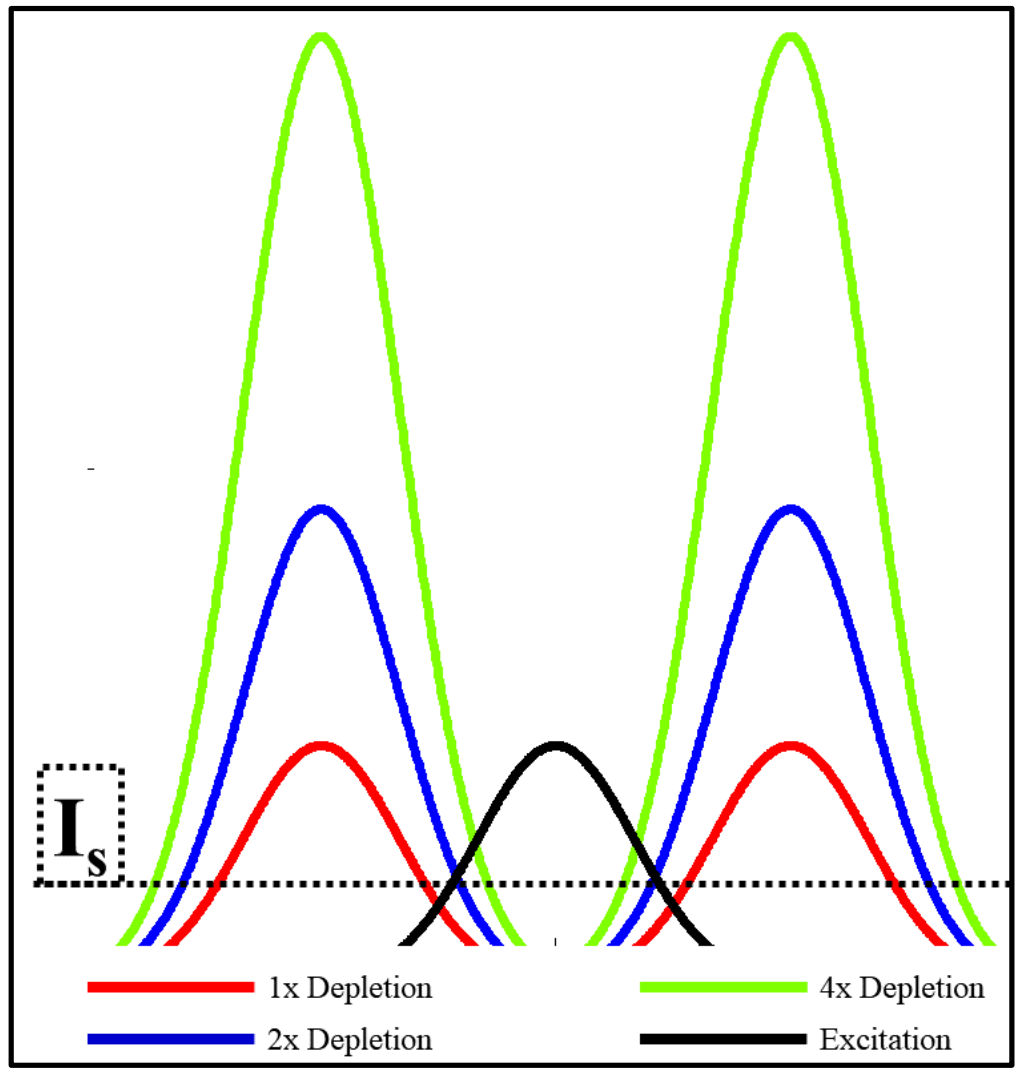

Figure 5.2: Diagram showing the importance of $\mathrm{I}_{\mathrm{s}}$ and depletion intensity.

If we assume that a sample has a similar minimum response intensity value, $I_{s}$, such that it is the lowest intensity required to prevent a material system from generating an $\mathrm{AC}$ signal from the modulated excitation beam, then we can define a value,

$$
R=I / I_{s}
$$

to determine the appropriate parameters needed to overcome the diffraction limit of a $2.54 \mathrm{eV}$ based system, at $305 \mathrm{~nm}$. From Figure 5.3, we can see that we need a value of 
$R \geq 0.47$ to have an effective spot size smaller than $305 \mathrm{~nm}$. Since the $2.09 \mathrm{eV}$ laser has a nominal range of $150 \mathrm{~mW}$ to $1650 \mathrm{~mW}$, the maximum value we have available is

$$
R=I / I_{s}=1650 / 150=11
$$

giving a theoretical minimum effective spot size of $d \approx 107 \mathrm{~nm}$, since the depletion beam can vary form $150 \mathrm{~mW}$ to $1650 \mathrm{~mW}$ we can look at the model to determine the best case scenario there the material has a $I_{s}$ at the minimum power of the depletion beam.

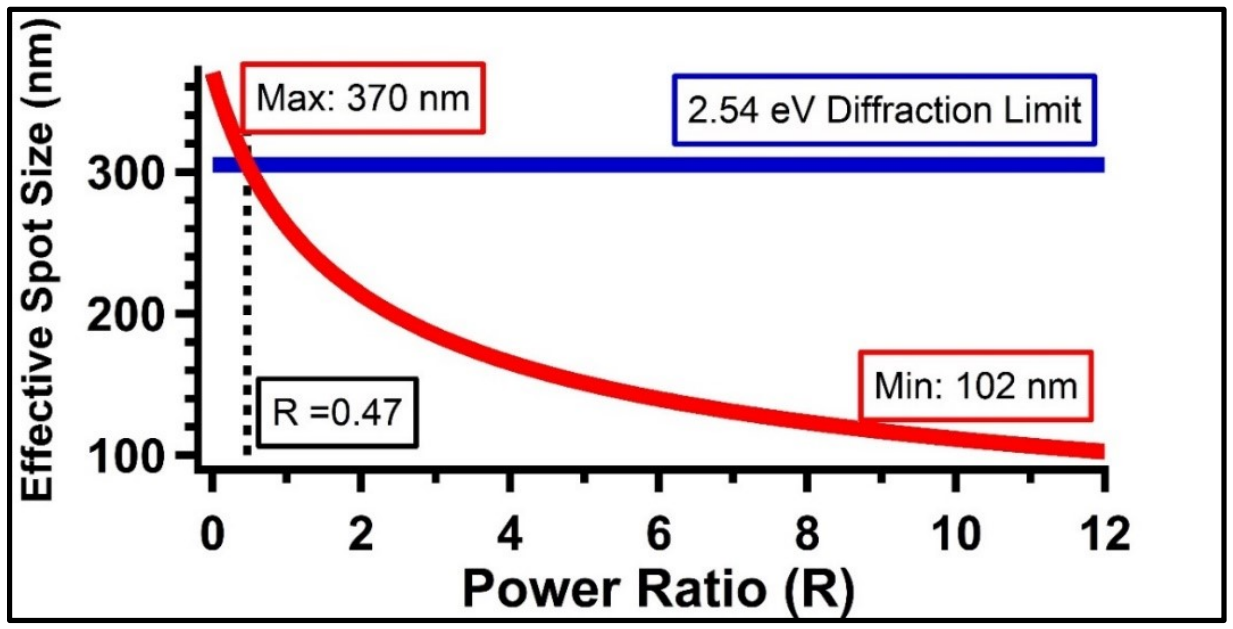

Figure 5.3: Theoretical limits of CPC-STED effective spot size, using a $N A=0.80$ objective.

5.2 Preliminary work

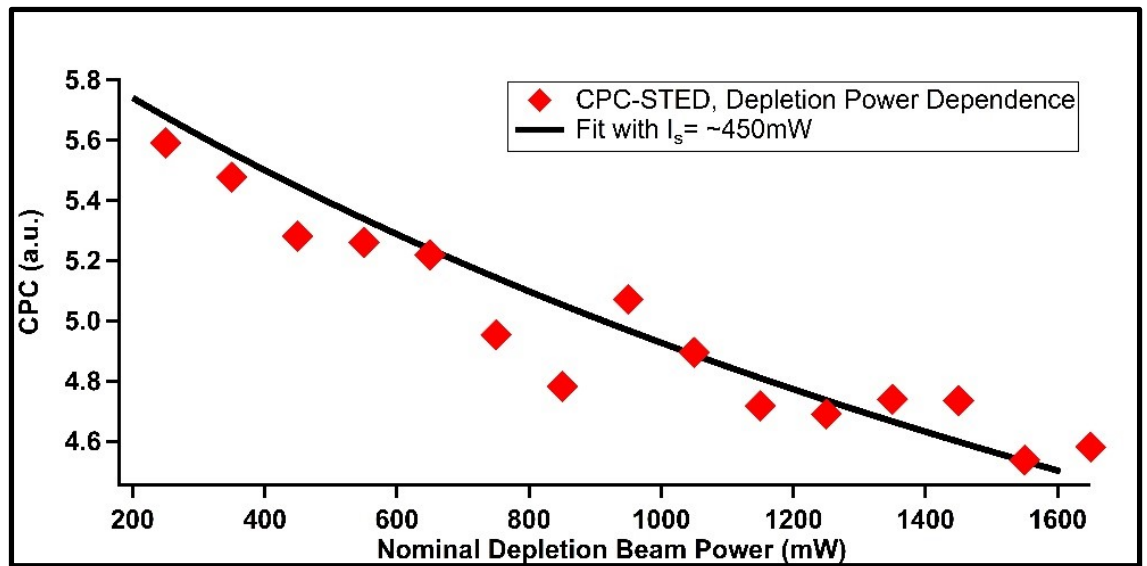

Figure 5.4: Fit of depletion intensity with CPC signal.

Since we now know how the minimum depletion intensity threshold controls the effective spot size, we sought to estimate this value. To determine the value, we 
performed scans of $\mathrm{GaN}$, maintaining a constant excitation beam power, and varying the depletion beam power from $150 \mathrm{~mW}$ to $1650 \mathrm{~mW}$, the limits of the $2.09 \mathrm{eV}$ laser. The result is the plot seen in Figure 5.4, where the CPC signal decreases as the depletion beam power increases, because as the depletion beam power increases, the effective spot size shrinks. There is also a fit of Equation (5.2) with $\mathrm{I}_{\mathrm{s}}=450 \mathrm{~mW}$. With this $\mathrm{I}_{\mathrm{s}}$ value and using the laser's maximum power of $1650 \mathrm{~mW}$, we have a value of $\mathrm{R}=3.67$ meaning that the minimum theoretical spot size we can expect is $d \approx 171 \mathrm{~nm}$, which is much smaller than the diffraction limit of our $2.54 \mathrm{eV}$ beam at $\mathrm{d}=305 \mathrm{~nm}$. Therefore, if we can resolve details in the scans that are smaller than $305 \mathrm{~nm}$, then subdiffraction imaging is achieved.

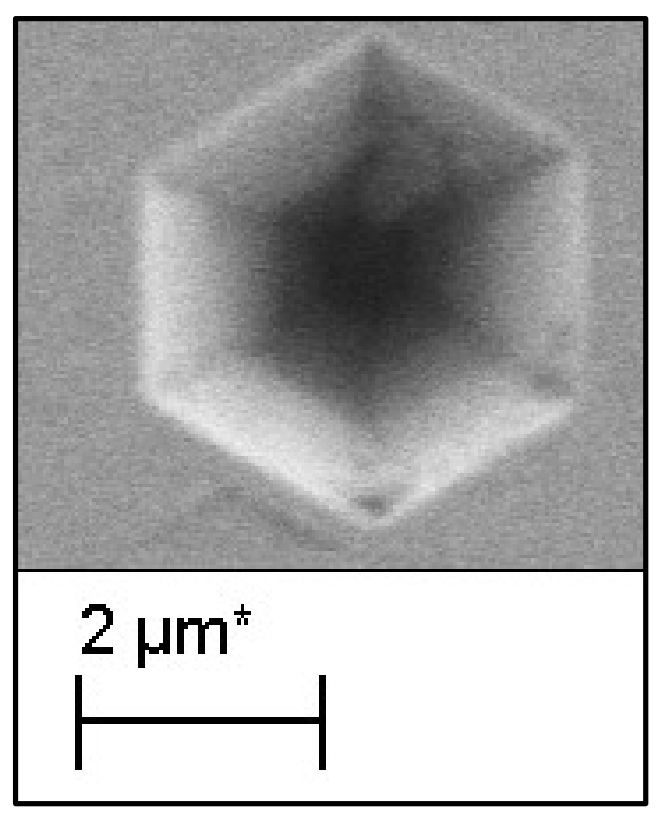

Figure 5.5: SEM of GaN pit.

The depletion beam is employed to stop the signal in its region, so we can test the effectiveness of this beam by overlapping a Gaussian depletion beam and a Gaussian excitation beam, as seen in Figure 5.1 (c). We refer to this as a bleaching test since the higher power, $\mathrm{CW} 2.09 \mathrm{eV}$ beam should 'bleach out' the signal generated by the chopped 


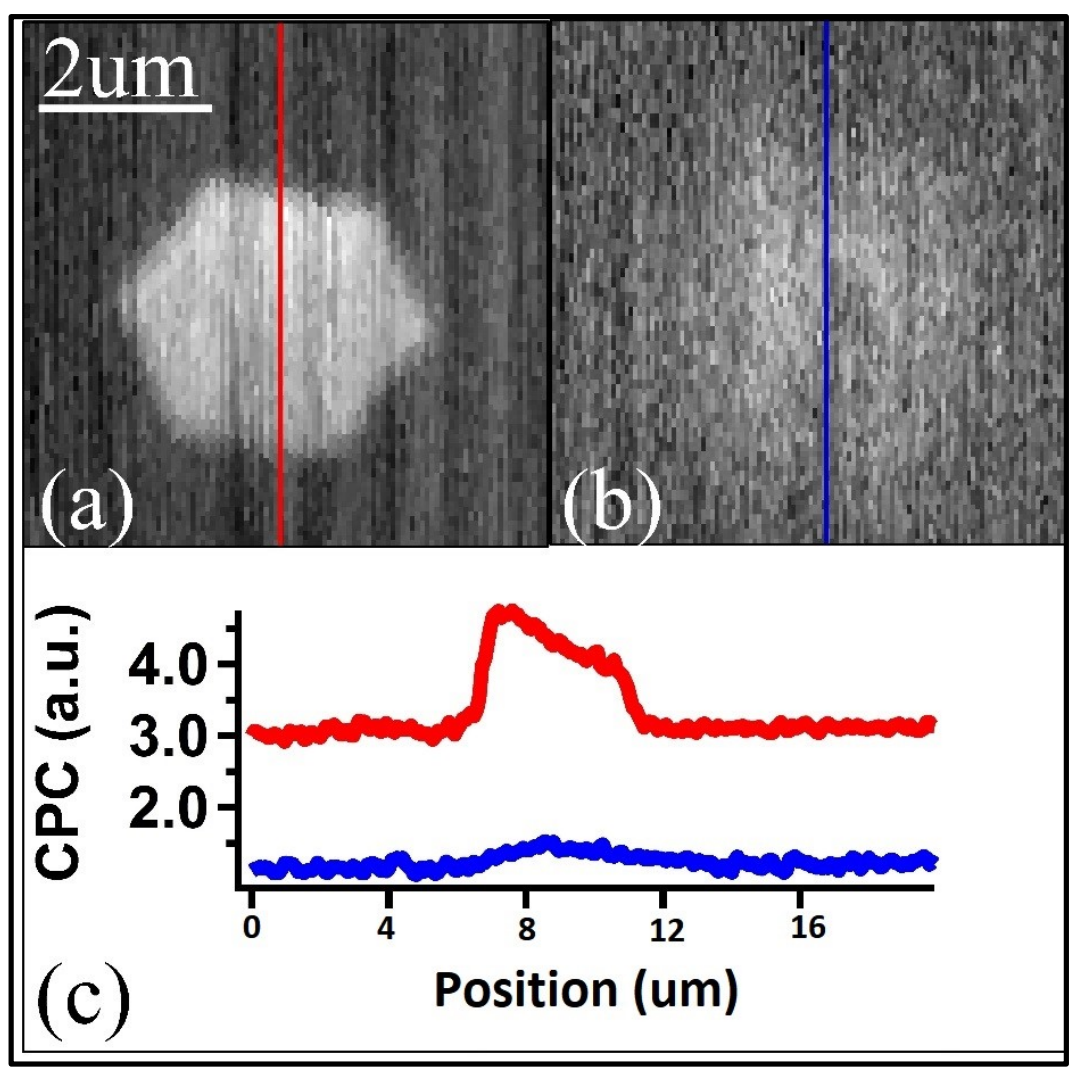

Figure 5.6: Bleach test of GaN pits. (a) bleached image, (b) excitation beam only image, (c) CPC-STED image, and (d) line scans form each image.

$2.54 \mathrm{eV}$ beam. In Figure 5.6, we have the results of this bleaching test done on a GaN pit like the pit seen in Figure 5.5, showing a few features of importance, including that the bleached image (b) has a significantly decreased signal both in the pit area and in the bulk of the unetched GaN. The resolution is also reduced in the bleached (b) image when compared to the image generated from using only the $2.54 \mathrm{eV}$ excitation beam (a). This result is as expected, since the bleached image should have the lowest signal at every point since every point of the excitation beam is bleached.

We need to see how the contact configuration modifies the CPC signal we get and how this in turn determines the effectiveness of the CPC-STED. As discussed earlier, we used a contact to go around the scan area, providing a more symmetrical image. In Figure 
5.7, we see a set of comparisons of excitation only vs CPC-STED in both inside a circular contact and outside of a circular contact, as well as line scans from these layouts. We see that in the shallow GaN pits there is limited asymmetry initially and that being inside the contact does remedy the asymmetry slightly. We also see that the signal strength is

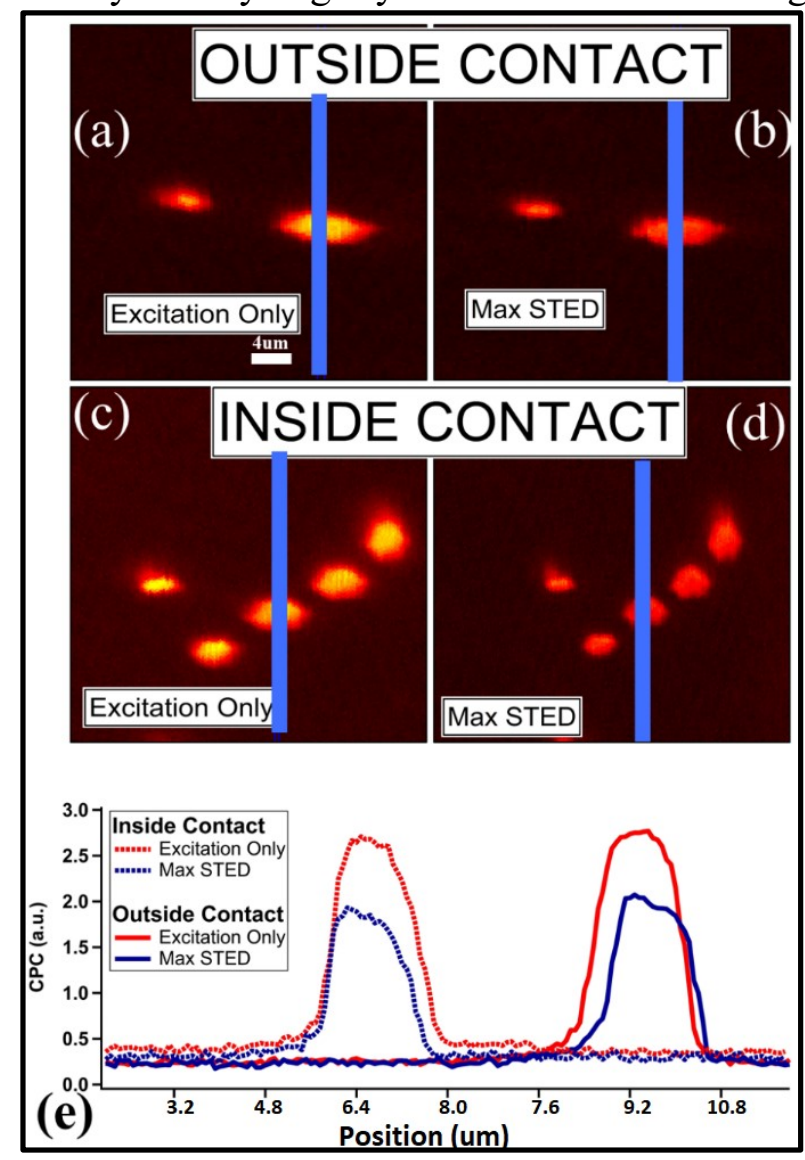

Figure 5.7: Comparing contact location with CPC-STED functionality. Outside the contact with (a) excitation only and (b) CPC-STED, inside the contact with (c) excitation only and (d) CPC-STED, and (e) line scan comparisons.

independent of contact style. There is slight resolution improvement or narrowing of the signal given from the pits when using the CPC-STED configuration.

With the theoretical framework and results of the bleaching test, we were ready to capture images using the CPC-STED system. We know that to prove super resolution, we must resolve features smaller than $305 \mathrm{~nm}$. We should be able to resolve features as small 
as $171 \mathrm{~nm}$. We also know that the principles of the system are valid, as seen in the bleaching results.

\subsection{Early results}

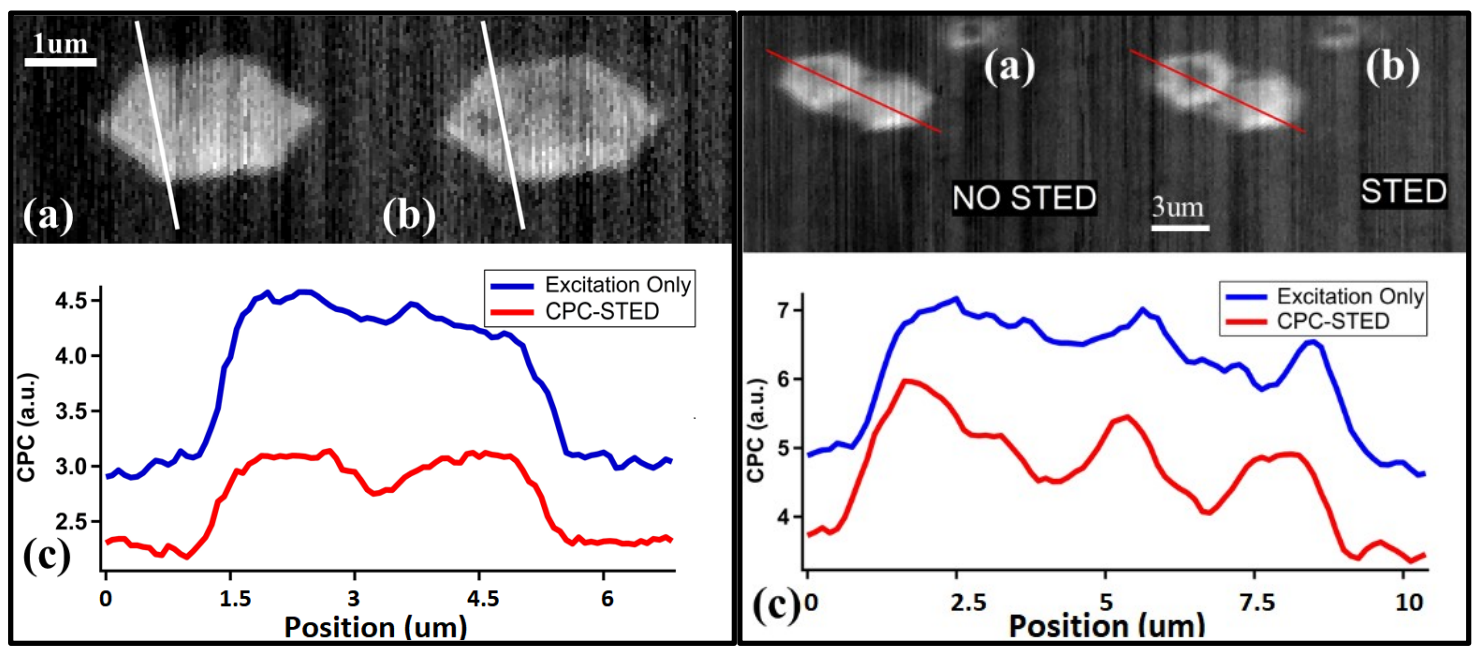

Figure 5.8: GaN pits with excitation only Figure 5.9: More GaN pits with excitation (a) and CPC-STED (b) with a line scan (c) only (a) and CPC-STED (b) with a line across both. scan (c) across both.

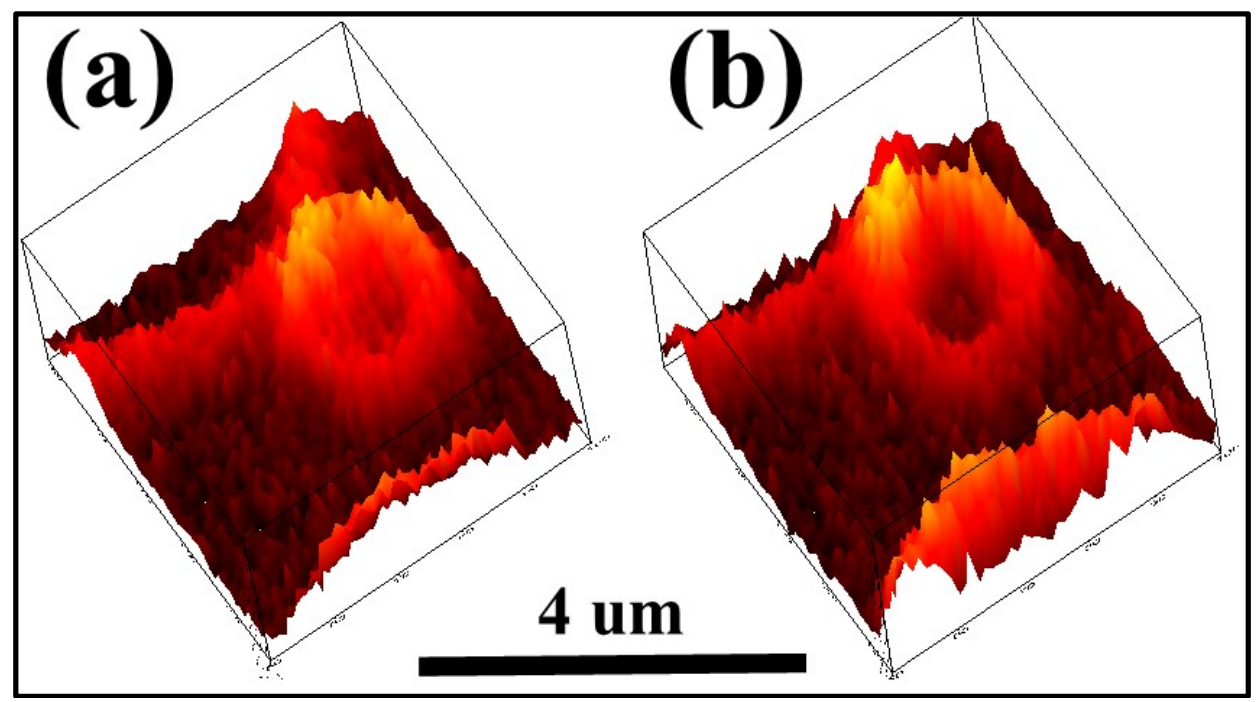

Figure 5.10: (a) Minimum depletion power (b) Maximum depletion power image of pillars in GaN

Returning to the familiar system of GaN pits, we could capture the images seen in

Figure 5.8 and Figure 5.9. In both, we see the results of collecting an image using only the $2.54 \mathrm{eV}$ excitation beam (a), and the results of using the CPC-STED system (b), as 
well as the line scans across these features. The next step is to optimize the system utilizing the values we determined for $\mathrm{I}_{\mathrm{s}}$.

We can look at the familiar GaN pillars, as seen in the 3-dimentional plotting in Figure 5.10. In the figure (a) is using a $R=1$ value (depletion power at $450 \mathrm{mw}$ ) and (b) is using a $\mathrm{R}=3.67$ value (depletion power at $1650 \mathrm{~mW}$ ). Since we are scanning a robust semiconductor we do not need to be overly concerned with power considerations, allowing for utilizing the full power range of the light sources. Applying line scans across the features in Figure 5.10 show an improved peak to valley ratio, as shown in the lines scans in Figure 5.11 where the blue trace is using a lower depletion power than red trace, which uses the highest available depletion power. With a higher-powered depletion beam, the outer parameter of the excitation beam has an improved resolution since the signal is further ensured to come only from a smaller effective spot size. Combining the improvements seen with higher power depletion beams and different energies for the depletion and excitation beam, we approach the best resolution improvements possible in the current system. This improvement also requires employing a round contact to minimize the asymmetry of the waveguiding effect, ensuring that all electron-hole separations are relatively equidistant to a contact, rather than one sided.

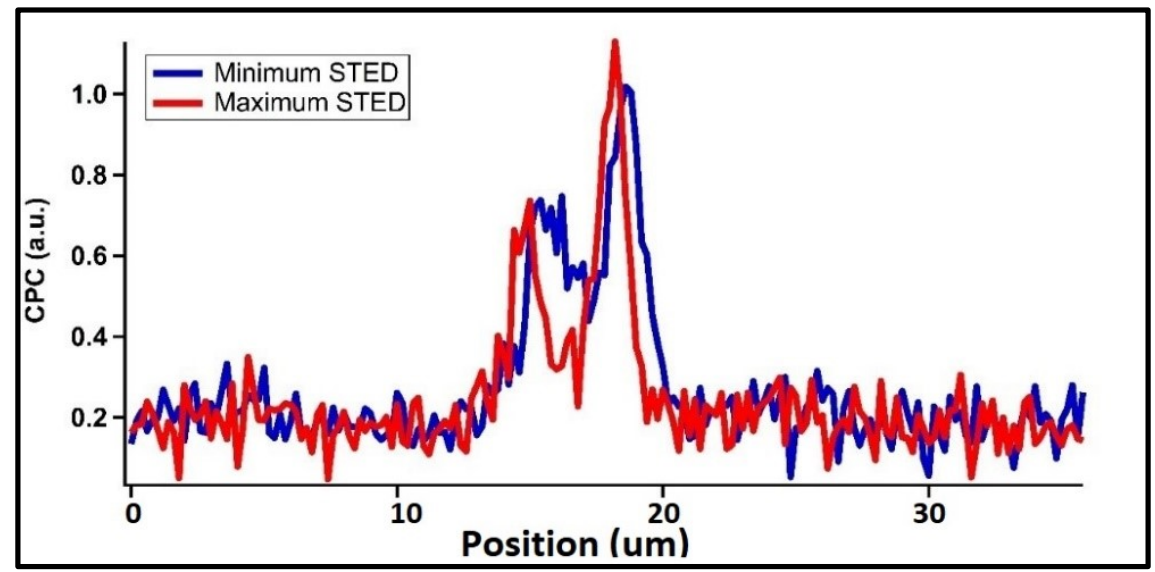

Figure 5.11: Lines Scans of the GaN pillar features in Figure 5.10 
A final variable we can control is the power of the excitation beam. In Figure

5.12, we can return to the previously used GaN pits to see how strong of an improvement we can have using higher excitation beam powers. We see that the excitation power is not as important as the increase in the depletion power. We can apply these constraints when evaluating excitation only line scans and CPC-STED line scans.

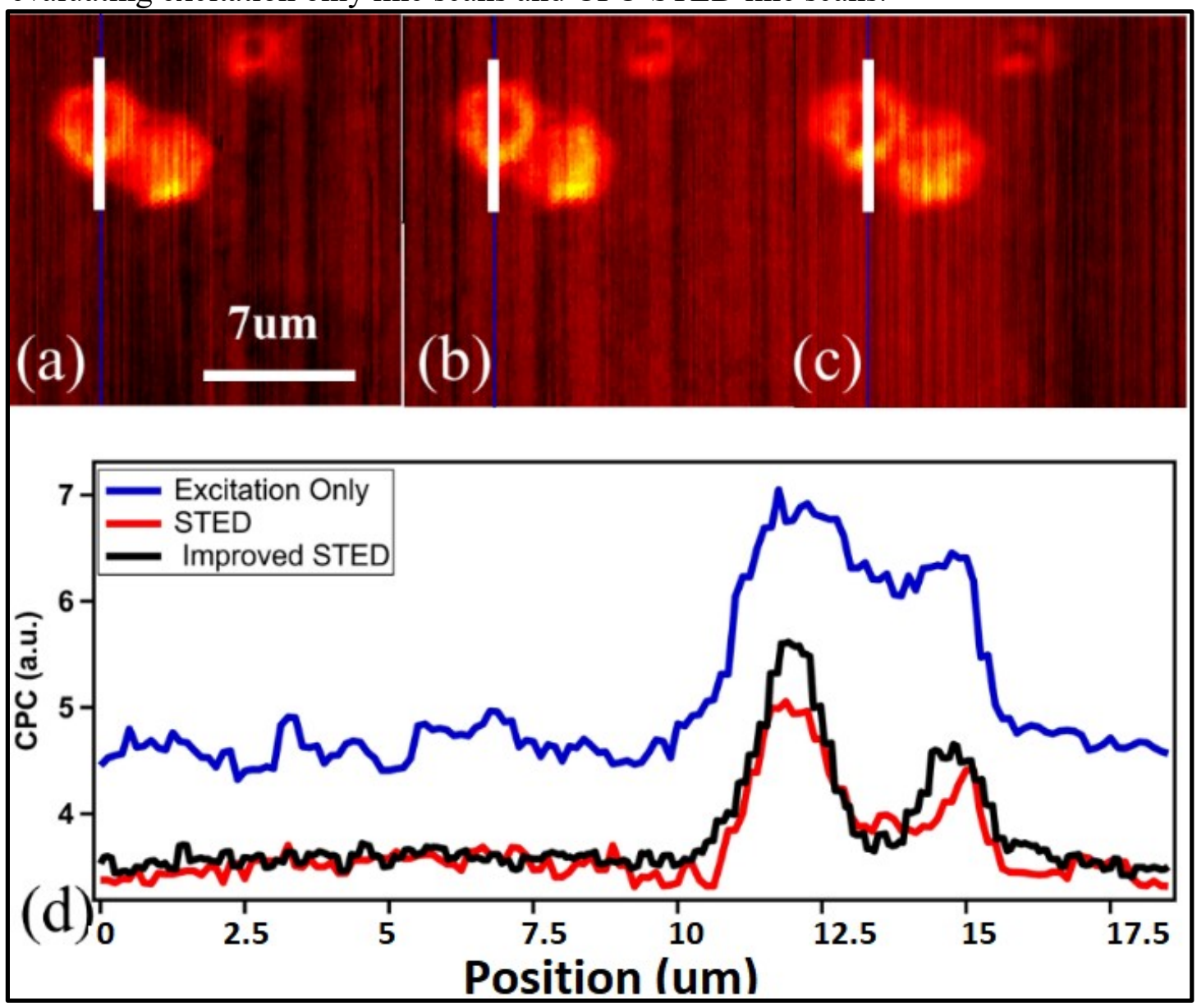

Figure 5.12: A comparison of (a) excitation only, (b) low excitation power CPCSTED, (c) high power excitation CPC-STED, and (d) line scans of each image. 


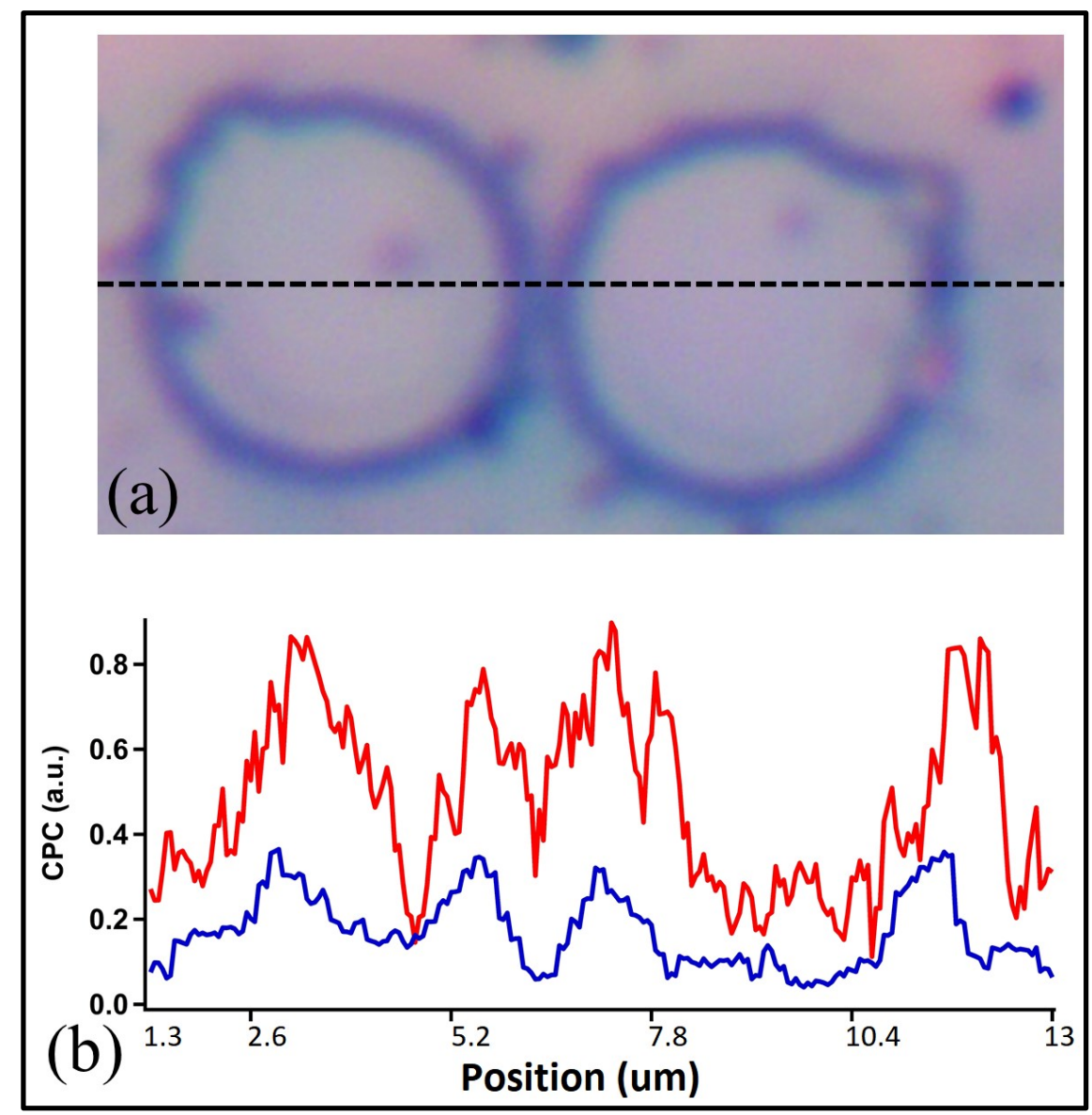

Figure 5.13: (a) Photo of pillars fabricated in a GaN wafer, with a dotted line along the line scan path and (b) line scans for excitation only in red and CPC-STED in blue.

Now, we can design the experiment such that the best $\mathrm{R}$ value is used, $\mathrm{R}=3.67$, the highest excitation beam power is used, and a round contact is employed. Looking at Figure 5.13 (a), we see pillars in GaN that are drawing nearer and doing a line scan of the close pillars (b) we see familiar results. The CPC-STED signal is below that of the excitation only, but the excitation beam only signal has a signal to noise ratio of 2.60 and the CPC-STED has a signal to noise ratio of 5.73. Also, we can see that the CPC-STED curve (blue) resolves at the pillar separation point where the excitation only line scan does not. In Figure 5.13 (b), based on the measurements of the separation of the peaks the excitation only line scan cannot resolve below $\sim 400 \mathrm{~nm}$ at approximately point 175 , 
where we can see the CPC-STED resolves down to $\sim 240 \mathrm{~nm}$. When using an $\mathrm{R}=3$ ratio, which we get from optimizing the fit in Figure 5.4 and Equation (5.2) we find an effective spot size of $\sim 171 \mathrm{~nm}$, significantly below the diffraction limit of the $2.54 \mathrm{eV}$ beam, $305 \mathrm{~nm}$. Therefore, we see that we are resolving below the diffraction limit, yet there is still room for improvement. We see that the system has some inherent

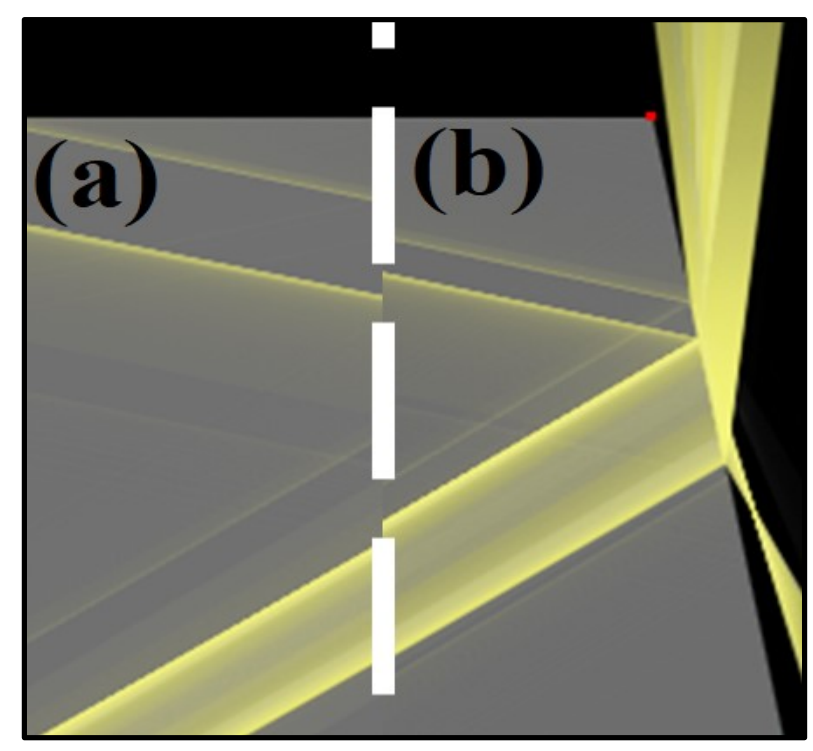

Figure 5.14: RayOptics simulation of the (a) $2.54 \mathrm{eV}$ excitation and (b) $2.09 \mathrm{eV}$ depletion beam path through the wall of a GaN pit.

inefficiencies, where the ratio of effective spot size to resolved spot size are very similar. For excitation only we see a theoretical spot size $\left(\mathrm{S}_{\mathrm{T}}\right)$ and an experimental spot size $\left(\mathrm{S}_{\mathrm{E}}\right)$, $\frac{S_{T}}{S_{E}}=\frac{305 \mathrm{~nm}}{400 \mathrm{~nm}}=0.7625 ;$ likewise, for CPC-STED we have, $\frac{S_{T}}{S_{E}}=\frac{171 \mathrm{~nm}}{240 \mathrm{~nm}}=0.7125$.

With the STED-CPC signal showing a waveguiding effect, as discussed in Section 4.4, this waveguiding effect directly influences the resolution improvement due to $\mathrm{GaN}$ having a wavelength dependent index of refraction meaning that in the regions of overlapping excitation and depletion beams will not follow the same paths. Looking at Figure 5.14, we see that the beam path of the $2.54 \mathrm{eV}$ excitation beam (a) is not completely overlapped by the $2.09 \mathrm{eV}$ depletion beam (b). While there will be 
overlapping for a majority of the area, the interior of the toroid has reduced effectiveness, while increasing the theoretical minimum spot size. This change is negligible in CPCSTED because the depletion beam is utilized for its $\mathrm{CW}$ properties rather than its energy dependent effects on a sample, as it is used in fluorescence STED.

\subsection{Adjusting design parameters}

This section will discuss the various parameters we adjusted to further improve the system. First, we considered investigating the excitation and depletion overlapping by employing identical energy excitation and depletion beams. Next, we look at a nonsemiconductor system. Finally, we look some of the details of the experimental design.

\subsubsection{Dual 2.09eV setup}

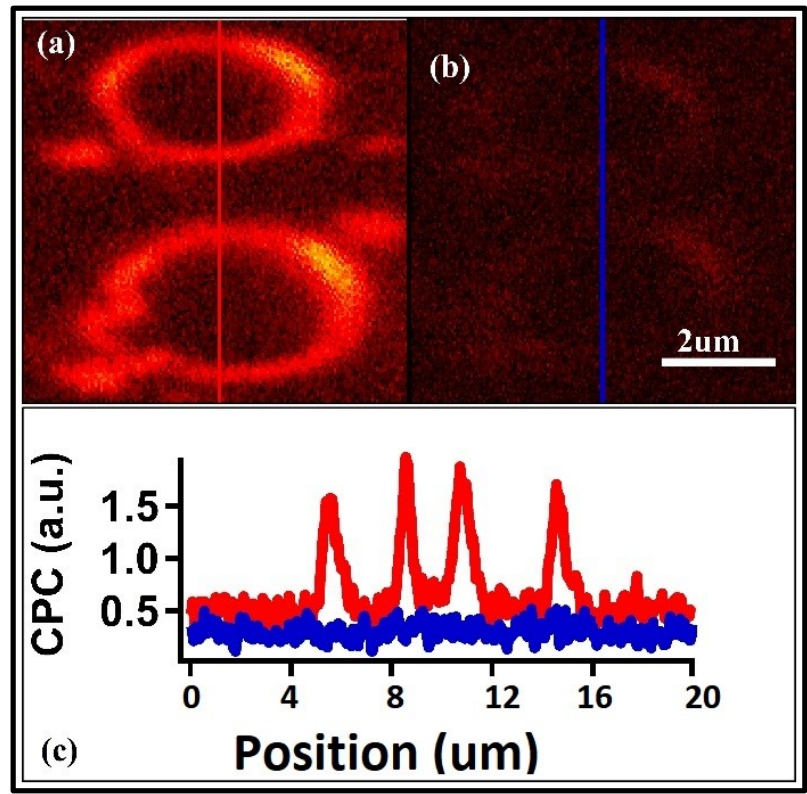

Figure 5.15: Bleach test of dual $2.09 \mathrm{eV}$ beams with (a) $2.09 \mathrm{eV}$ excitation only (b) dual bleaching $2.09 \mathrm{eV}$, and (c) line scans through the center of the images

With the concerns of the wavelength dependent index of refraction, we modified the system to have $2.09 \mathrm{eV}$ as both the excitation and depletion beam since our system is 
possibly not as energy dependent as standard STED fluorophores. We adjusted the location of the chopper and the steering of the beam as described in Section Optical table layout and Figure 3.3. The first step is to do a bleach test. We can see in Figure 5.15 (a) that using the $2.09 \mathrm{eV}$ beam as the chopped excitation beam provides a suitable signal, but the resolution is not impeded as much as in a $2.54 \mathrm{eV} / 2.09 \mathrm{eV}$ setup.

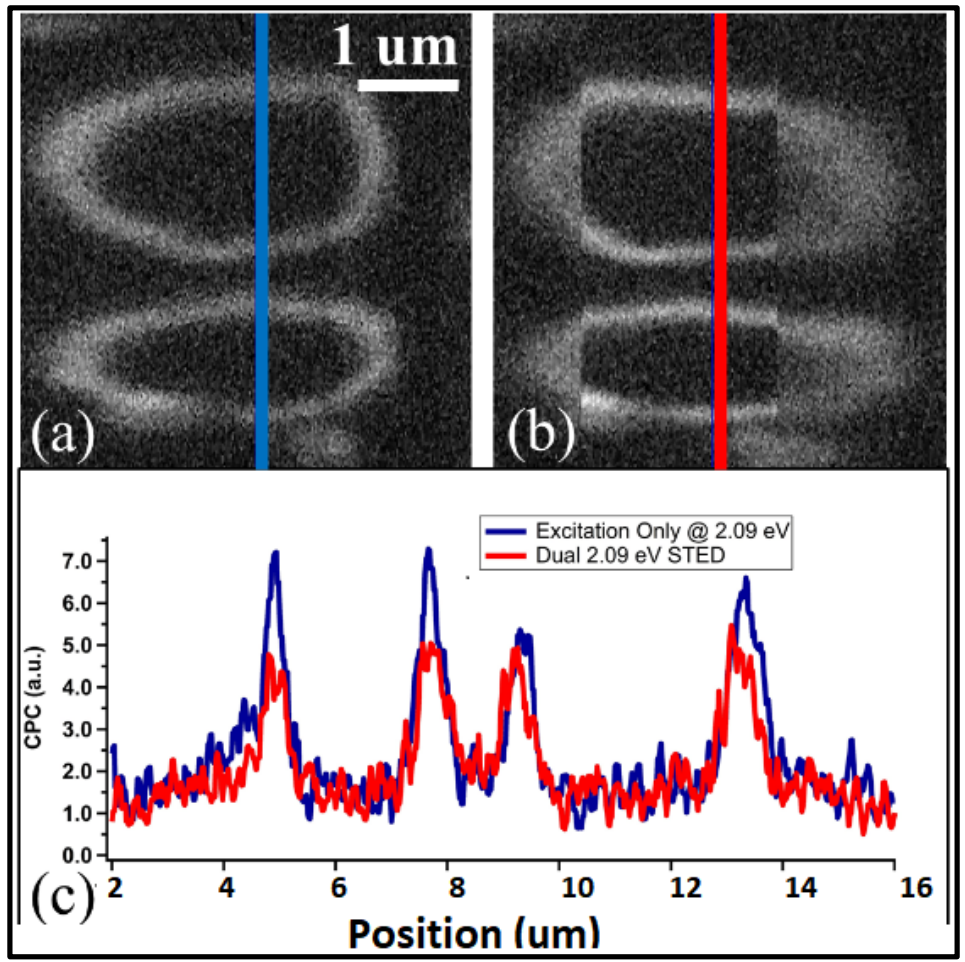

Figure 5.16: (a) $2.09 \mathrm{eV}$ excitation only, (b) dual $2.09 \mathrm{eV}$ CPC-STED, and (c) line scans across the GaN pillars

The $2.09 \mathrm{eV}$ beam was also used for this because it has a significantly larger power range for the excitation beam, maxing out at $\sim 400 \mathrm{~mW}$ compared to the maximum power of the $2.54 \mathrm{eV}$ at $60 \mathrm{~mW}$. Although, since we are splitting the beam the maximum depletion power is $\sim 400 \mathrm{~mW}$ meaning that the best value we can get is $\mathrm{R} \approx 1$ for the theoretical minimum spot size, which limits the effectiveness of this setup, meaning that our theoretical minimum spot size is $\mathrm{d} \approx 262 \mathrm{~nm}$, and is significantly higher than the theoretical minimum spot size for the primary setup at $\mathrm{d} \approx 171 \mathrm{~nm}$. We can see the effects 
of this in Figure 5.16, where using different excitation and depletion energies shows improvements in peak to valley ratios, using identical energies for depletion and excitation show a decrease in the peak to valley ratio. There also is no resolution improvement as seen from the lack of narrowing in the peaks or increase in separation between parts of the peaks. This lack of improvement is likely due to not having an insufficient depletion beam power, nominally at $\sim 400 \mathrm{~mW}$. Therefore, the dual $2.09 \mathrm{eV}$ setup is not adequate for our test. With this setup being unsuccessful we tried a previous material system.

\subsubsection{Perovskite}

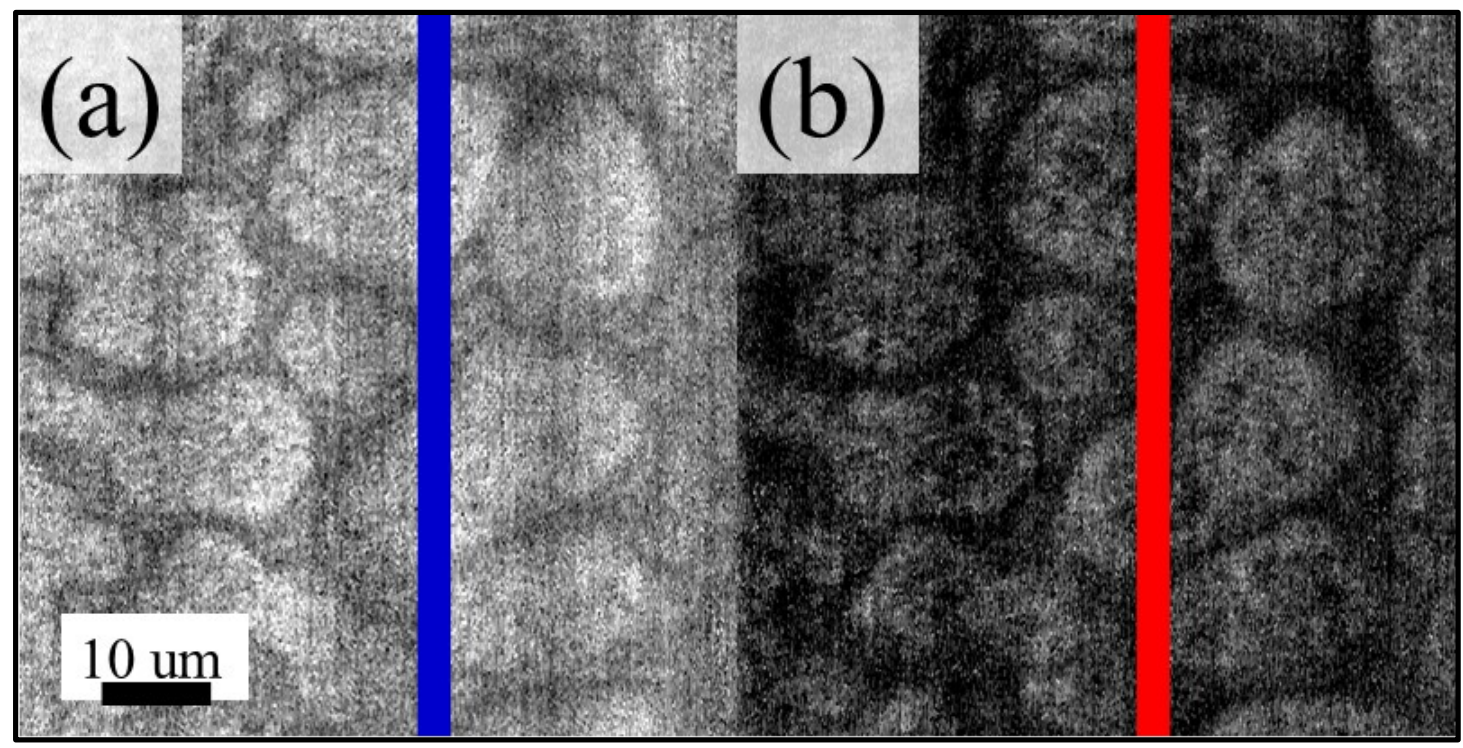

Figure 5.17: (a) $2.54 \mathrm{eV}$ excitation only and (b) CPC-STED of perovskites.

Since the dual $2.09 \mathrm{eV}$ beams did not result in an improved imaged, we studied an alternative material system. Since the perovskite responded to lower power, we returned to that system anticipating that using a very lower power would not burn the sample, and the addition of a depletion beam would increase the effectiveness of the excitation beam by improving the signal to noise ratio. In Figure 5.17, we see the image results of the perovskite system. With a lower power in the excitation only image (a), we have a less 


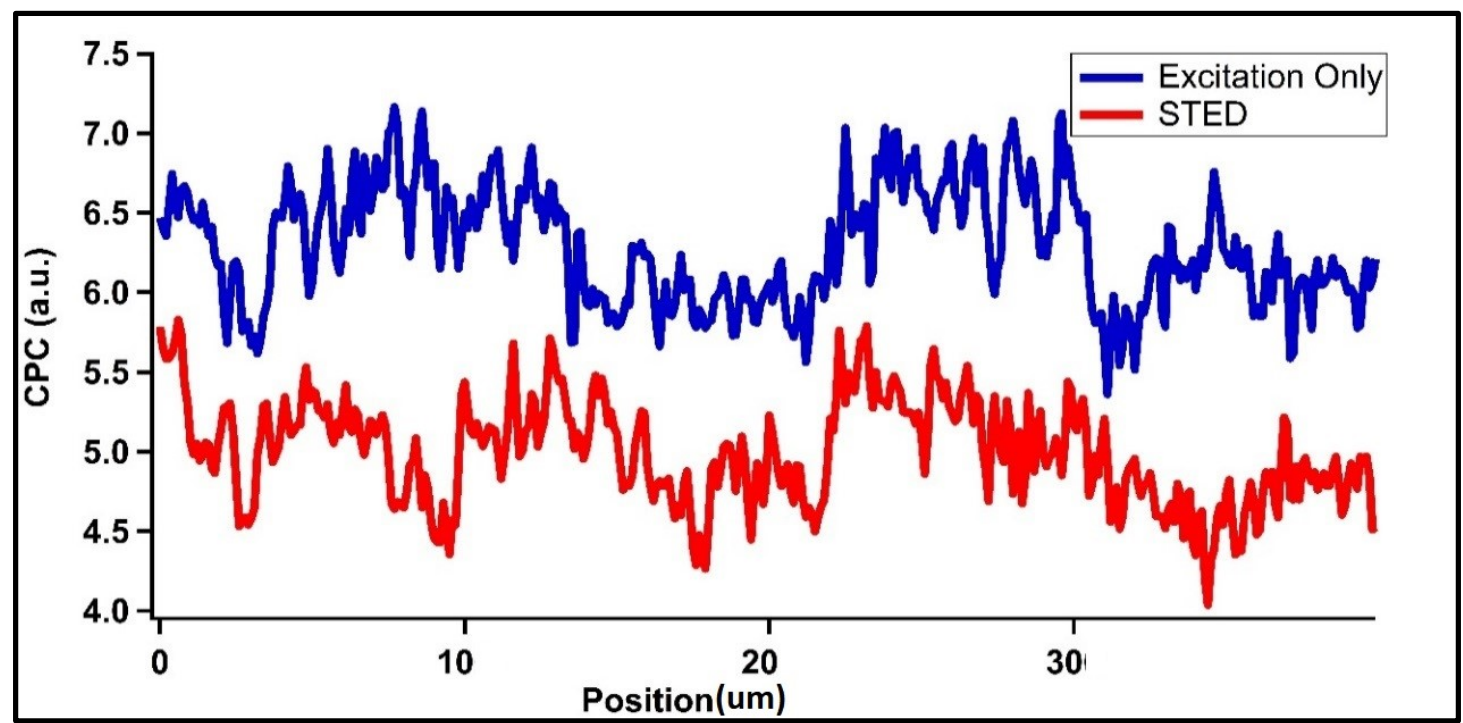

Figure 5.18 :Line scan across the images in Figure 5.17

defined image than we have seen. The addition of the toroidal depletion beam does seem to improve the image quality (b). We can see from the line scans in Figure 5.18 of both images that the overall decrease in signal when using full CPC-STED functionality carries through to the perovskite system, as seen in the GaN system. We also see some improvements in the signal to noise ratio as seen at point 95, but this improvement does not lead to a detectable increase in resolution. The problem with the perovskite system is the overall signal is still very low making it difficult to show improvements. The perovskites were also deteriorating quickly in the typical open-air configuration of the system and holder means it was not suitable to extensive study.

\subsection{Obstacles and resolutions}

We ran into a significant problem through the course of this work. Around mid2016, the MadCityLabs stage with angstrom resolution, had a critical failure in its piezoelectric actuators, which was likely due to its age. This issue forced us to use the less optimal Prior Scientific stage, with its 40nm minimum step size. This new stage required a complete rewriting of the LabView software that controls the stage, collects 
the data, and stores the data. This software development is detailed in Chapter 3.3.

Overall, the new stage and new software sacrifices some resolution for greater control

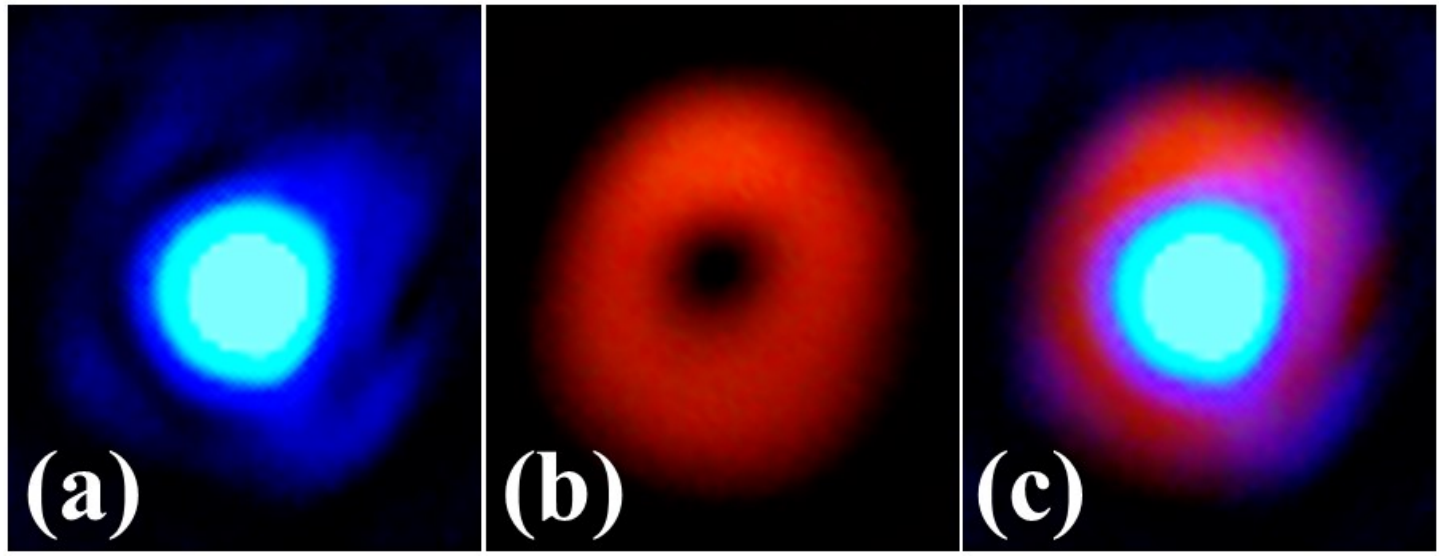

Figure 5.19: Photographs from the camera on the microscope showing proper alignment.

over the scan area and the minute details of the stages motion, such as speed, acceleration, scan area size. Previously, we were limited to maximum scan areas of $\sim 45$ um x $45 \mathrm{um}$ now we have a limit of $\sim 10 \mathrm{~cm}$ x $10 \mathrm{~cm}$. Since we have a minimum theoretical spot size for CPC-STED of $\sim 170 \mathrm{~nm}$, the $40 \mathrm{~nm}$ step size is not an issue, yet it would be for fluoresce STED with its spot sizes of $<5 \mathrm{~nm}$.

While solving the issues involved with installing a new stage, we encountered additional issues that facilitated implementing permanent improvements. An initial issue was getting a high-quality alignment of the toroidal depletion beam and the Gaussian excitation beam. Another issue was the mechanical z-axis drift of the stage due to the microscope's stage, but not the scanning stage. To solve both of these issues, we added a camera [MU1000-CK 10MP, AmScope] to the system via the top mount on the microscope. This camera came with proprietary software that allows for live viewing of the sample surface and can assist directly in completing the CPC-STED alignment. In Figure 5.19, we have photographs taken with this camera that shows just the $2.54 \mathrm{eV}$ 
excitation beam (a), the $2.09 \mathrm{eV}$ toroidal beam (b), and the proper overlapping of the two beams (c). This capability means the fine adjustments can be made to the system while monitoring the alignment and focus through the camera's proprietary software, rather than constantly moving from table top optical component to checking adjustment effects in the microscope eyepiece. This utilization greatly reduces the alignment time and improves the alignment accuracy since the alignment can be verified with the camera's software, rather than merely visually verified via the eyepiece. Since the software has a live feed, it also solves the problem of the z-axis drift. By monitoring the live feed, the drift can instantly be recognized and compensated for by adjusting the focus knob.

\subsection{Restoring and improving resolution}

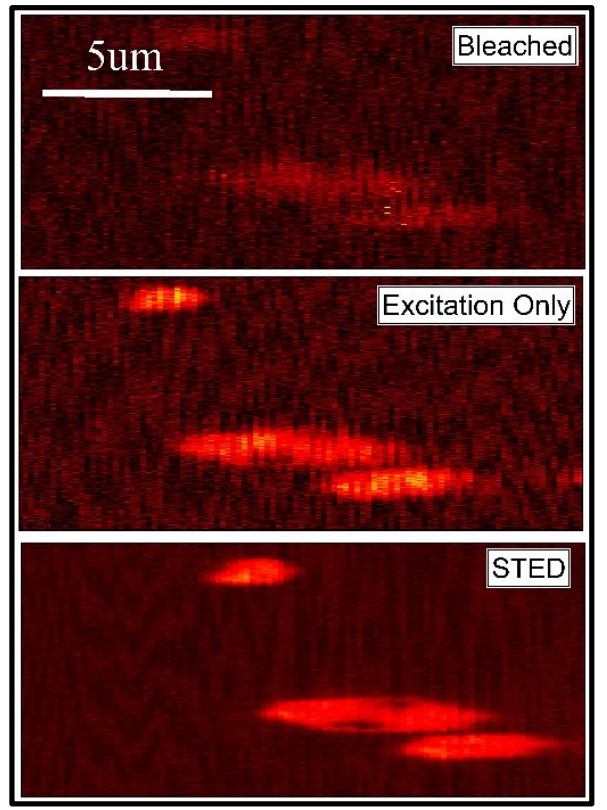

Figure 5.20: Bleached, excitation only, and CPC-STED image of GaN pits with new stage and software.

With the addition of a new stage, the system needed to be tested and recalibrated to provide useful images again. Since this project was funded through a NSF-MRI grant the result needs to be a functional system that is usable by others. In this section, I will cover 
the images we were able to bet from the system after installing the new stage. These results were aided by the improved software as well as the camera used for alignment.

Once we had the new software with the new stage, we returned to a familiar material system, the GaN pits. In Figure 5.20, we have the results of repeating the basic set of tests

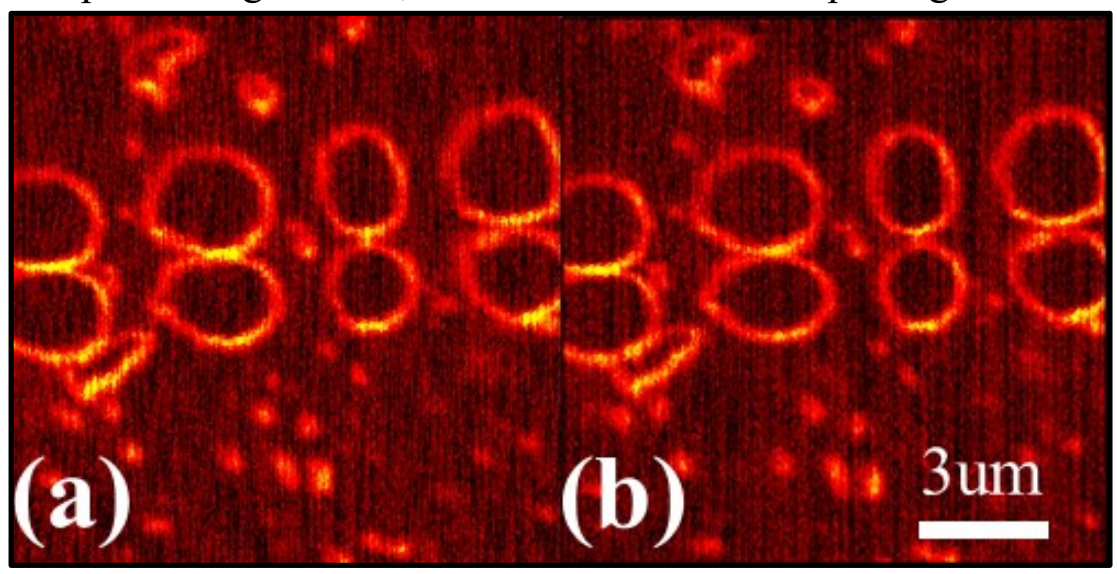

Figure 5.21: Scanning-CPC (a) and CPC-STED (b) image of resolution testing GaN pillars.

for the CPC-STED system. We see that we can still bleach an image and that the pits are still resolvable. It also shows that, at least preliminarily, CPC-STED shows an improvement in the image. With this promising result, we returned to the resolution testing pillar material system, to verify functionality once again as well as capabilities. 
This allowed for us to take advantage of a feature of the new stage, the larger scan area, as seen in Figure 5.21. We did a standard scanning-CPC (a) and a CPC-STED (b) of larger areas, $\sim 200 \mathrm{um}$, and then used only the portions that provided the best tests for the CPC-STED resolution. We can see that there is some, abet not obvious, improvement in the image from (a) to (b). This improvement can be measured directly by looking at the line scans between two pillars, separated by $\sim 200 \mathrm{~nm}$, seen in Figure 5.22. We see an obvious separation between the adjacent pillar walls when using the CPC-STED that is not apparent when using the scanning-CPC system.

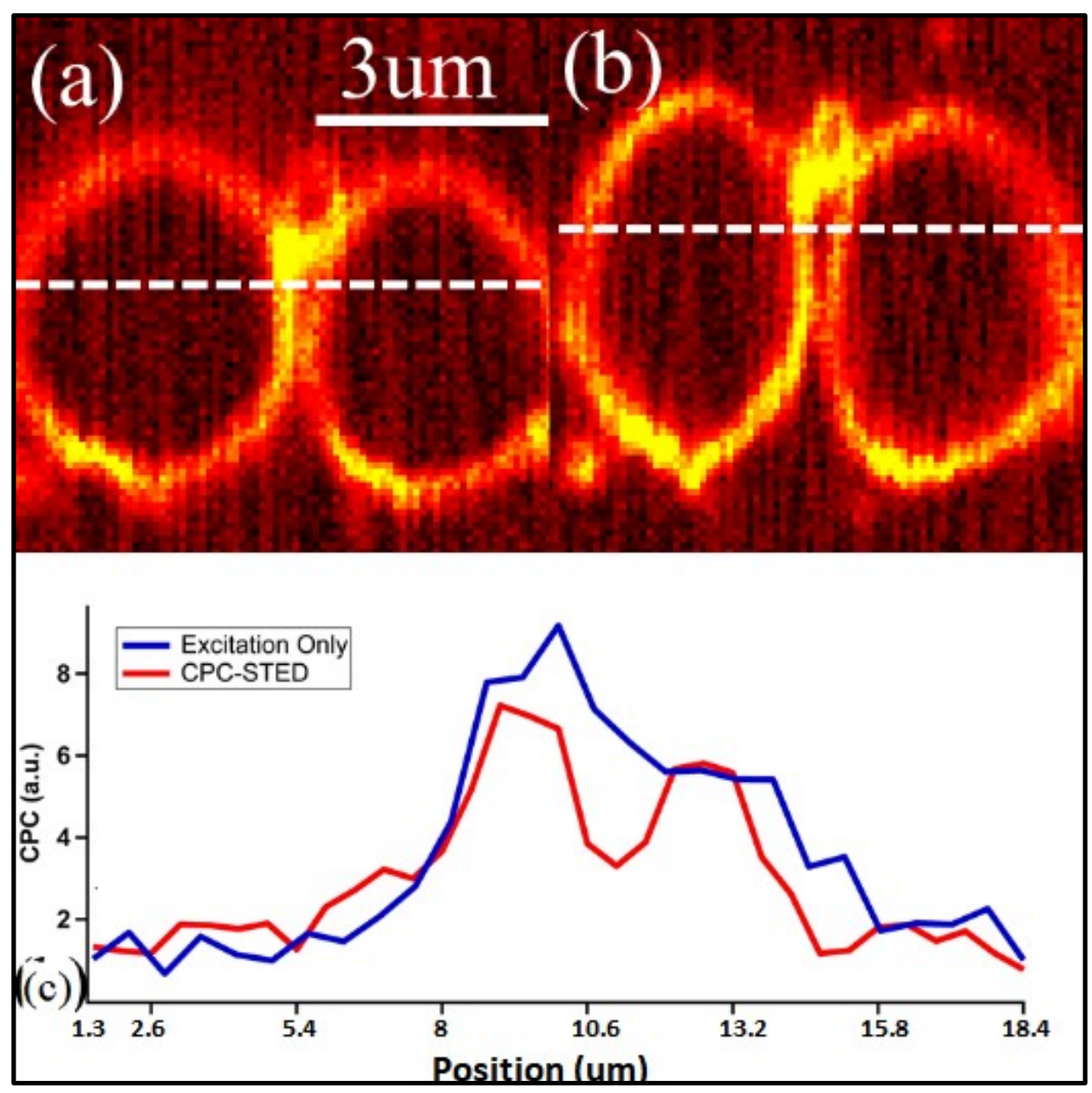

Figure 5.22: GaN pillars taken via (a) scanning-CPC and (b) $C P C$ STED, with line scans (c) of the separations. 


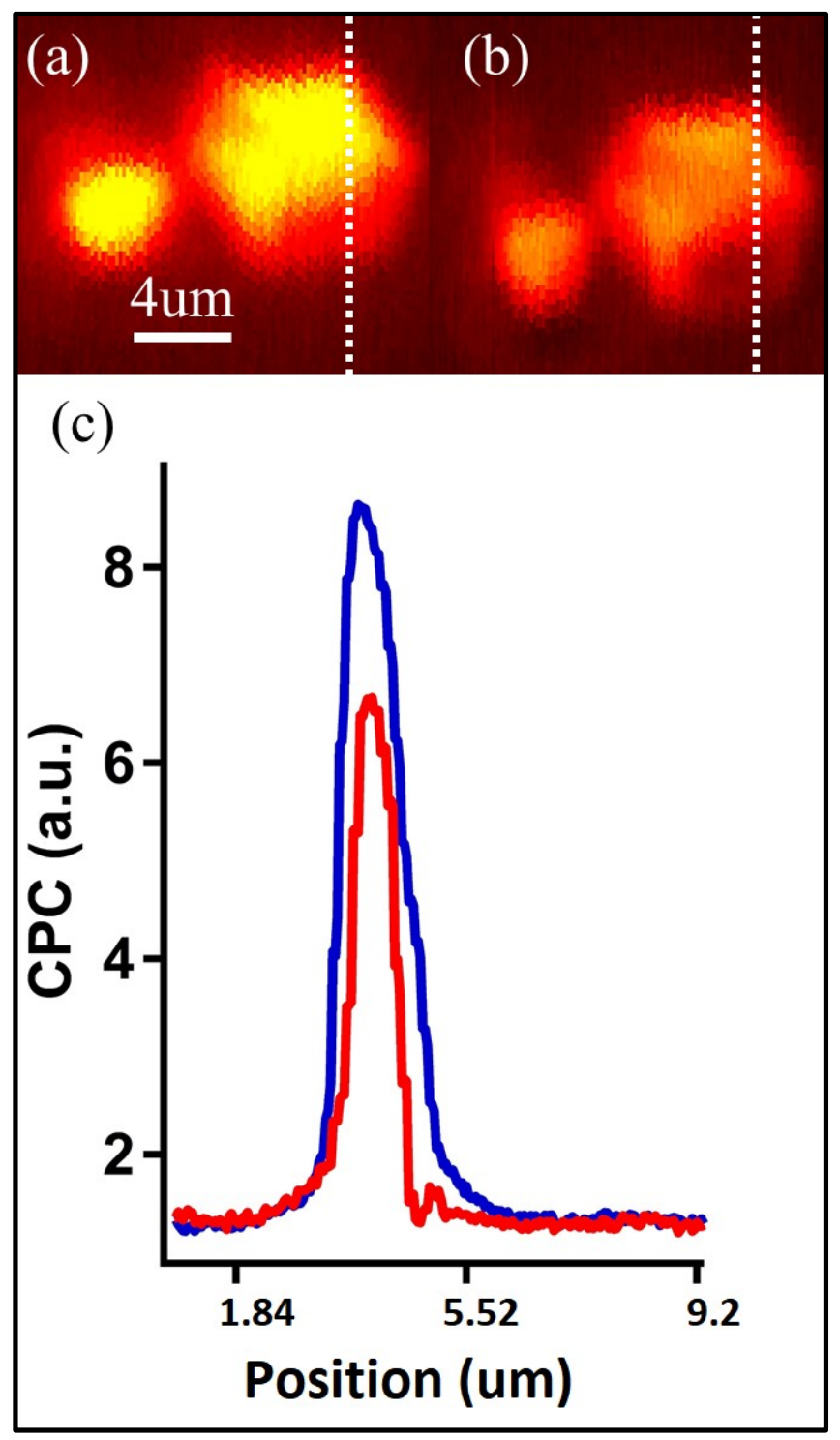

Figure 5.23: (a) scanning-CPC image, (b) CPCSTED image, and (c) line scans across both with the excitation only line trace in blue and the CPC-STED line trace in red.

Returning to the shallow GaN pits with these improvements revealed an additional interesting feature. When looking at a shallow GaN pit, we observed asymmetries, but now we are able to resolve features in the asymmetries that were previously obscured. In Figure 5.23, we have the scanning-CPC images (a) compared to a CPC-STED image (b). In Figure 5.23 (c), we have the line scans from the images taken along their respective dotted lines. The line scans also show that the blue trace using the excitation beam only is 
wider and relatively featureless along the right side, unlike the CPC-STED line trace, shown in red. The red trace also shows a secondary feature at position $\sim 190$ that is not seen in the blue trace. This feature is likely a sloped wall of the pit showing that we could differentiate the two slopes that were previously undistinguishable as a feature that is $\sim 200 \mathrm{~nm}$ wide and below the diffraction limit of the current system. This feature is further proof of resolution improvement and possible super resolution.

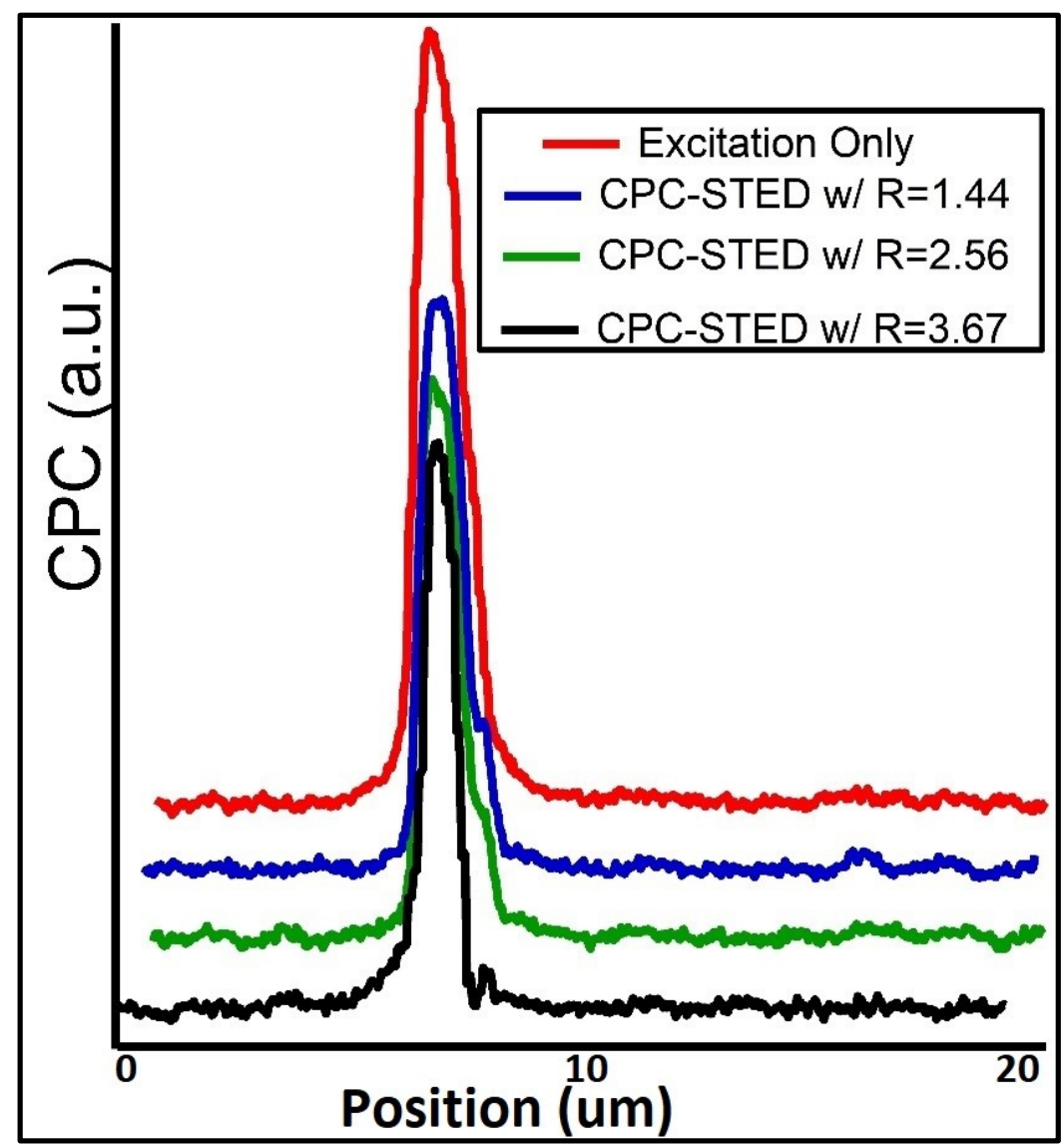

Figure 5.24: Line scans of the GaN pit seen in Figure 5.23 with varying values for $R$.

This result also provides us with the opportunity to test how varying values for $\mathrm{R}$ change the resulting resolution of a scan. In Figure 5.24, we have a series of line scans across the same GaN pit used in Figure 5.23. We have a line scan collected using only the $2.54 \mathrm{eV}$ excitation beam (red trace), CPC-STED with an $\mathrm{R}=1.44$ value (blue trace), $\mathrm{R}=2.56$ (green trace), and finally $\mathrm{R}=3.67$ (black trace). These traces are offset along the 
y-axis to provide room to see the secondary feature, also seen in Figure 5.23 (c) at position $\sim 190$. This finding shows that as the $\mathrm{R}$ value increases, the asymmetry of the pit also increases until a maximum value is reached, $\mathrm{R}=3.67$, and the second slope of the GaN pit resolves itself from the primary feature. Resulting data showed resolution improvement and experimental evidence that the mechanism described by Dr. Stefan Hell can be applied to scanning-CPC to create a new, improved-resolution scanning technique: CPC-STED.

This chapter has shown that the principles discovered in fluorescent STED microscopy can be applied to a scanning-CPC system. This application of principles provides the framework for a new scanning system with possible super resolution. This chapter also demonstrates that the CPC-STED system, even using the new stage with its poorer resolution, remains capable of producing better images when utilizing the CPCSTED methodology rather than the scanning-CPC method. 


\section{CHAPTER 6 CONCLUSION AND FURTHER WORK}

This dissertation has shown that within the body of work describing the advantages and unique information provided by capacitive photocurrent (CPC) spectroscopy there is a place for a scanning-CPC system. This system is easy to add to an existing Stimulated Emission Depletion (STED) without hindering the functionality of a STED system. This work also provides the software backbone for efficient scanning and the schematics for the optical layout for materials that have absorbance or electrical responses at $2.09 \mathrm{eV}$ and $2.54 \mathrm{eV}$.

Also, we have shown that this CPC-STED technique provides a deterministic microcopy method for achieving subdiffraction images. We have also shown some of the limitations of this technique: waveguiding effect, sample cleanliness or preparation

method, and experiment design. With these developments, there is also a wide range of work that can be done in the future.

\subsection{Future work}

Here we will discuss potential future avenues of scientific inquiry. Many of these were not accomplished in this dissertation due to funding limitations.

\subsubsection{Hardware/Software improvements}

\subsubsection{Objective}

A major limitation of the CPC-STED system is the objective used. Since it has such a low numerical aperture $(\mathrm{NA}=0.8)$, the minimum spot size is significantly larger 
than if we used an objective with a larger numerical aperture. We can see in Figure 6.1 that with a better objective the resolution can be increased dramatically, from $171 \mathrm{~nm}$ to $\sim 100 \mathrm{~nm}$. This course was not pursued because the high NA objectives can be upward of $\sim \$ 5,000$.

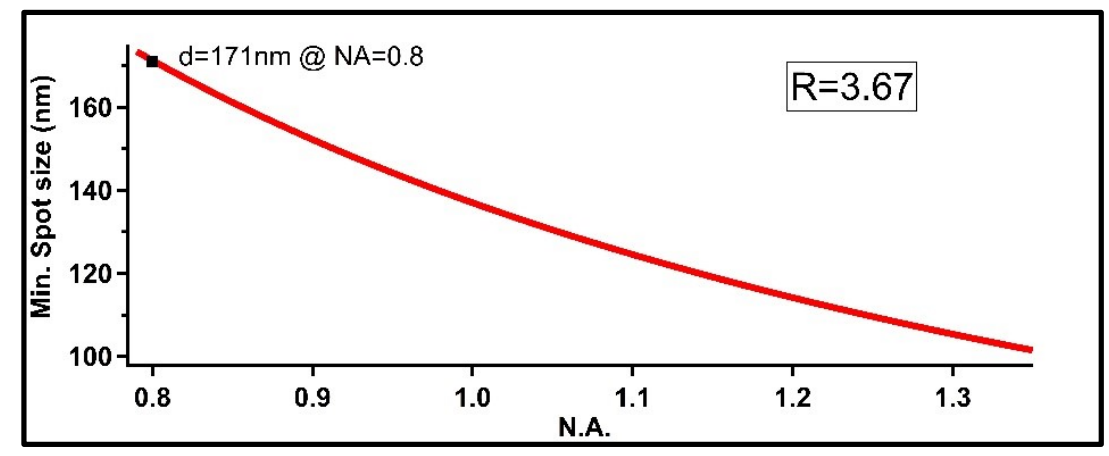

Figure 6.1: Effect of numerical aperture on theoretical minimum spot size with a $2.09 \mathrm{eV} / 2.54 \mathrm{eV}$ system, using a $R=3.67$ value.

\subsubsection{Stage and software}

With the failure of the MadCityLabs stage with its angstrom resolution, the Prior stage with its $40 \mathrm{~nm}$ minimum step size is significantly worse for a scanning system. The manufacturer can improve the step size of the Prior Scientific stage to $\sim 20 \mathrm{~nm}$ for $\sim \$ 3000$ or the MadCityLabs stage could be returned and go through the RMA repair process for $\sim \$ 10000$. Both option were beyond the funding available to this project. If a new stage is purchased, then it can be one with three-axis of motion, rather than only two. This improvement means that data could be collected on various levels of a sample probing various levels and their different electronic responses.

Since the CPC-STED system is built around and does not impede the STED system, it would be advantageous to use both simultaneously. With a few hardware upgrades, a CPC image can be built simultaneously with a florescence image or a simple 
back reflection image. This addition will help to better correlation topography with CPCSTED images, ensuring that features are accurately quantified and qualified, rather than relying only on photographs taken before or after a scan.

\subsubsection{Light source}

Another component of the system that can be adjusted is the light source. Unlike STED microscopy where specific fluorophores can be added to a sample and probed, in CPC-STED the sample is directly probed, which means that the light source needs to be tuned to probe different states of a sample. Some options would be to have the excitation beam use the Continuum or OPA system as its light source. With these improvements in place, a spectrum of scans could be compiled to possibly reveal more information on a samples electronic structure. The problem with this is both the system uses a single-mode fiber (SMF) to help with the collimation and a SMF is not suitable to have such a broad spectrum of beams.

\subsubsection{Material systems}

A final avenue for investigation is considering more material systems. We investigated $\mathrm{Si}, \mathrm{GaN}, \mathrm{PCBM}, \mathrm{GaAs}$, and perovskites material systems. With the development of this technique, we looked at systems we have studies previous or systems that other member of our group were studying such that the information CPC-STED provided would be helpful. There are multiple systems that can be studied and should be investigated using this new scanning technique. 


\section{REFERENCES}

1. Shuvra, P.D., et al., Axial asymmetry for improved sensitivity in MEMS piezoresistors. Journal of Micromechanics and Microengineering, 2016. 26(9): p. 11.

2. Shah, H., et al., Optical Generation and Detection of Polaronic States in PCBM. Journal of Physical Chemistry C, 2013. 117(50): p. 26538-26542.

3. Fianium, FemtoPower 1060: Supercontinuum Laser Source. PT-D00020, Fianium: Southhampton, UK.

4. Spectra-Physics, OPA-800C: Ultrafast Optical Parametric Amplifier. 2002, Spectra-Physics: Mountain View, CA. p. 122.

5. Spectra-Physics, Hurricane: Integrated Ti:Sapphire Regenerative Amplifier System. 2004, Spectra-Physics: Mountain View, CA. p. 90.

6. Utah, U.o. Super-Resolution Microscopy Tutorial. 2011 [cited 2017 Novermber, 17]; Available from: http://advanced-microscopy.utah.edu/education/super-res/.

7. Swapp, S. Scanning Electron Microscopy (SEM). [Online] 2017 [cited 2017 Oct 10]; Available from:

https://serc.carleton.edu/research education/geochemsheets/techniques/SEM.html 
8. Carver, A.L., et al. Photoinduced charge transfer across an organic/contact interface: polaronic state spectroscopy. in SPIE NanoScience + Engineering. 2013. SPIE.

9. Shin, D.S., Systematic analysis of the photocurrent spectroscopy on InGaN/GaN blue light-emitting diodes. IEEE Journal of Quantum Electronics, 2013. 49(12): p. 1062.

10. Casey Jr., H.C., D.D. Sell, and K.W. Wecht, Concentration dependence of the absorption coefficient for $n$ - and p-type GaAs between 1.3 and $1.6 \mathrm{eV}$. Journal of Applied Physics, 1975. 46(1): p. 250-257.

11. Ioannou, D.E. and C.A. Dimitriadis, A SEM-EBIC minority-carrier diffusionlength measurement technique. IEEE Transactions on Electron Devices, 1982. 29(3): p. 445-450.

12. Sharma, A. and S.G. Schulman, Introduction to fluorescence spectroscopy. Techniques in analytical chemistry series; Techniques in analytical chemistry series. 1999, New York :: Wiley.

13. Sarid, D., Scanning force microscopy : with applications to electric, magnetic, and atomic forces. Oxford series on optical sciences ; 2; Oxford series in optical and imaging sciences ; 2. 1991, New York :: Oxford University Press.

14. Bansal, T., et al., New insights into the density of states of graphene oxide using capacitive photocurrent spectroscopy. Carbon, 2012. 50(3): p. 808-814. 
15. Fianium, AOTF: User Manual. PX-00027(1), Fianium: Southhampton, UK.

16. Tu, R. Ray Optics Simulation. 2017; Available from:

https://ricktu288.github.io/ray-optics/.

17. Mallajosyula, A.T., et al., Large-area hysteresis-free perovskite solar cells via temperature controlled doctor blading under ambient environment. Applied Materials Today, 2016. 3: p. 96-102.

18. Saleh, B.E.A. and M.C. Teich, Photons and Atoms, in Fundamentals of Photonics. 2001, John Wiley \& Sons, Inc. p. 423-459.

19. Hess, S.T., T.P.K. Girirajan, and M.D. Mason, Ultra-high resolution imaging by fluorescence photoactivation localization microscopy. Biophysical Journal, 2006. 91(11): p. 4258-4272.

20. Abeyweera, B.K. and B.W. Alphenaar, Thickness dependent red shift of the photocurrent spectrum in bulk heterojunction solar cells. Applied Physics Letters, 2013. 102(4): p. 3.

21. Mohite, A., et al., Displacement current detection of photoconduction in carbon nanotubes. Applied Physics Letters, 2005. 86(6): p. 3.

22. Tsai, H., et al., High-efficiency two-dimensional Ruddlesden-Popper perovskite solar cells. Nature, 2016. 536(7616): p. 312-316. 
23. Ambacher, O., et al., Sub-bandgap absorption of gallium nitride determined by photothermal deflection spectroscopy. Solid State Communications, 1996. 97(5): p. $365-370$.

24. Chen, C.Y., et al., Electron beam induced current in InSb-InAs nanowire type-III heterostructures. Applied Physics Letters, 2012. 101(6): p. 3.

25. Lakowicz, J.R., Principles of fluorescence spectroscopy. 3. ed., [4. corr. print.] ed. 2010, New York :: Springer.

26. Pankove, J.I., Optical processes in semiconductors. Solid state physical electronics series; Solid state physical electronics series. 1971, Englewood Cliffs, N.J.: Prentice-Hall.

27. Tinnefeld, P., et al., Far-Field Optical Nanoscopy. Softcover reprint of the original 1st ed. 2015 ed. Springer Series on Fluorescence 14. 2016, Berlin: Springer Berlin.

28. Mohite, A., et al., Photocurrent spectroscopy of double wall carbon nanotubes. Chemical Physics Letters, 2005. 412(1-3): p. 190-194.

29. Mohite, A., et al., Field-enhanced photocurrent spectroscopy of excitonic states in single-wall carbon nanotubes. Nano Letters, 2006. 6(7): p. 1369-1373.

30. Mohite, A.D., et al., Exciton dissociation and stark effect in the carbon nanotube photocurrent spectrum. Nano Letters, 2008. 8(1): p. 142-146. 
31. Gopinath, P., et al., Strain tuning of the photocurrent spectrum in single-wall carbon nanotubes. Nano Letters, 2007. 7(10): p. 3092-3096.

32. Nie, W., et al., High-efficiency solution-processed perovskite solar cells with millimeter-scale grains. Science, 2015. 347(6221): p. 522-525.

33. Niu, G.D., X.D. Guo, and L.D. Wang, Review of recent progress in chemical stability of perovskite solar cells. Journal of Materials Chemistry A, 2015. 3(17): p. $8970-8980$.

34. Matys, M. and B. Adamowicz, Mechanism of yellow luminescence in GaN at room temperature. Journal of Applied Physics, 2017. 121(6): p. 065104.

35. Degraeve, R., et al., New insights in the relation between electron trap generation and the statistical properties of oxide breakdown. IEEE Transactions on Electron Devices, 1998. 45(4): p. 904-911.

36. Zaidi, Z.H., et al., Sulfuric acid and hydrogen peroxide surface passivation effects on AlGaN/GaN high electron mobility transistors. Journal of Applied Physics, 2014. 116(24): p. 244501.

37. OlympusAmerica, Microscope Components Guide: Choosing The Ideal UIS2 Optics Components For Your Equipment. 2008.

38. Hell, S.W., Far-Field Optical Nanoscopy. Science, 2007. 316(5828): p. 11531158. 


\section{APPENDIX A}

Capacitive Photocurrent - Stimulated Emission Depletion (CPC-STED) Standard Operating procedure
I. Assumed Parameters
II. Aligning 488nm
III. Aligning 529nm
IV. Finalizations
V. Common Issues and Solutions

I. Assuming the following parameters:

- $488 \mathrm{~nm}$ is will aligned through PS, FM2, and FM4

- $\quad 488 \mathrm{~nm}$ is well collimated after C2

- Using 592nm splitters after FM3

II. Aligning 488nm

- At FM4 without C2 ensure the coupling ins good, ie. The output is Gaussian

1) Gaussian Beam Example

i. Using two (2) irises with heights centered at the cross of the microscope's back cover, check the height and centering from FM4 (still without C2). 
a. Proper height is the focus of this step.

b. Add C2

c. Adjust C2 to match the height of FM4

d. Ensure maximum power is in the center of the collimated beam (true Gaussian), not shifter away from center. This can be a visual check.

e. Check beam centering on both irises

ii. TIP: With good beam uniformity / shape adjust C2 pan/tilt not XYZ.

iii. Permanently affix irises to help with next steps and future alignments and adjustments.

iv. Double check collimation after C2 (looking for parallel lines, using the small plate).

2) Well collimated example

i. Align with DM3 to and through the microscope

a. Checking the height at the microscope

b. Looking at height with the microscope front off

c. Looking through oculars

d. At DM3 adjust X, PAN, and TILT in opposite motions to correct aberrations

e. The back reflected 488 should be along the same, incoming beam path. 
ii. $488 \mathrm{~nm}$ back reflection should be on the $592 \mathrm{~nm}$ depletion beam path

3) Aligning $592 \mathrm{~nm}$

i. Same steps as $488 \mathrm{~nm}$, checking for collimation and height. ii. Using DM4 to align beams together.

\section{Finalizations}

- With the front of the microscope off a rough alignment can be check by looking at the overlay of $488 \mathrm{~nm}$ and $592 \mathrm{~nm}$ on the far wall.

1) Focus adjustments on DM4

2) Now the phase plate can be added $(592 \mathrm{~nm}$ is the center of the 2nd row)

i. Check pan/tilt to insure back reflection is still good

For the fine alignment two (2) mirrors and irises (with height set to match other irises) one right in front of microscope back and across table. This checks height, collinearity, centering, and overlap of $488 \mathrm{~nm}$ and $592 \mathrm{~nm}$. 


\section{CURRICULUM VITA}

$\begin{array}{ll}\text { NAME: } & \text { Austin Levi Carver } \\ \text { ADRESS: } & 1047 \text { Oakwood Ave. } \\ & \text { Louisville, KY } 40215 \\ \text { DOB: } & \text { May 23, } 1990 \\ \text { EDUCATION: } & \text { B.S., Physics } \\ & \text { University of Louisville } \\ & \text { 2008-2012 } \\ & \text { M.S., Physics } \\ & \text { University of Louisville } \\ & \text { 2012-2013 } \\ & \text { Ph.D., Electrical Engineering } \\ & \text { University of Louisville } \\ & \text { 2013-2017 }\end{array}$

AWARDS:

University of Louisville, Graduate Fellowship.

University of Louisville, J.B. Speed School of Engineering, Theobald Scholarship Award. 


\section{PROFESSIONAL SOCIETIES:}

Sigma Pi Sigma

\section{PUBLICATIONS:}

Carver, A.L., Fernando, K., Shah, H.M., Kolli, S., Abeyweera, B., Alphenaar, B.W., Lisenkov, S. (2013). Photoinduced charge transfer across an organic/contact interface: polaronic state spectroscopy. Paper presented at the SPIE NanoScience + Engineering.

Shah, H., Carver, A.L., Fernando, K., Kolli, S., Abeyweera, B., Lisenkov, S., Alphenaar, B.W. (2013). Optical Generation and Detection of Polaronic States in PCBM. Journal of Physical Chemistry C, 117(50), 26538-26542.

doi:10.1021/jp4111986 